\title{
Quantitative brain MRI at 7T in healthy subjects and in metabolic diseases
}

Citation for published version (APA):

Haast, R. A. M. (2018). Quantitative brain MRI at $7 T$ in healthy subjects and in metabolic diseases.

[Doctoral Thesis, Maastricht University]. Maastricht University. https://doi.org/10.26481/dis.20180627rh

Document status and date:

Published: 01/01/2018

DOI:

10.26481/dis.20180627rh

Document Version:

Publisher's PDF, also known as Version of record

\section{Please check the document version of this publication:}

- A submitted manuscript is the version of the article upon submission and before peer-review. There can be important differences between the submitted version and the official published version of record.

People interested in the research are advised to contact the author for the final version of the publication, or visit the DOI to the publisher's website.

- The final author version and the galley proof are versions of the publication after peer review.

- The final published version features the final layout of the paper including the volume, issue and page numbers.

Link to publication

\footnotetext{
General rights rights.

- You may freely distribute the URL identifying the publication in the public portal. please follow below link for the End User Agreement:

www.umlib.nl/taverne-license

Take down policy

If you believe that this document breaches copyright please contact us at:

repository@maastrichtuniversity.nl

providing details and we will investigate your claim.
}

Copyright and moral rights for the publications made accessible in the public portal are retained by the authors and/or other copyright owners and it is a condition of accessing publications that users recognise and abide by the legal requirements associated with these

- Users may download and print one copy of any publication from the public portal for the purpose of private study or research.

- You may not further distribute the material or use it for any profit-making activity or commercial gain

If the publication is distributed under the terms of Article $25 \mathrm{fa}$ of the Dutch Copyright Act, indicated by the "Taverne" license above, 


\section{QUANTITATIVE BRAIN MRI AT TT in}

HEALTHY SUBJECTS AND IN METABOLIC DISEASES

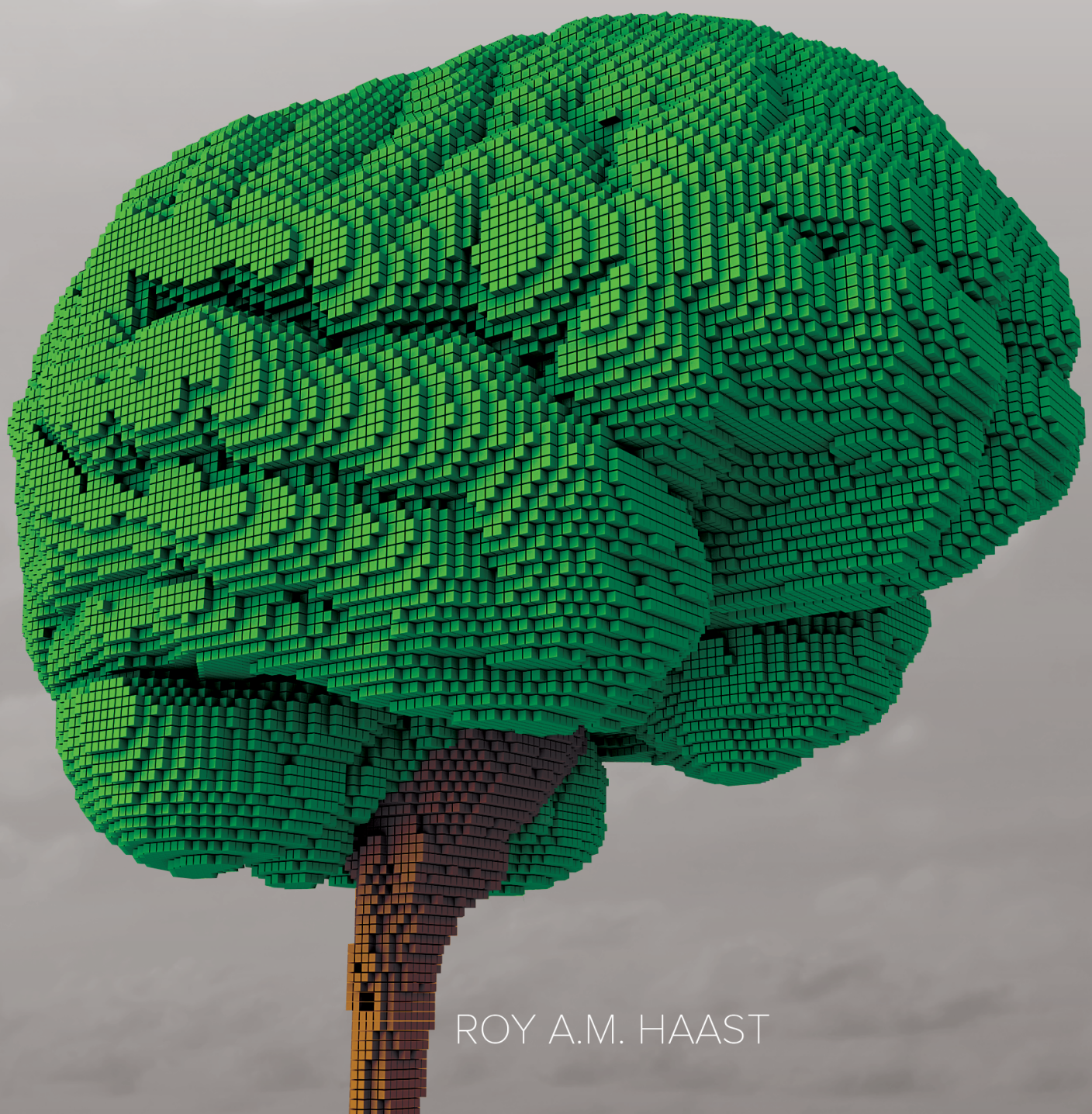


(c) Roy A.M. Haast, Maastricht 2018

All rights reserved. No part of this thesis may be reproduced, stored in a retrieval system, or transmitted in any form, or by any means, electronic, mechanical, photocopying, recording, or otherwise, without prior written permission of the author.

The work presented in this thesis was funded by the NWO Applied and Technical Sciences (grant number 12724).

\section{ISBN:}

978-94-028-1095-0

\section{Printed by:}

Ipskamp Printing B.V., Enschede 


\section{QUANTITATIVE BRAIN MRI AT 7T in}

HEALTHY SUBJECTS AND IN METABOLIC DISEASES

\section{DISSERTATION}

to obtain the degree of Doctor at Maastricht University,

on the authority of Rector Magnificus, Prof. Dr. Rianne M. Letschert,

in accordance with the decision of the Board of Deans,

to be defended in public on

Wednesday, June 27, 2018 at 10:00 hours

by

Roy Andreas Marinus Haast 


\section{Supervisor:}

Prof. Dr. Elia Formisano

\section{Co-supervisor:}

Dr. Kâmil Uludağ

Dr. Jacobus F.A Jansen

\section{Assessment Committee:}

Prof. David Linden (Chair)

Prof. David G. Norris (Donders Centre for Cognitive Neuroimaging, Nijmegen)

Dr. Itamar Ronen (Leiden University Medical Center, Leiden)

Dr. Benedikt A. Poser 


\section{CONTENTS}

$\begin{array}{lll}\text { Chapter } 1 & \text { General introduction } & 7\end{array}$

Chapter 2 The effect of $\mathrm{B}_{1}{ }^{+}$correction on MP2RAGE T 1 and 37 apparent cortical thickness at 7 Tesla

Chapter 3 Reproducibility and reliability of quantitative and weighted $\mathrm{T}_{1}$ and $\mathrm{T}_{2}{ }^{*}$ mapping for myelin-based cortical parcellation at 7 Tesla

Chapter 4 Anatomic \& metabolic brain markers of the m.3243A>G mutation: a multi-parametric 7 Tesla MRI study

Chapter 5 Imaging genetics in Type 2 Diabetes Mellitus

Chapter 6 Summary and general discussion

Addenda Knowledge valorization

Acknowledgments

Curriculum Vitae

Publications 
GENERAL INTRODUCTION 
The concurrent discoveries by Sir Peter Mansfield and Paul Lauterbur in 1972 (Lauterbur, 1973; Mansfield and Grannell, 1973) paved the way for magnetic resonance imaging (MRI) to evolve into one of the most widely used clinical tools for imaging the brain. What started with a single MRI scanner and magnetic field strength of 0.1 Tesla (T, Damadian (1974)), became a major biomedical technology with more than $40.000 \mathrm{MRI}$ scanners used worldwide for clinical routine $(\leq 3 \mathrm{~T})$ and/or research purposes $(\leq 10.5 \mathrm{~T}$, Vaughan et al. (2006); Moser et al. (2012)).

Unlike the tremendous developments in MRI hardware and methodologies, the main physical principles remained unchanged (Bloch, 1946; Purcell et al., 1946). Once inside the scanner, the ${ }^{1} \mathrm{H}$ protons or other nuclei with magnetic moments align on average with the magnetic field $\left(B_{0}\right)$ direction (e.g. usually defined as the z-direction), but continuously precess at a specific rate around the $z$-axis $\left(\omega_{0}=\gamma B_{0}\right.$, i.e. the frequency at which they are precessing, called Larmor frequency) (Larmor, 1897). Essentially, the MRI signal is produced by exciting specific atomic nuclei (e.g. hydrogen $\left({ }^{1} \mathrm{H}\right)$ in most approaches) with radiofrequency (RF) pulses inside the body and measuring the return of their (transversal or longitudinal) magnetization to its equilibrium value. By applying an RF pulse, tuned to the Larmor frequency, the magnetization is flipped away from the $B_{0}$ direction towards the $x y$-plane, i.e. perpendicular to the main magnetic field. Here, the chosen flip angle $\left(a,^{\circ}\right)$ determines how far the net magnetization vector is tilted (Ernst and Anderson, 1966). After the excitation RF pulse, the magnetization will return to the equilibrium state. That is, the magnetization in the $x y$-plane will decay to zero by dephasing or relaxation with a time constant called $\mathrm{T}_{2}{ }^{(*)}$, and the magnetization in the z-direction will grow back to its initial value by relaxation with a time constant called $\mathrm{T}_{1}$, hereby gradually releasing all energy from the RF pulse. The signal is measured at the echo time (TE, ms) after the RF excitation and this procedure will be repeated at the repetition time (TR, ms) (Bloch, 1946). Both the $\mathrm{T}_{2}{ }^{(*)}$ and $\mathrm{T}_{1}$ values depend on the biochemical composition of tissues, and each tissue type will show a distinct behavior in response to the RF pulse, allowing the internal structures of the human body, such as the brain, to be characterized by the differences in their signal intensity as a function of TE and TR (Edzes and Samulski, 1977). As such, by using optimized sequence designs and parameters, the contrast can be manipulated to highlight specific properties of the brain, including specific macromolecular compound-rich regions in anatomical MRI or changes in the concentration of the deoxygenated blood in functional MRI (Bydder and Young, 1985; Ogawa et al., 1990). To localize the origin of the signal, controlled spatiotemporal manipulations of $B_{0}$ are applied using magnetic field gradients (in the range of $\mathrm{mT}$ differences) across the $\mathrm{x}$-, $y$ - and z-planes (Lauterbur, 1989). Depending on the aim of the MR sequence design 


\section{CHAPTER 1}

(e.g. 2D, slice-by-slice, vs. 3D, volume-by-volume, acquisitions), each gradient can be used for slice selection $\left(G_{s}\right)$ or in-plane (frequency, $G_{f^{\prime}}$ and phase, $G_{p}$ ) encoding. As a result, the ${ }^{1} \mathrm{H}$ spins precess at different frequencies and phases at different locations and the superstition of all these signals from all these spins are acquired and stored into k-space. Fourier transformation of k-space then allows the reconstruction of the tissue in Cartesian coordinates in the image space (Twieg, 1983).

\section{1. | ULTRA-HIGH FIELD IMAGING}

The available magnetic field strengths for human MRI scanners rapidly evolved over the last 40 years from $\leq 1 \mathrm{~T}$ (low-mid field) to $\leq 3 \mathrm{~T}$ (high field, gold standard for clinical diagnosis nowadays) and > 3T (ultra-high field, UHF) (Plantinga et al., 2014; Ugurbil, 2017). The main advantage of higher field strength is the increased longitudinal magnetization (i.e. more protons spins that align with $\mathrm{B}_{0}$ ) leading to increased intrinsic signal-to-noise (SNR, $\sim \mathrm{B}_{0}^{1.65}$, see Fig. 1.1) and contrast-to-noise (CNR) ratios (Vaughan et al., 2001; Norris, 2003; Duyn et al., 2007; Pohmann et al., 2016). In MRI, SNR is defined as the ratio of the signal and the background noise, while CNR describes the ability to differentiate between two sources based on their signal difference with respect to the shared background noise. Increasing $B_{0}$ allows in theory, therefore, acquisition of higher resolution data than what is achievable at lower field strengths within the same imaging time and comparable SNR and/or CNR (Pfeuffer et al., 2002; Yacoub et al., 2008; Gardener et al., 2009; Stuber et al., 2014; Ivanov et al., 2017; Keuken et al., 2017; Uludag and Blinder, 2017). Theoretically, this is especially advantageous in a clinical setting, where short imaging times are crucial. In addition, parallel imaging acceleration results in lower SNR penalties at higher field strengths due to smaller spatial overlap of the receive fields of multi-channel array coils.

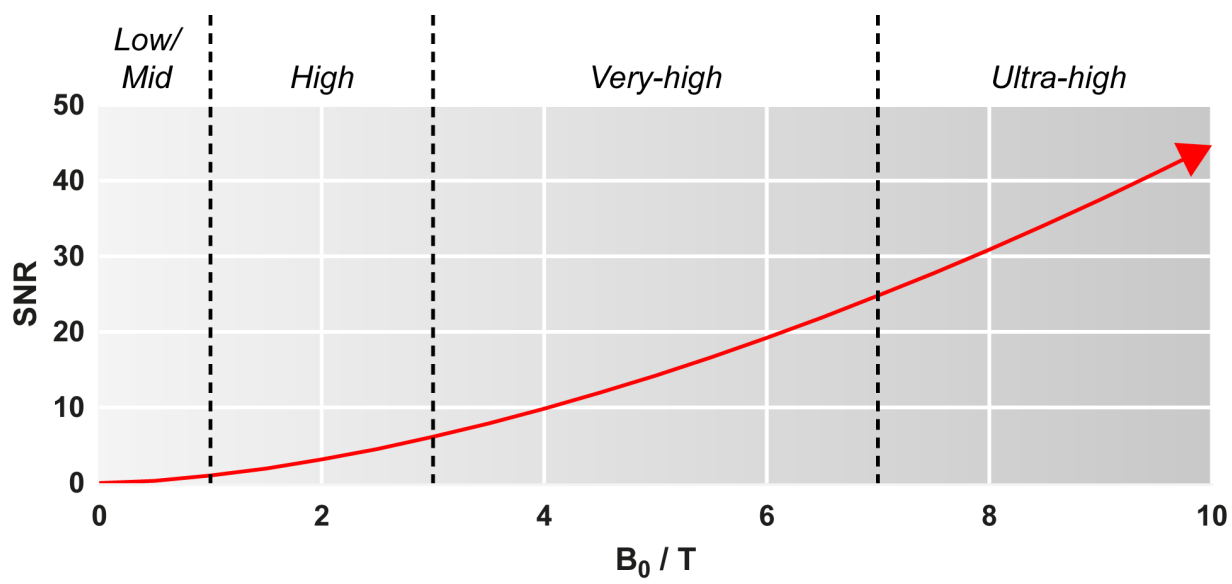

Fig. 1.1. | The red line represents fitting results on the $S N R$ as $S N R_{0}=B_{0}^{1.65}$. 
Nevertheless, the SNR and CNR benefits are (partially) counteracted by negative effects. Dizziness, nausea and metallic taste are recognized physiological side effects (Moser et al., 2012), which may affect the patient's/volunteer's comfort. In addition, on the technical side, increasing the field strength also increases the specific absorption rate $(S A R)$ and reduces the $B_{0^{\prime}} R F$ transmit $\left(B_{1}^{+}\right)$and receive $\left(B_{1}{ }^{-}\right)$homogeneities, introducing intensity biases throughout the image (Vaughan et al., 2001). These can potentially hamper diagnosis and further analyses, but there are several methods to (partially) overcome them. For example, improved shimming techniques and the use of $\mathrm{RF}$ receive arrays with tight-fitting multi-element transmit coils improve $\mathrm{B}_{0}$ and $\mathrm{B}_{1}^{+}$ homogeneity, respectively (see Ugurbil (2017) for a review on the related technical developments). Importantly, $\mathrm{B}_{1}{ }^{-}$inhomogeneities can be fully eliminated by using quantitative MR imaging (qMRl) approaches (Tofts, 2005). In contrast to the conventional clinical weighted approaches, which - although 'weighted' towards a specific tissue parameter - only provide qualitative markers on a mixture of tissue parameters, qMRI permits direct absolute comparison of images between subjects based on individual tissue characteristics. These approaches are especially useful for longitudinal (e.g. interventions or aging), cross-sectional (i.e. healthy vs. diseased) and multicenter studies.

Methods to quantitatively map, for example, gray and white matter properties (e.g. using relaxometry and diffusion MRI) or blood flow (using arterial spin labeling) are available and better accessible due to faster imaging times for UHF MRI (Ugurbil, 2017). However, the available sequences (but also hard- and analysis software) are still under active development in order to minimize the artifacts that come with the higher magnetic field strengths. The work in the present thesis explores the advantages and disadvantages of a multi-parametric qMRI brain protocol at 7T in both healthy subjects and patient populations related to metabolic diseases (i.e. m.3243A $>\mathrm{G}$ and Type 2 Diabetes Mellitus patients). The remainder of this chapter will review and highlight the topics required to provide the necessary background for the following chapters.

\subsection{Q QUANTITATIVE BRAIN IMAGING}

Ideally, for clinical diagnosis and prognosis, brain images are expected to be quantitative, reproducible (i.e. precise), indicative of tissue parameters and independent of imaging sites or scanner vendors. Instead, brain MRI is typically used in a qualitative fashion, with the requirement that a trained neuro-radiologist interprets the relative values (i.e. with respect to a control/reference region) to define hyper- or hypo-intense structures using a MRI sequence weighted towards $T_{1}, T_{2}{ }^{*}$, proton density (PD) or combination of these MRI parameters. Typically, clinical, anatomical MRI sequences 


\section{CHAPTER 1}

used are $T_{1}$-weighted ( $T_{1} w$, short $T E$ and $\left.T R\right), T_{2}$-weighted $\left(T_{2} w\right.$, longer TE and $T R$ ), Fluid Attenuated Inversion Recovery (FLAIR, very long TE and TR), standard IR or diffusion-weighted imaging ( $D W I$, collection of $\mathrm{T}_{2} \mathrm{w}$ images) (see for example Bydder and Young (1985)). For most of the clinically-used scans, quantitative counterparts are available that provide (almost) pure and absolute measurements of, for example, tissue $T_{1}, T_{2}{ }^{(*)}$ and diffusion parameters (Tofts, 2005). However, they are often not used in a clinical environment, as they might require longer scanning times or advanced data analysis to calculate the quantitative values from raw images and remove biases.

\subsection{1. $\mid M R I$ relaxometry $\left(T_{1}\right.$ and $\left.T_{2}{ }^{(*)}\right)$}

As already briefly indicated above, the ${ }^{1} \mathrm{H}$ magnetization returns to its equilibrium state once flipped away from the longitudinal plane after experiencing an RF pulse. As such, one spatial unit of a MRI image (i.e. voxel) can be characterized by a) the PD (i.e. maximum longitudinal magnetization, b) the net longitudinal (expressed as $T_{1}$ in $m s$ ) and c) (effective) transversal $\left(\mathrm{T}_{2}{ }^{*}, \mathrm{~ms}\right)$ relaxation times of the total tissue composition within the voxel. The exponential longitudinal recovery (i.e. in $\mathrm{M}_{\mathrm{z}}$ direction) and transversal decay (across $M_{x y}$ ) are expressed relative to the $B_{0}$ direction and can be described using the Bloch equation (Bloch, 1946):

$$
\frac{d M(t)}{d t}=\gamma M(t) \times B(t)\left(-\frac{M_{x}+M_{y}}{T_{2}}-\frac{M_{z}-M_{0}}{T_{1}}\right)
$$

Longitudinal relaxation $-T_{1}$

$T_{1}$, which is the time needed for the exponential recovery of the net $M_{z}$ to $63 \%$ of its equilibrium value, is determined by the mobility of (predominantly) water molecules and, hence, restricted mobility due to binding to macromolecules. More specifically, $T_{1}$ is determined by the energy spectrum of the motion of proton-containing molecules, i.e. protons with excited spins release energy at the resonance frequency to its environment via mechanical collision. As the energy spectrum of thermal motion follows a typical $1 / f$-curve and the Larmor frequency increases with field strength, the probability of energy exchange decreases with magnetic field strength, leading to an increase in $T_{1}$. Due to the different biochemical composition, water binding and PD between gray (GM) and white matter (WM) brain tissue and cerebral spinal fluid (CSF), each compartment will be characterized by different $T_{1}$ values, see Table 1.1. As such, due to the higher concentration of myelin, $T_{1}$ of WM is lower compared to GM, whereas CSF is characterized by the highest $\mathrm{T}_{1}$ due to the higher mobility of water (Koenig et al., 1990). As argued above, in addition to the biochemical composition of the tissue, $T_{1}$ also depends on $B_{0}$ (see Table 1.1) (Koenig and Brown, 1987). In most brain tissues types, $T_{1}$ increases with increasing field strength (Rooney et al., 2007). 
Table. 1.1 | Gray (GM), white matter (WM), cerebral spinal fluid (CSF) and blood T, values (in ms) obtained using 1.5T and 7T MRI. Data from Rooney, Johnson et al. (2007).

\begin{tabular}{l|l|l}
\multicolumn{2}{l}{$\mathbf{1 . 5 T}$} & $\mathbf{7 T}$ \\
\hline GM & $1188( \pm 69)$ & $132( \pm 103)$ \\
\hline WM & $656( \pm 16)$ & $1220( \pm 36)$ \\
\hline BSF & $4070( \pm 65)$ & $4425( \pm 137)$ \\
\hline
\end{tabular}

There are different approaches to quantitatively map $T_{1}$, each one being more suitable for specific study aims. All involve acquisition of data at multiple times after excitation by varying specific sequence parameters and fitting the resulting data using a non-linear least square algorithm to a model. The gold standard is the inversion recovery (IR) method, preferably combined with a rapid imaging technique, such as echo-planar imaging (EPI) for in vivo imaging, to decrease the total imaging time (see Fig. 1.2A, left) (Gowland and Mansfield, 1993). In a typical IR experiment, a $180^{\circ}$ inversion pulse is applied after which, at the inversion time ( $\mathrm{TI})$, a $90^{\circ}$ pulse flips the magnetization into the transverse plane, generating a free-induction-decay (FID). The amplitude of this FID is determined by how much the magnetization recovered during the period TI. This is repeated several times with different Tls to be able to fit the following expression to the data:

$$
S(T I)=S_{0}\left(1-2 e^{-\frac{T I}{T_{1}}}\right)
$$

$\mathrm{S}(\mathrm{TI})$ is the signal measured at time $\mathrm{TI}$ and $\mathrm{S}_{0}$ the signal that would be acquired from the equilibrium longitudinal magnetization. However, in order to reach sufficient image SNR, this experiment has to be repeated many times resulting in an imaging time that often becomes clinically impractical (Zhang et al., 1998). In addition, fast imaging techniques, such as EPI, yield unwanted geometrical distortions (see Fig. 1.2B). This hampers morphometric analyses in anatomical studies, but may be beneficial in combination with functional data to match distortions (Kashyap et al., 2017). Related to the IR approach, the Look-Locker variation utilizes an $180^{\circ}$ inversion pulse, but applies multiple low flip angle pulses spread across the TR and, thus, only requires one recovery of the longitudinal relaxation (Look and Locker, 1970). However, because of the multiple low flip angle readout pulses, it is relatively sensitive to RF pulse errors and, therefore, $\mathrm{B}_{1}^{+}$inhomogeneities. 
A

A IR-EPI

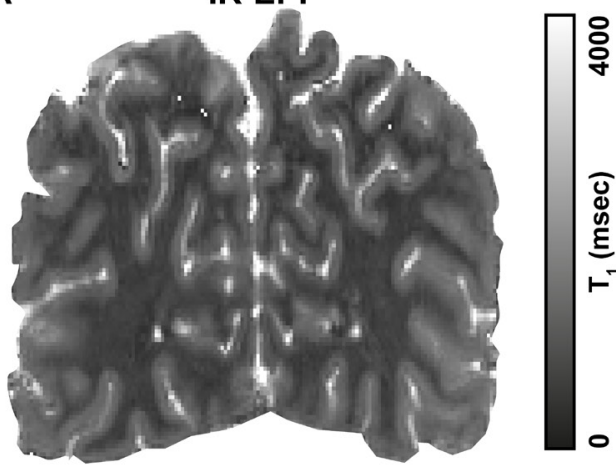

MP2RAGE

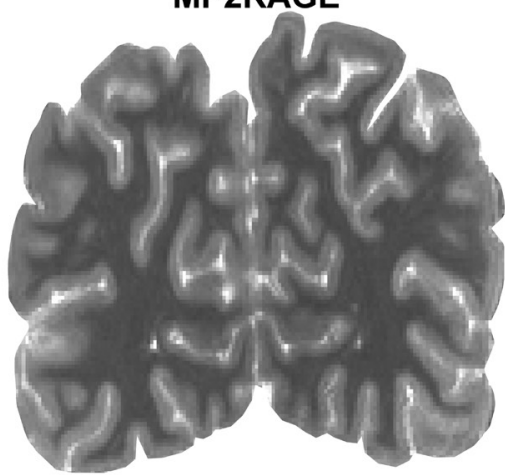

B

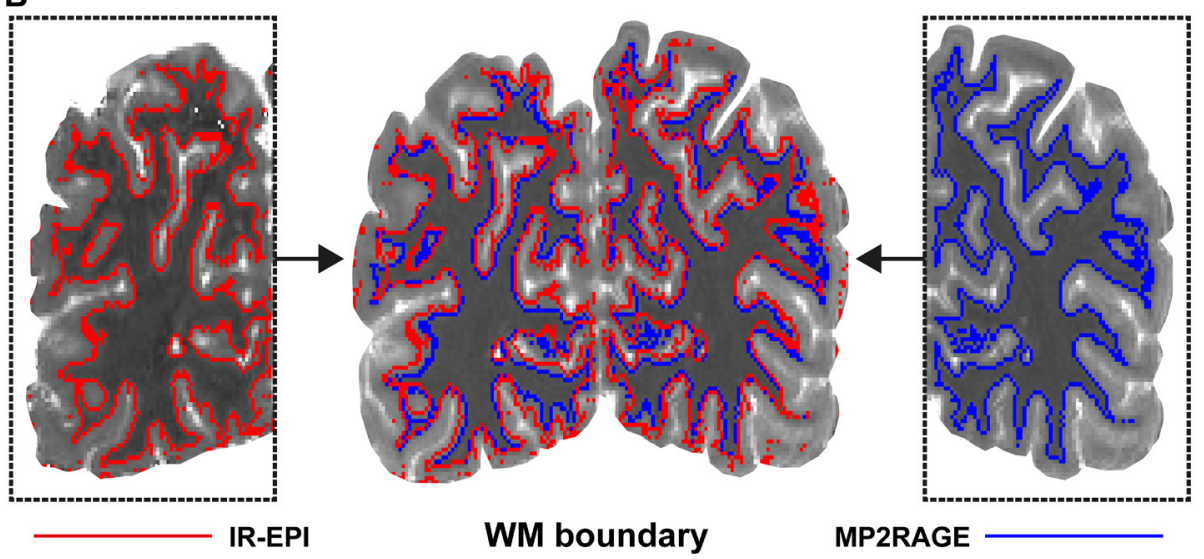

C

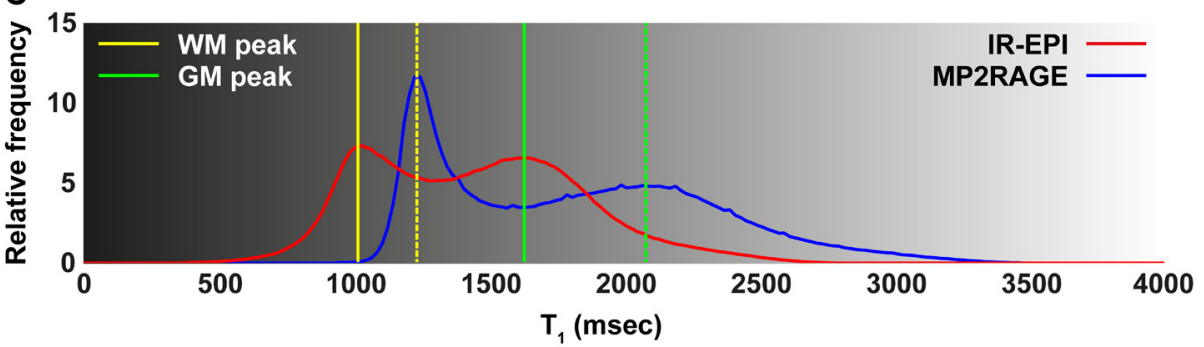

Fig. 1.2. Comparison of $T$, maps obtained using the IR-EPI (A, left) or MP2RAGE (right) approaches and corresponding white matter delineation in red and blue, repectively $(B)$. The histogram in $C$ shows the $T_{1}$ distribution for a single subject using both methods. Vertical lines indicate the average WM (yellow) and GM (green) peaks for the IR-EPI and MP2RAGE methods (solid and dashed lines, respectively)

Alternatively, $T_{1}$ can be obtained by acquiring just two images and taking the ratio of these images to estimate $T_{1}$ using a lookup table. Following this approach, wholebrain images with submillimeter resolution can be acquired within a significantly lower timeframe compared to the IR(-related) approaches. Herein, the MP2RAGE 
(Magnetization Prepared 2 Rapid Acquisition Gradient Echoes) sequence has gained a lot of popularity in the recent years, especially at higher field strengths (see Fig. 1.2A, right) (Marques et al., 2010). It was originally developed as an extension of the MPRAGE (Magnetization Prepared Rapid Acquisition Gradient Echoes) sequence (Mugler and Brookeman, 1990) to improve tissue segmentation. Whereas the MPRAGE $T_{1} w$ images are influenced by $\mathrm{M}_{0}, \mathrm{~B}_{1}{ }^{-}$and $\mathrm{T}_{2}{ }^{*}$, the MP2RAGE approach removes these effects by taking the combination of two images acquired at different TIs (Van de Moortele et al., 2009). In addition, by using different flip angles for the two gradient-echo (GRE) readout blocks at each $\mathrm{Tl}$, first order $\mathrm{B}_{1}^{+}$inhomogeneities are minimized, although not entirely, rendering the acquired MP2RAGE image (almost) purely $\mathrm{T}_{1}$-weighted. To achieve this, the MP2RAGE image is computed online by the scanner (i.e. during the scan session) as follows:

$$
M P 2 R A G E=\frac{G R E_{T I 1} G R E_{T I 2}}{G R E_{T I 1}^{2}+G R E_{T I 2}^{2}}
$$

Here, the two volumes acquired at different TIs are presented as GRE $\mathrm{T}_{11}$ and $\mathrm{GRE}_{\mathrm{T} 12}$. The final $T_{1}$ map is then calculated online with the use of a lookup table based on the sequence parameters, which were optimized to achieve maximal GM-WM and CSF-GM CNR per unit time and with minimized $\mathrm{B}_{1}{ }^{+}$-related inhomogeneities. The GM and $W M T_{1}$ values obtained using the MP2RAGE sequence corresponded well with those found in literature and were highly reproducible across subjects and within the same subject using varying sequence parameters (Marques et al., 2010). Depending on sequence parameters and resulting $\mathrm{B}_{1}{ }^{+}$dependency, the values may deviate from those with the IR-EPI approach (see Fig. 1.2C). Recently, a combined MP2RAGE and $\mathrm{B}_{1}^{+}$-mapping routine was developed to overcome the residual $\mathrm{B}_{1}^{+}$dependence of the MP2RAGE T 1 maps (Marques and Gruetter, 2013). It requires the acquisition of an additional Sa2RAGE (Saturation-prepared with 2 rapid Gradient Echoes) $\mathrm{B}_{1}{ }^{+}$map (Eggenschwiler et al., 2012) that is used to refine the $T_{1}$ values post-hoc based on the actual $\mathrm{B}_{1}{ }^{+}$in each voxel, instead of the assumed homogeneous transmit field (in principle any kind of $\mathrm{B}_{1}{ }^{+}$measurement can be used). The initial work showed a reduction of the $\mathrm{B}_{1}{ }^{+}$dependence of the $\mathrm{T}_{1}$ maps with increased SNR after the correction. However, in order to increase the image quality and transmit field independence even further in regions that suffer from a low $\mathrm{B}_{1}{ }^{+}$(e.g. the cerebellum and inferior temporal lobes), the standard adiabatic inversion pulse is often replaced by an optimized pulse, such as the time resampled frequency offset corrected inversion (TR-FOCI) pulse (Hurley et al., 2010). Combined with the use of dielectric pads - which are placed strategically between the head and the coil - this significantly improves the inversion efficiency in problematic regions (Teeuwisse et al., 2012; O'Brien et al., 2014). 


\section{CHAPTER 1}

Transversal relaxation $-T_{2}^{(*)}$

The physical source underlying $\mathrm{T}_{2}$ is more complex compared to that of $\mathrm{T}_{1}$. It is defined as the time, for which the transversal magnetization is exponentially decayed to $37 \%$ of the initial value after the excitation and can be assessed from the FID. The high initial FID signal is due to the high phase coherence between the ${ }^{1} \mathrm{H}$ magnetization that was flipped into the $M_{x y}$ plane. This phase coherence decreases quickly because of random fluctuations in the local magnetic field caused by the surrounding tissue environment (i.e. caused by protons and other micro- and mesoscopic field inhomogeneities). In addition, the phase coherence decreases due to susceptibility differences between tissues. In practice, phase decoherence happens faster due to the additional imperfections in the main magnetic field (i.e. macroscopic magnetic field inhomogeneities) inducing differently precessing protons (Yablonskiy and Haacke, 1994; Chavhan et al., 2009). In contrast to $T_{1}$-recovery, $T_{2}$ - or $T_{2}{ }^{*}$-relaxation does not involve energy exchange with the environment.

$\mathrm{T}_{2}{ }^{*}$-decay during an FID experiment consists of an irreversible and reversible component. The reversible component can be refocused by applying an 180 degree pulse at TE/ 2 and the decay constant is termed $T_{2}$. The effective (measured) transversal decay without such a refocusing pulse will therefore always be lower and is annotated differently as $T_{2}{ }^{*}$ (see Table 1.2). The difference between $T_{2}$ and $T_{2}{ }^{*}$ is influenced by the voxel size, as larger voxels suffer more from intravoxel dephasing (Yablonskiy and Haacke, 1994). Because of the mesoscopic field inhomogeneities, both $T_{2}$ and $T_{2}{ }^{*}$ will decrease in voxels with higher iron content, to a lesser extent, myelin and calcium concentrations and deoxygenated blood (Haacke et al., 2005; Stuber et al., 2014). This difference is determined by the superparamagnetic properties of the macromolecules (e.g. high for iron) and blood.

Table 1.2 | Gray (GM), white matter (WM), cerebral spinal fluid (CSF) and blood $T_{2}$ and $T_{2}^{*}$ values (in ms) obtained using 3T and 7TMRI. Data from Uludag, Muller-Bierl et al. (2009) and references therein.

\begin{tabular}{l|l|l|l|l} 
& \multicolumn{4}{c}{$T_{2}$} \\
& 3T & 7T & 3T & 7T \\
\hline GM & $99( \pm 7)$ & $55( \pm 4.1)$ & $66( \pm 1.4)$ & $33.2( \pm 1.3)$ \\
\hline WM & $69( \pm 3)$ & $45.9( \pm 1.9)$ & $53.2( \pm 1.2)$ & $26.8( \pm 1.2)$ \\
\hline CSF & $1429( \pm 475)$ & $946( \pm 206)$ & 333.5 & $168( \pm 12)$ \\
\hline Blood & 32.2 & $55.0( \pm 4.1)$ & 21.2 & $7.4( \pm 1.4)$ \\
\hline
\end{tabular}

Acquisition of whole-brain quantitative $T_{2}$ maps is problematic at high field strengths, as mitigation of macroscopic susceptibility effects is necessary through RF refocusing, similar as it is typically done with spin echo experiments at lower field strengths. $T_{2}$ 
mapping using spin echo at $\mathrm{B}_{0}>3 \mathrm{~T}$ is therefore challenging due to inhomogeneous transmit profiles, power deposition limitations and long acquisition times, potentially posing safety issues related to tissue heating (Duyn, 2012). In contrast, $\mathrm{T}_{2}{ }^{*}$ mapping is straight-forward to implement at high field strengths and requires acquisition of multiple (typically 4-8) $T_{2}{ }^{*} W$ GRE images at different times (TEs in this case) during the transversal decay and typically involves a low flip angle and long TR (Cohen-Adad, 2014). The dephasing effect (and hereby $T_{2}{ }^{*}$ sensitivity) increases with field strength due to increased susceptibility effects, which is directly dependent on $B_{0}$ and the susceptibility of the source producing the field inhomogeneity (Chavhan et al., 2009). After acquiring the data, $\mathrm{T}_{2}{ }^{*}$ can then be quantified by fitting the multi-echo data to a mono-exponential model (see Fig. 1.3):

$$
S(T E)=S_{0} e^{-\frac{T E}{T_{2} *}}
$$

Again, $\mathrm{S}(\mathrm{TE})$ is the signal measured at time $T E$, while $\mathrm{S}_{0}$ is the signal that would be acquired at the magnetic equilibrium. In order to acquire $\mathrm{a}_{2}{ }^{*}$ map of sufficient quality within a reasonable time frame, trade-offs have to be made between sequence parameters, such as the spatial resolution, number of TEs and selected flip angle. While more TEs and a low flip angle will result in a more accurate estimation of $\mathrm{T}_{2}{ }^{*}$, higher spatial resolution requires longer imaging times. Especially in patients, this will increase the occurrence of subject motion, resulting in erroneous estimation of $\mathrm{T}_{2}{ }^{*}$. In addition, an anisotropic voxel size will increase the dependence on the macroscopic field inhomogeneities, as well as partial volume effects in the slice direction (Cohen-Adad, 2014).
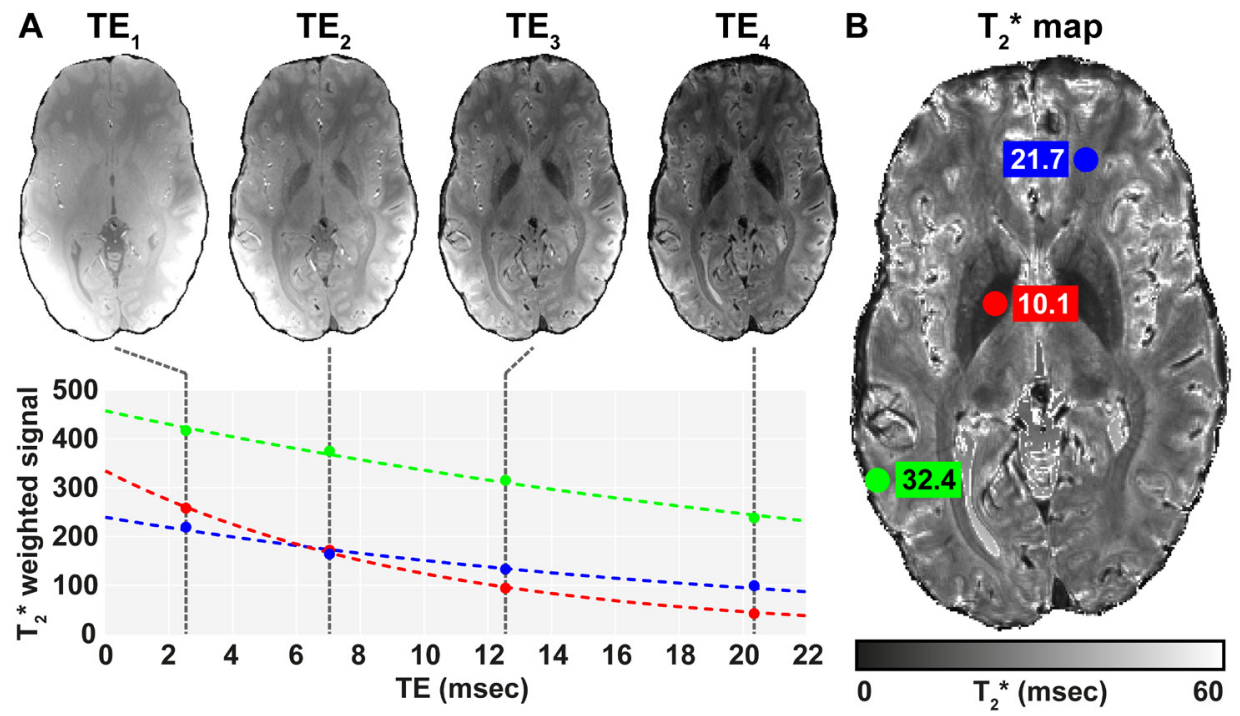

Fig. 1.3. $\mid T_{2}^{*}$ quantification based on ME-GRE data and four TEs (A). Colored dashed lines represent the mono-exponential fit for a single voxel, indicated in B. Values represent the obtained $T_{2}^{*}$. 


\section{CHAPTER 1}

\subsection{2. | Perfusion}

Cerebral perfusion refers to the delivery of oxygen and nutrients (like glucose) to the neurons by means of blood flow and is, therefore, tightly coupled to brain metabolism (Raichle, 1998). In addition to mapping $T_{1}$ and $T_{2}{ }^{*}$, differences in magnetization can be used to quantify cerebral perfusion. Here, the absolute perfusion is proportional to signal changes induced by the change in longitudinal magnetization and/or susceptibility effects of either an exogenous (mostly gadolinium-based) or endogenous (magnetically labeled arterial blood water) tracer, while it passes through the brain's vasculature (Barbier et al., 2001). The first method is invasive and therefore poses a discomfort compared to the relaxometry-related, described above, quantitative methods and will not be discussed further. The latter method, known as arterial spin labeling (ASL), is still under active development in order to be reliably used in the clinic (see the recommendations for standardization in the white paper by Alsop et al. (2015)).

\section{Arterial spin labeling}

ASL data typically includes a time series of images that is characterized by repetitions of control (i.e. non-labeled) and tag (i.e. labeled using an inversion pulse upstream of the imaging slab) image pairs (Williams et al., 1992). Here, the inversion pulse inverts (i.e. 'tags') blood water magnetization flowing through the labeling slab (e.g. carotid arteries in the neck). The inverted magnetization flows into the imaging slab and reduces the overall tissue magnetization due to water exchange with brain tissue water. The signal differences between control and tag images - which are acquired after a specific transit time delay (typically 700 to $2000 \mathrm{~ms}$ ) following the inversion pulse - is therefore proportional to the local tissue perfusion (e.g. cerebral blood flow, CBF, see Fig. 1.4A) and can be quantified using the following equation (Alsop et al., 2015):

$$
C B F=\frac{6000 \cdot \lambda \cdot\left(S_{\text {control }}-S_{\text {tag }}\right) \cdot e^{\frac{T I_{2}}{T_{1, \text { blood }}}}}{2 \cdot \alpha \cdot T I_{1} \cdot M_{0}}
$$

Here, the $T_{1, \text { blood }}$ is field strength specific (see Table 1.), $\lambda$ is the brain/blood partition coefficient (e.g. $0.9 \mathrm{ml} / \mathrm{g}$ ) and a the labeling efficiency (e.g. typically around 0.95). The factor 6000 converts the units from $1 / \mathrm{s}$ to $\mathrm{ml} / 100 \mathrm{~g} / \mathrm{min}$, which is the physiological unit for CBF. Different labeling schemes are available and are either continuous (CASL, using a long, 1-3 s pulse) (Alsop and Detre, 1998), pseudo-continuous (PCASL, train of shorter pulses of $1 \mathrm{~ms}$ each) (Dai et al., 2008) or pulsed (PASL, short pulse or a limited number of pulses, 10-20 ms) (Wong et al., 1998). Main differences across these approaches are the spatial extent and the duration of the labeling (see Fig. 1.4B). 
The choice for labeling scheme depends on field strength, hardware and spatial resolution, as each of the approaches are characterized by their own advantages and disadvantages (Alsop et al., 2015). For example, CASL utilizes a long labeling time, which poses problems related to high power depositions to the tissue and suffers from attenuated free water signal in the imaging slab via magnetization transfer (MT) effects. Since the MT effect is solely present during labeling, this will render the final perfusion map not purely perfusion- but also MT-weighted. Applying an amplitude-modulated RF irradiation during the control image has been proposed to eliminate this (Alsop and Detre, 1998). Alternatively, the CASL-related SAR and MT effect issues can be dealt

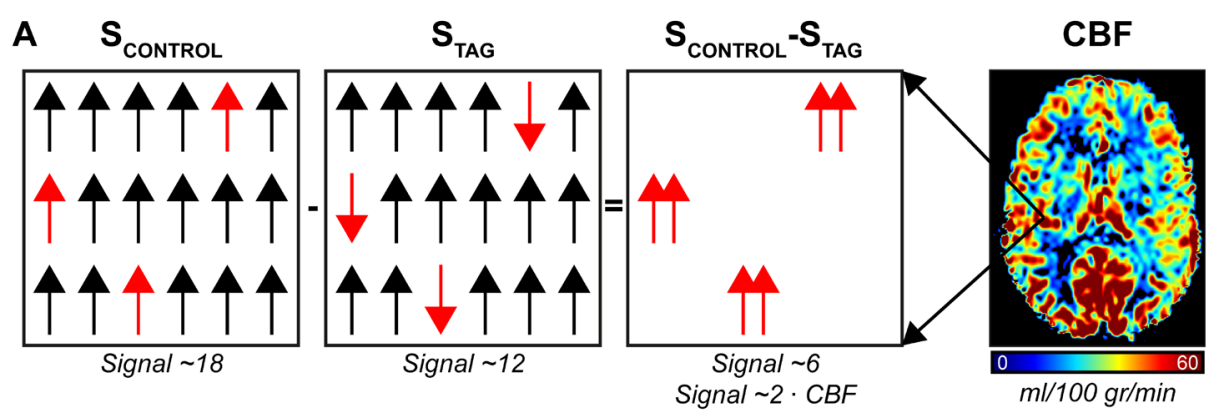

B CONTROL

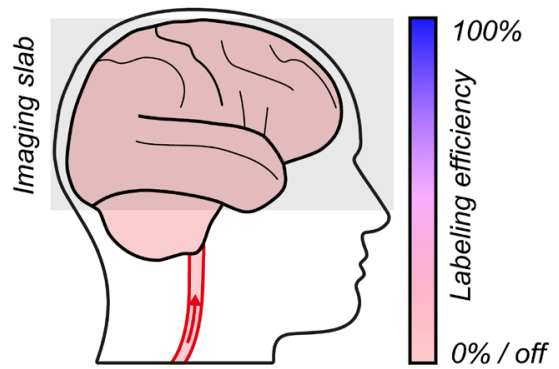

PASL

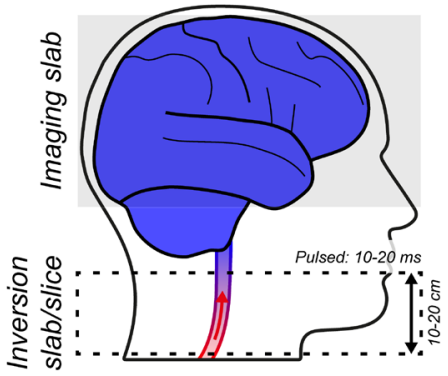

PCASL

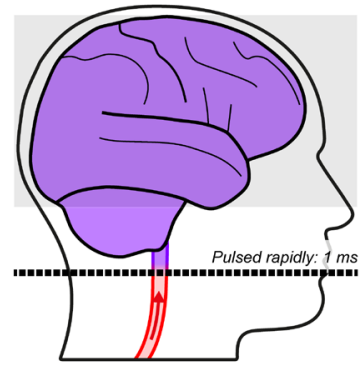

CASL

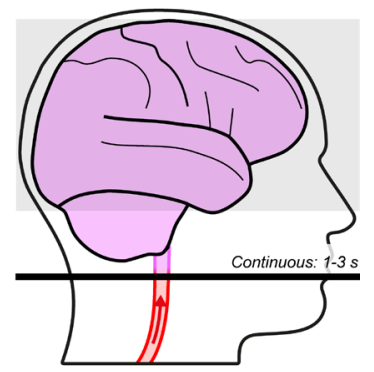

Fig. 1.4. Schematic illustration of the signal obtained from a single voxel with and without magnetically tagging inflowing blood (A). CBF maps are obtained by voxel-wise quantification based on multiple control-tag difference images and the model in Eq. 1.5. Different methods can be used to label the blood. The different labeling schemes and their properties are highlighted in $B$. 


\section{CHAPTER 1}

with by using a PASL scheme, permitting higher inversion efficiency. Flow-sensitive alternating inversion recovery (FAIR) is one the most popular PASL schemes (Kim, 1995). However, PASL is characterized by a lower difference signal between control and tag images and, consequently, lower SNR (compared to CASL) and suffers from arterial transit time delays and inversion slab profile artifacts. The current consensus is that PCASL has the highest SNR and controllable levels of artifacts at 3T, as it combines the best of both CASL (i.e. high SNR) and PASL (i.e. high labeling efficiency) approaches (Alsop et al., 2015). In addition, PCASL was characterized by the highest within-subject reproducibility and comparability with ${ }^{15} \mathrm{O}$-water positron emission tomography measures across all methods (Xu et al., 2010; Chen et al., 2011; Gevers et al., 2011).

Application of ASL at higher field strengths remains technically challenging (Wang et al., 2002; Gardener et al., 2009; Ivanov et al., 2017). Higher field strength improves the temporal SNR (i.e. voxelwise ratio of its mean divided by its standard deviation across time) arising from the increased image SNR (Pohmann et al., 2016) and blood T, (Rooney et al., 2007), allowing acquisition of (relatively) high resolution ( $\leq 2 \mathrm{~mm}^{3}$ ) and whole-brain perfusion-weighted images (Ivanov et al., 2017). As a result and by taking into account that the cortical thickness varies (on average) between 2 and $4 \mathrm{~mm}$, the GM ASL signal is less affected by partial volume effects with CSF and WM. However, challenges lie mainly in maintaining sufficient spatial homogeneity and labeling efficiency, which are hampered by the $\mathrm{B}_{0}$ and $\mathrm{B}_{1}^{+}$inhomogeneities commonly observed at higher field strengths (Teeuwisse et al., 2010). As a result, overestimation of the labeling efficiency and duration, but also the use of suboptimal quantification models at higher field strengths that rely on efficient labeling (i.e. Kety-Schmidt), may result in the lower gray matter perfusion compared to lower field strengths (e.g. 40 vs. 60 $\mathrm{ml} / 100 \mathrm{gr} / \mathrm{min}$ at 7T and 3T, respectively) (Bause et al., 2016; Ivanov et al., 2017). Finally, recent advances showed that UHF ASL can also be used to concurrently measure functional BOLD signal and baseline perfusion, useful for investigating the brain's physiology in health and disease (Ivanov et al., 2017a; 2017b).

\subsection{3. | Other quantitative MRI approaches}

Besides mapping $\mathrm{T}_{1}, \mathrm{~T}_{2}{ }^{(*)}$ and $\mathrm{CBF}$, other quantitative measures can be obtained using MRI. Since these will not be exploited further in this thesis, these are only briefly discussed.

\section{Magnetization transfer (MT)}

MT is a contrast mechanisms induced by the cross-relaxation between macromolecules, predominantly myelin, and their closely associated water molecules (i.e. bound 
water) via dipole-dipole and chemical exchange interactions with the free water protons (i.e. free water pool) (Wolff and Balaban, 1989). This exchange is usually achieved using a specially designed off-resonance MT pulse(s) to apply RF energy exclusively to the bound pool (i.e. bound water and macromolecules). The ratio of two volumes, acquired with or without the MT pulse, provides the MT ratio (MTR) and is mostly used in the clinic. However, the MTR is susceptible to sequence parameters, $T_{1}$ relaxation and flip angle inhomogeneities (Berry et al., 1999). Different models have been proposed to acquire absolute MT images, including for example the two-pool Bloch model (Henkelman et al., 1993). In general, quantitative MT has shown to be very specific and sensitive to myelination changes (Helms et al., 2008).

\section{Diffusion}

Another popular MRI contrast, especially for white matter studies, is based on the displacement of water molecules between the RF excitation and moment of read-out, i.e. diffusion MRI (dMRI) (Le Bihan et al., 1986). Because of the presence of biological barriers, such as membranes and organelles, the water molecules in different tissues can be characterized by their degree of anisotropic movement and main direction. Several parametric maps can be obtained using dMRI, each based on specific diffusion-related tissue characteristics, such as mean diffusivity (MD, average diffusion across all acquired directions) and fractional anisotropy (FA, index for diffusion asymmetry) maps. For example, high FA is observed in white matter because of its coherently structured myelinated axons, while MD is very high in CSF. Most diffusion-weighted scanning approaches utilize the pulsed gradient spin echo sequence (Stejskal and Tanner, 1965) and use several diffusion gradients with varying strengths (i.e. b-value, ranging typically between $700-1000 \mathrm{~s} / \mathrm{mm}^{2}$ ) and directions $( \pm 30-40)$ to acquire diffusion-weighted images. These images are than processed post-hoc to obtain the quantitative parametric maps or track the white matter fiber bundles (i.e. diffusion tensor imaging) (Mori et al., 1999; Minati and Węglarz, 2007).

\section{MR Spectroscopy (MRS)}

While the previously discussed methods provide the magnetic properties (e.g. $T_{1}, T_{2}^{*}$ ) of the tissue within many voxels, ${ }^{1} \mathrm{H}-\mathrm{MRS}$ aims at quantifying the chemical composition of tissue, restricted to a single or very small number of larger voxel(s) (Jansen et al., 2006). Here, Fourier transformation of the voxel's MRS signal, measured using specialized sequences like point-resolved spatially localized spectroscopy (PRESS, Bottomley (1984)) or stimulated-echo acquisition mode (STEAM, Frahm et al. (1987)), reveals the different metabolites present in the volume of interest (VOI). Typical metabolites that are identified using ${ }^{1} \mathrm{H}-\mathrm{MRS}$ include $n$-acetyl-aspartate (NAA), choline (Cho), creatine 


\section{CHAPTER 1}

(Cr), myo-inositol (ml), y-aminobutryric acid (GABA) and glutamate (Glu). NAA, for example, is a marker of neuronal density and viability, and therefore, often studied in neurodegenerative diseases. Each metabolite is positioned differently along the MRS frequency spectrum based on its chemical shift ( $\delta$, in ppm, see Fig. 1.5A), which is determined by the local molecular environment (Proctor and Yu, 1950). The size and shape (i.e. area) of the peak contains information with regards to the metabolite's concentration and is expressed in $\mathrm{mmol} / \mathrm{L}$, after quantification (Minati et al., 2010). However, also other factors affect the geometry of the peaks, including $T_{1}$ (i.e. affecting peak height), and $T_{2}$ and $T_{2}{ }^{*}$ (i.e. line width of the peak) (Jansen et al., 2006) which need to be taken into account. While MRS at higher fields strengths leads to better spectral separation (see Fig. 1.5B), these confounding effects become more prominent and necessitate more specialized sequence designs to overcome these and many other factors (see recent review by Henning (2018)). Although this method is promising for characterization of metabolic changes in disease, we choose not to include it in our final protocol. This was motivated by our focus on multi-parametric mapping (see next section), as well as time constraints.
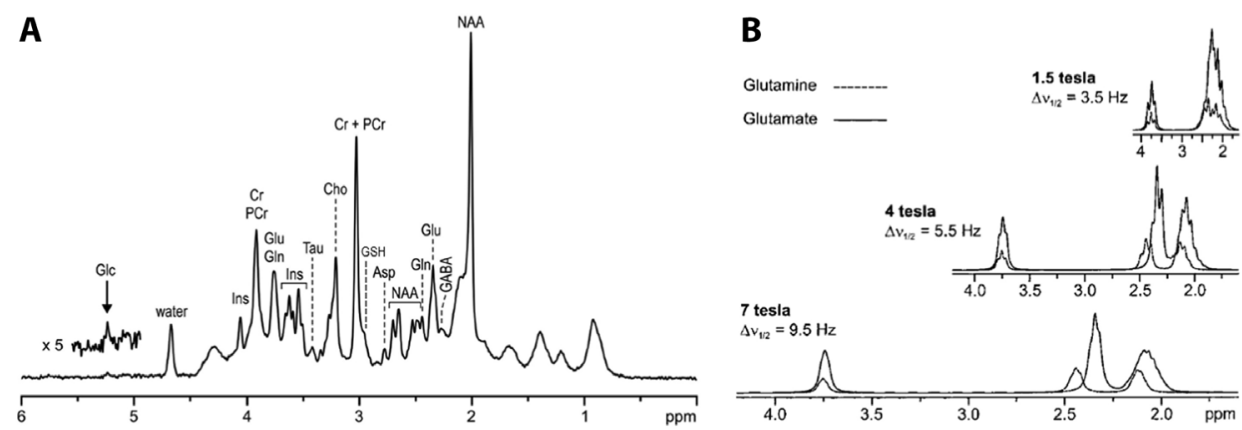

Fig. 1.5. | ${ }^{1} \mathrm{H}-\mathrm{MRS}$ spectrum at $7 T$ acquired using STEAM (A). Glu and Gln separation improves with field strength (B). Figure obtained from Henning (2018).

\section{3. | MULTI-PARAMETRIC QMRI}

As highlighted in the previous sections, MRI can produce different tissue contrasts depending on the magnetization state of the tissue, determined primarily by the sequence setup, such as timing, duration and spatial target of RF pulses. Each contrast is able to capture useful information about specific aspects of the brain's structure or state. That is, when combined in a multi-parametric protocol, more detailed descriptions of the brain's anatomy or inferences about pathological tissue can be achieved (Uludag and Roebroeck, 2014). Here, the choice of qMRI methods and sequences is mostly based on study aims and the available scanning time. Especially for clinical studies, scanning protocols need to be as efficient and short as possible $(<1 \mathrm{hr}$ preferably) to minimize the effects of movement and patient discomfort. To serve this re- 
quirement, multiple parametric maps can be obtained simultaneously using a single scan, instead of serial acquisition of the data (Warntjes et al., 2007; Ma et al., 2013; Weiskopf et al., 2013). However, these approaches have not yet been fully validated at UHF strengths and typically are low spatial resolution. Nevertheless, after acquisition of the data and alignment to a template space, conclusions can be drawn using individual statistical analyses for each parametric map, or after combined statistical testing across all parametric maps (Winkler et al., 2016). As such, multi-parameter qMRI can answer a wide range, from fundamental to purely clinical, research questions. Two

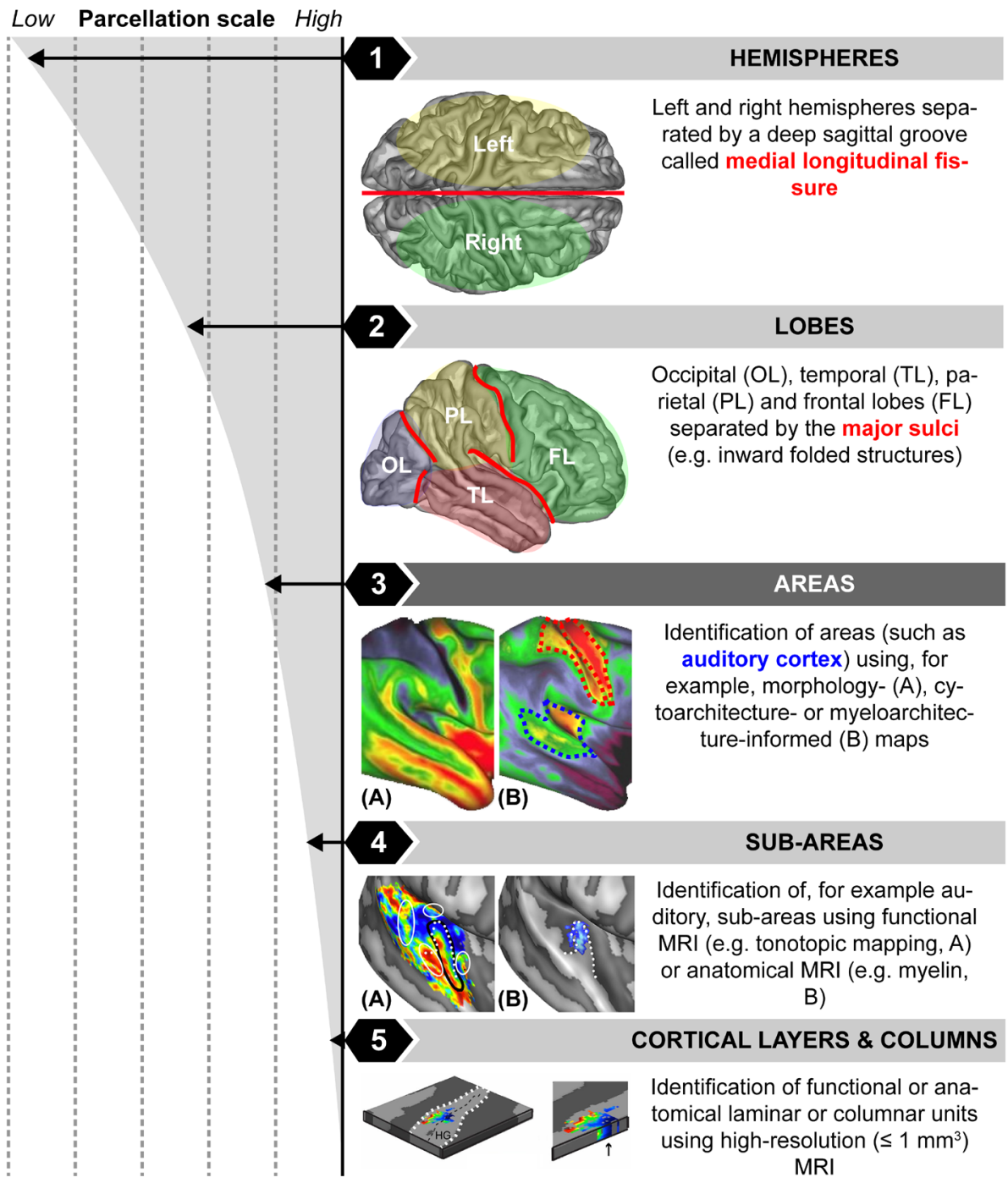

Fig. 1.6. | Brain parcellation scale based on MRI data. The levels of parcellation are sorted from 'low' (top) to 'high' (bottom). The data shown for 3 (i.e. 'areas), 4 (sub-areas) and 5 (cortical layers and columns) are obtained from Glasser and Van Essen (2011), De Martino et al. (2015b) and (2015a), respectively. 
examples, that are relevant for the work in this thesis, are discussed in the following.

\subsection{1. | Cortical parcellation based on myelin and morphology}

The human brain is a complex, but highly structured organ as apparent by its high degree of cortical folding. Whereas the cortical mantle and inner subcortical structures (i.e. GM) contain mainly the neuronal cell bodies, the fiber bundles in the WM form coherent pathways connecting neurons, spatially separated up to many centimeters. It is known that the brain can be subdivided, i.e. parcellated, into distinct meaningful regions based on different functional and anatomical criteria and this is often used as a reference for further analyses. Brain parcellations can be performed at different levels using MRI (see Fig. 1.6), but typically starts from the classification of WM and GM (i.e. macroscopic scale, $\geq 10 \mathrm{~mm}^{3}$ ) towards small spatial processing units (i.e. mesoscopic scale, $<1 \mathrm{~mm}^{3}$ ) (Uludag and Roebroeck, 2014; De Martino et al., 2017).

As such, multi-parametric qMRI can be beneficial to parcellate the different lobes into areas by combining the information from different parametric maps (e.g. level 3 in Fig. 1.6). In this context, in vivo myeloarchitectonics has recently gained a lot of popularity. Recent work, both at lower and UHF strengths, has shown the usefulness of different myelin-weighted MRI contrasts for parcellating cortical GM (Geyer et al., 2011; Glasser and Van Essen, 2011). These contrasts can be used individually (Geyer et al., 2011; Cohen-Adad et al., 2012), by computing a simple ratio image of two contrasting myelin-weighted contrasts, such as $T_{1} w$ and $T_{2}{ }^{(*)} w$ (Glasser and Van Essen, 2011; De Martino et al., 2015) or by more complex combinations using, for example, independent component analysis (Mangeat et al., 2015). Validation studies using surface- and volume-based analyses of the distribution of $M R I T_{1}$ and $T_{2}^{(*)}$-related intensity values across the cortex, including the laminar structure, showed clear correspondence with post-mortem histological myelin (and iron) stainings (see Fig. 1.7) (Bock et al., 2011; Nieuwenhuys, 2013; Stuber et al., 2014). In general, higher myelination is observed in the primary cortical areas, including the sensory regions (i.e. somatosensory, motor, visual and auditory cortices), but also in higher-order regions like the human homologue of the middle temporal gyrus (hMT or V5).

Subsequently, following the myelin-weighted pattern across the cortex, regions can be identified semi-automatically based on intensity thresholds (this thesis) or guided by strong intensity gradients (Glasser and Van Essen, 2011). Please note that the accuracy of a cortical parcellation is partly driven by the correct classification of GM and WM. An incorrect segmentation may mix contributions from multiple tissue classes (i.e. partial volume effects) and may lead to a less accurate estimation of the cortical thickness (i.e. 


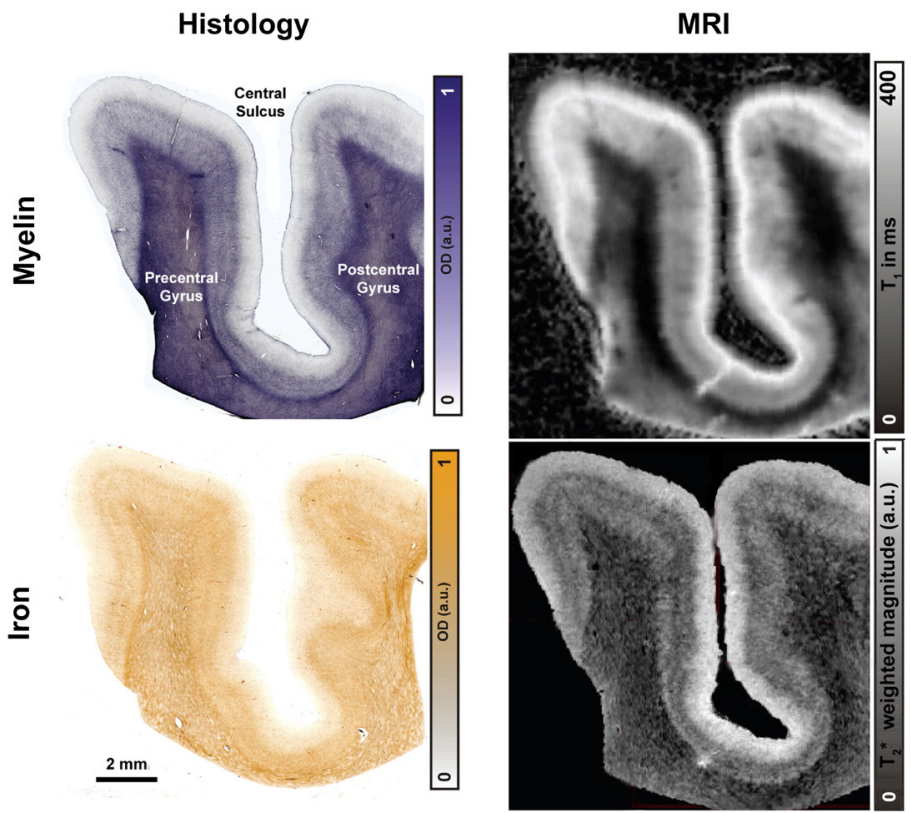

Fig. 1.7. | Myelin- (top) and iron-sensitive (bottom) histological (left) and MRI data (right) of the primary motor- (precentral gyrus)/primary somatosensory (postcentral gyrus) cortices.

distance between WM-GM and GM-CSF borders in $\mathrm{mm}$ ) and subsequent parcellation. This is in particular problematic at UHF strengths with stronger $B_{0}$ and $B_{1}$ inhomogeneities, as discussed above. As such, the effects of $\mathrm{B}_{1}{ }^{+}$correction on $\mathrm{T}_{1}$ and automated estimation of the cortical thickness are evaluated in Chapter 2 of this thesis.

Additional maps, such as cortical thickness or (task or resting-state-based) fMRI maps, can be acquired to incorporate non-myelin-based modalities, as recently introduced by Glasser et al. (2016) using 3T data from the Human Connectome Project (HCP) and a semi-automatic approach based on intensity gradients. Ideally, fully automated surface- or volume-based parcellations or clustering algorithms have to be employed that do not require manual input and accept any type of parametric map, selected based on the type of study and research aims (Thirion et al., 2014). A recent review by Arslan et al. (2017) compared several subject- and group-level-based (single modality) parcellation methods in terms of reproducibility, cluster validity, multi-modal agreement and impact on network analysis. Most of the studies use connectivity information, derived either from fMRI or dMRI data, and varying (e.g. hierarchical, k-means and spectral) clustering algorithms to parcellate the cortex (Eickhoff et al., 2015). These algorithms separate clusters of vertices or voxels that are characterized by different connectivity patterns by applying specific criteria, such as through minimization of the average distance between the cluster's data points and centroid in 


\section{CHAPTER 1}

k-means clustering. Also here, the specific research question determines, which clustering algorithm is the most appropriate. For example, the hierarchical Ward clustering method requires that two clusters are spatially connected in order to be merged (Ward Jr, 1963). This method seems more applicable for microstructure (e.g. myelin)-based parcellations compared to that based on functional connectivity, as larger inter-cluster distance does not directly implicate lower inter-cluster linkage (e.g. in case of stronger connectivity to more distant instead of neighboring regions).

\subsection{2. | Moving towards clinical ultra-high field MRI}

UHF MRI has shown its potential for neuroimaging research and therefore led to the recent approval of 7T MRI for clinical diagnosis world-wide ${ }^{1}$. Several neuroimaging studies highlighted the range of spatial and temporal resolutions that can be achieved with in vivo 7T imaging (Pfeuffer et al., 2002; Yacoub et al., 2008; Gardener et al., 2009; Stuber et al., 2014; Ivanov et al., 2017; Keuken et al., 2017; Uludag and Blinder, 2017). As indicated above, 7T data is characterized by improved SNR and CNR. This provides opportunities to increase spatial resolution, which is crucial for earlier detection of fine pathological lesions or to reduce scanning time to minimize motion artifacts. Although the majority of studies are done in healthy subjects, more and more studies focus on specific clinical populations, such as multiple sclerosis (MS), Alzheimer's disease (AD), but also cerebrovascular disease and brain tumor patients. Early studies already provided clear evidence of the enhanced detection of disease-specific morphological changes in, for example, MS and AD using 7T compared to 3T (Nakada et al., 2008; Kollia et al., 2009). Studies, comparing lower to UHF strength MRI for a wide range of clinical applications, have been discussed recently by Trattnig et al. (2016). In this context, quantitative MRI may be especially beneficial, since $T_{1}, T_{2}{ }^{*}, C B F$ or other tissue parameters can be directly compared with those observed in healthy controls, across patients but also across multiple imaging sites and scanner vendors without the need for any normalization. For example, GM lesions in MS patients are characterized by an increased $\mathrm{T}_{2}{ }^{*}$ compared to normal-appearing $\mathrm{GM}$, consistent with cortical myelin and iron loss (Mainero et al., 2015; Jonkman et al., 2016). In addition, multi-parametric qMRI using anatomical contrasts, such as $\mathrm{T}_{1}$ and $\mathrm{T}_{2}{ }^{*}$, allows detailed delineation of subcortical structures, as outlined in Fig. 1.8, necessary for correct and selective targeting of deep-brain stimulation surgery in Parkinson's patients (Plantinga et al., 2014). scanner. It comes with Dual Mode functionality users: research vs. clinical use. FDA and CE approval has been granted for clinical use on October $12^{\text {th }} 2018$ for North-America and August $22^{\text {nd }} 2017$ for Europa, respectively. 


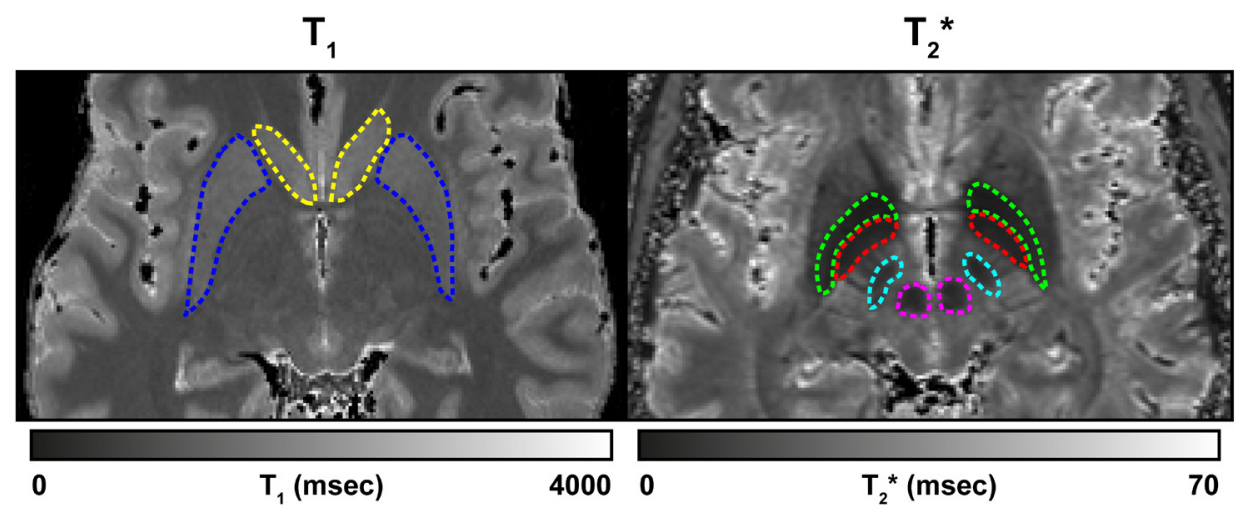

Fig. 1.8. | Examples of subcortical structures identified using quantitative $T_{1}$ and $T_{2}^{*}$ maps. Yellow, blue, green, red, cyan and magenta dashed outlines indicate the caudate nucleus, putamen, globus pallidus externa, globus pallidus interna, substantia nigra and red nucleus, respectively.

Fundamentally, UHF imaging must be robust in order to accommodate its widespread use. Here, an important aspect is the within- and between-subject reproducibility of the data (such as in this thesis, Jansen et al. (2007), Marques et al. (2010), Chen et al. (2011) and Govindarajan et al. (2015)). In that sense, qMRI is expected to be fairly robust, as quantitative values better represent the ground truth (i.e. properties or state of the tissue), compared to the conventional clinical sequences. However, as mentioned earlier, especially at higher field strengths qMRI is not necessarily free from effects, which are unrelated to the underlying microstructural properties or perfusion of the brain. Variations in qMRI volume or surface maps can be of hardware- (e.g. dayto-day variability, coil loading, shimming, slice positioning and patient motion) or software-related (scanning software release patches, as well as post-hoc processing methods, e.g segmentation, fitting, registration and surface mapping procedures) origin. In summary, UHF imaging provides new windows for the detection of neuropathological changes in disease, due to the increased sensitivity mediated by the higher spatial resolution and contrast.

\section{4. | AIMS AND OUTLINE OF THIS THESIS}

The aim of the work presented in the current thesis is two-fold:

\section{- Evaluation and application of a quantitative brain 7T protocol suitable both for healthy subjects and for patients \\ - Identification of MRI (and genetic) biomarkers of the diseased brain using this protocol.}

The specific aspects and details of the protocol are highlighted throughout the different chapters. 


\section{CHAPTER 1}

Surface-based analyses of cortical properties, including morphology, microstructure and perfusion, depend on the quality of the anatomical data. Therefore, in Chapter $\mathbf{2}$, we characterize the performance of automatic segmentation software (i.e. FreeSurfer) based on original and $\mathrm{B}_{1}^{+}$-corrected MP2RAGE data by quantifying the differences in measured cortical thickness. In addition, we evaluate the change in apparent $\mathrm{T}_{1}$ before and after this correction. In Chapter 3, we perform a systematic comparison between several MRI-based approaches to map cortical myelin content in vivo. Quantitative and weighted $\mathrm{T}_{1}, \mathrm{~T}_{2}{ }^{*}$ and their ratio surface maps are compared in terms of 'parcellability', CNR and inter- and intra-subject variability and reproducibility. In Chapter 4, we present quantitative data, including $T_{1}, T_{2}{ }^{*}$, as well as CBF maps, in m.3243A>G patients. These patients are characterized by a specific mitochondrial mutation, which causes a widely varying phenotype. Volume- and surface-based analyses are used to compare their brain's state across patients as a function of genetic load, as well as group comparison with healthy controls. Chapter $\mathbf{5}$ extents the methods used in Chapter 4 towards a larger-scale imaging genetics study, focusing on Type 2 Diabetes Mellitus. We propose analysis strategies that combine information from multiple data modalities, including MRI, whole-exome sequencing and metabolomics, and identify biomarkers that enable characterization of subgroups within the study population. Finally, Chapter 6 includes a summary of the empirical chapters and a discussion centered on future implications and developments related to the presented work. 


\section{5. | REFERENCES}

Alsop, D. C. and J. A. Detre (1998). “Multisection cerebral blood flow MR imaging with continuous arterial spin labeling." Radiology 208(2): 410-416.

Alsop, D. C., J. A. Detre, X. Golay, M. Gunther, J. Hendrikse, L. Hernandez-Garcia, .. . G. Zaharchuk (2015). "Recommended implementation of arterial spin-labeled perfusion MRI for clinical applications: A consensus of the ISMRM perfusion study group and the European consortium for ASL in dementia." Magn Reson Med 73(1): 102-116.

Arslan, S., S. I. Ktena, A. Makropoulos, E. C. Robinson, D. Rueckert and S. Parisot (2017). “Human brain mapping: A systematic comparison of parcellation methods for the human cerebral cortex." Neuroimage.

Barbier, E. L., L. Lamalle and M. Decorps (2001). "Methodology of brain perfusion imaging." J Magn Reson Imaging 13(4): 496-520.

Bause, J., P. Ehses, C. Mirkes, G. Shajan, K. Scheffler and R. Pohmann (2016). "Quantitative and functional pulsed arterial spin labeling in the human brain at 9.4 T." Magn Reson Med 75(3): 1054-1063.

Berry, I., G. J. Barker, F. Barkhof, A. Campi, V. Dousset, J. M. Franconi, ... P. S. Tofts (1999). "A multicenter measurement of magnetization transfer ratio in normal white matter." J Magn Reson Imaging 9(3): 441-446.

Bloch, F. (1946). “Nuclear induction.” Physical review 70(7-8): 460.

Bock, N. A., E. Hashim, A. Kocharyan and A. C. Silva (2011). "Visualizing myeloarchitecture with magnetic resonance imaging in primates." Ann N Y Acad Sci 1225 Suppl 1: E171-181.

Bottomley, P. A. (1984). Selective volume method for performing localized NMR spectroscopy, Google Patents.

Bydder, G. M. and I. R. Young (1985). "MR imaging: clinical use of the inversion recovery sequence." J Comput Assist Tomogr 9(4): 659-675.

Chavhan, G. B., P. S. Babyn, B. Thomas, M. M. Shroff and E. M. Haacke (2009). "Principles, techniques, and applications of T2*-based MR imaging and its special applications." Radiographics 29(5): 1433-1449.

Chen, Y., D. J. Wang and J. A. Detre (2011). "Test-retest reliability of arterial spin labeling with common labeling strategies." J Magn Reson Imaging 33(4): 940-949.

Cohen-Adad, J. (2014). "What can we learn from T2* maps of the cortex?" Neuroimage 93 Pt 2: 189-200.

Cohen-Adad, J., J. R. Polimeni, K. G. Helmer, T. Benner, J. A. McNab, L. L. Wald, .. . C. Mainero (2012). " $\mathrm{T}(2)^{*}$ mapping and $\mathrm{B}(0)$ orientation-dependence at $7 \mathrm{~T}$ reveal cyto- and myeloarchitecture organization of the human cortex." Neuroimage 60(2): 1006-1014.

Dai, W., D. Garcia, C. de Bazelaire and D. C. Alsop (2008). "Continuous flow-driven inversion for arterial spin labeling using pulsed radio frequency and gradient fields." Magn Reson Med 60(6): 1488-1497.

Damadian, R. (1974). Apparatus and method for detecting cancer in tissue, Google Patents.

De Martino, F., M. Moerel, K. Ugurbil, R. Goebel, E. Yacoub and E. Formisano (2015). “Frequency preference and attention effects across cortical depths in the human primary auditory cortex." Proc Natl Acad Sci U S A 112(52): 16036-16041.

De Martino, F., M. Moerel, J. Xu, P. F. van de Moortele, K. Ugurbil, R. Goebel, ... E. Formisano (2015). "High-Resolution Mapping of Myeloarchitecture In Vivo: Localization of Auditory Areas in the Human Brain." Cereb Cortex 25(10): 3394-3405.

De Martino, F., E. Yacoub, V. Kemper, M. Moerel, K. Uludag, P. De Weerd, ... E. Formisano (2017). "The impact of ultra-high field MRI on cognitive and computational neuroimaging." Neuroim- 


\section{CHAPTER 1}

age.

Duyn, J. H. (2012). "The future of ultra-high field MRI and fMRI for study of the human brain." Neuroimage 62(2): 1241-1248.

Duyn, J. H., P. van Gelderen, T. Q. Li, J. A. de Zwart, A. P. Koretsky and M. Fukunaga (2007). “Highfield MRI of brain cortical substructure based on signal phase." Proc Natl Acad Sci U S A 104(28): 11796-11801.

Edzes, H. T. and E. T. Samulski (1977). "Cross relaxation and spin diffusion in the proton NMR or hydrated collagen." Nature 265(5594): 521-523.

Eggenschwiler, F., T. Kober, A. W. Magill, R. Gruetter and J. P. Marques (2012). "SA2RAGE: a new sequence for fast B1+ -mapping." Magn Reson Med 67(6): 1609-1619.

Eickhoff, S. B., B. Thirion, G. Varoquaux and D. Bzdok (2015). “Connectivity-based parcellation: Critique and implications." Hum Brain Mapp 36(12): 4771-4792.

Ernst, R. R. and W. A. Anderson (1966)."Application of Fourier transform spectroscopy to magnetic resonance." Review of Scientific Instruments 37(1): 93-102.

Frahm, J., K.-D. Merboldt and W. Hänicke (1987). "Localized proton spectroscopy using stimulated echoes." Journal of Magnetic Resonance (1969) 72(3): 502-508.

Gardener, A. G., P. A. Gowland and S. T. Francis (2009). "Implementation of quantitative perfusion imaging using pulsed arterial spin labeling at ultra-high field." Magn Reson Med 61(4): 874-882.

Gevers, S., M. J. van Osch, R. P. Bokkers, D. A. Kies, W. M. Teeuwisse, C. B. Majoie, ... A. J. Nederveen (2011). "Intra- and multicenter reproducibility of pulsed, continuous and pseudo-continuous arterial spin labeling methods for measuring cerebral perfusion." J Cereb Blood Flow Metab 31(8): 1706-1715.

Geyer, S., M. Weiss, K. Reimann, G. Lohmann and R. Turner (2011). "Microstructural Parcellation of the Human Cerebral Cortex - From Brodmann's Post-Mortem Map to in vivo Mapping with HighField Magnetic Resonance Imaging." Front Hum Neurosci 5: 19.

Glasser, M. F., T. S. Coalson, E. C. Robinson, C. D. Hacker, J. Harwell, E. Yacoub, . . D. C. Van Essen (2016). "A multi-modal parcellation of human cerebral cortex." Nature 536(7615): 171-178.

Glasser, M. F. and D. C. Van Essen (2011). “Mapping human cortical areas in vivo based on myelin content as revealed by T1- and T2-weighted MRI." J Neurosci 31(32): 11597-11616.

Govindarajan, S. T., J. Cohen-Adad, M. P. Sormani, A. P. Fan, C. Louapre and C. Mainero (2015). "Reproducibility of T2* mapping in the human cerebral cortex in vivo at 7 tesla MRI." J Magn Reson Imaging 42(2): 290-296.

Gowland, P. and P. Mansfield (1993). "Accurate measurement of T1 in vivo in less than 3 seconds using echo-planar imaging." Magn Reson Med 30(3): 351-354.

Haacke, E. M., N. Y. Cheng, M. J. House, Q. Liu, J. Neelavalli, R. J. Ogg, . . . A. Obenaus (2005). "Imaging iron stores in the brain using magnetic resonance imaging." Magn Reson Imaging 23(1): 1-25.

Helms, G., H. Dathe, K. Kallenberg and P. Dechent (2008). “High-resolution maps of magnetization transfer with inherent correction for RF inhomogeneity and T1 relaxation obtained from 3D FLASH MRI." Magn Reson Med 60(6): 1396-1407.

Henkelman, R. M., X. Huang, Q. S. Xiang, G. J. Stanisz, S. D. Swanson and M. J. Bronskill (1993). "Quantitative interpretation of magnetization transfer." Magn Reson Med 29(6): 759-766.

Henning, A. (2018). "Proton and multinuclear magnetic resonance spectroscopy in the human brain at ultra-high field strength: A review." Neuroimage 168: 181-198.

Hurley, A. C., A. Al-Radaideh, L. Bai, U. Aickelin, R. Coxon, P. Glover and P. A. Gowland (2010). "Tailored RF pulse for magnetization inversion at ultrahigh field." Magn Reson Med 63(1): 51-58.

Ivanov, D., A. Gardumi, R. A. M. Haast, J. Pfeuffer, B. A. Poser and K. Uludag (2017a). “Comparison of 3T and 7T ASL techniques for concurrent functional perfusion and BOLD studies." Neuroimage 156: 363-376. 
Ivanov, D., B. A. Poser, L. Huber, J. Pfeuffer and K. Uludag (2017b). “Optimization of simultaneous multislice EPI for concurrent functional perfusion and BOLD signal measurements at 7T." Magn Reson Med 78(1): 121-129.

Jansen, J. F., W. H. Backes, K. Nicolay and M. E. Kooi (2006). "1H MR spectroscopy of the brain: absolute quantification of metabolites." Radiology 240(2): 318-332.

Jansen, J. F., M. E. Kooi, A. G. Kessels, K. Nicolay and W. H. Backes (2007). "Reproducibility of quantitative cerebral T2 relaxometry, diffusion tensor imaging, and $1 \mathrm{H}$ magnetic resonance spectroscopy at 3.0 Tesla." Invest Radiol 42(6): 327-337.

Jonkman, L. E., L. Fleysher, M. D. Steenwijk, J. A. Koeleman, T. P. de Snoo, F. Barkhof, ... J. J. Geurts (2016). "Ultra-high field MTR and qR2* differentiates subpial cortical lesions from normal-appearing gray matter in multiple sclerosis." Mult Scler 22(10): 1306-1314.

Kashyap, S., D. Ivanov, M. Havlicek, B. A. Poser and K. Uludag (2017). "Impact of acquisition and analysis strategies on cortical depth-dependent fMRI." Neuroimage.

Keuken, M. C., P. L. Bazin, K. Backhouse, S. Beekhuizen, L. Himmer, A. Kandola, .. . B. U. Forstmann (2017). "Effects of aging on $\mathrm{T}_{1}, \mathrm{~T}_{2}{ }^{*}$, and QSM MRI values in the subcortex." Brain Struct Funct.

Kim, S. G. (1995). "Quantification of relative cerebral blood flow change by flow-sensitive alternating inversion recovery (FAIR) technique: application to functional mapping." Magn Reson Med 34(3): 293-301.

Koenig, S. H. and R. D. Brown (1987). "Relaxometry of tissue." eMagRes.

Koenig, S. H., R. D. Brown, 3rd, M. Spiller and N. Lundbom (1990). "Relaxometry of brain: why white matter appears bright in MRI." Magn Reson Med 14(3): 482-495.

Kollia, K., S. Maderwald, N. Putzki, M. Schlamann, J. M. Theysohn, O. Kraff, ... I. Wanke (2009). “First clinical study on ultra-high-field MR imaging in patients with multiple sclerosis: comparison of 1.5T and 7T." AJNR Am J Neuroradiol 30(4): 699-702.

Larmor, J. (1897). "LXIII. On the theory of the magnetic influence on spectra; and on the radiation from moving ions." The London, Edinburgh, and Dublin Philosophical Magazine and Journal of Science 44(271): 503-512.

Lauterbur, P. (1973). "Image formation by induced local interactions: examples employing nuclear magnetic resonance."

Lauterbur, P. C. (1989). "Image formation by induced local interactions. Examples employing nuclear magnetic resonance. 1973." Clin Orthop Relat Res(244): 3-6.

Le Bihan, D., E. Breton, D. Lallemand, P. Grenier, E. Cabanis and M. Laval-Jeantet (1986). "MR imaging of intravoxel incoherent motions: application to diffusion and perfusion in neurologic disorders." Radiology 161(2): 401-407.

Look, D. C. and D. R. Locker (1970). "Time saving in measurement of NMR and EPR relaxation times." Review of Scientific Instruments 41(2): 250-251.

Ma, D., V. Gulani, N. Seiberlich, K. Liu, J. L. Sunshine, J. L. Duerk and M. A. Griswold (2013). "Magnetic resonance fingerprinting." Nature 495(7440): 187-192.

Mainero, C., C. Louapre, S. T. Govindarajan, C. Gianni, A. S. Nielsen, J. Cohen-Adad, . . R. P. Kinkel (2015). "A gradient in cortical pathology in multiple sclerosis by in vivo quantitative 7 T imaging." Brain 138(Pt 4): 932-945.

Mangeat, G., S. T. Govindarajan, C. Mainero and J. Cohen-Adad (2015)."Multivariate combination of magnetization transfer, $\mathrm{T}^{*}$ and $\mathrm{B} 0$ orientation to study the myelo-architecture of the in vivo human cortex." Neuroimage 119: 89-102.

Mansfield, P. and P. K. Grannell (1973). “NMR'diffraction'in solids?" Journal of Physics C: solid state physics 6(22): L422.

Marques, J. P. and R. Gruetter (2013). "New developments and applications of the MP2RAGE sequence--focusing the contrast and high spatial resolution R1 mapping." PLoS One 8(7): e69294.

Marques, J. P., T. Kober, G. Krueger, W. van der Zwaag, P. F. Van de Moortele and R. Gruetter (2010). 


\section{CHAPTER 1}

"MP2RAGE, a self bias-field corrected sequence for improved segmentation and T1-mapping at high field." Neuroimage 49(2): 1271-1281.

Minati, L., D. Aquino, M. G. Bruzzone and A. Erbetta (2010). "Quantitation of normal metabolite concentrations in six brain regions by in-vivo 1H-MR spectroscopy." Journal of medical physics/ Association of Medical Physicists of India 35(3): 154.

Minati, L. and W. P. Węglarz (2007). "Physical foundations, models, and methods of diffusion magnetic resonance imaging of the brain: A review." Concepts in Magnetic Resonance Part A 30(5): 278-307.

Mori, S., B. J. Crain, V. P. Chacko and P. C. van Zijl (1999). "Three-dimensional tracking of axonal projections in the brain by magnetic resonance imaging." Ann Neurol 45(2): 265-269.

Moser, E., F. Stahlberg, M. E. Ladd and S. Trattnig (2012). "7-T MR--from research to clinical applications?" NMR Biomed 25(5): 695-716.

Mugler, J. P., 3rd and J. R. Brookeman (1990). “Three-dimensional magnetization-prepared rapid gradient-echo imaging (3D MP RAGE)." Magn Reson Med 15(1): 152-157.

Nakada, T., H. Matsuzawa, H. Igarashi, Y. Fujii and I. L. Kwee (2008). "In vivo visualization of senile-plaque-like pathology in Alzheimer's disease patients by MR microscopy on a 7T system." J Neuroimaging 18(2): 125-129.

Nieuwenhuys, R. (2013). "The myeloarchitectonic studies on the human cerebral cortex of the Vogt-Vogt school, and their significance for the interpretation of functional neuroimaging data." Brain Struct Funct 218(2): 303-352.

Norris, D. G. (2003). “High field human imaging.” J Magn Reson Imaging 18(5): 519-529.

O'Brien, K. R., A. W. Magill, J. Delacoste, J. P. Marques, T. Kober, H. P. Fautz, ... G. Krueger (2014). “Dielectric pads and low- B1+ adiabatic pulses: complementary techniques to optimize structural T1 w whole-brain MP2RAGE scans at 7 tesla." J Magn Reson Imaging 40(4): 804-812.

Ogawa, S., T. M. Lee, A. R. Kay and D. W. Tank (1990). "Brain magnetic resonance imaging with contrast dependent on blood oxygenation." Proc Natl Acad Sci U S A 87(24): 9868-9872.

Pfeuffer, J., P. F. van de Moortele, E. Yacoub, A. Shmuel, G. Adriany, P. Andersen, ... X. Hu (2002). "Zoomed functional imaging in the human brain at 7 Tesla with simultaneous high spatial and high temporal resolution." Neuroimage 17(1): 272-286.

Plantinga, B. R., Y. Temel, A. Roebroeck, K. Uludag, D. Ivanov, M. L. Kuijf and B. M. Ter Haar Romenij (2014). "Ultra-high field magnetic resonance imaging of the basal ganglia and related structures." Front Hum Neurosci 8: 876.

Pohmann, R., O. Speck and K. Scheffler (2016). "Signal-to-noise ratio and MR tissue parameters in human brain imaging at 3, 7, and 9.4 tesla using current receive coil arrays." Magn Reson Med 75(2): 801-809.

Proctor, W. and F. Yu (1950). "The dependence of a nuclear magnetic resonance frequency upon chemical compound." Physical Review 77(5): 717.

Purcell, E. M., H. C. Torrey and R. V. Pound (1946). “Resonance absorption by nuclear magnetic moments in a solid." Physical review 69(1-2): 37.

Raichle, M. E. (1998). "Behind the scenes of functional brain imaging: a historical and physiological perspective." Proc Natl Acad Sci U S A 95(3): 765-772.

Rooney, W. D., G. Johnson, X. Li, E. R. Cohen, S. G. Kim, K. Ugurbil and C. S. Springer, Jr. (2007). "Magnetic field and tissue dependencies of human brain longitudinal $1 \mathrm{H} 2 \mathrm{O}$ relaxation in vivo." Magn Reson Med 57(2): 308-318.

Stejskal, E. O. and J. E. Tanner (1965). “Spin diffusion measurements: spin echoes in the presence of a time-dependent field gradient." The journal of chemical physics 42(1): 288-292.

Stuber, C., M. Morawski, A. Schafer, C. Labadie, M. Wahnert, C. Leuze, ... R. Turner (2014). “Myelin and iron concentration in the human brain: a quantitative study of MRI contrast." Neuroimage 93 Pt 1: 95-106. 
Teeuwisse, W. M., W. M. Brink, K. N. Haines and A. G. Webb (2012). "Simulations of high permittivity materials for $7 \mathrm{~T}$ neuroimaging and evaluation of a new barium titanate-based dielectric." Magn Reson Med 67(4): 912-918.

Teeuwisse, W. M., A. G. Webb and M. J. van Osch (2010). "Arterial spin labeling at ultra-high field: All that glitters is not gold." International Journal of Imaging Systems and Technology 20(1): 62-70.

Thirion, B., G. Varoquaux, E. Dohmatob and J. B. Poline (2014). “Which fMRI clustering gives good brain parcellations?" Front Neurosci 8: 167.

Tofts, P. (2005). Quantitative MRI of the brain: measuring changes caused by disease, John Wiley \& Sons.

Trattnig, S., E. Springer, W. Bogner, G. Hangel, B. Strasser, B. Dymerska, .. S. D. Robinson (2016). "Key clinical benefits of neuroimaging at 7T." Neuroimage.

Twieg, D. B. (1983). "The k-trajectory formulation of the NMR imaging process with applications in analysis and synthesis of imaging methods." Med Phys 10(5): 610-621.

Ugurbil, K. (2017). “Imaging at ultrahigh magnetic fields: History, challenges, and solutions.” $\underline{\text { Neu- }}$ roimage.

Uludag, K. and P. Blinder (2017). "Linking brain vascular physiology to hemodynamic response in ultra-high field MRI." Neuroimage.

Uludag, K., B. Muller-Bierl and K. Ugurbil (2009). "An integrative model for neuronal activity-induced signal changes for gradient and spin echo functional imaging." Neuroimage 48(1): 150-165.

Uludag, K. and A. Roebroeck (2014). "General overview on the merits of multimodal neuroimaging data fusion." Neuroimage 102 Pt 1: 3-10.

Van de Moortele, P. F., E. J. Auerbach, C. Olman, E. Yacoub, K. Ugurbil and S. Moeller (2009). "T1 weighted brain images at 7 Tesla unbiased for Proton Density, T2* contrast and RF coil receive B1 sensitivity with simultaneous vessel visualization." Neuroimage 46(2): 432-446.

Vaughan, J. T., M. Garwood, C. M. Collins, W. Liu, L. DelaBarre, G. Adriany, ... K. Ugurbil (2001). "7T vs. 4T: RF power, homogeneity, and signal-to-noise comparison in head images." Magn Reson Med 46(1): 24-30.

Vaughan, T., L. DelaBarre, C. Snyder, J. Tian, C. Akgun, D. Shrivastava, ... K. Ugurbil (2006). "9.4T human MRI: preliminary results." Magn Reson Med 56(6): 1274-1282.

Wang, J., D. C. Alsop, L. Li, J. Listerud, J. B. Gonzalez-At, M. D. Schnall and J. A. Detre (2002). “Comparison of quantitative perfusion imaging using arterial spin labeling at 1.5 and 4.0 Tesla." Magn Reson Med 48(2): 242-254.

Ward Jr, J. H. (1963). "Hierarchical grouping to optimize an objective function." Journal of the American statistical association 58(301): 236-244.

Warntjes, J. B., O. Dahlqvist and P. Lundberg (2007). "Novel method for rapid, simultaneous T1, T2*, and proton density quantification." Magn Reson Med 57(3): 528-537.

Weiskopf, N., J. Suckling, G. Williams, M. M. Correia, B. Inkster, R. Tait, .. A. Lutti (2013). “Quantitative multi-parameter mapping of $\mathrm{R} 1, \mathrm{PD}\left({ }^{*}\right), \mathrm{MT}$, and $\mathrm{R} 2\left(^{*}\right)$ at $3 \mathrm{~T}$ : a multi-center validation." Front Neurosci 7: 95.

Williams, D. S., J. A. Detre, J. S. Leigh and A. P. Koretsky (1992). "Magnetic resonance imaging of perfusion using spin inversion of arterial water." Proc Natl Acad Sci U S A 89(1): 212-216.

Winkler, A. M., M. A. Webster, J. C. Brooks, I. Tracey, S. M. Smith and T. E. Nichols (2016). “Non-parametric combination and related permutation tests for neuroimaging." Hum Brain Mapp 37(4): 1486-1511.

Wolff, S. D. and R. S. Balaban (1989). "Magnetization transfer contrast (MTC) and tissue water proton relaxation in vivo." Magn Reson Med 10(1): 135-144.

Wong, E. C., R. B. Buxton and L. R. Frank (1998). “Quantitative imaging of perfusion using a single 


\section{CHAPTER 1}

subtraction (QUIPSS and QUIPSS II)." Magn Reson Med 39(5): 702-708.

Xu, G., H. A. Rowley, G. Wu, D. C. Alsop, A. Shankaranarayanan, M. Dowling, . . S. C. Johnson (2010). "Reliability and precision of pseudo-continuous arterial spin labeling perfusion MRI on 3.0T and comparison with 150-water PET in elderly subjects at risk for Alzheimer's disease." NMR Biomed 23(3): 286-293.

Yablonskiy, D. A. and E. M. Haacke (1994). "Theory of NMR signal behavior in magnetically inhomogeneous tissues: the static dephasing regime." Magn Reson Med 32(6): 749-763.

Yacoub, E., N. Harel and K. Ugurbil (2008). "High-field fMRI unveils orientation columns in humans." Proc Natl Acad Sci U SA 105(30): 10607-10612.

Zhang, Y., H. N. Yeung, M. O'Donnell and P. L. Carson (1998). “Determination of sample time for T1 measurement." J Magn Reson Imaging 8(3): 675-681. 


\section{THE EFFECT OF $\mathrm{B}_{1}^{+}$CORRECTION ON MP2RAGE T, AND APPARENT CORTICAL THICKNESS AT 7 TESLA}

ROY A.M. HAAST

DIMO IVANOV

\& KÂMIL ULUDAĞ

Corresponding publication:

"The Effect of $\mathrm{B}_{1}{ }^{+}$Correction on MP2RAGE $\mathrm{T}_{1}$ and Apparent Cortical Thickness at 7 Tesla". Hum. Brain Mapp. 2018 Jun;39(6). 


\section{CHAPTER 2}

\section{ABSTRACT}

Determination of cortical thickness using MRI has often been criticized due to the presence of various error sources. Specifically, anatomical MRI relying on $T_{1}$ contrast may be unreliable due to spatially variable image contrast between gray matter (GM), white matter (WM) and cerebrospinal fluid (CSF). Especially at ultra-high field ( $\geq 7 \mathrm{~T}$ ) $\mathrm{MRI}$, transmit and receive $\mathrm{B}_{1}$-related image inhomogeneities can hamper correct classification of tissue types. In the current paper, we demonstrate that residual $\mathrm{B}_{1}^{+}$ (transmit) inhomogeneities in the $T_{1}$-weighted and quantitative $T_{1}$ images using the MP2RAGE sequence at 7T lead to biases in cortical thickness measurements. As expected, post-hoc correction for the spatially varying $\mathrm{B}_{1}{ }^{+}$profile reduced the apparent $T_{1}$ values across the cortex in regions with low $B_{1}^{+}$, and slightly increased apparent $T_{1}$ in regions with high $\mathrm{B}_{1}^{+}$. As a result, improved contrast-to-noise ratio both at the GM-CSF and GM-WM boundaries can be observed leading to more accurate surface reconstructions and cortical thickness estimates. Overall, the changes in cortical thickness ranged between a $5 \%$ decrease to a $70 \%$ increase after $\mathrm{B}_{1}^{+}$correction, reducing the variance of cortical thickness values across the brain dramatically and increasing the comparability with normative data. More specifically, the cortical thickness estimates increased in regions characterized by a strong decrease of apparent $\mathrm{T}_{1}$ after $\mathrm{B}_{1}{ }^{+}$correction in regions with low $\mathrm{B}_{1}{ }^{+}$due to improved detection of the pial surface The current results suggest that cortical thickness can be more accurately determined using MP2RAGE data at 7T if $\mathrm{B}_{1}{ }^{+}$inhomogeneities are accounted for.

\section{Acknowledgment}

This work was supported by Maastricht University, the Netherlands Organization for Scientific Research (NWO; VIDI grant 452-11-002) and Technology Foundation STW (12724). The authors are indebted to Prof. Dr. Andrew Webb (Leiden University Medical Centre, Leiden, Netherlands) and Dr. José Marques (Donders Institute for Brain, Cognition and Behaviour, Nijmegen, Netherlands) for providing the dielectric pads and the MATLAB code to perform the post hoc $\mathrm{T}_{1}$ correction used in this study, respectively. 


\section{1. | INTRODUCTION}

Segmentation of anatomical MRI data is considered as the in vivo gold standard for parcellating the brain into gray (GM) and white matter (WM). $T_{1}$-weighted $\left(T_{1} w\right)$ images are predominantly used due to their high sensitivity to myelin concentration and, hence, high contrast between the cerebral spinal fluid (CSF, very low intensity), GM (medium intensity) and myelin-rich WM tissue (high intensity). Subsequent morphometric analyses of these tissue classes, including cortical GM thickness or subcortical GM and WM volume, allow characterization of cross-sectional differences between groups or longitudinal changes due to aging and disease. However, determination of cortical thickness based on MRI data has been criticized. The variation of myelin density across the cortical mantle, but also errors in $\mathrm{T}_{1}$ quantification or MRI acquisition biases can result in reduced contrast between GM, WM and CSF and, as a result, in inaccurate determination of cortical thickness (Han et al., 2006; Zilles and Amunts, 2015). Several software packages, e.g. FreeSurfer (Dale et al., 1999), FMRIB's software library (FSL, Smith et al. (2004)), Statistical Parametric Mapping (SPM, Ashburner (2009)) and CBS High-Res Brain Processing tools (Bazin et al., 2014), have been developed to automatically classify different tissue classes based on image-specific criteria using varying segmentation algorithms, hereby putting a large emphasis on bias field removal. In addition, many of these packages allow incorporation of complementary MRI data, such as $\mathrm{T}_{2}{ }^{(*)}$ - or proton density (PD)-weighted images, in order to improve the accuracy of the (i.e. multimodal) segmentation algorithm by identifying non-brain tissue, e.g. dura mater and blood vessels (Helms et al., 2006; Lambert et al., 2013; Viviani et al., 2017).

Although the majority of, in particular clinical, neuroimaging data is acquired at $1.5 \mathrm{~T}$ or 3 T magnetic field strengths, technical developments and increased availability have led to increased usage of ultra-high field scanners (UHF, $\geq 7 \mathrm{~T}$ ) for neuroanatomical and functional studies (as recently reviewed by De Martino et al. (2017), Marques and Norris (2017) and Ugurbil (2017), and references herein). Compared to conventional field strengths, imaging at higher fields enables acquisition of higher signal-to-noise ratio (SNR, Pohmann et al. (2016)) data and increases in various contrasts with no or little acquisition duration penalty. The increased SNR can be utilized to acquire data with higher spatial resolution, potentially leading to reduced partial volume effects (PVE) and, hence, more precise cortical GM thickness measurements. However, technical challenges remain in ensuring high image quality across the entire field-of-view. In particular, inhomogeneous radiofrequency (RF) $B_{1}$ transmit and receive profiles lead to signal intensity variations (i.e. $\mathrm{B}_{1}{ }^{+}$and $\mathrm{B}_{1}{ }^{-}$bias fields, respectively) that hamper accurate 


\section{CHAPTER 2}

classification of WM, GM and CSF and cortical GM thickness estimates (Collins et al., 2005; Van de Moortele et al., 2005; De Martino et al., 2015; Lorio et al., 2016). Especially for submillimeter acquisitions, laborious manual work is required to correct errors of the automatic segmentation, potentially introducing observer-dependent errors and biases (Fischl et al., 2004; Despotovic et al., 2015; Polimeni et al., 2017; Gulban et al., 2018). The most severe artifacts are observed towards the inferior temporal and frontal lobe regions, preventing even their manual segmentation.

Alternatively, in contrast to typically utilized weighted $\mathrm{T}_{1}$-approaches, such as the Magnetization-Prepared Rapid Acquisition Gradient Echo (MPRAGE) sequence (Mugler and Brookeman, 1990), quantitative $T_{1}$ mapping can be used to obtain homogeneous images and accurate cortical thickness measurements by minimizing the contribution of $\mathrm{B}_{1}{ }^{+}$and $\mathrm{B}_{1}{ }_{1}^{-}$variations, and $\mathrm{PD}$ and $\mathrm{T}_{2}{ }^{*}$ effects on the image intensities (Lorio et al., 2016). The inversion recovery (IR) method is considered to be the most accurate approach to determine $T_{1}$ values, as it acquires multiple time points in the longitudinal magnetization recovery curve after a 180-degree inversion. However, the main disadvantage of this method is its temporal inefficiency. Thus, alternative approaches have been proposed to reduce scanning duration and spatial distortions (which occur when the IR approach is combined with an EPI readout to decrease scanning time), while permitting accurate $T_{1}$ determination with satisfactory spatial resolution and SNR. These include, for example, spoiled gradient echo approaches, as implemented in several methods (Deoni et al., 2003; Helms et al., 2008) or variations of the MPRAGE sequence (Marques et al., 2010; Liu et al., 2011). Here, the Magnetization-Prepared 2 Rapid Acquisition Gradient Echo (MP2RAGE) sequence has recently gained popularity in higher field strengths studies (Marques et al., 2010). It combines two gradient-recalled echo (GRE) images acquired at different inversion times (i.e. a predominantly $\mathrm{T}_{1} \mathrm{w} \mathrm{GRE}_{1}$ and PDw $\mathrm{GRE}_{2}$ ) to obtain a quantitative $\mathrm{T}_{1}$ map, calculated based on the bias-free $T_{1} w$ combination image, sequence parameters and a lookup table. As in the $\mathrm{T}_{1} \mathrm{w} / \mathrm{PD} \mathrm{w}$ approach using MPRAGE, the resulting image ideally is independent of $\mathrm{B}_{1}$; $\mathrm{PD}$ and $\mathrm{T}_{2}{ }^{*}$ effects.

These proposed alternatives minimize scanning time, but also restrict the possible range of sequence parameters, possibly leading to $\mathrm{B}_{1}^{+}$-related image inhomogeneity, which needs to be accounted for. Several correction methods have been proposed to counteract the inhomogeneities of the transmit and receive bias fields. These include the use of an optimized adiabatic RF pulse, e.g. the time resampled frequency offset compensated inversion (TR-FOCl, Hurley et al. (2010)) pulse, and strategically placed dielectric pads to improve inversion efficiency in low $\mathrm{B}_{1}{ }^{+}$regions (Teeuwisse et al., 
2012). In addition, post-hoc methods, e.g. low-pass filtering, low-order fitting of the images (Ashburner and Friston, 2005) and/or computing the $T_{1} w / P D w$ ratio image to remove the PD (i.e. $\mathrm{M}_{0}$ ), $\mathrm{B}_{1}$ and $\mathrm{T}_{2}{ }^{*}$ components (Van de Moortele et al., 2009), are often used to optimize image homogeneity by removing the apparent bias field. However, even though image inhomogeneities are largely accounted for by these MRI acquisition solutions or post-processing methods, image imperfections and classification biases may still persist. In addition, the image-based bias field removal methods discussed above will not result in more correct $T_{1}$ quantification, but only improves low spatial-frequency image homogeneity. Therefore, Weiskopf et al. (2011) combined an unified segmentation-based correction of $\mathrm{T}_{1}$ maps - acquired using an 3D FLASH with variable excitation flip angles (VFA) - for residual $\mathrm{B}_{1}{ }^{+}$inhomogeneities. Alternatively, an additional $\mathrm{B}_{1}{ }^{+}$map can be acquired to post-hoc improve $\mathrm{B}_{1}{ }^{+}$independence, as reviewed by Lutti et al. (2010) and Pohmann and Scheffler (2013), which is able to cover a broader range of $T_{1}$ mapping sequences. For the MP2RAGE sequence, the use of different (low) flip angles for the GRE images $\left(a_{1}\right.$ and $\left.a_{2}\right)$ renders the MP2RAGE T, map largely, but not entirely, free from $\mathrm{B}_{1}{ }^{+}$effects. Here, correction using the Saturation-prepared with 2 rapid Gradient Echoes (Sa2RAGE) sequence has been used to remove residual $\mathrm{B}_{1}{ }^{+}$effects (Eggenschwiler et al., 2012; Marques and Gruetter, 2013).

Initial work has shown that post-hoc $\mathrm{B}_{1}{ }^{+}$correction of MP2RAGE maps improves the subcortical GM vs. WM contrast-to-noise ratio (CNR), facilitating automatic segmentation (Marques and Gruetter, 2013). However, the effect of this correction scheme has not yet been extensively characterized across cortical regions. Based on the initial results by Marques et al. (2017) and the $\mathrm{B}_{1}{ }^{+}$correction methodology, the difference in the degree of $T_{1}(W)$ signal changes depends on the apparent $T_{1}$ value and, therefore, vary between WM and GM, even if the voxels are very close to each other. As such, the correction also affect the CNR between cortical GM and WM, and we expect this to propagate towards differences in the cortical surface reconstructions and, therefore, apparent cortical thickness. Thus, the aim of the current study is to quantify the changes of cortical thickness, as well as the underlying changes in estimated $\mathrm{T}_{1}{ }^{1}$, after applying the proposed $\mathrm{B}_{1}{ }^{+}$correction method. To quantify the effect of removing residual $\mathrm{B}_{1}{ }^{+}$bias, the longitudinal analysis stream within the newly released FreeSurfer v6.0 was used (Reuter and Fischl, 2011). This allows high-resolution $\left(<1 \mathrm{~mm}^{3}\right)$ surface reconstructions of the $\mathrm{B}_{1}{ }^{+}$uncorrected and corrected MP2RAGE data, which are conseaffected by $B_{1}^{+}$correction. Therefore, when referring to changes in calculated $T$, values throughout the manuscript, it should read "changes in apparent/measured $T_{1}^{\text {" }}$ and not "changes in intrinsic $T_{1}$ ". 


\section{CHAPTER 2}

quently directly comparable due to matching topology and number of vertices, using a surface-based analysis approach similar as in Fujimoto et al. (2014). Finally, subsequent regional cortical thickness averages are compared to normative data based on the model presented in Potvin et al. (2017) to evaluate their accuracy. The model provides subject-specific cortical thickness estimates for cortical regions based on the subject's demographics and scanner characteristics.

\section{2. | MATERIALS \& METHODS}

\subsubsection{Subjects and data acquisition}

Sixteen healthy volunteers (age $=39 \pm 13.8$, between 20 and 66 years old, 4 males) were included in this study after providing written informed consent in accordance with the Declaration of Helsinki. The protocol was approved by the ethics review board of the Faculty of Psychology and Neuroscience, Maastricht University, the Netherlands. MRI data were acquired using a whole-body 7T magnet (Siemens Healthineers, Erlangen, Germany) and a 32-channel phased-array head coil (Nova Medical, Wilmington, MA, USA). High resolution ( $0.7 \mathrm{~mm}$ isotropic nominal voxel size) whole-brain quantitative $T_{1}$ images were obtained with the MP2RAGE (Marques et al., 2010) sequence, and the SA2RAGE (Eggenschwiler et al., 2012) sequence was used to map $B_{1}^{+}(2 \mathrm{~mm}$ isotropic nominal voxel size) across the brain. MP2RAGE data were acquired with TR/ $\mathrm{TE}=5000 / 2.47 \mathrm{~ms}, \mathrm{TI}_{1} / \mathrm{TI}_{2}=900 / 2750 \mathrm{~ms}, \mathrm{a}_{1} / \mathrm{a}_{2}=5^{\circ} / 3^{\circ}$ and generalized autocalibrating partially parallel acquisitions (GRAPPA) factor $=3$ in the phase-encoding (PE) direction (anterior-posterior) with 24 references lines. For the SA2RAGE, the parameters were: $\mathrm{TR} / \mathrm{TE}=2400 / 0.78 \mathrm{~ms}, \mathrm{TD}_{1} / \mathrm{TD}_{2}=58 / 1800 \mathrm{~ms}, \mathrm{a}_{1} / \mathrm{a}_{2}=4^{\circ} / 11^{\circ}$ and GRAPPA factor $=2 \mathrm{in}$ PE direction (anterior-posterior) with 24 references lines. See Haast et al. (2016) for further details on other scanning parameters. The TR-FOCI inversion pulse (Hurley et al., 2010) and dielectric pads containing a $25 \%$ suspension of barium titanate in deuterated water, placed around the head proximal to the temporal lobe areas (Teeuwisse et al., 2012), were used to improve $\mathrm{B}_{1}^{+}$homogeneity across the brain and locally, respectively.

\subsection{2. | Pre-processing pipeline}

Several pre-processing steps were performed to improve subsequent automatic segmentation. First, the data were skull-stripped by using the different MP2RAGE output volumes (UNI, T map and INV2; see Marques et al. (2010)). The INV2 (i.e. PDw) image was used to obtain a brain mask, as it provides the best intra- and extra-cranial tissue contrast, especially after removal of any RF bias field using ANTs' (Advanced Normal- 
ization Tools) N4BiasFieldRemoval tool (Tustison et al., 2010). Remaining non-brain tissue was removed using probability maps of the dura mater and arteries. These initial steps were performed using MIPAV 7.1.1 (Center for Information Technology, NIH, Bethesda, MD, USA), JIST 3.0 (Johns Hopkins University, Baltimore, MD, USA) and CBS High-Res Brain Processing tools 3.0.9 (Max Planck Institute for Human Cognitive and Brain Sciences, Leipzig, Germany). Both the MP2RAGE UNI and $T_{1}$ images were post-hoc-corrected for variations in $\mathrm{B}_{1}^{+}$using the same method, as described in Marques and Gruetter (2013) ${ }^{2}$. Briefly, the 'original'MP2RAGE UNI volume and Sa2RAGE $\mathrm{B}_{1}{ }^{+}$map were used to generate the 'corrected' UNI image and $\mathrm{T}_{1}$ map. Given the higher independence of $\mathrm{B}_{1}{ }^{+}$estimation on the $\mathrm{T}_{1}$ values, the $\mathrm{B}_{1}{ }^{+}$map was first optimized for the varying $T_{1}$ across the brain using $2 D$ interpolation and the Sa2RAGE lookup table. This newly generated $\mathrm{B}_{1}{ }^{+}$map was consecutively used to generate a $T_{1}$ map by taking into account the varying $\mathrm{B}_{1}{ }^{+}$across the brain using $2 \mathrm{D}$ interpolation and the MP2RAGE lookup table. This process was repeated three times and, as in the original paper, variations in both $\mathrm{B}_{1}^{+}$and $\mathrm{T}_{1}$ were found to be under $10^{-3}$ for the last iteration. The $\mathrm{B}_{1}^{+}$ dependency plot of the $T_{1}$ map for the current sequence parameters is displayed in Fig. 2.1.

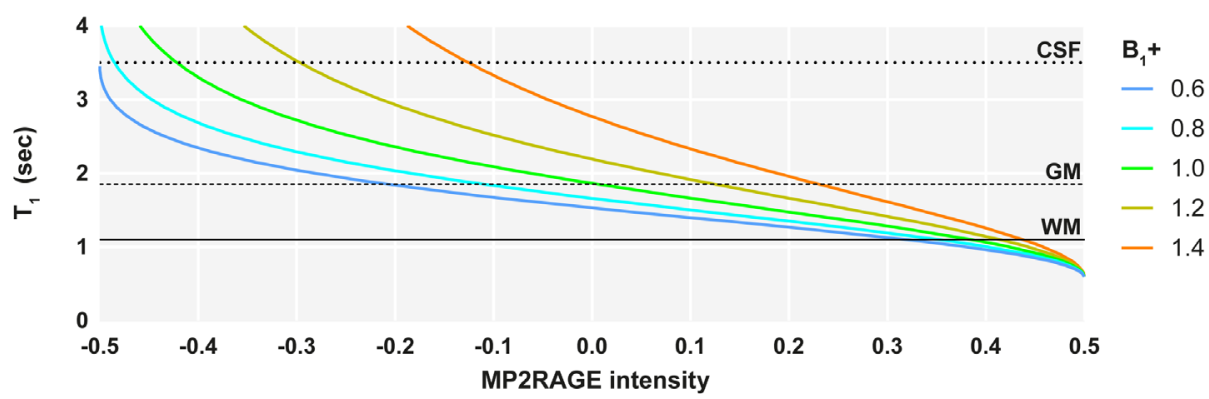

Fig. 2.1. $\mid$ MP2RAGE $B_{1}{ }^{+}$dependency. $B_{1}{ }^{+}$dependency of the $T_{1}$ map for a range of $B_{1}{ }^{+}$values (colored solid lines). Typical WM, GM, and CSF T, values are indicated using the vertical lines.

Noteworthy, by computing the $\mathrm{GRE}_{\mathrm{T} 11}$ and $\mathrm{GRE}_{\mathrm{T} 12}$ ratio (i.e. $\mathrm{T}_{1} \mathrm{w}$ ) image, high background ('salt and pepper') noise outside of the brain is introduced, in addition to increased noise in the meninges (O'Brien et al., 2013). These can be masked out, but differences in the noise- and $\mathrm{T}_{1}$-dependent $\mathrm{CNR}$, induced after the $\mathrm{B}_{1}{ }^{+}$correction step, can also affect the performance of the skull-stripping algorithms. In particular, in thin cortical regions (e.g. near the occipital cortex), the GM is not easily separable from other structures, such as the dura mater and cerebral sinuses, because of the comparable image intensity. In order to eliminate any additional methodological bias related to this and

2 The code used to perform the $B_{1}^{+}$correction is publicly available at: https://github.com/ JosePMarques/MP2RAGE-related-scripts. 


\section{CHAPTER 2}

to tie the differences purely to changes in $T_{1}$ and CNR, the same subject-specific binary brain mask was used for both datasets. Gradient non-linearity correction without jacobian intensity correction (to preserve the quantitative $T_{1}$ values) was applied on the brain extracted $\mathrm{B}_{1}^{+}$corrected MP2RAGE UNI and other (including the original) volumes using the gradient coefficients file provided by the scanner manufacturer and the Human Connectome (HCP) high-res analysis 'gradunwarp' tool (https://github. com/Washington-University/gradunwarp). Finally, the skull-stripped and gradient distortion unwarped MP2RAGE UNI volumes were aligned using affine transformation to the $\mathrm{MNI}$ space $(0.7 \mathrm{~mm})$ template prior to cortical surface reconstruction.

\subsection{3. | Surface reconstruction pipeline}

High-resolution cortical reconstruction was performed with the longitudinal processing stream implemented in the FreeSurfer (v6.0, http://surfer.nmr.mgh.harvard. edu/) image analysis suite (Dale et al., 1999; Reuter et al., 2012). This was necessary to enable direct (i.e. vertex-by-vertex) comparison between the surface reconstructions and cortical thickness surface metric based on either the original or $\mathrm{B}_{1}{ }^{+}$corrected MP2RAGE UNI images. First, a template volume was computed using both images to obtain initial white matter (WM-GM boundary) and pial (GM-CSF) surfaces (Reuter and Fischl, 2011). These surfaces were then aligned with the original and corrected volumes, whose intensity values were subsequently used to deform surfaces following the same procedure as in the standard FreeSurfer processing stream. Please note that computation of the template surfaces is crucial to obtain a 'bias-free' starting point for generating surfaces optimized for either the original or the corrected volumes. This resulted in two different datasets, but with matching mesh topology and the same number of vertices. No manual corrections were performed in order to avoid bias towards one of the datasets.

\subsection{4. | Post-processing pipeline}

For each subject, the WM and pial surfaces (derived from the corrected MP2RAGE dataset) and associated cortical thickness map were used to project the (original and corrected) $\mathrm{T}_{1}$ and $\mathrm{B}_{1}{ }^{+}$maps onto the surface. This was done using FreeSurfer's mri vol2surf function and by averaging between 20 and $80 \%$ of the cortical thickness (with steps of $0.05 \%$ ) to reduce partial voluming with WM and CSF. Subsequently, $T_{1}$ and cortical thickness difference maps were calculated by subtracting the values based on the original maps from that of the corrected maps. In addition, for each vertex and (white and pial) surface, the change in the vertex's location (in $\mathrm{mm}$, along the vertex's normal) for the corrected (vs. original) volume was computed and projected onto the 
surface (Fujimoto et al., 2014). All surface maps were coregistered to the 'fsaverage' subject using sphere-based alignment (Fischl et al., 1999) for further (statistical) analyses. Final surface maps were visualized using the Connectome Workbench v1.2.3 viewer (Washington University School of Medicine, Saint Louis, MO, USA) after conversion of the inflated surfaces and overlays to a compatible format. Non-cortical vertices in between hemispheres were masked out using FreeSurfer's parcellation scheme and excluded from the comparisons. In addition, region-wise comparisons of average $\mathrm{T}_{1}$ (including inter-regional coefficient of variation) and cortical thickness were performed following the parcellation provided by FreeSurfer (i.e. 'Desikan-Killiany Atlas', Desikan et al. (2006)). Finally, to test the accuracy of the cortical thickness measures, normative cortical thickness data were calculated using the model presented in Potvin et al. (2017). For each subject, estimates of their expected regional cortical thickness based on age, gender and estimated total intracranial volume and the scanner characteristics were obtained. In the current study, scanner manufacturer was set to 'Siemens' and magnetic field strength to ' $3 T$ ', the highest possible. This resulted in a set of normative cortical thickness data, which can account for different ages and gender. MATLAB (R2015B, The MathWorks, Natick, Massachusetts, USA) was used to compute the Euclidean distance, $\mathrm{d}$, between the two vectors (i.e. $\vec{u}$, normative data and $\vec{v}$, cortical thickness based on original or corrected data) across all subjects, $n$, for each region:

$$
d(\vec{u}, \vec{v})=\|\vec{u}-\vec{v}\|=\sqrt{\left(u_{1}-v_{1}\right)^{2}+\left(u_{2}-v_{2}\right)^{2} \ldots\left(v_{n}-v_{n}\right)^{2}}
$$

For each region, the difference in Euclidean distance (i.e. $d_{\text {diff }}=d_{\text {original }}-d_{\text {corrected }}$ ) was mapped onto an inflated surface to indicate whether the average cortical thickness became more comparable (i.e. $d_{\text {diff }}>0$ in red/yellow) or less comparable (i.e. $d_{\text {diff }}<0$ in blue/green) to the normative data after the $\mathrm{B}_{1}{ }^{+}$correction.

\subsection{5. | Statistical analyses}

Whole-brain vertex-wise analyses using FreeSurfer's QDEC tool were performed to detect vertices characterized by a significant (false discovery rate (FDR)-corrected) change in $\mathrm{T}_{1}$ and/or cortical thickness after $\mathrm{B}_{1}{ }^{+}$correction. In addition, pairwise $t$-tests and MATLAB were used to test for statistical differences in average $T_{1}$ or cortical thickness across the entire GM or per region, between the original and corrected data. Finally, correlation coefficients between original or corrected and normative cortical thickness data were calculated. 


\section{CHAPTER 2}

\section{3. | RESULTS}

Fig. 2.2A shows cross-sectional (axial) images from the original (first column) and corrected (second) MP2RAGE $T_{1}$ maps, corresponding corrected-original $T_{1}$ difference (third) and $\mathrm{B}_{1}^{+}$map (last) from a single-subject, for illustrative purposes. Most pronounced $\mathrm{T}_{1}$ changes (mostly decrease, in green) were mainly observed along the cortex and clearly reflected the differences of $\mathrm{B}_{1}^{+}$across the brain based on the correspondence with the $\mathrm{B}_{1}^{+}$map. This was confirmed by the clear change of the GM (solid lines) and WM (dashed) $T_{1}$ distributions (averaged across subjects after normalization for the number of voxels) obtained from the $\mathrm{B}_{1}^{+}$-corrected (blue) versus that of the original (red) $T_{1}$ maps (see Fig. 2.2B). It is apparent that the variance and the mean of the $T_{1}$ values reduce for $G M$ and less for $W M$ after taking $B_{1}{ }^{+}$into account. As such, the area of the overlap between the GM and WM histograms for the entire brain increased
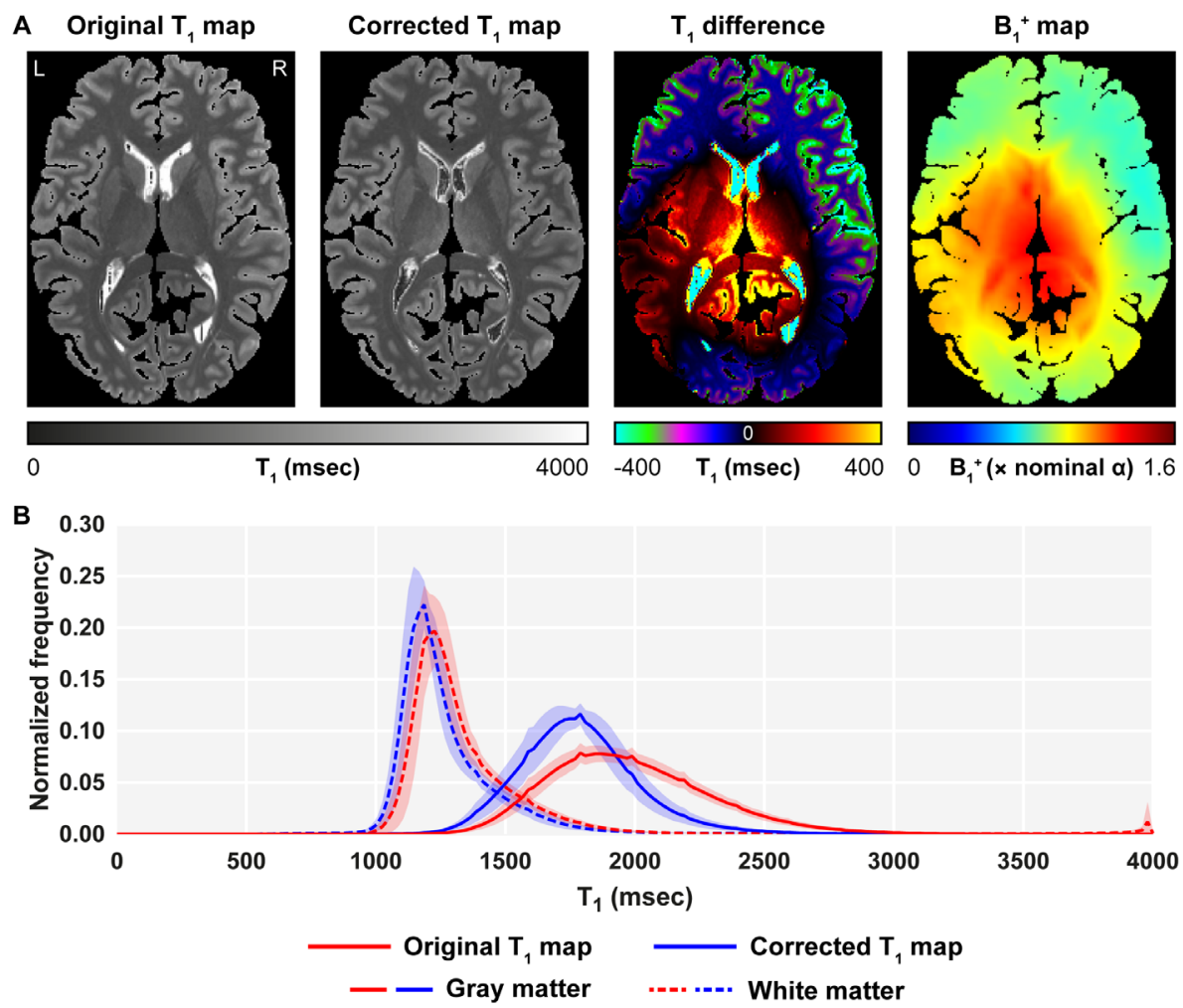

Fig. 2.2. | MP2RAGE and Sa2RAGE volume data. Example original and corrected MP2RAGE T, maps (msec), the difference between them ( $m s e c)$ and corresponding $B_{1}{ }_{1}$ map ( $\times$ nominal value) are shown (left to right, B). Normalized (for number of voxels) gray (solid lines) and white matter (dashed) $T_{1}$ distributions (averaged across subjects, \pm S.D.) are shown for both the original (red lines) and corrected (blue) $T_{1}$ maps (B). Here, the same tissue masks (based on the corrected data) were used to compute the $T_{1}$ histograms for the original and corrected data. 
after the $\mathrm{B}_{1}{ }^{+}$correction $\left(0.534 \pm 0.07\right.$ vs. $0.592 \pm 0.06$, paired t-test, $\mathrm{t}_{15}=10.59, \mathrm{p}<$ 0.0001). However, when only the temporal lobe is considered (histograms not shown), this significantly decreased $\left(0.435 \pm 0.08\right.$ vs. $0.372 \pm 0.09$, paired t-test, $\mathrm{t}_{15}=8.81, \mathrm{p}<$ 0.0001 ), indicating improved separability of GM and WM. No significant difference was observed for the cingulate cortex $(0.265 \pm 0.07$ vs. $0.262 \pm 0.08)$.
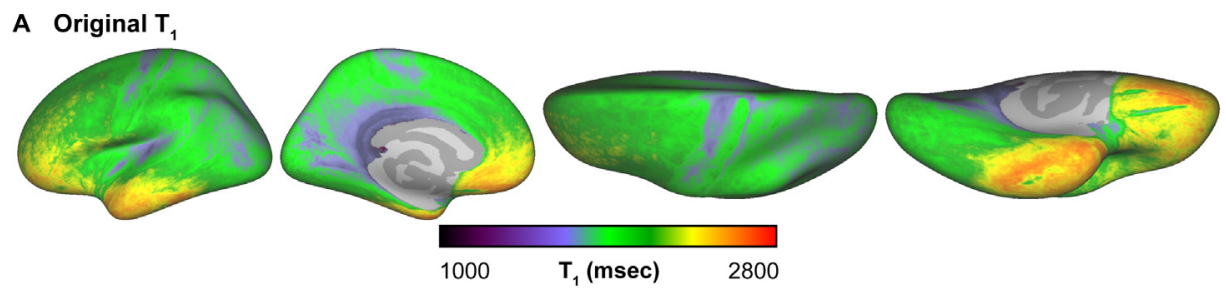

B Corrected $\mathrm{T}_{1}$
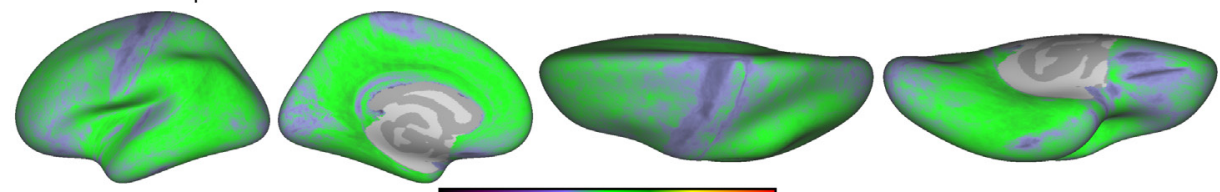

C $T_{1}$ difference
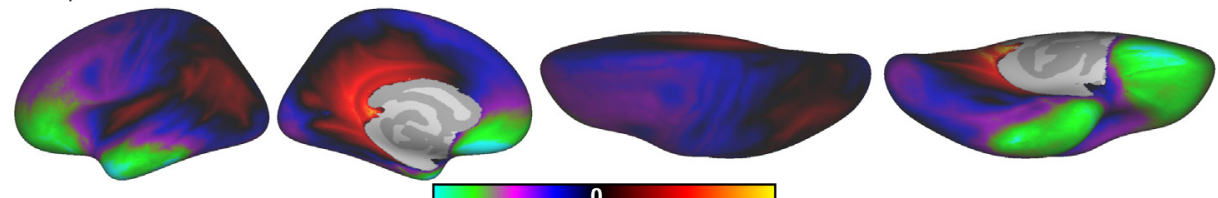

D Statistical difference
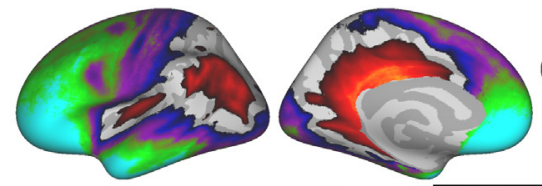

Decrease

$-30 \quad T_{1}$ difference (\%) 30

\section{0}




\section{CHAPTER 2}

In order to better appreciate the distribution of the observed changes, surface representations of the original and corrected $T_{1}$ maps are shown in Fig. 2.3A and $B$ for the left hemisphere. As very similar observations are made for the right hemisphere, the respective data are shown in Supplementary Fig. 2.1. Note that the same scaling for both original (mean surface-based $\mathrm{GM} \mathrm{T}_{1}=1944.6 \pm 223.1 \mathrm{~ms}$ ) and corrected $\mathrm{T}_{1}$ $\left(1794.3 \pm 124.8 \mathrm{~ms}\right.$, paired t-test, $\left.\mathrm{t}_{15}=7.966, \mathrm{p}<0.0001\right)$ maps was applied to be able to directly compare $T_{1}$ values based on the color scheme. Here, a gradient towards higher values (e.g. from green to yellow/red) in more inferior parts of the brain was observed in the original data, which mirrors the observed variation of $\mathrm{B}_{1}^{+}$across the cortex (see Fig. 2.3E). This gradient was not visible in the corrected $\mathrm{T}_{1}$ maps. After the $\mathrm{B}_{1}{ }^{+}$correction, $T_{1}$ most significantly (vertex-wise comparison, FDR-corrected $p<0.05$ ) decreased in the inferior affected areas (e.g. inferior temporal and frontal lobes), but increased (although to a smaller extent) in the posterior cingulate cortices and left parietal lobe (the latter for left hemisphere only) compared to the original $\mathrm{T}_{1}$ maps (see Figs. 2.3C and 3D). On average, cortical $T_{1}$ was changed -150.3 ( \pm 73.1$)$ ms after the correction.

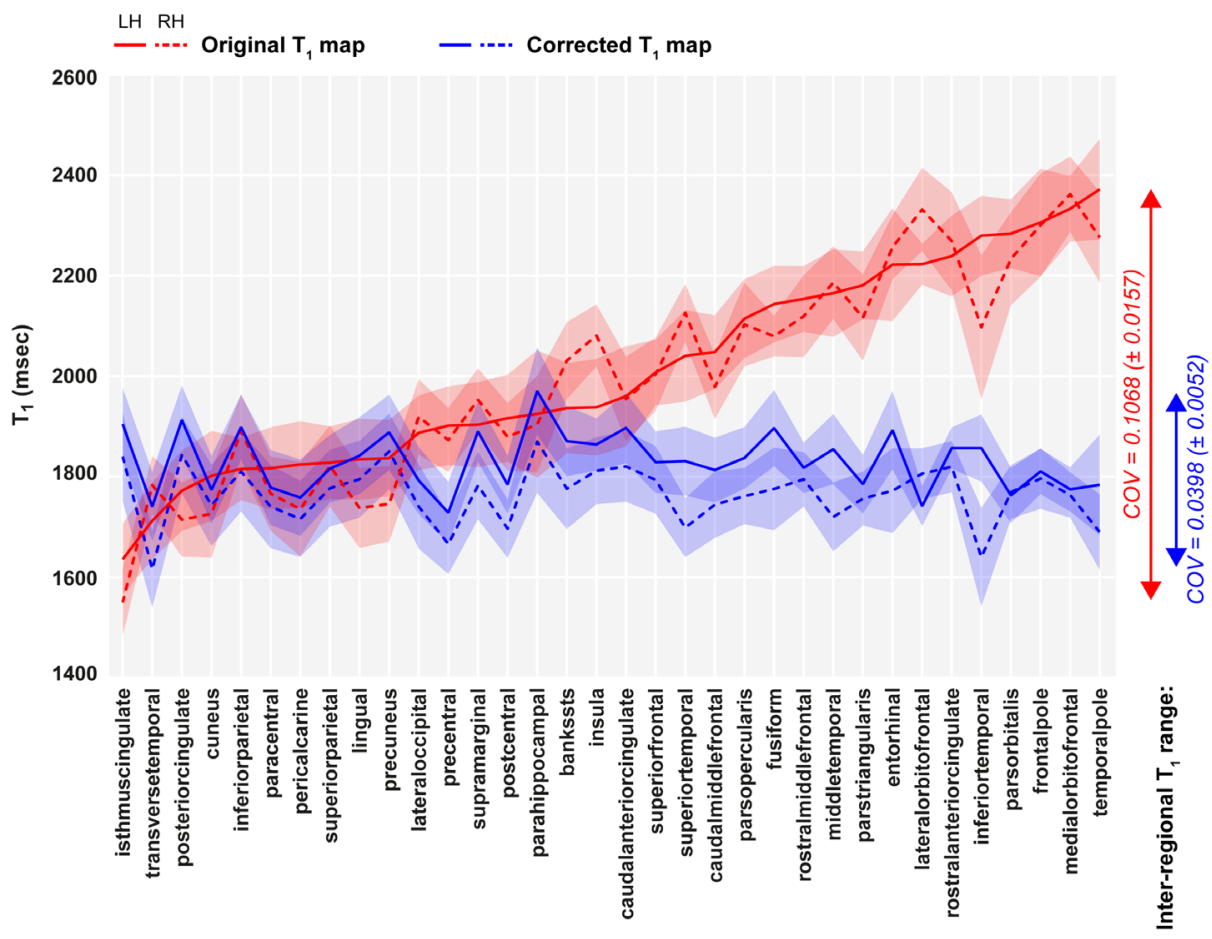

Fig. 2.4. | Regional $T_{1}$ averages before and after $B_{1}{ }^{+}$correction. Original (red lines) and corrected (blue) across subjects $T_{1}$ averages ( $y$-axis, $m s e c \pm S . D$ ) are plotted across all regions ( $x$-axis) for both the left (solid lines) and right (dashed) hemispheres. Regions are sorted based on the original $T_{1}$ map. Vertical lines to the right of the graph indicate the region-wide range of $T_{1}$ values and corresponding inter-regional coefficient of variation. 
Region-wise $T_{1}$ averages (across subjects \pm S.D.) are shown in Fig. 2.4. Regions (x-axis) were sorted based on the original $\mathrm{T}_{1}$ (red lines) values ( $\mathrm{y}$-axis) on the left hemisphere (solid). Vertical lines to the right of the graph indicate the observed range of $\mathrm{T}_{1}$ values across regions. On average, the original regional $\mathrm{T}_{1}$ values are within a $904.3 \mathrm{~ms}$ range (from $1521.6 \mathrm{~ms}$ to $2425.9 \mathrm{~ms}$ ), but this was significantly reduced to $430.2 \mathrm{~ms}$ (1597.4 to $2027.6 \mathrm{~ms}$, paired t-test, $\left.\mathrm{t}_{15}=13.69, \mathrm{p}<0.0001\right)$ for the corrected data. This resulted in an inter-regional COV of $0.107( \pm 0.016)$ versus $0.040( \pm 0.005)$, respectively. In particular, regions with high $\mathrm{T}_{1}$ values were affected by the $\mathrm{B}_{1}{ }^{+}$correction. Largest region-wise differences in $T_{1}$ between the original and corrected data were observed for the temporal and frontal poles, as well as the medial orbitofrontal sulci and inferior temporal gyri.
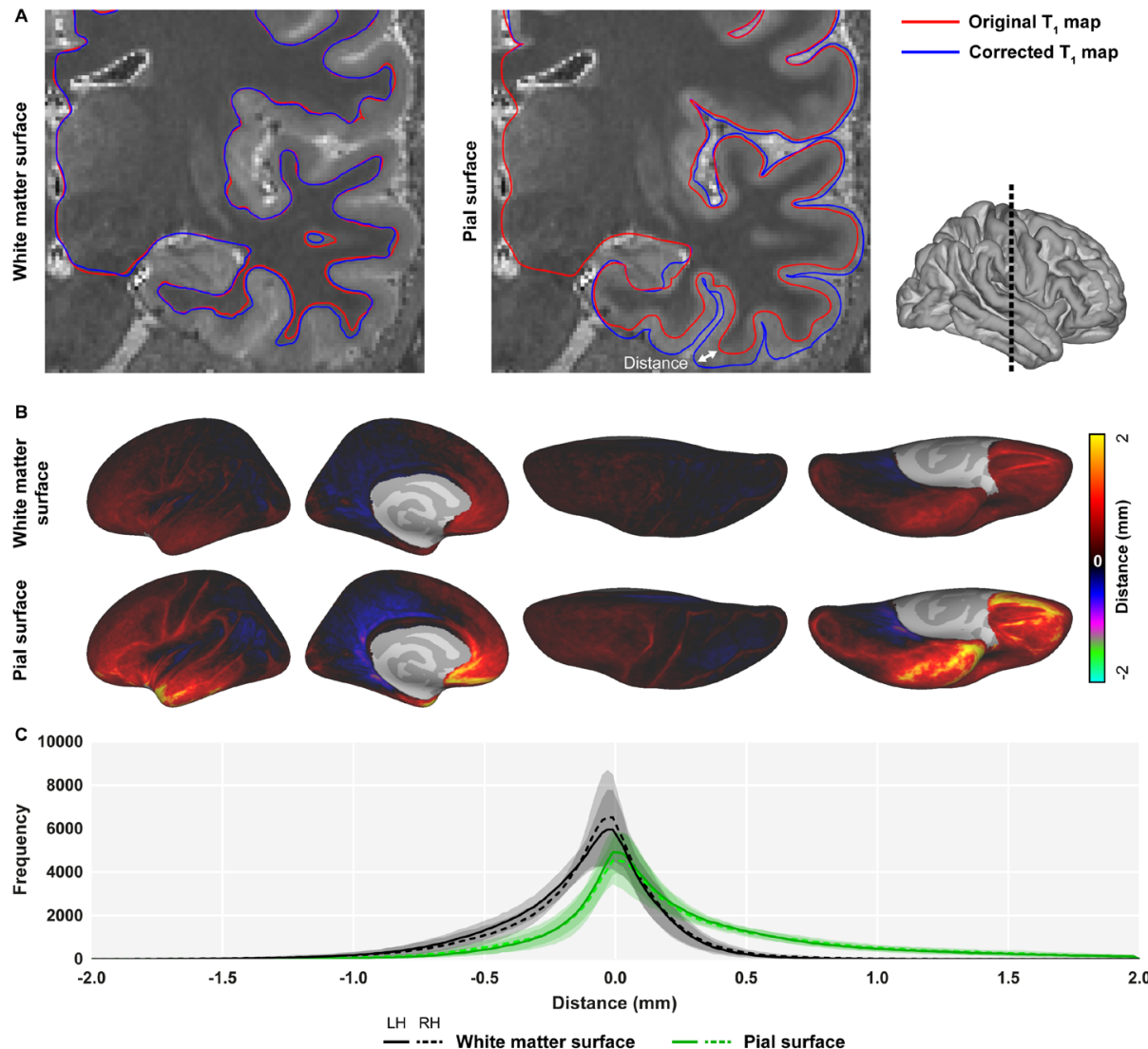

Fig. 2.5. | White matter and pial surface reconstructions. Example white matter (left) and pial (right) surface reconstructions based on the original (red lines) and corrected (blue) $T_{1}$ maps are shown (A). The spatial difference $(\mathrm{mm})$ between both surfaces (white matter: top and pial: bottom), based on the original and corrected data, are mapped onto an inflated left hemisphere surface (B). Average distributions (across subjects \pm S.D.) of these differences are plotted for both white matter (black) and pial (green) surfaces, and left (solid lines) and right (dashed) hemispheres. 


\section{CHAPTER 2}

To illustrate the effect of the $\mathrm{B}_{1}{ }^{+}$correction on the cortical segmentation, both sets (original, in red, and corrected, in blue) of white matter (left column) and pial (right) surfaces were overlaid onto a coronal slice (of the temporal lobe at the location of the dashed line) of the $\mathrm{B}_{1}^{+}$-corrected MP2RAGE $\mathrm{T}_{1}$ map (see Fig. 2.5A for a single-subject example, and Supplementary Fig. 2.2 for additional examples from multiple subjects). The distance (in mm, e.g. indicated by white double arrow) along the vertex's normal between original and corrected surfaces was then mapped onto the surface for the left hemisphere (see Fig. 2.5B and Supplementary Fig. 2.3 for right hemisphere), so that negative values indicated inwards movement (i.e. towards the center of the brain) while positive values indicated outwards movement of the surface for the corrected data. While both the (left and right) WM and pial surfaces followed the same pattern,

\section{A Original cortical thickness}
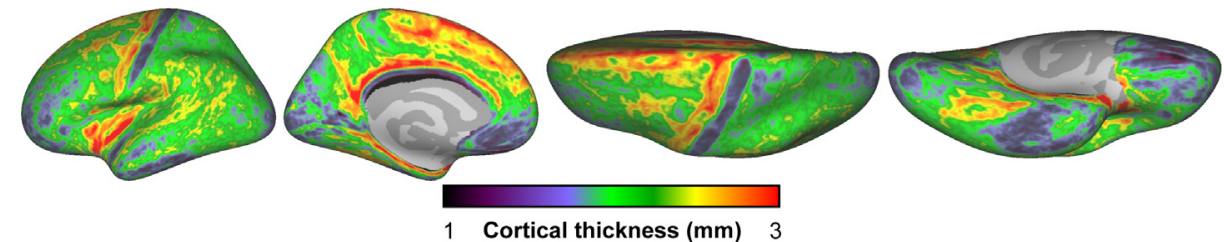

B Corrected cortical thickness
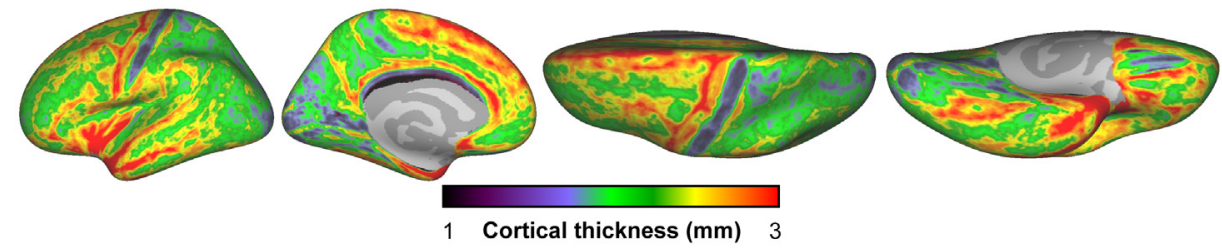

C Cortical thickness difference
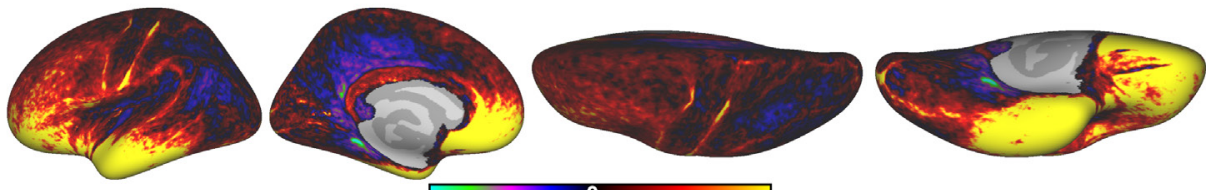

D Statistical difference

-30 Cortical thickness 30
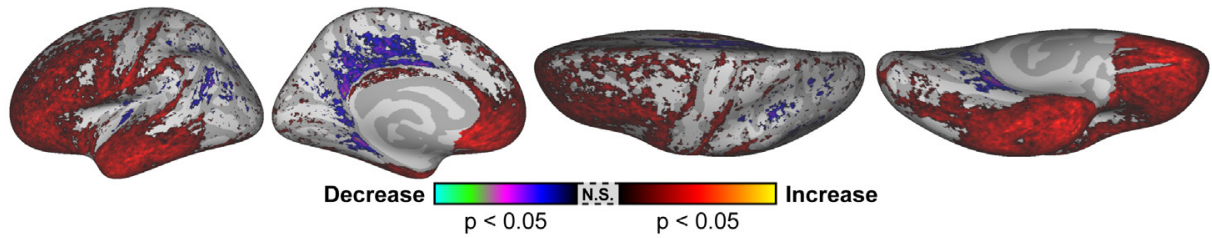

Fig. 2.6. |Average cortical thickness surface maps. Original (A) and corrected (B) cortical thickness $(\mathrm{mm})$, cortical thickness difference $(\%, C)$, and statistical difference (D) were mapped onto an inflated left hemisphere surface and averaged across all subjects. Only vertices with a significant (FDR-corrected $p<0.05$ ) decrease (blue/green) or increase (red/yellow) of cortical thickness are highlighted in $D$ and grey vertices are non-significant (N.S.). 
a stronger effect was observed for the pial surface, for which, especially in the inferior frontal and temporal lobes, the surface expanded (shown in red/yellow). On the other hand, both WM and pial surfaces were placed more inwards after correction (shown in blue) in the vicinity of the posterior cingulate cortices. On average, the WM and pial surfaces were moved by -0.104 ( \pm 0.054, see black histograms in Fig. $2.5 C$ ) and 0.255 ( \pm 0.092 , green) $\mathrm{mm}$, and the corresponding distributions showed a slight bias towards negative and positive values, respectively.

Figs. 2.6A and B show the resulting cortical thickness maps computed from the surfaces derived from the original or corrected data, respectively. Mean cortical thickness (across the entire ribbon) for the original data was significantly lower $(2.117 \pm 0.053 \mathrm{~mm})$ than the cortical thickness based on the corrected data $\left(2.282 \pm 0.06 \mathrm{~mm}\right.$, paired t-test, $\mathrm{t}_{15}=$ 11.69, $\mathrm{p}<0.0001$ ). Most significant differences in cortical thickness (see Fig. 2.6C) were observed in the inferior frontal and temporal lobes (vertex-wise comparison, FDR-corrected $p<0.05$, see Fig. 2.6D). Again, comparable differences were observed across hemispheres, except the decreased cortical thickness in the parietal lobe did not reach the significance level for the right hemisphere (see Supplementary Fig. 2.4).

In addition, left and right hemisphere average (across subjects) cortical region $\mathrm{T}_{1}$ and thickness changes (in \%) were inversely correlated (Pearson correlation, $r_{33}=$ -0.870, $p<0.001$ and $r_{33}=-0.839$, $p<0.001$, respectively). That is, a $\mathrm{T}_{1}$ decrease after $\mathrm{B}_{1}{ }^{+}$correction led to an increase in measured cortical thickness (see Fig. 2.7 and Supplementary Fig. 2.5).

Fig. 2.7. |Regional $T_{1}$ and cortical thickness changes. Across subjects average $T_{1}$ (x-axis) and cortical thickness (y-axis) changes (\%, \pm S.D.) are plotted for each region (black dots) for the left hemisphere. Dashed red lines represent the best fit $\pm 95 \% \mathrm{Cl}$. Boldface $p$-values indicate a significant correlation.

\section{- Cortical region ----- Linear fit $( \pm 95 \% \mathrm{Cls})$}

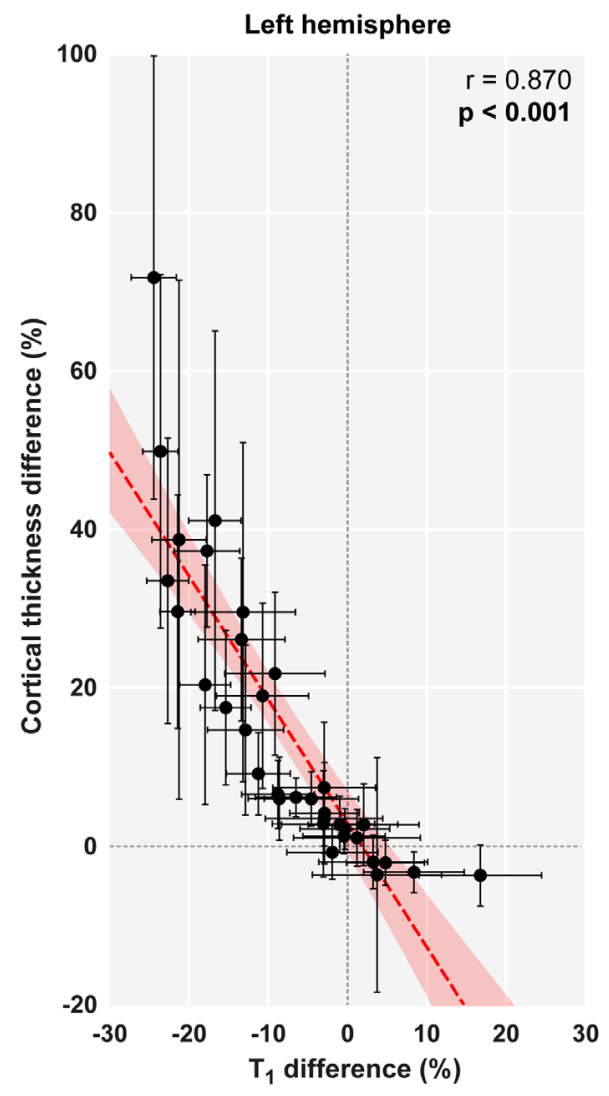




\section{CHAPTER 2}

Finally, in order to benchmark the accuracy of the cortical thickness measurements, regional cortical thickness data were compared to the normative data calculated based on the demographics of the study population (see green dots in Supplementary Fig. 2.6A). Please note that normative could not be computed for the banks of superior temporal sulcus (i.e. 'bankssts'), frontal and temporal poles. In general, more close agreement with the normative values (green filled circles) was reached for the corrected data (blue bars) compared to the original data (red bars), especially in the regions where the largest cortical thickness changes were observed (from bottom and

A

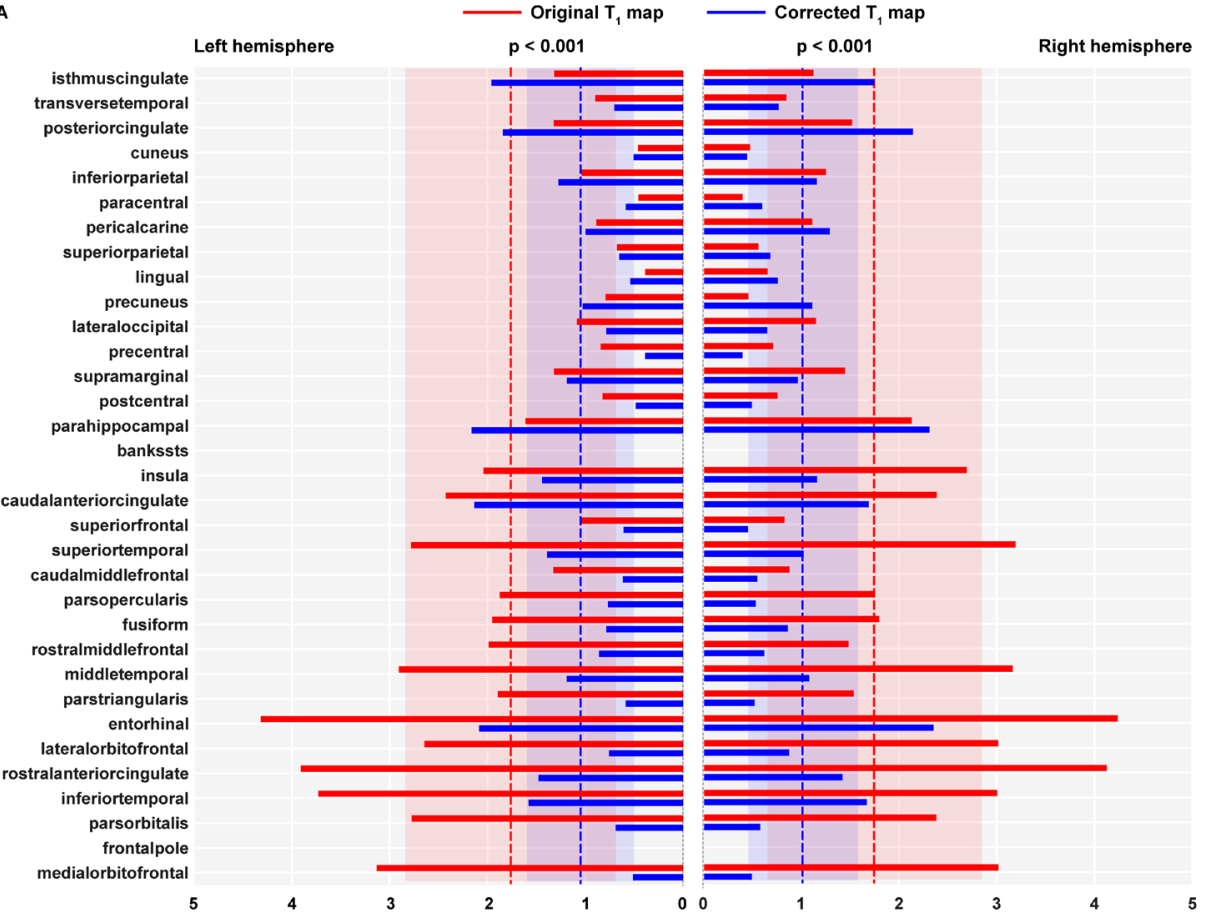

B

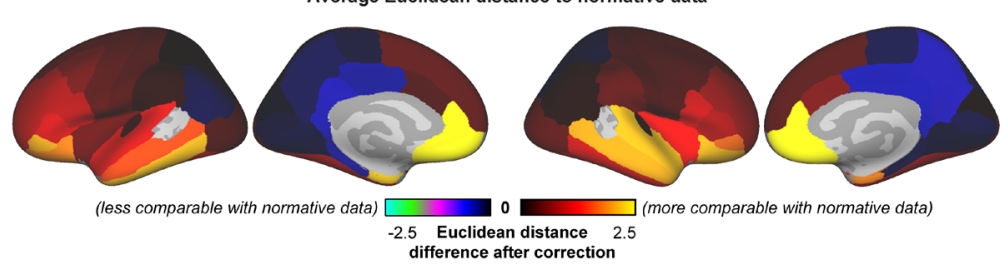

Fig. 2.8. | Comparison between regional cortical thickness averages with normative data. Euclidean distances between original (red bars) and corrected (blue) cortical thickness averages ( $x$-axis) and normative data are plotted across all regions (y-axis) for both the left (left column) and right (right) hemispheres. Vertical dashed lines indicate the average Euclidean distance across all regions for original (red) and corrected (blue) $T_{1}$ maps, while p-values on top of the graph indicate the significance level after pair-wise comparison across regions. Regions are sorted based on the original $T_{1}$ map (see Fig. 2.3). 
up). As such, the correlation between the measured and normative cortical thickness values increased after the $\mathrm{B}_{1}{ }^{+}$correction (see Supplementary Fig. 2.6B). For each region, the Euclidean distance (across all subjects) between each dataset and the normative dataset was calculated to quantify comparability (see Fig. 2.8A). The Euclidean distance was significantly lower for the corrected data compared to the original data for both the left $\left(1.049 \pm 0.545\right.$ vs. $1.761 \pm 1.073$, paired t-test, $\left.t_{30}=4.126, p<0.001\right)$ and right $\left(1.019 \pm 0.566\right.$ vs. $1.750 \pm 1.100$, paired t-test, $\left.t_{30}=4.177, p<0.001\right)$ hemispheres. The regional quantitative differences were then mapped onto the surface (see Fig. 2.8B) to compare with the changes in $\mathrm{T}_{1}$ and $\mathrm{B}_{1}{ }^{+}$(see Figs. 2.3B and $\mathrm{E}$ ). Here, red/yellow indicates higher comparability while blue/green indicates lower comparability with the normative data. Strongest changes in comparability were observed for regions characterized by pronounced $\mathrm{T}_{1}$ changes and deviations of $\mathrm{B}_{1}{ }^{+}$. Here, the comparability increased for the temporal lobes, but decreased for the anterior cingulate cortex.

\section{4. | DISCUSSION}

Despite tremendous improvements, UHF data still suffers from image imperfections related - among other sources - to $B_{1}$ inhomogeneities, leading to various errors in MRI-based cortical thickness measurements and/or relaxometry (Collins et al., 1998; Vaughan et al., 2001; Padormo et al., 2016; Marques and Norris, 2017; Ugurbil, 2017). In particular, quantitative $\mathrm{T}_{1}$ mapping approaches ideally allow acquisition of unbiased anatomical data with any influence of scanner imperfections removed, in contrast to conventional $\mathrm{T}_{1} \mathrm{w}$ images. However, residual $\mathrm{B}_{1}{ }^{+}$-related imperfections may still persist for many standard quantitative acquisition schemes.

In the current study, we investigated the effect of residual $\mathrm{B}_{1}{ }^{+}$inhomogeneities on $\mathrm{T}_{1}$ and cortical thickness estimates based on $T_{1}(w)$ MP2RAGE data. Recently, the MP2RAGE sequence gained popularity at UHF, due to its easy implementation and efficiency to map $T_{1}$ at a submillimeter scale without significantly sacrificing SNR (and CNR). The MP2RAGE sequence allows the acquisition of a quantitative $T_{1}$ map and $T_{1} w$ image within a reasonable (i.e. $<10 \mathrm{~min}$ ) time frame (Marques et al., 2010). Whereas the $\mathrm{T}_{1}$ map can be directly used to quantify microstructural (e.g. myelin)-related changes of the brain (Stuber et al., 2014), the $T_{1}$ w image can be readily processed by publicly available software, such as the widely used FreeSurfer, to obtain cortical thickness maps (Fischl and Dale, 2000). However, it has been noted that the quantitative $T_{1}$ values using the MP2RAGE sequence, similar to other quantitative $T_{1}$ approaches, are affected by $B_{1}$ - and $\mathrm{B}_{1}{ }^{+}$imperfections. In order to reduce the sensitivity to image inhomogeneities, particularly prominent at UHF, we utilized an adiabatic TR-FOCI inversion pulse together with 


\section{CHAPTER 2}

dielectric pads proximal to the temporal lobes (O'Brien et al., 2014). Nevertheless, the persisting spatially varying $\mathrm{B}_{1}{ }^{+}$field may still lead to an erroneous estimate of $\mathrm{T}_{1}$ due to deviation of the excitation flip angle from its nominal value, leading to decreased intra-subject reproducibility, and increased across subjects and studies variation of GM and $\mathrm{WM} \mathrm{T}_{1}$ estimates. In addition to errors in $\mathrm{T}_{1}$ values in general, the GM-WM contrast may be reduced, resulting in erroneous estimation of cortical thickness.

\subsection{1. $\mid B_{1}{ }^{+}$dependency of cortical $T_{1}$ estimates}

MP2RAGE settings are chosen accordingly to specific study aims: $T_{1}$ mapping vs. morphometry or a trade-off between them. For example, to remedy $\mathrm{T}_{1}$ errors, Marques et al. (2010) provided a set of sequence parameters that limits the $\mathrm{B}_{1}{ }^{+}$-dependence of the MP2RAGE sequence, while simultaneously attaining sufficient CNR per unit time for segmentation. However, residual $\mathrm{B}_{1}{ }^{+}$dependency may still persist even for optimized MP2RAGE sequence parameters, as it assumes a homogeneous $B_{1}^{+}$during $T_{1}$ quantification. Note that by comparing the current $\mathrm{B}_{1}{ }^{+}$sensitivity plot with that from the original MP2RAGE papers, the $\mathrm{B}_{1}{ }^{+}$sensitivity of the protocol chosen here is significantly increased (Marques et al., 2010; Marques and Gruetter, 2013). Part of this is likely due to the increased number of excitations per TR because of the higher resolution used in this study ( $1.0 \mathrm{~mm}$ vs. $0.7 \mathrm{~mm}$ isotropic nominal voxel size). Consecutively, using an iterative post-hoc correction method and the Sa2RAGE sequence, these residual inhomogeneities can be reduced by taking into account the spatially varying $\mathrm{B}_{1}{ }^{+}$across the brain (Eggenschwiler et al., 2012; Marques and Gruetter, 2013). While this approach resulted in enhanced visualization of thalamic nuclei and brainstem structures, the effect on the cortical $T_{1}$ values and on thickness remained unexplored.

It has been recently shown that the GM and WM $\mathrm{T}_{1}$ estimates using MP2RAGE becomes more comparable with IR data after the $\mathrm{B}_{1}{ }^{+}$correction (Kashyap et al., 2017). That is, this study provided strong evidence that $B_{1}{ }^{+}$correction is mandatory for MP2RAGE data to achieve accurate $T_{1}$ values. At 7T, using the standard NOVA Medical head coil, the transmit field inhomogeneities can be a result of the human's head eccentricity, but also due to the rapid drop off of the coil's transmit field's z-coverage (O'Brien et al., 2014). The insufficient $B_{1}^{+}$in the inferior brain regions reduces the inversion efficiency and impairs image quality, apparent by the reduced MP2RAGE intensity and subsequent significant overestimation of $\mathrm{T}_{1}$, too strong to counteract using the TR-FOCI inversion pulse and dielectric pads. On the other hand, underestimation of $\mathrm{T}_{1}$ (i.e. high $\mathrm{T}_{1} \mathrm{w}$ signal) in regions close to the posterior cingulate cortex are potentially the result of the 'central brightening' phenomenon due to constructive interference of traveling $\mathrm{B}_{1}^{+}$waves (Collins et al., 2005). Please note that the observed left-right asymmetry of 
the $\mathrm{B}_{1}{ }^{+}$field (observed in all subjects) is the result of the coil's design to improve the general homogeneity and reduce the sensitivity to head size and position within the coil (Ledden et al., 2005). This led to the observed differences in the extent of the $T_{1}$ correction between hemispheres, in particular for the parietal lobe. In the current study, we found that cortical T, was significantly over- (>30\%) or underestimated (up to 15\%), due to the strongly varying $\mathrm{B}_{1}^{+}$, in the inferior temporal and frontal lobes (i.e. low $\mathrm{B}_{1}^{+}$) or posterior cingulate cortex (i.e. high $\mathrm{B}_{1}^{+}$), respectively.

Overall, the $\mathrm{B}_{1}{ }^{+}$correction led to an improved inter-regional (but also inter-subject, data not shown) COV. That is, the variation of $\mathrm{T}_{1}$ values across the cortex is remarkably low after $\mathrm{B}_{1}{ }^{+}$correction. For a comparison of the inter-subject and scan-rescan COV with that of other (quantitative) contrasts (e.g. $\mathrm{T}_{2}{ }^{*}$ ), we refer the reader to Haast et al. (2016). The reduction of the superior-to-inferior gradient of low-to-high $\mathrm{T}_{1}$ - that led to the initially high COV across regions - resulted in a cortical $\mathrm{T}_{1}$ distribution that is more comparable with previous studies at lower field strengths and are less affected by $\mathrm{B}_{1}^{+}$ effects, revealing the typical myelin-related cortical pattern (Glasser and Van Essen, 2011; Lutti et al., 2014). However, even after taking the $B_{1}{ }^{+}$spatial profile into account, residual $T_{1}$ errors persisted across the whole cortex. In the specific case, for which the adiabatic condition was not met, such as in the most inferior part of the temporal lobe (i.e. that suffered from too low $\left.\mathrm{B}_{1}^{+}\right), \mathrm{T}_{1}$ remained artificially low. Additionally, local susceptibility gradients near these regions due to, for example, the spatial proximity to air-tissue interfaces, may hamper the inversion efficiency via $\mathrm{B}_{0}$-related problems.

\subsection{2. $\mid B_{1}{ }^{+}$and $T_{1}$ dependency of cortical thickness estimates}

Errors in the spatially varying $\mathrm{T}_{1}(\mathrm{w})$ image contrast due to $\mathrm{B}_{1}{ }^{+}$inhomogeneity hamper subsequent anatomical analyses, necessary to obtain biomarkers of cortical atrophy due to aging or disease. In this context, cortical thickness is a popular surface-based metric that is typically computed based on the minimal distance (in $\mathrm{mm}$ ) between the WM/GM and GM/CSF boundary surfaces (Fischl and Dale, 2000; Jones et al., 2000). Cortical thickness measures depend primarily on the fidelity of the underlying data to accurately place the boundary surfaces based on strong intensity gradients between tissue types. However, due to the dominant influence of myelin density on the GM-WM contrast in $T_{1}$ contrast-based images, estimation of cortical thickness based on MRI data has often been criticized, especially when its used to study brain development, aging or disease (Zilles and Amunts, 2015). For example, the myelin-related cortical variation of $\mathrm{T}_{1}$, myelinated thickness ratio, but also the metric used to compute cortical thickness, introduce an unpredictable inter-areal variation of cortical thickness values, not consistent with that obtained using histological data (Lerch and 


\section{CHAPTER 2}

Evans, 2005; Hutton et al., 2008; Rowley et al., 2015; Zilles and Amunts, 2015). As such, spatial variations in $T_{1}$ quantification accuracy and image CNR due to $B_{1}^{+}$inhomogeneities, can affect the accuracy of determining cortical thickness. To quantify this bias, a comparable analysis approach, as utilized in the study by Fujimoto et al. (2014) at 3T and 7T employing the Longitudinal analysis stream within FreeSurfer v6.0, was used. We observed a significant (i.e. $>70 \%$ ) increase in apparent cortical thickness in the inferior temporal and frontal lobes, while it decreased (i.e. up to 5\%) near the posterior cingulate cortex after taking into account the $\mathrm{B}_{1}{ }^{+}$profile. These differences originate predominantly from expansion of the pial surface, while the WM surface remained relatively stable, although on occasion it was placed slightly inwards. The observed changes in surface reconstructions imply improvement of spatial homogeneity and CNR of the GM/CSF. This is in close agreement with the work by Fujimoto et al. (2014). In it, the WM and pial surfaces based on $\mathrm{B}_{1}{ }^{+}$-uncorrected MP2RAGE data were positioned outside and inside of the reference (MEMPRAGE) surfaces, respectively, similar to our current observations. However, in contrast to the current study, Fujimoto et al. (2014) did not use the TR-FOCI pulse for the MP2RAGE acquisitions, neither at 3T nor at 7T. While they were not able to pinpoint the exact reason for these differences, a recent study showed that the use of a correct model for PVE near tissue boundaries in MP2RAGE data is crucial, and, therefore, hinted towards direct effects of $\mathrm{T}_{1}$ errors on the performance of cortical segmentations algorithms (Duché et al., 2017). The proposed PVE model takes into account the natural variation of $\mathrm{T}_{1}$ across the cortex as discussed before, leading to a better delineation of the tissue boundaries. Indeed, the present data show that the differences in surface reconstructions and cortical thickness are significantly (inversely) correlated with $\mathrm{T}_{1}$ errors, highlighting the importance of $\mathrm{B}_{1}^{+}$ correction. In other words, these results imply that cortical thickness measurements are less robust in regions where $\mathrm{B}_{1}{ }^{+}$is strongly off from its nominal value. The reduced contrast between tissue types led to pronounced differences in cortical thickness after the $\mathrm{B}_{1}^{+}$correction, within regions, but also across subjects.

In order to assess the improvement due to $\mathrm{B}_{1}{ }^{+}$correction and evaluate and benchmark our findings, we compared cortical thickness values in the current study with those of the cortical thickness model developed by Potvin et al. (2017), based on 2757 cognitively healthy controls aged 18 to 94 years. The model incorporates age, sex, estimated total intracranial volume, magnetic field strength and scanner vendor information to estimate subject-specific regional cortical thickness averages. Age, but also extrinsic factors, such as field strength and scanner platform, may affect cortical thickness estimates and are, therefore, important to take into account (Han et al., 2006; Lusebrink et al., 2013; Govindarajan et al., 2014; Potvin et al., 2017). To quantify the accuracy, we 
computed the Euclidean distance for each region between the normative data and the average cortical thickness derived from either the original or corrected data across all subjects. The $\mathrm{B}_{1}{ }^{+}$correction improved the correspondence with the normative data, especially in the problematic regions, i.e. those characterized by the largest $T_{1}$ errors. Despite these improvements, our measurements were systematically lower than assumed using the model, except for those where $B_{1}{ }^{+}$was close to the nominal value. This suggests that $B_{1}{ }^{+}$inhomogeneity is the main source for the discrepancy with the normative data. However, it is important to remember that the model does not represent the ground truth, since image biases in the model's underlying data could have led to overestimation of cortical thickness and should, therefore, be considered more as a benchmark. The thicker estimates of the cortex using the model could have potentially originated from the $\mathrm{T}_{2}{ }^{*}$ as well as PD contrast present in the data used for the model, which, in contrast, are eliminated in the current MP2RAGE data. For example, variations in cortical thickness measurements were observed after removal of the PD component from $T_{1} w$ images (Lorio et al., 2016). Nevertheless, two other important factors, both related to the spatial resolution of the input data, could have contributed to the slight discrepancy of our results to those of Potvin et al. (2017). First, in contrast to our 7T submillimeter data, the model is based on lower field strength and lower spatial resolution data. Therefore, this model may not be fully applicable to our 7T data, even though the study of Potvin et al. (2017) did not detect field strength dependency of cortical thickness estimates based on 1.5T and 3T data. Also, increased PVE, due to the lower resolution, may have slightly overestimated the cortical thickness obtained using the 1.5T and $3 \mathrm{~T}$ datasets. In accordance with this, significantly reduced thickness has previously been determined using submillimeter data compared to $1 \mathrm{~mm}^{3}$ (ME)MPRAGE data at 7T, providing evidence for this spatial resolution effect (Lusebrink et al., 2013; Zaretskaya et al., 2017). Secondly, FreeSurfer v6.0 was used in the current study, which enables analysis of the data at the native resolution $\left(0.7 \mathrm{~mm}^{3}\right)$, whereas FreeSurfer v5.3, which was used to develop the model, conforms the data (which ranged from 0.3 to $2.3 \mathrm{~mm}^{3}$ for the data used to develop the model) to $1 \mathrm{~mm}^{3}$ resolution and by this means, affecting the spatial specificity. For example, CSF could be misclassified as GM in narrow sulci and therefore lead to overestimation of the cortical thickness, such as seen for the normative data. However, similar differences were observed when analyses were repeated using FreeSurfer v5.3 for a subset of the subjects. Based on the arguments above, the observed discrepancy between the measured cortical thickness and the normative data results presumably from several factors. In case $\mathrm{B}_{1}{ }^{+}$matches its nominal value, the MP2RAGE data remains unchanged after the correction. In addition, due to the folding of the GM ribbon, partial volume effects are random and the measured cortical thickness is comparable 


\section{CHAPTER 2}

with the normative data, even if acquired with a slightly different spatial resolution. However, additional (competing) effects of $\mathrm{T}_{2}{ }^{*}$ and/or PD in model's data may lead to a higher correlation with our data in regions where $\mathrm{B}_{1}^{+}$is not close to its nominal value. Please note that this issue is not the main focus of this paper and would require a more systematic investigation.

\subsection{3. | Conclusion}

The accuracy of MRI-based measurements of cortical thickness are directly dependent on the $\mathrm{T}_{1}(\mathrm{w})$ image quality. As such, $\mathrm{B}_{1}{ }^{+}$-related inhomogeneities in UHF MRI data significantly affect cortical $T_{1}$ and thickness estimates. In the specific case of MP2RAGE data, correction for the varying $\mathrm{B}_{1}{ }^{+}$across the cortex predominantly decreased apparent $\mathrm{T}_{1}$, leading towards increased and more accurate cortical thickness measurements in the lower frontal and temporal lobe regions. Here, the automatic estimation of cortical thickness is mostly improved due to a better delineation of the GM-CSF boundary through more homogeneous apparent $\mathrm{T}_{1}$ and improved CNR. Taken together, correction for MR image imperfections harbors profound implications for clinical neuroscientific studies interested in disease- and/or age-related microstructural and morphological changes and should be taken into account when setting up imaging protocols and analysis pipelines. 


\section{5. | REFERENCES}

Ashburner, J. (2009). "Computational anatomy with the SPM software." Magn Reson Imaging 27(8): 1163-1174.

Ashburner, J. and K. J. Friston (2005). “Unified segmentation.” Neuroimage 26(3): 839-851.

Bazin, P. L., M. Weiss, J. Dinse, A. Schafer, R. Trampel and R. Turner (2014). "A computational framework for ultra-high resolution cortical segmentation at 7Tesla." Neuroimage 93 Pt 2: 201-209.

Collins, C. M., S. Li and M. B. Smith (1998). "SAR and B1 field distributions in a heterogeneous human head model within a birdcage coil. Specific energy absorption rate." Magn Reson Med 40(6): 847-856.

Collins, C. M., W. Liu, W. Schreiber, Q. X. Yang and M. B. Smith (2005). “Central brightening due to constructive interference with, without, and despite dielectric resonance." J Magn Reson Imaging 21(2): 192-196.

Dale, A. M., B. Fischl and M. I. Sereno (1999). “Cortical surface-based analysis. I. Segmentation and surface reconstruction." Neuroimage 9(2): 179-194.

De Martino, F., M. Moerel, J. Xu, P. F. van de Moortele, K. Ugurbil, R. Goebel, . . . E. Formisano (2015). "High-Resolution Mapping of Myeloarchitecture In Vivo: Localization of Auditory Areas in the Human Brain." Cereb Cortex 25(10): 3394-3405.

De Martino, F., E. Yacoub, V. Kemper, M. Moerel, K. Uludag, P. De Weerd, ... E. Formisano (2017). "The impact of ultra-high field MRI on cognitive and computational neuroimaging." Neuroimage.

Deoni, S. C., B. K. Rutt and T. M. Peters (2003). “Rapid combined T1 and T2 mapping using gradient recalled acquisition in the steady state." Magn Reson Med 49(3): 515-526.

Desikan, R. S., F. Segonne, B. Fischl, B. T. Quinn, B. C. Dickerson, D. Blacker, ... R. J. Killiany (2006). "An automated labeling system for subdividing the human cerebral cortex on MRI scans into gyral based regions of interest." Neuroimage 31(3): 968-980.

Despotovic, I., B. Goossens and W. Philips (2015). "MRI segmentation of the human brain: challenges, methods, and applications." Comput Math Methods Med 2015: 450341.

Duché, Q., H. Saint-Jalmes, O. Acosta, P. Raniga, P. Bourgeat, V. Doré, .. . O. Salvado (2017). “Partial volume model for brain MRI scan using MP2RAGE." Human Brain Mapping 38(10): 5115-5127.

Eggenschwiler, F., T. Kober, A. W. Magill, R. Gruetter and J. P. Marques (2012). “SA2RAGE: a new sequence for fast B1+ -mapping." Magn Reson Med 67(6): 1609-1619.

Fischl, B. and A. M. Dale (2000). "Measuring the thickness of the human cerebral cortex from magnetic resonance images." Proc Natl Acad Sci U S A 97(20): 11050-11055.

Fischl, B., D. H. Salat, A. J. van der Kouwe, N. Makris, F. Segonne, B. T. Quinn and A. M. Dale (2004). "Sequence-independent segmentation of magnetic resonance images." Neuroimage 23 Suppl 1: S69-84.

Fischl, B., M. I. Sereno, R. B. Tootell and A. M. Dale (1999). "High-resolution intersubject averaging and a coordinate system for the cortical surface." Hum Brain Mapp 8(4): 272-284.

Fujimoto, K., J. R. Polimeni, A. J. van der Kouwe, M. Reuter, T. Kober, T. Benner, ... L. L. Wald (2014). "Quantitative comparison of cortical surface reconstructions from MP2RAGE and multi-echo MPRAGE data at 3 and 7 T." Neuroimage 90: 60-73.

Glasser, M. F. and D. C. Van Essen (2011). “Mapping human cortical areas in vivo based on myelin content as revealed by T1- and T2-weighted MRI." J Neurosci 31 (32): 11597-11616.

Govindarajan, K. A., L. Freeman, C. Cai, M. H. Rahbar and P. A. Narayana (2014). “Effect of intrinsic and extrinsic factors on global and regional cortical thickness." PLoS One 9(5): e96429.

Gulban, O. F., M. Schneider, I. Marquardt, R. A. M. Haast and F. De Martino (2018). "A scalable method to improve gray matter segmentation at ultra high field MRI." bioRxiv. 


\section{CHAPTER 2}

Haast, R. A., D. Ivanov, E. Formisano and K. Uludag (2016). "Reproducibility and Reliability of Quantitative and Weighted T1 and T2 * Mapping for Myelin-Based Cortical Parcellation at 7 Tesla." Front Neuroanat 10: 112.

Han, X., J. Jovicich, D. Salat, A. van der Kouwe, B. Quinn, S. Czanner, ... B. Fischl (2006). “Reliability of MRI-derived measurements of human cerebral cortical thickness: the effects of field strength, scanner upgrade and manufacturer." Neuroimage 32(1): 180-194.

Helms, G., H. Dathe and P. Dechent (2008). "Quantitative FLASH MRI at 3T using a rational approximation of the Ernst equation." Magn Reson Med 59(3): 667-672.

Helms, G., K. Kallenberg and P. Dechent (2006). "Contrast-driven approach to intracranial segmentation using a combination of T2- and T1-weighted 3D MRI data sets." J Magn Reson Imaging 24(4): 790-795.

Hurley, A. C., A. Al-Radaideh, L. Bai, U. Aickelin, R. Coxon, P. Glover and P. A. Gowland (2010). "Tailored RF pulse for magnetization inversion at ultrahigh field." Magn Reson Med 63(1): 51-58.

Hutton, C., E. De Vita, J. Ashburner, R. Deichmann and R. Turner (2008). "Voxel-based cortical thickness measurements in MRI." Neuroimage 40(4): 1701-1710.

Jones, S. E., B. R. Buchbinder and I. Aharon (2000). "Three-dimensional mapping of cortical thickness using Laplace's equation." Hum Brain Mapp 11(1): 12-32.

Kashyap, S., D. Ivanov, M. Havlicek, B. A. Poser and K. Uludag (2017). "Impact of acquisition and analysis strategies on cortical depth-dependent fMRI." Neuroimage.

Lambert, C., A. Lutti, G. Helms, R. Frackowiak and J. Ashburner (2013)."Multiparametric brainstem segmentation using a modified multivariate mixture of Gaussians." Neuroimage Clin 2: 684-694.

Ledden, P., P. Gelderen and J. Duyn (2005). Birdcage volume transmit, eight channel receive array system for brain imaging at 7T. Proceedings of the 13th Annual Meeting of ISMRM, Miami Beach, FL.

Lerch, J. P. and A. C. Evans (2005). “Cortical thickness analysis examined through power analysis and a population simulation." Neuroimage 24(1): 163-173.

Liu, J. V., N. A. Bock and A. C. Silva (2011). “Rapid high-resolution three-dimensional mapping of T1 and age-dependent variations in the non-human primate brain using magnetization-prepared rapid gradient-echo (MPRAGE) sequence." Neuroimage 56(3): 1154-1163.

Lorio, S., F. Kherif, A. Ruef, L. Melie-Garcia, R. Frackowiak, J. Ashburner, . . B. Draganski (2016). "Neurobiological origin of spurious brain morphological changes: A quantitative MRI study." Hum Brain Mapp 37(5): 1801-1815.

Lusebrink, F., A. Wollrab and O. Speck (2013). "Cortical thickness determination of the human brain using high resolution 3T and 7T MRI data." Neuroimage 70: 122-131.

Lutti, A., F. Dick, M. I. Sereno and N. Weiskopf (2014). “Using high-resolution quantitative mapping of R1 as an index of cortical myelination." Neuroimage 93 Pt 2: 176-188.

Lutti, A., C. Hutton, J. Finsterbusch, G. Helms and N. Weiskopf (2010). "Optimization and validation of methods for mapping of the radiofrequency transmit field at 3T." Magn Reson Med 64(1): 229-238.

Marques, J. P. and R. Gruetter (2013). "New developments and applications of the MP2RAGE sequence--focusing the contrast and high spatial resolution R1 mapping." PLoS One 8(7): e69294.

Marques, J. P., D. Khabipova and R. Gruetter (2017). "Studying cyto and myeloarchitecture of the human cortex at ultra-high field with quantitative imaging: R1, R2 $\left(^{*}\right)$ and magnetic susceptibility." Neuroimage 147: 152-163.

Marques, J. P., T. Kober, G. Krueger, W. van der Zwaag, P. F. Van de Moortele and R. Gruetter (2010). "MP2RAGE, a self bias-field corrected sequence for improved segmentation and T1-mapping at high field." Neuroimage 49(2): 1271-1281.

Marques, J. P. and D. G. Norris (2017). "How to choose the right MR sequence for your research question at 7T and above?" Neuroimage. 
Mugler, J. P., 3rd and J. R. Brookeman (1990). “Three-dimensional magnetization-prepared rapid gradient-echo imaging (3D MP RAGE)." Magn Reson Med 15(1): 152-157.

O'Brien, K. R., A. W. Magill, J. Delacoste, J. P. Marques, T. Kober, H. P. Fautz, ... G. Krueger (2014). “Dielectric pads and low- B1+ adiabatic pulses: complementary techniques to optimize structural T1 w whole-brain MP2RAGE scans at 7 tesla." J Magn Reson Imaging 40(4): 804-812.

O'Brien, K., G. Krueger, F. Lazeyras, R. Gruetter and A. Roche (2013). A simple method to denoise MP2RAGE. Proceedings of the 21th scientific meeting, International Society for Magnetic Resonance in Medicine, Salt Lake City.

Padormo, F., A. Beqiri, J. V. Hajnal and S. J. Malik (2016). “Parallel transmission for ultrahigh-field imaging." NMR Biomed 29(9): 1145-1161.

Pohmann, R. and K. Scheffler (2013). "A theoretical and experimental comparison of different techniques for B(1) mapping at very high fields." NMR Biomed 26(3): 265-275.

Pohmann, R., O. Speck and K. Scheffler (2016). "Signal-to-noise ratio and MR tissue parameters in human brain imaging at 3, 7, and 9.4 tesla using current receive coil arrays." Magn Reson Med 75(2): 801-809.

Polimeni, J. R., V. Renvall, N. Zaretskaya and B. Fischl (2017). "Analysis strategies for high-resolution UHF-fMRI data." Neuroimage.

Potvin, O., L. Dieumegarde, S. Duchesne and I. Alzheimer's Disease Neuroimaging (2017). "Normative morphometric data for cerebral cortical areas over the lifetime of the adult human brain." Neuroimage 156: 315-339.

Reuter, M. and B. Fischl (2011). "Avoiding asymmetry-induced bias in longitudinal image processing." Neuroimage 57(1): 19-21.

Reuter, M., N. J. Schmansky, H. D. Rosas and B. Fischl (2012). “Within-subject template estimation for unbiased longitudinal image analysis." Neuroimage 61(4): 1402-1418.

Rowley, C. D., P. L. Bazin, C. L. Tardif, M. Sehmbi, E. Hashim, N. Zaharieva, ... N. A. Bock (2015). "Assessing intracortical myelin in the living human brain using myelinated cortical thickness." Front Neurosci 9: 396.

Smith, S. M., M. Jenkinson, M. W. Woolrich, C. F. Beckmann, T. E. Behrens, H. Johansen-Berg, ... P. M. Matthews (2004). "Advances in functional and structural MR image analysis and implementation as FSL." Neuroimage 23 Suppl 1: S208-219.

Stuber, C., M. Morawski, A. Schafer, C. Labadie, M. Wahnert, C. Leuze, .. R. Turner (2014). "Myelin and iron concentration in the human brain: a quantitative study of MRI contrast." Neuroimage 93 Pt 1: 95-106.

Teeuwisse, W. M., W. M. Brink and A. G. Webb (2012). "Quantitative assessment of the effects of high-permittivity pads in 7 Tesla MRI of the brain." Magn Reson Med 67(5): 1285-1293.

Tustison, N. J., B. B. Avants, P. A. Cook, Y. Zheng, A. Egan, P. A. Yushkevich and J. C. Gee (2010). “N4ITK: improved N3 bias correction." IEEE Trans Med Imaging 29(6): 1310-1320.

Ugurbil, K. (2017). "Imaging at ultrahigh magnetic fields: History, challenges, and solutions." Neuroimage.

Van de Moortele, P. F., C. Akgun, G. Adriany, S. Moeller, J. Ritter, C. M. Collins, ... K. Ugurbil (2005). "B(1) destructive interferences and spatial phase patterns at $7 \mathrm{~T}$ with a head transceiver array coil." Magn Reson Med 54(6): 1503-1518.

Van de Moortele, P. F., E. J. Auerbach, C. Olman, E. Yacoub, K. Ugurbil and S. Moeller (2009). "T1 weighted brain images at 7 Tesla unbiased for Proton Density, T2* contrast and RF coil receive B1 sensitivity with simultaneous vessel visualization." Neuroimage 46(2): 432-446.

Vaughan, J. T., M. Garwood, C. M. Collins, W. Liu, L. DelaBarre, G. Adriany, ... K. Ugurbil (2001). "7T vs. 4T: RF power, homogeneity, and signal-to-noise comparison in head images." Magn Reson Med 46(1): 24-30.

Viviani, R., E. D. Pracht, D. Brenner, P. Beschoner, J. C. Stingl and T. Stocker (2017). “Multimodal 


\section{CHAPTER 2}

MEMPRAGE, FLAIR, and [Formula: see text] Segmentation to Resolve Dura and Vessels from Cortical Gray Matter." Front Neurosci 11: 258.

Weiskopf, N., A. Lutti, G. Helms, M. Novak, J. Ashburner and C. Hutton (2011). “Unified segmentation based correction of R1 brain maps for RF transmit field inhomogeneities (UNICORT)." Neuroimage 54(3): 2116-2124.

Zaretskaya, N., B. Fischl, M. Reuter, V. Renvall and J. R. Polimeni (2017). “Advantages of cortical surface reconstruction using submillimeter 7 T MEMPRAGE." Neurolmage.

Zilles, K. and K. Amunts (2015). Anatomical Basis for Functional Specialization. fMRI: From Nuclear Spins to Brain Functions. K. Uludag, K. Ugurbil and L. Berliner. Boston, MA, Springer US: 27-66. 


\section{6. | SUPPLEMENTARY FIGURES}
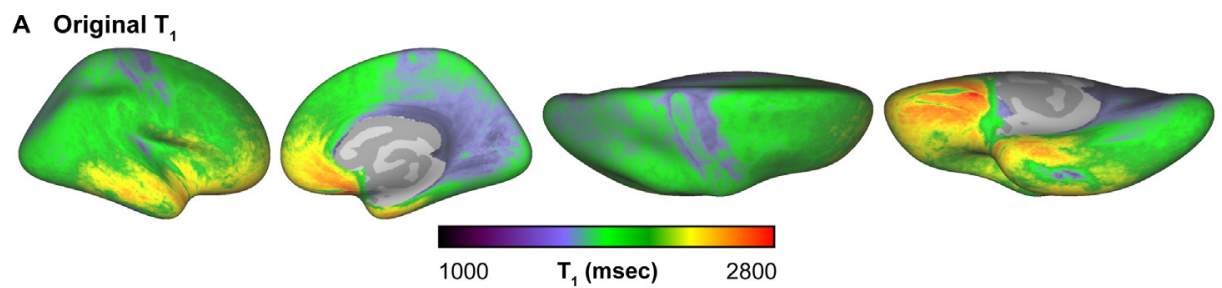

B Corrected $\mathbf{T}_{1}$
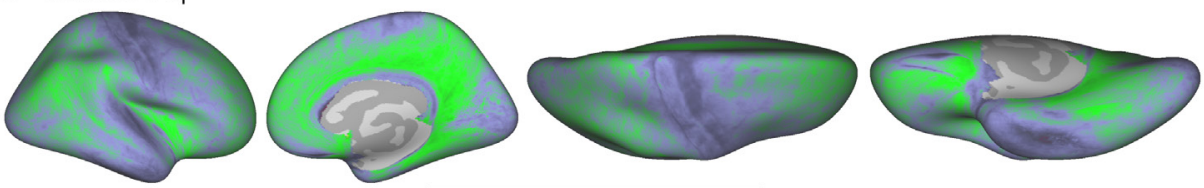

C $T_{1}$ difference
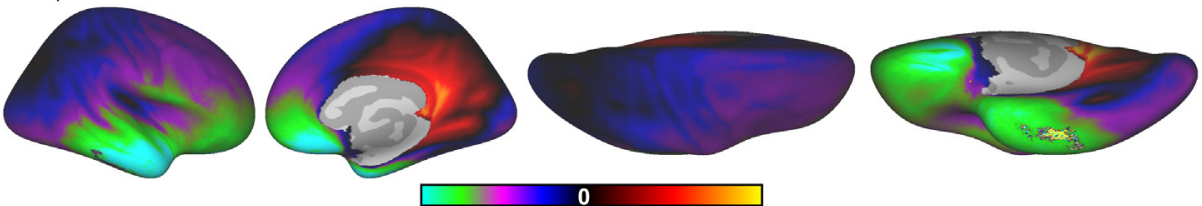

D Statistical difference
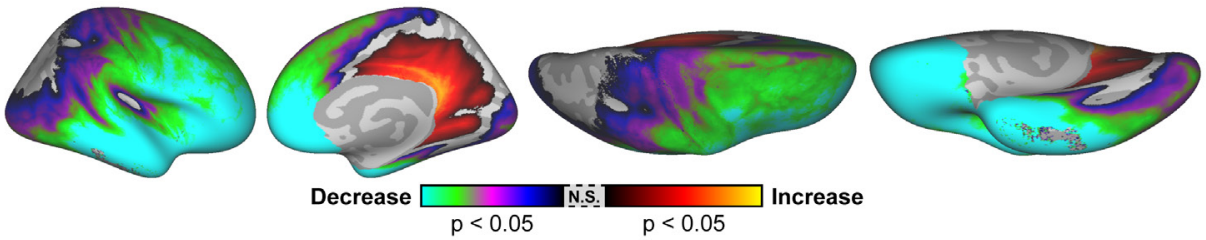

E $\mathbf{B}_{1}^{+}$
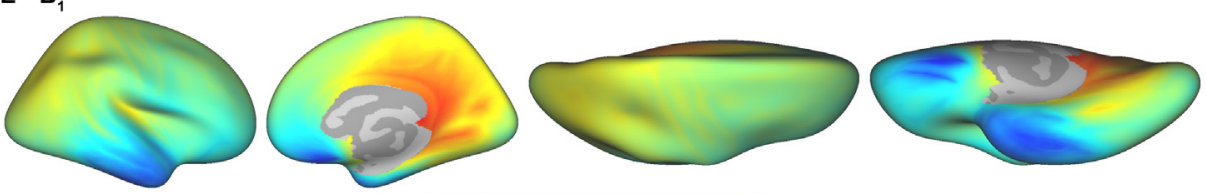

0

$\mathrm{B}_{1}^{+}(\times$nominal $\alpha) \quad 1.6$

Fig. S2.1. | Average cortical $T$, surface maps for right hemisphere. Original (A) and corrected (B) $T$, (msec), $T_{1}$ difference $(\%, C)$, statistical surface map (D) and $B_{1}^{+}$volume ( $\times$nominal value, E) were mapped onto an inflated right hemisphere surface and averaged across all subjects. Only vertices with a significant (FDR-corrected $p<0.05$ ) decrease (blue/green) or increase (red/yellow) of $T_{1}$ are highlighted in D and gray vertices are non-significant (N.S.). 


\section{CHAPTER 2}
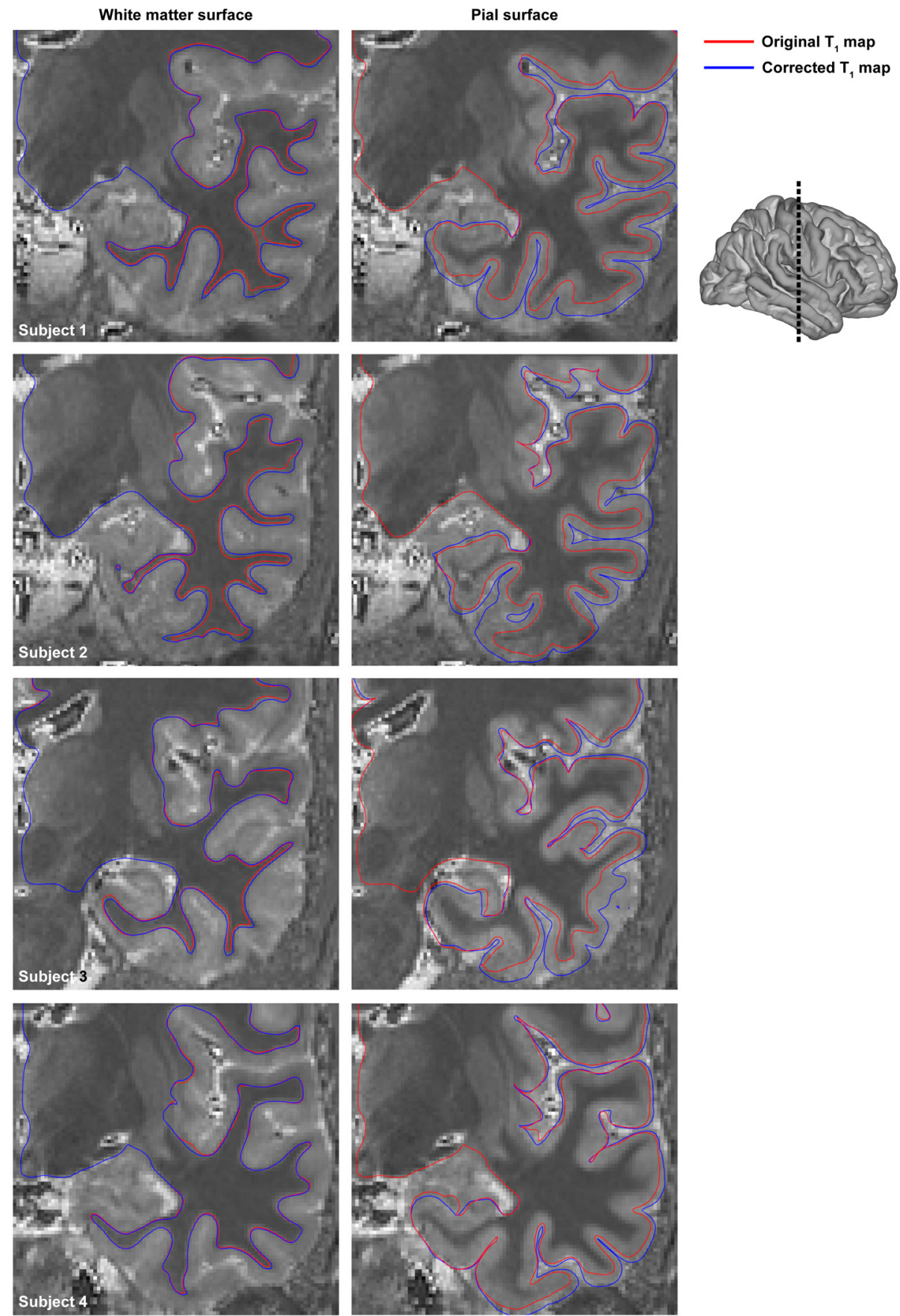

Fig. S2.2. White matter and pial surface reconstructions. Example white matter (left) and pial (right) surface reconstructions based on the original (red lines) and corrected (blue) $T_{1}$ maps are shown for several subjects (rows). 


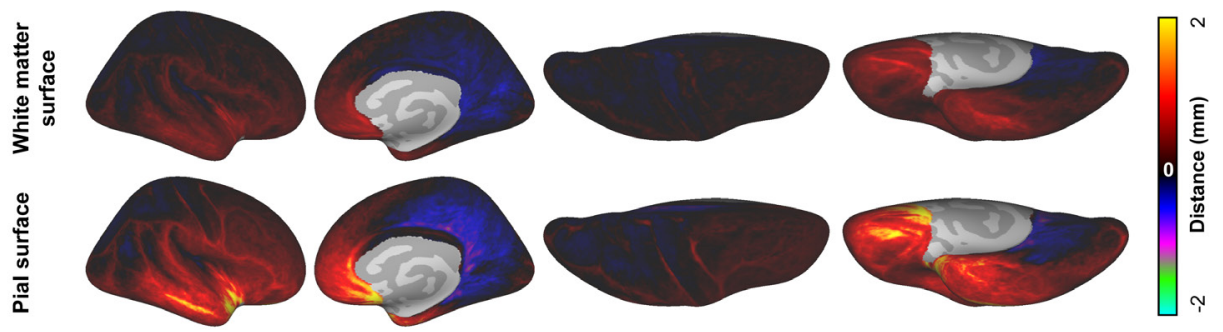

Fig. S2.3. | Surface reconstruction difference for right hemisphere. The spatial difference $(\mathrm{mm})$ between both surfaces (white matter: top and pial: bottom), based on the original and corrected data, are mapped onto an inflated right hemisphere surface.

\section{A Original cortical thickness}
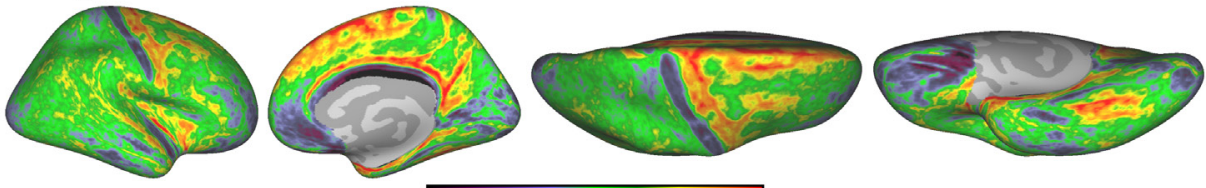

1 Cortical thickness (mm) 3

B Corrected cortical thickness
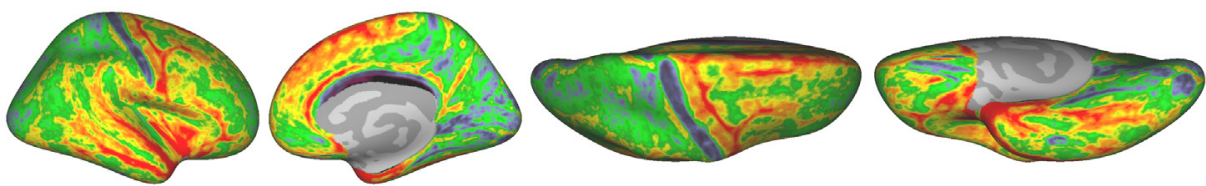

C Cortical thickness difference
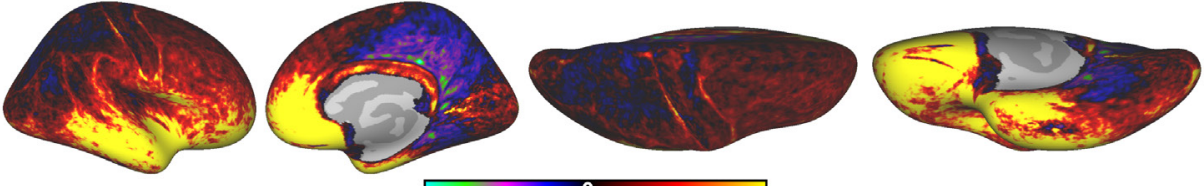

D Statistical difference
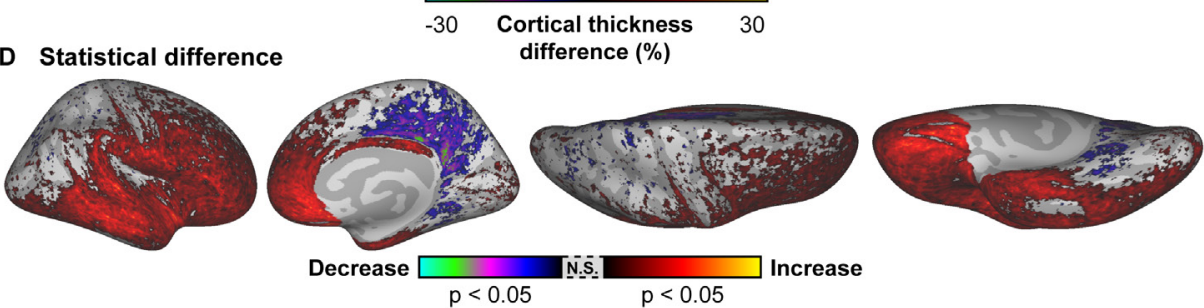

Fig. S2.4. | Average cortical thickness surface maps for right hemisphere. Original (A) and corrected (B) cortical thickness $(\mathrm{mm})$, cortical thickness difference $(\%, C)$ and statistical difference $(D)$ were mapped onto an inflated right hemisphere surface and averaged across all subjects. Only vertices with a significant (FDR-corrected $p<0.05$ ) decrease (blue/green) or increase (red/yellow) of cortical thickness are highlighted in D and gray vertices are non-significant (N.S.). 


\section{CHAPTER 2}

- Cortical region ----- Linear fit $( \pm \mathbf{9 5 \%}$ Cls)

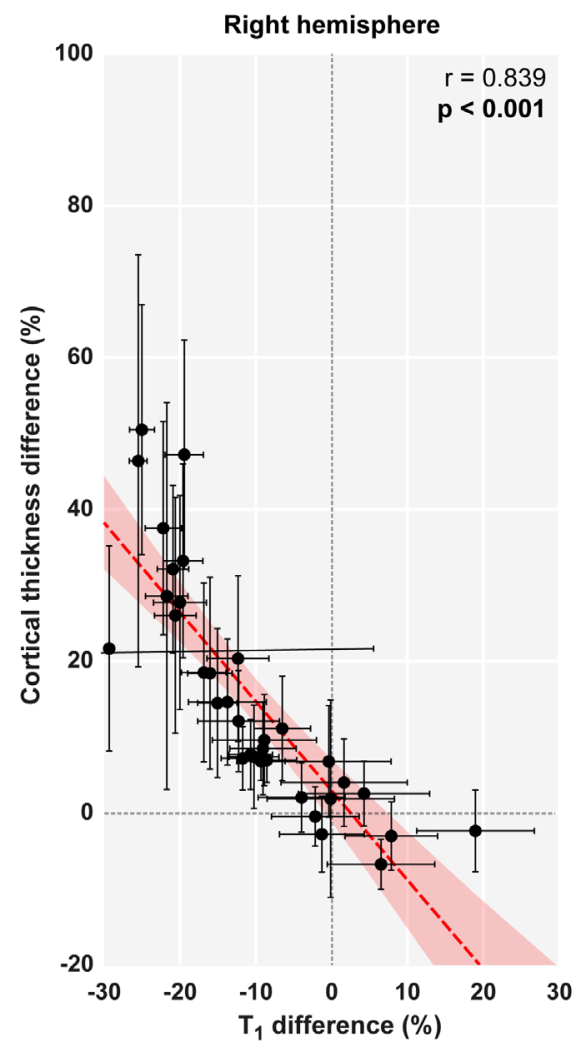

Fig. S2.5. | Regional $T_{1}$ and cortical thickness changes. Across subjects average $T_{1}(x$-axis) and cortical thickness (y-axis) changes (\% \pm S.D.) are plotted across all regions (black dots) for the right hemisphere. Dashed red lines represent the best fit $\pm 95 \% \mathrm{Cl}$. Boldface $p$-values indicate a significant correlation. 
A

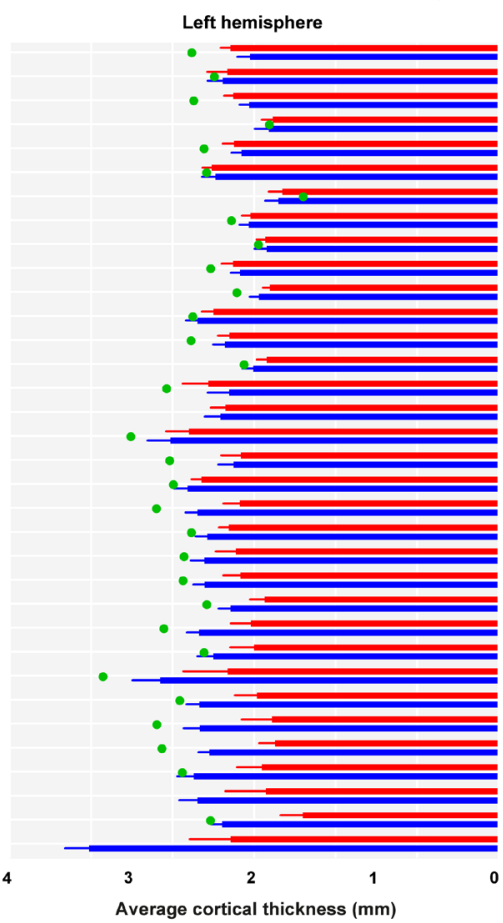

B

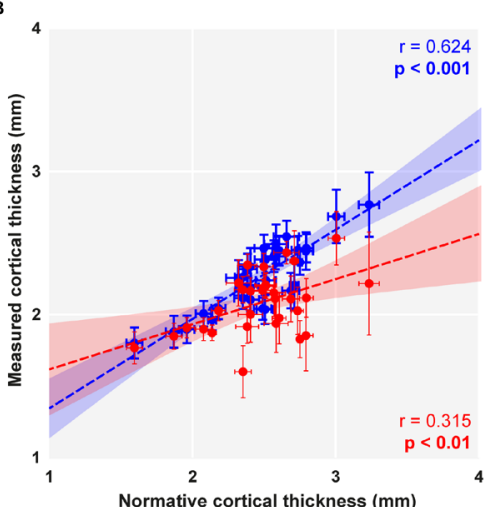

— Corrected $\mathrm{T}_{1}$ map - Normative data
isthmuscingulate
ransversetemporal
posteriorcingulate cuneus
inferiorparietal
paracentral
pericalcarine
superiorparietal
lingual
precuneus
lateraloccipital
precentral
supramarginal
postcentral
parahippocampal
bankssts
insula
caudalanteriorcingulate
superiorfrontal
superiortemporal
caudalmiddlefrontal
parsopercularis
fusiform
rostralmiddlefrontal
middletemporal
parstriangularis
entorhinal
lateralorbitofrontal
rostralanteriorcingulate
inferiortemporal
parsorbitalis
frontalpole
medialorbitofrontal
temporalpole
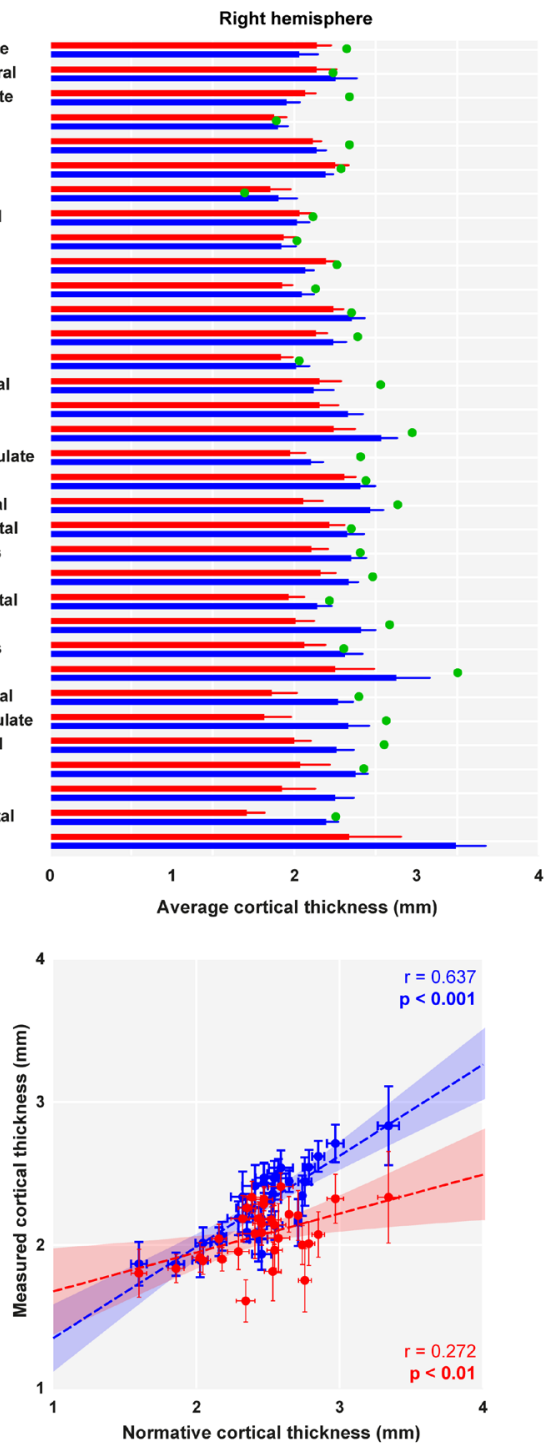

- Cortical region ----- Linear fit $( \pm 95 \% \mathrm{Cls})$

Original $\mathrm{T}_{1}$ map

- Corrected $T_{1}$ map

Fig. S2.6. | Comparison between regional cortical thickness averages with normative data. Original (red lines) and corrected (blue) subject-wise ( \pm S.D.) cortical thickness averages $(x$-axis, $m m$ ) are plotted across all regions ( $y$-axis) for both the left (left column) and right (right) hemispheres ( $A$ ). Green dots indicate the regional averages obtained using the normative data. Regions are sorted based on the original $T$, map (see Fig. 2.3). In B, normative (x-axis) and measured original (red dots) and corrected (blue dots, $y$-axis) cortical thickness values are plotted region-wise ( $\pm S$ S.D.). Dashed lines represent the best fit $\pm 95 \% \mathrm{Cl}$. Boldface $p$-values indicate a significant correlation. 


\section{REPRODUCIBILITY AND RELIABILITY OF QUANTITATIVE AND WEIGHTED T, AND $\mathrm{T}_{2}{ }^{*}$ MAPPING FOR MYELIN-BASED CORTICAL PARCELLATION AT 7 TESLA}

ROY A.M. HAAST

DIMO IVANOV

ELIA FORMISANO

\& KÂMIL ULUDAĞ

\section{Corresponding publication:}

"Reproducibility and Reliability of Quantitative and Weighted $T_{1}$ and $T_{2}{ }^{*}$ Mapping for Myelin-Based Cortical Parcellation at 7 Tesla". Front Neuroanat. Nov 18;10:112. 


\section{CHAPTER 3}

\section{ABSTRACT}

Different magnetic resonance (MR) parameters, such as $R_{1}\left(=1 / T_{1}\right)$ or $T_{2}^{*}$, have been used to visualize non-invasively the myelin distribution across the cortical sheet. Myelin contrast is consistently enhanced in the primary sensory and some higher order cortical areas (such as MT or the cingulate cortex), which renders it suitable for subject-specific anatomical cortical parcellation. However, no systematic comparison has been performed between the previously proposed MR parameters, i.e. the longitudinal and transversal relaxation values (or their ratios), for myelin mapping at 7 Tesla. In addition, usually these MR parameters are acquired in a non-quantitative manner ("weighted" parameters). Here, we evaluated the differences in 'parcellability', contrast-to-noise ratio (CNR) and inter- and intra-subject variability and reproducibility, respectively, between high-resolution cortical surface maps based on these weighted MR parameters and their quantitative counterparts in ten healthy subjects. All parameters were obtained in a similar acquisition time and possible transmit- or receive-biases were removed during post-processing. It was found that CNR per unit time and parcellability were lower for the transversal compared to the longitudinal relaxation parameters. Further, quantitative $\mathrm{R}_{1}$ was characterized by the lowest interand intra-subject coefficient of variation ( $5.53 \%$ and $1.63 \%$, respectively), making $R_{1}$ a better parameter to map the myelin distribution compared to the other parameters. Moreover, quantitative MRI approaches offer the advantage of absolute rather than relative characterization of the underlying biochemical composition of the tissue, allowing more reliable comparison within subjects and between healthy subjects and patients. Finally, we explored two parcellation methods (thresholding the MR parameter values vs. surface gradients of these values) to determine areal borders based on the cortical surface pattern. It is shown that both methods are partially observer-dependent, needing manual interaction (i.e. choice of threshold or connecting high gradient values) to provide unambiguous borders.

\section{Acknowledgments}

This work was supported by Maastricht University, the Netherlands Organization for Scientific Research (NWO; VIDI grant 452-11-002 to K.U.) and Technology Foundation STW (12724 to E.F.). The authors are indebted to Prof. Dr. Andrew Webb (Leiden University Medical Centre, Leiden, Netherlands) and Dr. José Marques (Donders Institute for Brain, Cognition and Behaviour, Nijmegen, Netherlands) for providing the dielectric pads and the MATLAB code to perform the post-hoc T1 correction used in this study, respectively. 


\section{1. | INTRODUCTION}

The brain can be partitioned into distinct functional and anatomical areas based on functional specificity and histological markers, such as cyto-architecture, receptor-architecture and cortical myelin distribution (Brodmann, 1909; Zilles and Amunts, 2009; Nieuwenhuys et al., 2014). In particular, the distribution of cortical myelin (i.e. myeloarchitecture) is highly suitable for parcellating the brain (Brodmann, 1909; Vogt and Vogt, 1919; Nieuwenhuys et al., 2014). A recent paper by Glasser \& Van Essen (2011) suggested utilizing MR imaging to partition the cortex based on myeloarchitecture (Geyer et al., 2011; Glasser and Van Essen, 2011; Glasser et al., 2016). It has been proposed that myelin and other compounds colocalized to myelin (such as lipids (e.g. cholesterol), free and myelin-bound water and iron) influence the longitudinal $\left(T_{1}\right)$ and transverse $\left(\mathrm{T}_{2}\right.$ and $\left.\mathrm{T}_{2}{ }^{*}\right)$ relaxation times (Koenig, 1991; Miot-Noirault et al., 1997; Schmierer et al., 2004; Callaghan et al., 2015).

The relative contribution of the individual compounds to the different MR parameters remains an active and important area of research (e.g. Rooney et al. (2007); Stuber et al. (2014); Callaghan et al. (2015)). Water content (i.e. proton density), myelin (or more generally macromolecules) and iron have been identified as major determinants of $T_{1}$ and $\mathrm{T}_{2}{ }^{*}$ contrast across the brain and their distributions overlap significantly in many regions, especially in the cortex. The relative contributions of iron and myelin to the $\mathrm{T}_{1}$ and $\mathrm{T}_{2}{ }^{*}$ parameters, however, differs; with myelin being the dominant contrast source in $\mathrm{T}_{1}$ (Rooney et al., 2007; Stuber et al., 2014; Callaghan et al., 2015) and iron in $\mathrm{T}_{2}{ }^{*}$ maps (Fukunaga et al., 2010; Langkammer et al., 2010; Stuber et al., 2014). Stuber et al. (2014) report that iron has an average contribution of $10 \%$ to $T_{1}$ (acquired at a field strength of 7 Tesla [7T]) in white matter and 36\% in grey matter, while Callaghan et al. (2015) show that myelination is a better predictor of $T_{1}$ variation than iron content. In contrast, the iron density strongly determines the $\mathrm{T}_{2}{ }^{*}$ contrast not only in grey, but also in white matter in addition to the myelin concentration (Fukunaga et al., 2010; Stuber et al., 2014). Furthermore, the orientation of the myelinated fibers with respect to the magnetic field also influences the $\mathrm{T}_{2}{ }^{*}$ contrast (Cohen-Adad et al., 2012).

Several studies showed that differences in MR intensity levels correlated well with observations in histological myelin-stained sections (Fatterpekar et al., 2002; Eickhoff et al., 2005; Bock et al., 2009; Geyer et al., 2011; Glasser et al., 2014; Stuber et al., 2014). By combining MRI with histology, these studies showed high concentrations of intra-cortical myelin in the primary areas, including the motor (M1), somatosensory (S1), visual 


\section{CHAPTER 3}

(V1) and auditory (AC) cortices, but also in some higher order areas, such as $\mathrm{MT}^{1}$.

Several in vivo MRI mapping approaches were tested so far to study myeloarchitecture in humans, covering different magnetic fields (1.5T to $7 \mathrm{~T}$ ) and MR parameters ( $T_{1}$-weighted $\left[T_{1} w\right]$, quantitative $T_{1}, T_{2}{ }^{(*)}$-weighted ${ }^{2}\left[T_{2}{ }^{(*)} w\right]$, quantitative $T_{2}{ }^{(*)}$ and $T_{1} w /$ $\left.\mathrm{T}_{2}{ }^{(*)} \mathrm{W}\right)$. Cortical areas with higher myelination showed increased intensity levels in $T_{1} w$ (Bock et al., 2013) and quantitative $R_{1}\left(=1 / T_{1}\right.$ ) images (Sigalovsky et al., 2006; Geyer et al., 2011; Dick et al., 2012; Sereno et al., 2013; Lutti et al., 2014) and reduced intensity in quantitative $T_{1}$ (Tardif et al., 2015) and $T_{2}{ }^{*}$ images (Cohen-Adad et al., 2012). This implies that by computing the ratio of $T_{1} w$ and $T_{2}{ }^{(*)} W$ image (i.e. $T_{1} w / T_{2}{ }^{(*)} w$ ratio), the contrast between the heavily myelinated areas and other regions can be enhanced (Glasser and Van Essen, 2011; De Martino et al., 2014). At the same time, this calculation eliminates the presence of receive bias fields.

When interpreting these myelin-related maps, it is important to recognize that $\mathrm{T}_{1} \mathrm{w}$ or $\mathrm{T}_{2}{ }^{(*)} \mathrm{W}$ images are not quantitative markers of the underlying biochemical composition of the tissue, but are also influenced by the MRI sequence parameters, see for example Bock et al. (2013) and Lorio et al. (2016). MRI sequences utilized for weighted imaging might even mix different basic MRI parameters (Glasser et al., 2014). Moreover, the hardware setup, including the radiofrequency (RF) transmit and receive coils, influences not only the signal-to-noise ratio (SNR), but also the local image contrast. As a result, weighted images might incorporate contributions from transmit and receive RF fields in addition to proton density, relaxation rates, macromolecule concentrations and the MR parameter of interest. This may also hold for some quantitative approaches, which do not fully account for transmit inhomogeneities (e.g. see Marques et al. 2010). These issues are of particular importance when performing large-scale multi-site or longitudinal clinical studies that focus on, for example, quantitative differences in cortical myelin distribution reflecting microstructural changes due to experience, aging or disease (Draganski et al., 2011; Focke et al., 2011; Freund et al., 2013; Grydeland et al., 2013; Weiskopf et al., 2013; Callaghan et al., 2014; Droby et al., 2015).

These limitations can be tackled through a quantitative mapping approach by using, for example, the magnetization prepared 2 rapid acquisition gradient echoes (MP2RAGE) sequence (Marques et al., 2010). In addition, a multi-echo (ME) gradi-

$1 \quad$ Note that although myelin is not the only contributor to the MRI parameters investigated, following the current terminology, we associate the cortical distribution of $T_{1}$ and $T_{2}^{*}$ values with myelin patterns.

2 In the following, $T_{2}^{(*)}$ represents both $T_{2}$ and $T_{2}^{*}$ and the weighted approaches are denoted by the index " $w$ ". 
ent-recalled echo imaging (GRE) sequence allows to obtain quantitative $\mathrm{T}_{2}{ }^{*}$-maps by applying a mono-exponential fit (Cohen-Adad et al., 2012). The quantitative MRI maps are, however, also not necessarily free from effects unrelated to the underlying microstructural properties of the brain. For example, $\mathrm{T}_{2}{ }^{*}$ maps are sensitive to non-local static field inhomogeneities near air-tissue interfaces, whereas $T_{1}$ maps obtained with the MP2RAGE sequence (Marques et al., 2010) might be influenced by transmit field $\left(\mathrm{B}_{1}{ }^{+}\right)$inhomogeneities (Eggenschwiler et al., 2012). It is important to note that their weighted imaging counterparts have comparable field-inhomogeneity sensitivities, in addition to the ones mentioned earlier.

The different quantitative and non-quantitative MR parameters used so far for in vivo myelin mapping have not been systemically compared. The primary goal of the present study is, thus, to investigate the quantitative differences between several previously proposed MRI parameters $\left(T_{1} w, R_{1}, T_{2}{ }^{*} w_{1} T_{2}{ }^{*}, T_{1} w / T_{2}{ }^{*} w, R_{1} / T_{2}{ }^{*}\right)$ using $7 T$ in terms of their variation across a group of healthy subjects and their reproducibility when a subject is scanned again. These are referred to as inter-subject coefficient of variation $(\mathrm{COV})$ and intra-subject $\mathrm{COV}$, respectively. The latter should ideally be low (i.e. low intra-subject myelination scan-rescan variability), while the former examines the effect of differential (inter-subject) myelination levels on each parameter and corresponding parcellability. Marques et al. (2010) showed high reproducibility of absolute $\mathrm{T}_{1}$ values acquired using the MP2RAGE sequence across multiple subjects and within one subject using different scanning parameters and scanners (e.g. 3T vs. 7T). Intra-subject COVs of $\mathrm{T}_{1}$ values obtained using MP2RAGE in deep-gray matter (dGM) regions ranged from $2.08 \%$ in the pulvinar to $2.89 \%$ in the substantia nigra (Okubo et al., 2016). The inter-subject reproducibility of cortical $\mathrm{T}_{2}{ }^{*}$ maps acquired using a ME-GRE sequence was around $1.66 \%$ (Govindarajan et al., 2015), while a higher inter-site (applying the same sequence on the same subject at two different scanning sites) COV was observed for $R_{2}{ }^{*}\left(1 / T_{2}^{*} ; 20.3 \%\right)$ and $T_{1} w(15.2 \%)$ maps. Lower inter-site COV was found for $R_{1}(6 \%)$ maps. Note, that these inter-site COVs were based on lower-resolution (i.e. $1 \mathrm{~mm}^{3}$ voxel-size) $R_{1}, T_{1} w$ and $R_{2}{ }^{*}$ maps acquired simultaneously (i.e. multi-parameter mapping, see Weiskopf et al. (2013)) using 3T and therefore differ from the maps acquired using the MP2RAGE and/or ME-GRE sequences.

Compared to 3T, 7T allows the acquisition of sub-millimeter resolution data within a shorter time frame and without significant penalties to the signal-to-noise ratio (SNR) and contrast-to-noise ratio (CNR). In the currently applied scanning protocol, we aimed at as short as possible total scanning time while maintaining reasonable data quality at sub-millimeter resolution $\left(0.7 \mathrm{~mm}^{3}\right)$ and evaluated SNR and CNR values and their 


\section{CHAPTER 3}

spatial distribution for each parameter. Note that the $\mathrm{T}_{1} \mathrm{w} / \mathrm{T}_{2} \mathrm{w}$ approach by Glasser and Van Essen (2011) is not included in the present investigation as acquiring whole-brain $\mathrm{T}_{2}$-weighted images at $7 \mathrm{~T}$ is challenging due to inhomogeneous transmit profiles, power deposition limitations and long acquisition times. In addition, $\mathrm{T}_{2}{ }^{*}$-weighted imaging has proven to be a comparable alternative for whole-brain high-resolution imaging of cortical myelination at 7T (Cohen-Adad et al., 2012; De Martino et al., 2014). However, $\mathrm{T}_{2}$ imaging at $7 \mathrm{~T}$ may become more feasible as parallel transmission becomes routinely used.

In addition, the current study addresses several issues related to myelin-driven parcellation of the cortex using MR parameters. First, we quantify how reliably a specific vertex value can be parcellated based on its variation within subjects and the shape of the global distribution. Second, we compare - in the same subjects - the overall covariance between (weighted and quantitative) $R_{1}$ (or $T_{1} w$ ) and $T_{2}{ }^{*}$ and between both ratio images, along the cortical surface and at different cortical depths. Although we expect similar myelin-related patterns across all parameters as in previous studies, we hypothesize that, nevertheless, differences might exist since each MR parameter is potentially affected differently by the various microstructural properties, specific biases and noise. Second, as cortical parcellation requires the definition of borders between regions, we compare two widely used methods, based on threshold contours and surface gradients, to explore whether objective criteria for cortical parcellation can be derived or whether ambiguity is present and to what extent manual interaction is necessary.

\section{2. | MATERIALS \& METHODS}

\subsection{1. | Subjects and data acquisition}

Ten healthy volunteers (age $=29.7 \pm 6.3$, between 24 and 42 years old, 7 females) were included in this study. All subjects gave written informed consent in accordance with the Declaration of Helsinki. The protocol was approved by the Ethical Committee of the Faculty of Psychology and Neuroscience, University of Maastricht, the Netherlands. MR data were acquired using a whole-body 7T magnet (Siemens Medical Systems, Erlangen, Germany) and a 32-channel phased-array head coil (Nova Medical, Wilmington, USA). High resolution ( $0.7 \mathrm{~mm}$ isotropic nominal voxel size) whole-brain quantitative $T_{1}\left(T_{1}\right)$ and $T_{1}$-weighted $\left(T_{1} w\right)$ images were obtained with the 3D MP2RAGE sequence (Marques et al., 2010) with the following parameters: TR/TE $=5000 / 2.47 \mathrm{~ms}$, $\mathrm{TI}_{1} / \mathrm{TI}_{2}=900 / 2750 \mathrm{~ms}, \mathrm{a}_{1} / \mathrm{a}_{2}=5^{\circ} / 3^{\circ}$ and generalized autocalibrating partially parallel acquisitions (GRAPPA) factor $=3$ in the phase-encoding (PE) direction (anterior-pos- 
terior) with 24 references lines. Other acquisition parameters were: 240 sagittal slices, field of view $(F O V)=224 \times 224 \mathrm{~mm}$, matrix $=320 \times 320 \times 240,6 / 8$ partial Fourier in PE direction, non-selective RF excitation, readout bandwidth (BW) $=250 \mathrm{~Hz} /$ pixel, readout sample spacing $=6.9 \mathrm{~ms}$ and total acquisition time of 8:02 $\mathrm{min}$. Whole-brain $\mathrm{B}_{1}^{+}$maps ( $2 \mathrm{~mm}$ isotropic nominal voxel size) were obtained using the saturation-prepared with 2 rapid gradient echoes (SA2RAGE) sequence (Eggenschwiler et al., 2012) with the following parameters: TR/TE $=2400 / 0.78 \mathrm{~ms}, \mathrm{TD}_{1} / \mathrm{TD}_{2}=580 / 1800 \mathrm{~ms}, \mathrm{a}_{1} / \mathrm{a}_{2}$ $=4^{\circ} / 11^{\circ}$ and GRAPPA factor $=2$ in PE direction (anterior-posterior) with 24 references lines. Other acquisition parameters were: 88 sagittal slices, FOV $=256 \times 256 \mathrm{~mm}$, matrix $=128 \times 128 \times 96,6 / 8$ partial Fourier in PE direction, non-selective RF excitation, $\mathrm{BW}=1300 \mathrm{~Hz} /$ pixel, readout sample spacing $=2.2 \mathrm{~ms}$ and total acquisition time of 2:16 min. Quantitative $T_{2}{ }^{*}\left(T_{2}{ }^{*}\right)$ and $T_{2}{ }^{*}$-weighted $\left(T_{2}{ }^{*} \mathrm{w}\right)$ images $(0.7 \mathrm{~mm}$ isotropic nominal voxel size) were obtained from a multi-echo 3D GRE sequence with the following parameters: $\mathrm{TR}=33 \mathrm{~ms}, \mathrm{TE}_{1} / \mathrm{TE}_{2} / \mathrm{TE}_{3} / \mathrm{TE}_{4}=2.53 / 7.03 / 12.55 / 20.35 \mathrm{~ms}, \mathrm{a}_{1}=11^{\circ}$ and GRAPPA factor $=2$ in PE direction (left-right) with 30 references lines. Other acquisition parameters were: 208 axial slices, FOV $=224 \times 159$, matrix $=320 \times 227 \times 208$, $6 / 8$ partial Fourier in phase and slice direction, slab-selective RF excitation, readout BWs $=290 / 210 / 170 / 90 \mathrm{~Hz} /$ pixel (different BWs were used to match SNR between the individual echoes), readout sample spacing $=4.5 \mathrm{~ms}$ and total acquisition time of 8:33 min. Dielectric pads containing a $25 \%$ suspension of barium titanate in deuterated water were placed proximal to the temporal lobe area to locally increase the transmit $\mathrm{B}_{1}{ }^{+}$field and to improve its homogeneity across the brain (Teeuwisse et al., 2012). To analyze within-subject reproducibility for each parameter, data were acquired twice for a subset of the subjects $(\mathrm{N}=3)$.

\subsection{2. | Data preparation}

The $\mathrm{T}_{2}{ }^{*}$ maps were obtained from the GRE data using a mono-exponential fit $\left(f(T E)=S_{0} e^{-T E / T_{2}{ }^{*}}\right.$ ), whereas the $\mathrm{T}_{2}{ }^{*} \mathrm{~W}$ image was calculated by dividing the image at $\mathrm{TE}_{4}$ by the image acquired at $\mathrm{TE}_{1}$ to correct for receive bias fields. The $\mathrm{T}_{1} \mathrm{w}$ maps used in this study are MP2RAGE images calculated from the two image volumes (INV1 and INV2) acquired at $\mathrm{TI}_{1}$ and $\mathrm{TI}_{2}$ (see sequence details), which minimizes the effect of $\mathrm{B}_{1}^{+}$ variations through space. A $T_{1}$ map was calculated online by linear interpolation of the INV1 and INV2 images (see Marques et al. (2010), for more details). Both the $T_{1} w$ and $T_{1}$ map were post-hoc corrected for variations in $\mathrm{B}_{1}{ }^{+}$using the same method as described in Marques and Gruetter (2013). All data were co-registered and resliced using a rigid-body transformation ( 6 degrees of freedom) and $7^{\text {th }}$ degree B-Spline interpolation to the $T_{1} w$ map to correct for subject motion between acquisitions using SPM 8 (Wellcome Department of Imaging Neuroscience, University College London, London, 


\section{CHAPTER 3}

UK). Quantitative $R_{1}$ and both ratio images $\left(T_{1} w / T_{2}{ }^{*} W\right.$ and $\left.R_{1} / T_{2}{ }^{*}\right)$ were generated using the mitools software package (http://od1n.sourceforge.net/). Note that we use $R_{1}$ instead of $T_{1}$ for comparison with the $T_{1} w$ images as the contrast is reversed between these two images (see Results section for details).

\subsection{3. | Processing pipeline}

Several data pre-processing steps were performed to prepare MP2RAGE data for FreeSurfer 5.3.0-HCP (http://surfer.nmr.mgh.harvard.edu/) processing (see Fig. 3.1 for illustration of the computational workflow). First, skull stripping was performed by obtaining a brain mask using the INV2 image. The INV2 image was used as it provides the best intra- and extra-cranial tissue contrast. Second, the generated brain mask was combined with a probability map of the dura mater for brain extraction. These initial steps were performed using MIPAV 7.1.1 (Center for Information Technology, NIH, Bethesda, USA), JIST 3.0 (Johns Hopkins University, Baltimore, USA) and CBS High-Res Brain Processing tools 3.0.5 (Max Planck Institute for Human Cognitive and Brain Sciences, Leipzig, Germany). Next, gradient distortion correction was applied on the brain extracted and other volumes using the gradient coefficients file provided by the scanner manufacture. This step is part of the Human Connectome Project (HCP) high-res analysis pipeline, which is especially designed to handle sub-millimeter resolution datasets (Glasser et al., 2013). The skull-stripped and gradient distortion corrected $\mathrm{T}_{1} \mathrm{w}$ volume was used as input to the HCP high-res analysis pipeline. As part of this pipeline, the $T_{1} w$ volume's resolution was downsampled to $1 \mathrm{~mm}$ isotropic resolution and used for FreeSurfer's recon-all method for cortical segmentation. Note, however, the final cortical surfaces were generated in the native resolution $0.7 \mathrm{~mm}$ isotropic). The resulting transformations matrices were also applied to the other parameter images for coregistration.

For each subject, all parameters were projected onto the surface using FreeSurfer's mri_vol2surf function both (i) by averaging between $20 \%$ and $80 \%$ of the cortical thickness to reduce potential partial voluming with WM and CSF and, alternatively, (ii) by sampling at specific relative cortical depths between 0\% (WM-GM border) and $100 \%$ (pial surface) of the cortical thickness with steps of 10\%, resulting in 11 depths fractions. All surface maps were coregistered to the 'fsaverage' subject for further analyses. To avoid any curvature-related changes in the spatial distribution of the cortical $T_{1} w$ or $R_{1}$ signal (Lutti et al., 2014), curvature-residualized maps of $T_{1} w$ and $R_{1}$ variation were computed for each subject in MATLAB (R2013B, The MathWorks, Natick, Massachusetts, USA). Final surface maps were averaged across subjects $(\mathrm{N}=10)$. 


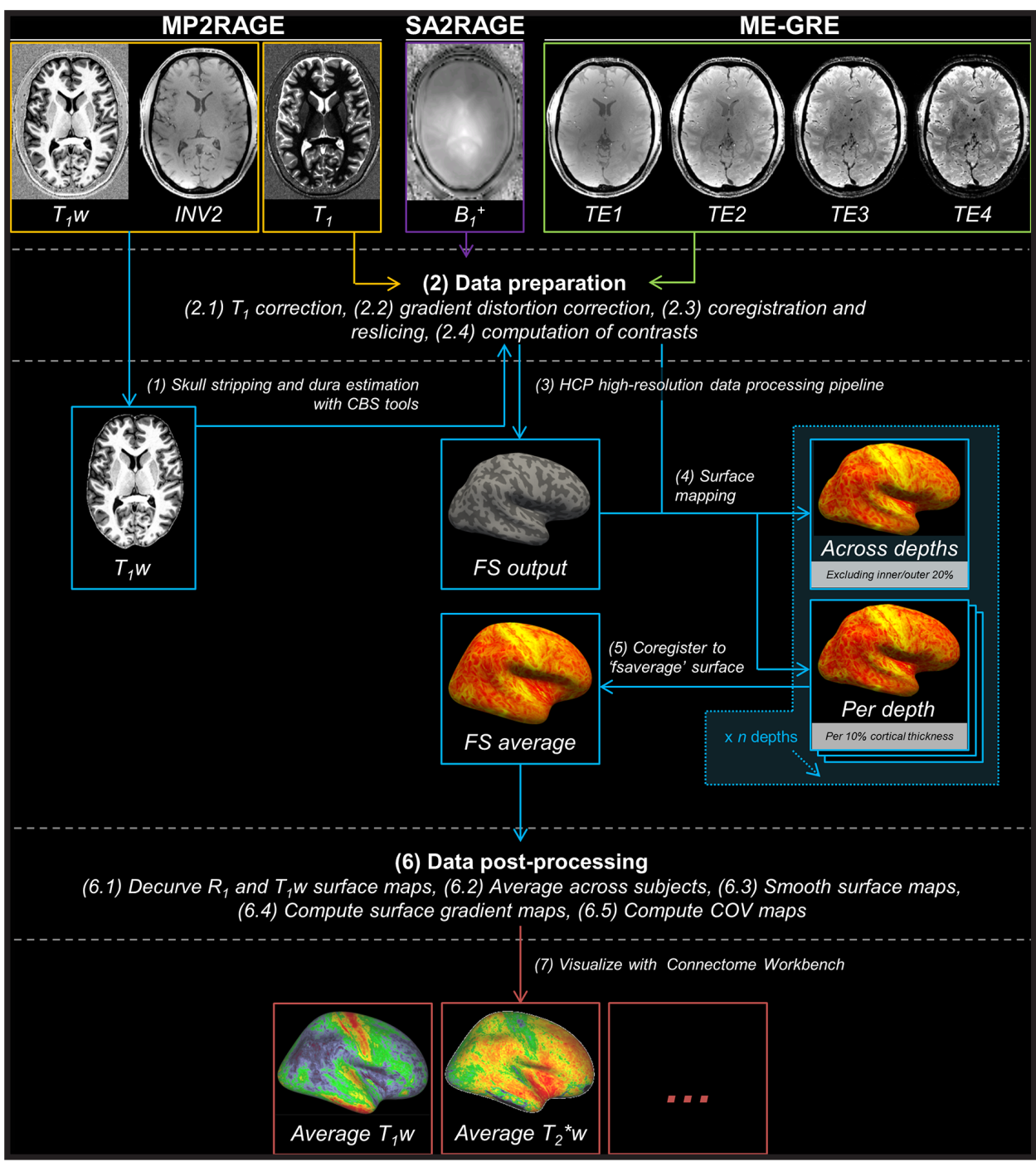

Fig. 3.1. The analysis pipeline for cortical myelin mapping using MP2RAGE (in orange), SA2RAGE (in purple) and ME-GRE (in green) data. MP2RAGE images are used for reconstruction of the cortex (in blue). Skull stripping (1), data preparation (2), HCP high-resolution data processing pipeline (3), surface mapping and cortical depth sampling (4), coregistration to 'fsaverage' surface (5), data post-processing (6) and visualization (7) are performed in this order.

\subsection{4. | Data visualization}

The surface maps were visualized using the Connectome Workbench v1.2.2 viewer (Washington University School of Medicine, Saint Louis, Missouri, USA) after conversion of the inflated surfaces and overlays to a compatible format. Non-cortical tissue in between hemispheres was masked using FreeSurfer's parcellation scheme to avoid 


\section{CHAPTER 3}

inappropriate scaling of the surface maps. A color map was chosen that optimally highlighted the contrast between lightly- and heavily-myelinated areas, respectively.

\subsection{5. | Regions of interests}

Several regions of interests (ROIs, see Supplementary Fig. 3.1) were selected from the PALS-B12 atlas and FreeSurfer's parcellation for a more detailed comparison between MR parameters and included heavily-myelinated regions (probabilistic Brodmann areas [BAs] 1, 2, 3, 4, 17, 18, MT and transverse temporal gyrus [gTT] and sulcus [sTT]), moderately myelinated regions (BAs 6, 44 and 45) and a lightly myelinated region (BA29, i.e. retrosplenial cortex).

\subsubsection{Quantitative data analysis}

Quantitative comparison between parameters was done for multiple parameters obtained using custom scripts written in MATLAB. First, the inter-subject $(N=10)$ and intra-subject $(\mathrm{N}=3$ ) coefficients of variation (COV) were computed using the vertex data from the averaged surface map. The inter-subject COV estimates the effects of myelination variability across subjects on each parameter, while the intra-subject COV assesses the scan-rescan reproducibility within subjects. In both cases, the COV was computed for each vertex.

$$
C O V_{\mathrm{pv}}=\frac{\sigma_{\mathrm{pv}}}{\mu_{\mathrm{pv}}} \frac{1}{F W H M}
$$

Inter-subject and intra-subject COVs ( \pm inter-vertex standard error, i.e. SE) were calculated by dividing the standard deviation $(\sigma)$ for each vertex $(v)$ and parameter $(p)$ by the mean value $(\mu)$ of that same vertex across subjects or time points, respectively. These values were than normalized to the full-width-half-maximum (FWHM), of the average cortical distribution (after rescaling the values between the $3^{\text {rd }}$ and $97^{\text {th }}$ percentiles to range between 0 and 1) for each parameter to take into account differences in the distribution of the values (see also the Discussion section).

Second, we computed the parcellability variation (PV) for each vertex to determine how reliably a vertex value can be located within the cortical histograms and therefore how reliably it might be parcellated. The PV was calculated as follows:

$$
P V_{\mathrm{pv}}=\frac{\sigma_{\mathrm{pv}}}{F W H M_{\mathrm{p}}}
$$


The PV was calculated by dividing the average (across subjects) scan-rescan standard deviation $(\sigma)$ for each vertex $(v)$ and parameter $(p)$ by the FWHM of the average cortical distribution (before rescaling) and averaged for each region ( \pm inter-vertex SE). As a result, a lower PV indicates a higher parcellability of the corresponding region.

Third, to determine the quality of the surface maps based on the contrast between heavily- and lightly-myelinated regions, the contrast-to-noise ratio (CNR) per unit of time was calculated vertex-wise for each region and parameter similar to Deistung et al. (2013):

$$
C N R_{\mathrm{pyv}}=\frac{\left|s_{\mathrm{pyv}}-s_{\mathrm{psv}}\right|}{\sigma_{\mathrm{s}+\mathrm{y}}} \frac{1}{\sqrt{t}} \text { per unit time }(\mathrm{min}) .
$$

CNR for each MRI parameter ( $p$ ) was computed by dividing the absolute differences between the intensity of a vertex $(v)$ in an $\mathrm{ROI}(\mathrm{y})$ and a randomly selected vertex value in a lightly-myelinated (defined by its average value) neighboring region (s) by the inter-vertex SD of the signal intensities of all the pooled vertices from both regions ( $\sigma$ ). The latter value served as an estimate of the noise. Different less myelinated regions proximal to the respective $\mathrm{ROI}$ were used to minimize the effects of possible residual transmit/receive bias fields on the observed inter-region contrast. Mean CNR ( \pm inter-vertex SE) was calculated across all vertices for each parameter and region and converted to CNR per unit time, by normalizing it to the square root of $t$ (minutes).

\subsection{7. | Vertex-wise correlation between parameters}

The vertex-wise correlation (Pearson correlation $r$ ) between $T_{1} w$ and $T_{2} w, R_{1}$ and $T_{2}{ }^{*}$, $R_{1} / T_{2}^{*}$ and $T_{1} w / T_{2}^{*} W$ was assessed to globally investigate their relationship. This was done on the data averaged across depths but also, separately, as a function of cortical depths. Vertices clearly affected by susceptibility differences due to $B_{0}$-inhomogeinities and characterized by an average $\mathrm{T}_{2}{ }^{*}$ lower than $0.024 \mathrm{sec}$ were excluded. For reference, average $\mathrm{T}_{2}{ }^{*}$ value observed in the white matter of a single subject was approximately $0.027 \mathrm{sec}$.

\subsection{8. | Cortical surface pattern analysis}

Surface pattern analysis was performed to qualitatively compare between parameters. Here, we focused on the heavily-myelinated regions close to the central sulcus and auditory cortex and compared the area size and location of these regions between the parameters, based on the group surface maps after pre-smoothing the data with a geodesic Gaussian kernel of $1 \mathrm{~mm}$, using two approaches to define areal borders. 


\section{CHAPTER 3}

First, we compared the parameters based on threshold contours. Thresholds (\%) were based on the area under the curve (AUC) of the global histogram between the $3^{\text {rd }}$ and $97^{\text {th }}$ percentile. However, as cortical myelin concentration is a continuous variable, there is no precise threshold value to determine cortical area borders. To determine the "subjectivity" (i.e. threshold dependency), we analyzed the area covered (\% of vertices within specific ROIs) by quantifying the portion of vertices with intensity values equal to or higher than that defined by several thresholds, ranging from $60 \%$ to $90 \%$ of the AUC of the cortical histograms. Note, that, for this purpose only, we transformed the $T_{2}^{*}(w)$ data to $R_{2}^{*}(w)$, so that its profile across thresholds matches with those from the other parameters. Three thresholds (75\%, $80 \%$ and $85 \%$ of AUC) were eventually selected for visualization on the surface and quantification of overlap of areas between parameters (see Supplementary Material 1).

Alternatively, areas borders can be determined using the gradient of the parameters (Glasser and Van Essen, 2011; Glasser et al., 2016). For this, the pre-smoothed group surface maps were used to compute local maximum gradients using the 'metric-gradients function' within the Connectome Workbench command. In brief, at each vertex, the gradient is computed using a regression between the values of the vertex and of its neighboring vertices after spatially transforming them into a plane tangent. The gradient is then given by the slopes of the regression and reconstructed as a surface overlay.

\section{3. | RESULTS}

\subsection{1. | Comparing myelin-related cortical patterns using different contrasts at 7T}

In general, similar spatial myelin-related patterns are observed for all different parameters obtained (see Fig. 3.2 for the single-subject and group data). For simplicity, only the right hemispheres are shown as there were no evident differences between the left and right hemispheres. The values for the primary areas (including the somatosensory, motor, auditory and visual cortex) deviate most from the average values, which is in accordance with previous studies that showed strong myelination/increased cortical contrast in these areas at 1.5T, 3T and 7T using different MR parameters (Sigalovsky et al., 2006; Glasser and Van Essen, 2011; Cohen-Adad et al., 2012; Bock et al., 2013; Sereno et al., 2013; De Martino et al., 2014; Lutti et al., 2014).

In all subjects $(\mathrm{N}=10)$ and for all parameters, most dominant myelination-weighted contrast is observed along the posterior (S1, i.e. BA2 and BA3) and anterior (M1, i.e. BA4) cortices around the central sulcus (CS). This area extends from the paracentral 


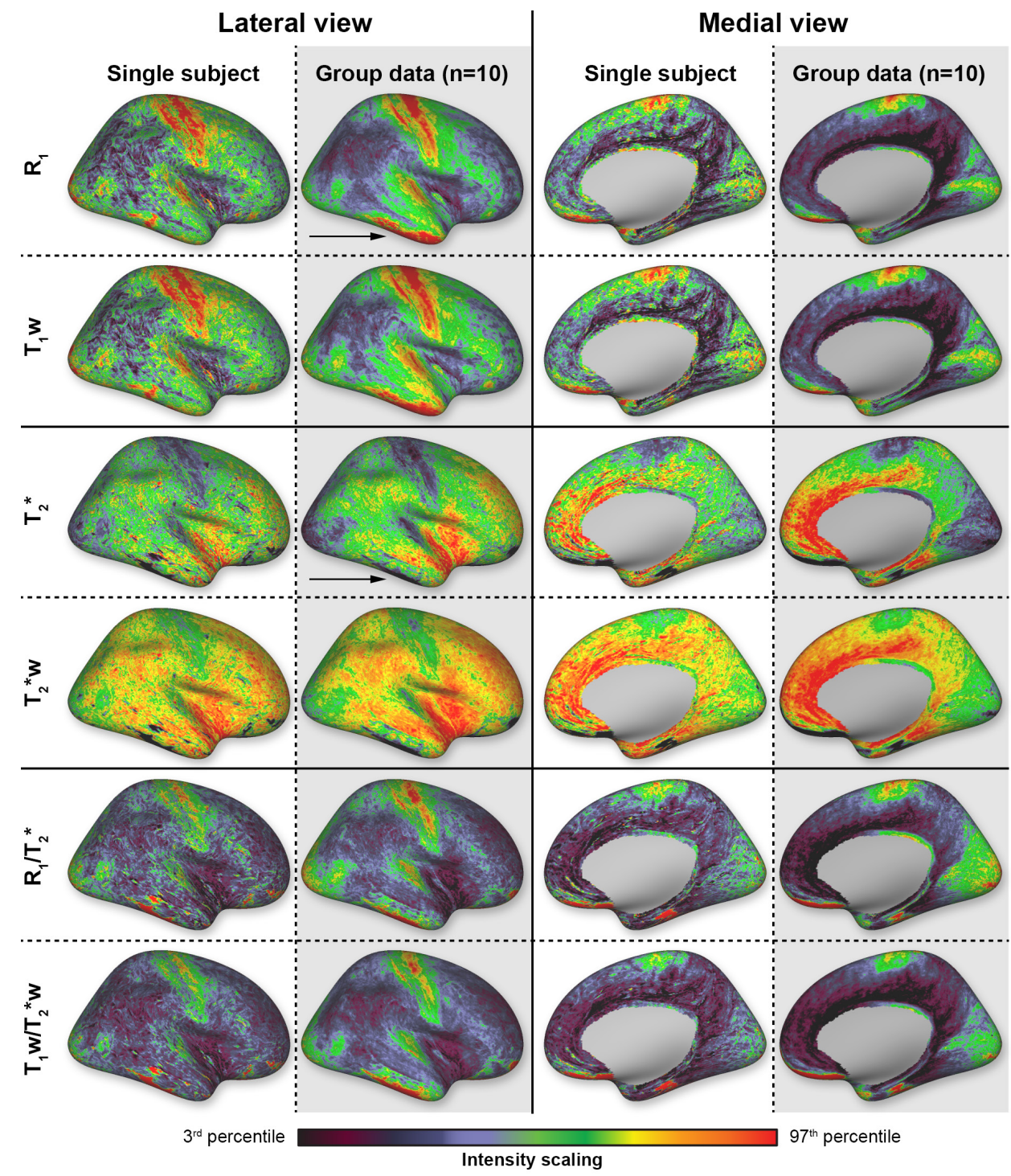

Fig. 3.2. | Overview of the different parameters (rows) mapped on the reconstructed cortical surface. Data are shown from both lateral and medial perspectives for a single-subject (white columns) and the average brain ( $N=10$, gray columns). Scaling is based on the $3^{\text {rd }}$ and $97^{\text {th }}$ percentiles.

lobule towards the lateral sulcus. Increased contrast levels are also observed beyond the lateral sulcus in the superior temporal gyrus and in particular Heschl's gyrus (HG), encompassing the auditory cortex (AC). More posteriorly, towards the occipital lobe, increased myelination is also observed close to the middle temporal (MT) region. Other regions of the visual system located on the medial side are also characterized by increased contrast levels and cover almost entirely the medial occipital lobe (including 


\section{CHAPTER 3}

V1 and V2). High levels of myelination are also observed in the posterior part of the cingulate cortex. Lower levels of myelination are seen in the frontal regions and the middle and inferior temporal gyri. This contrast, between areas with higher and lower myelination, is enhanced after calculating the ratio images for both the weighted and quantitative $T_{1}$ and $T_{2}{ }^{*}$ images (lower two rows in Fig. 3.2). Clear artifacts due to $B_{1}^{+}$ and $\mathrm{B}_{0}$-inhomogeinities can be seen in the inferior temporal and frontal lobes (pointed out by black arrows in Fig. 3.2) and these regions were, therefore, excluded from the quantitative analyses below.

A

Quantitative
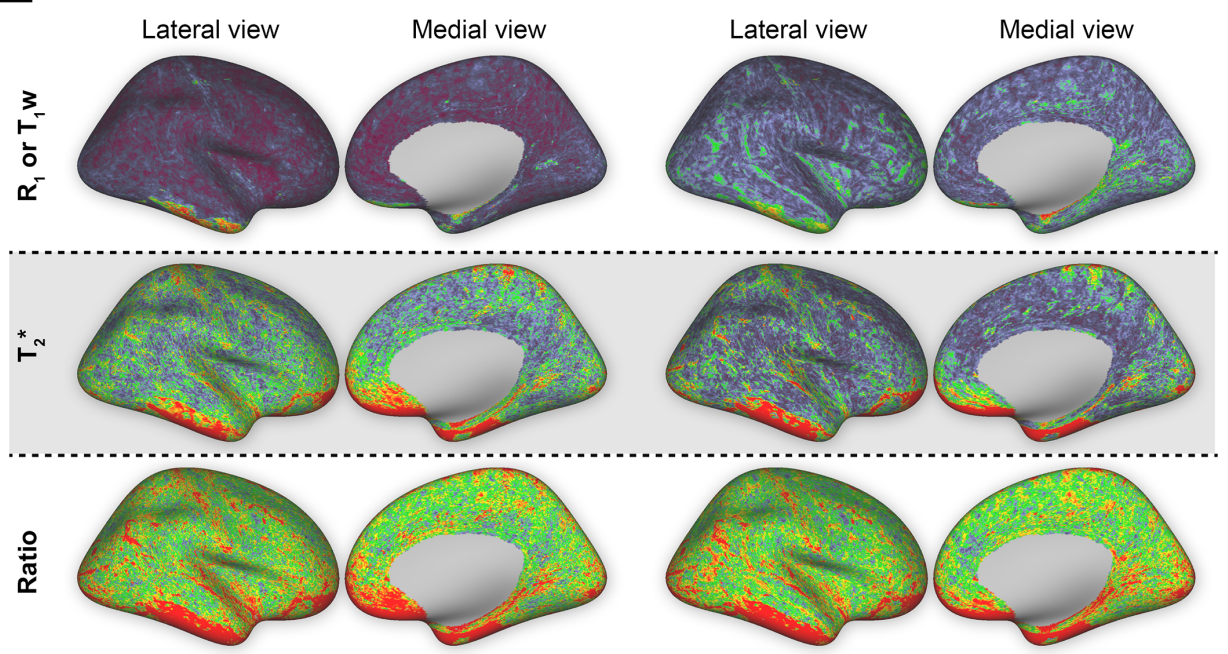

Relative inter-subject coefficient of varation (COV)
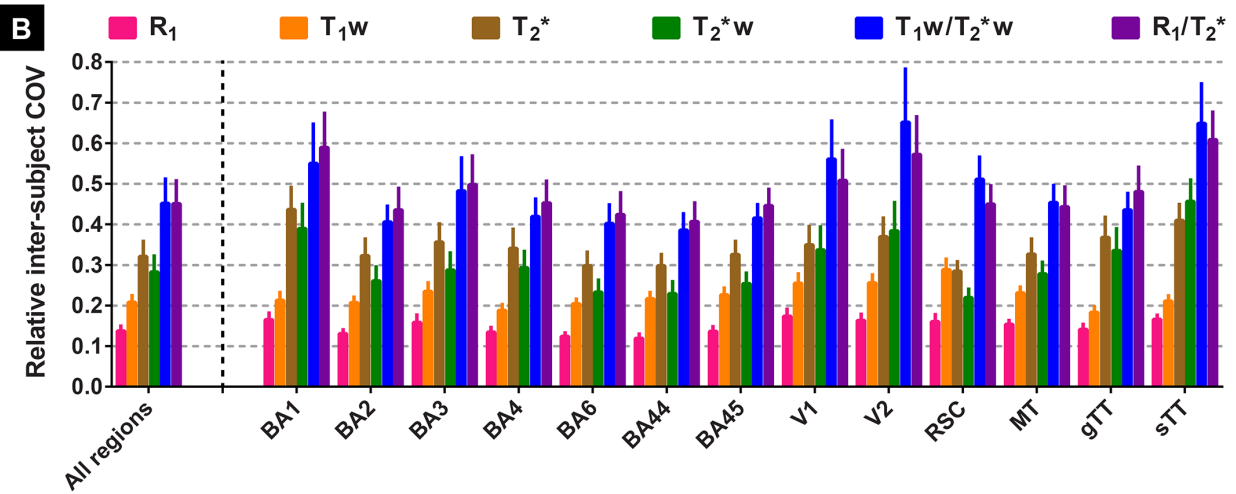

Fig. 3.3. | Average relative inter-subject coefficients of variation (COV) comparison between the parameters investigated for the whole cortex, the selected regions of interest (ROIs) and all ROIs combined. Relative inter-subject whole-brain COV maps are shown in A. Mean values ( \pm inter-vertex $S E$ ) of inter-subject COV were extracted for each ROI (see Supplementary Fig. 3.1) from the COV maps in $A$ and plotted in $B$. 


\subsection{2. | Inter- and intra-subject variability}

The relative inter-subjects $(\mathrm{N}=10)$ and intra-subjects $(\mathrm{N}=3)$ COV was determined to compare the different parameters in terms of similarity across healthy subjects and reproducibility within-subjects, respectively. Fig. 3.3A shows the relative (i.e. normalized) inter-subject COV across the entire cortex. One can observe the lowest inter-subject COV for $\mathrm{R}_{1}$ (top row, left column) compared to the remaining parameters. Highest inter-subject COV can be seen for the ratio images (bottom row). The same trend is evident when analyzing the cortical ROIs individually (see Fig. 3.3B): lowest COV for

A

Quantitative

Weighted
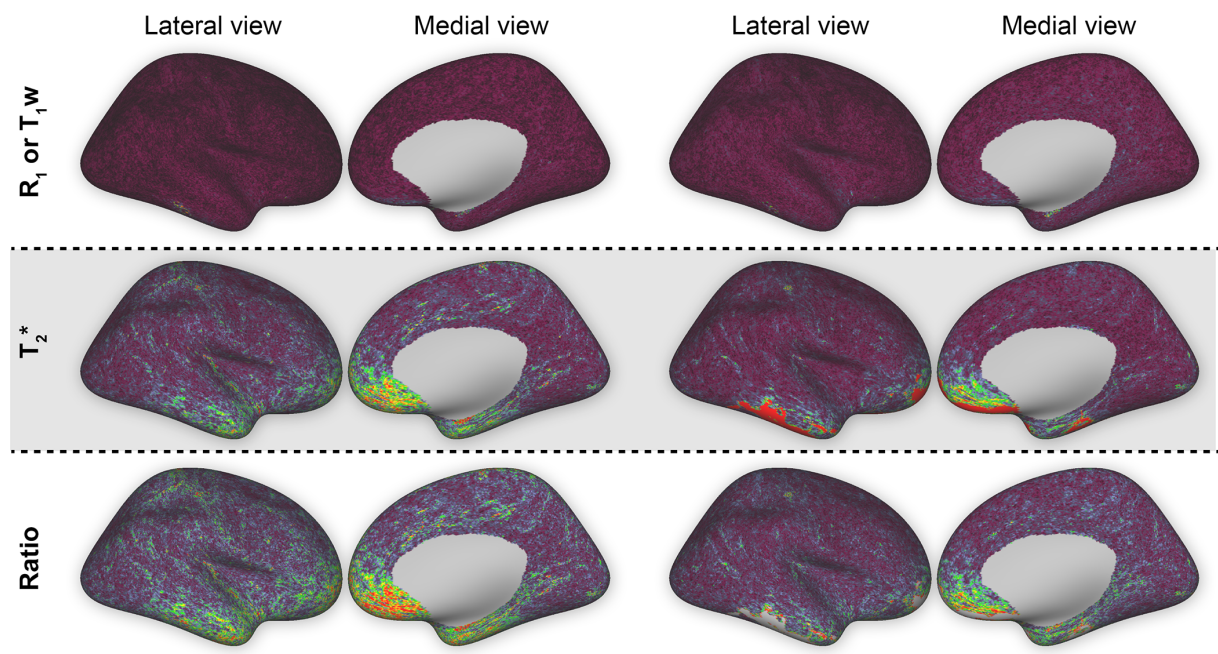

$>0.7$

Relative intra-subject coefficient of varation (COV)

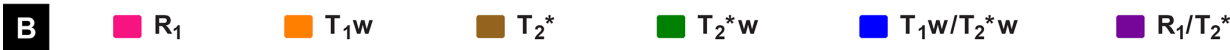

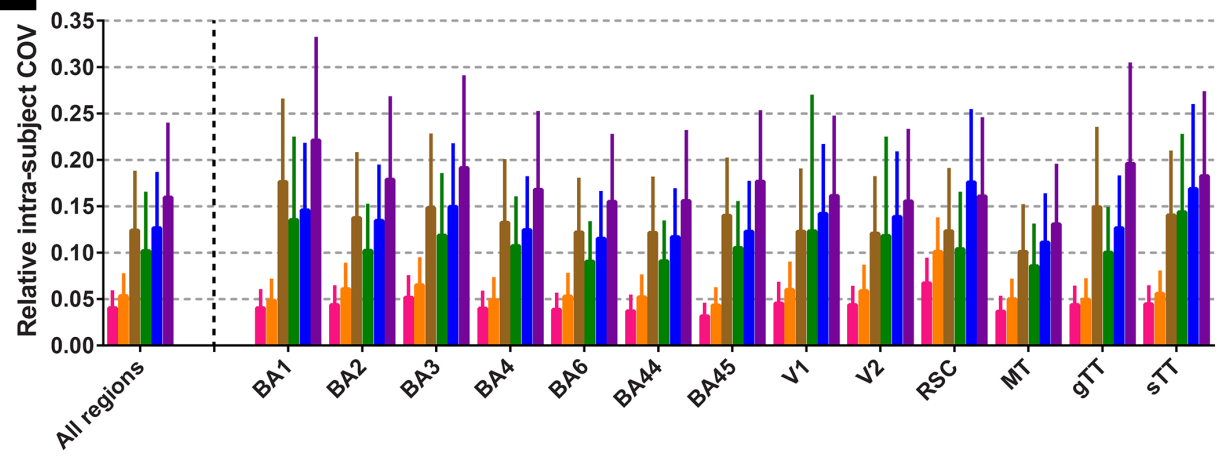

Fig. 3.4. Average relative intra-subject coefficients of variation (COV) comparison between the parameters investigated for the whole cortex, the selected regions of interest (ROls) and all ROls combined. Relative intra-subject whole-brain COV maps are shown in A. Mean values ( \pm inter-vertex SE) of intra-subject COV were extracted for each ROI (see Supplementary Fig. 3.1) from the COV maps in $A$ and plotted in $B$. 


\section{CHAPTER 3}

$R_{1}\left(0.137 \pm 0.012\right.$, averaged across regions) and highest for the quantitative $T_{2}{ }^{*}$ and weighted ratio images $(0.451 \pm 0.057$ and $0.451 \pm 0.060$, respectively). A similar pattern is observed for the non-normalized COV values (Supplementary Fig. 3.2). However, normalization especially corrects for the difference between $\mathrm{T}_{2}{ }^{*}$ and $\mathrm{T}_{2}{ }^{*} \mathrm{~W}$ parameters (see Table 3.1 for an overview of the FWHMs used for normalization).

Table. 3.1 | Overview of the FWHMs used to normalize the COV and to compute parcellability for each parameter.

\begin{tabular}{l|l|l} 
Parameter & FWHM (after rescaling) & \multicolumn{1}{l}{ FWHM (before rescaling) } \\
$\mathbf{R}_{\mathbf{1}}$ & 0.4026 & $0.0503 \mathrm{sec}-1$ \\
\hline $\mathbf{T}_{\mathbf{2}}{ }^{*}$ & 0.4788 & $0.0083 \mathrm{sec}$ \\
\hline $\mathbf{R}_{\mathbf{1}} / \mathbf{T}_{\mathbf{2}}{ }^{*}$ & 0.3888 & $4.4240 \mathrm{sec}-2$ \\
\hline $\mathbf{T}_{\mathbf{1}} \mathbf{w}$ & 0.4573 & 339.0132 a.u. \\
\hline $\mathbf{T}_{\mathbf{2}}{ }^{*} \mathbf{W}$ & 0.3276 & 0.0764 a.u \\
\hline $\mathbf{T}_{\mathbf{1}} \mathbf{w} / \mathbf{T}_{\mathbf{2}}{ }^{*} \mathbf{W}$ & 0.3313 & 861.9166 a.u. \\
\hline
\end{tabular}

The intra-subject COV reveals that $\mathrm{R}_{1}$ is outperforming all other parameters in terms of reproducibility when comparing multiple images of the same modality acquired at different time points (see Fig. 3.4). In addition, spatial intra-subject COV maps reveal lower variability for the $T_{1} w$ compared to the remaining parameters.

\subsection{3. | Parcellability variation}

To compare the reliability of cortical parcellations based on the surface maps, the PV was computed for each parameter by dividing the vertex-specific intra-subject standard deviation (averaged across subjects, $\mathrm{N}=3$ ) by the FWHM of the corresponding global histogram (see Table 3.1 for an overview of the FWHMs). Region-specific averages ( \pm inter-vertex $\mathrm{SE}$ ) and all regions together are plotted in Fig. 3.5. In general

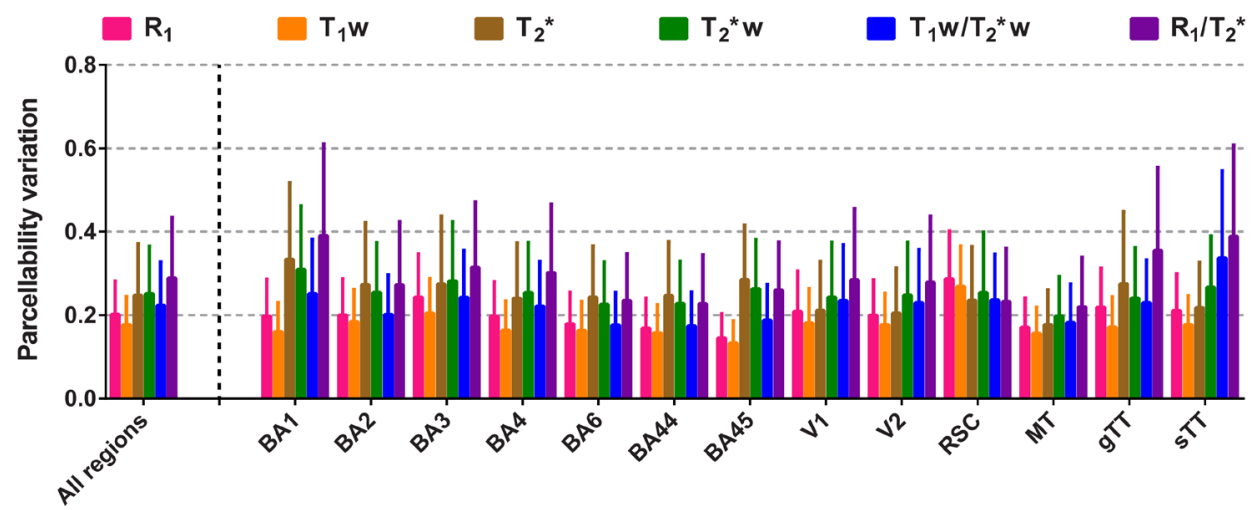

Fig. 3.5. | Comparison of the parcellability variation (PV) between the parameters. Mean PV values ( \pm inter-vertex SE) are plotted for each parameter, region of interest (ROI) and all ROIs combined. 
(except for the RSC), $R_{1}$ and $T_{1} w$ have the lowest (and comparable) PV, weighted against the other parameters. $\mathrm{T}_{2}{ }^{*}$ and $\mathrm{T}_{2}{ }^{*} \mathrm{~W}$ are similar to each other, while highest scores were observed for the quantitative ratio.

\subsection{4. | Contrast-to-noise comparison}

Fig. 3.6 shows the CNR per unit time (minute, mean \pm intra-vertex $\mathrm{SE}$ ) and per parameter for each of the different regions and all regions together. All parameters have comparable CNR per unit time. As for parcellability, the longitudinal relaxation constants $\left(R_{1}\right.$ and $\left.T_{1} w\right)$ outperform the transversal ones $\left(T_{2}{ }^{*}\right.$ and $\left.T_{2}{ }^{*} \mathrm{~W}\right)$ by having slightly higher CNR. When the ROls are considered individually, CNR is lowest in the lightly-myelinated regions $\mathrm{BA} 6, \mathrm{BA} 44, \mathrm{BA} 45$ and $\mathrm{RSC}$ and highest in the heavily-myelinated regions like BA2, BA3, BA4, V1, V2, AC (sTT and gTT) and MT for all parameters. $\mathrm{R}_{1}$ and $\mathrm{T}_{1}$ w show slightly higher CNRs for all heavy-myelinated regions, with the exception of MT where they score slightly lower.

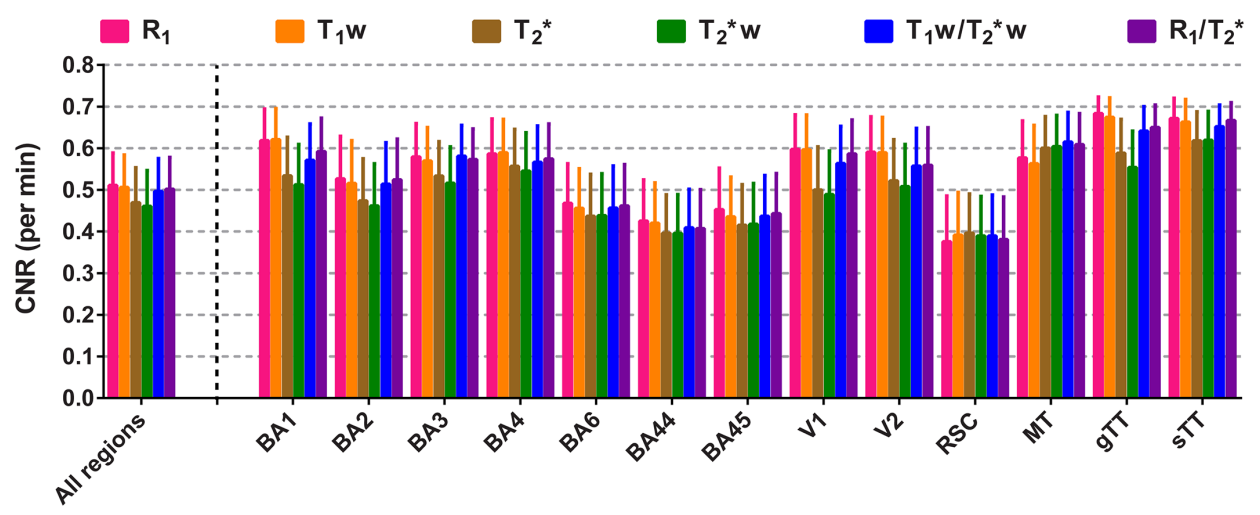

Fig. 3.6. | Comparison of the mean contrast-to-noise ratio (CNR) per scanning time (in minutes). Mean values of CNR per minute ( \pm inter-vertex SE) are plotted for each parameter, region of interest (ROI) and all ROls combined.

\subsection{5. | Vertex-wise correlation between contrasts}

Fig. 3.7A shows the linear fits between the (i) $R_{1}$ and $T_{2}{ }^{*}$ values (Pearson's $r=-0.38$ ), (ii) $T_{1} w$ and $T_{2}{ }^{*} W$ values $(r=-0.36)$ and (iii) $R_{1} / T_{2}{ }^{*}$ and $T_{1} w / T_{2}{ }^{*} W(r=0.88)$, across all vertices. In all three cases, highest correlations are observed between the WM/GM border and mid-thickness level, but the correlation decreases strong when moving towards the GM/CSF border for $\mathrm{R}_{1}$ (or $\mathrm{T}_{1} \mathrm{w}$ ) vs $\mathrm{T}_{2}{ }^{*}$ (or $\mathrm{T}_{2}{ }^{*} \mathrm{~W}$, see Fig. 3.7B). 
A

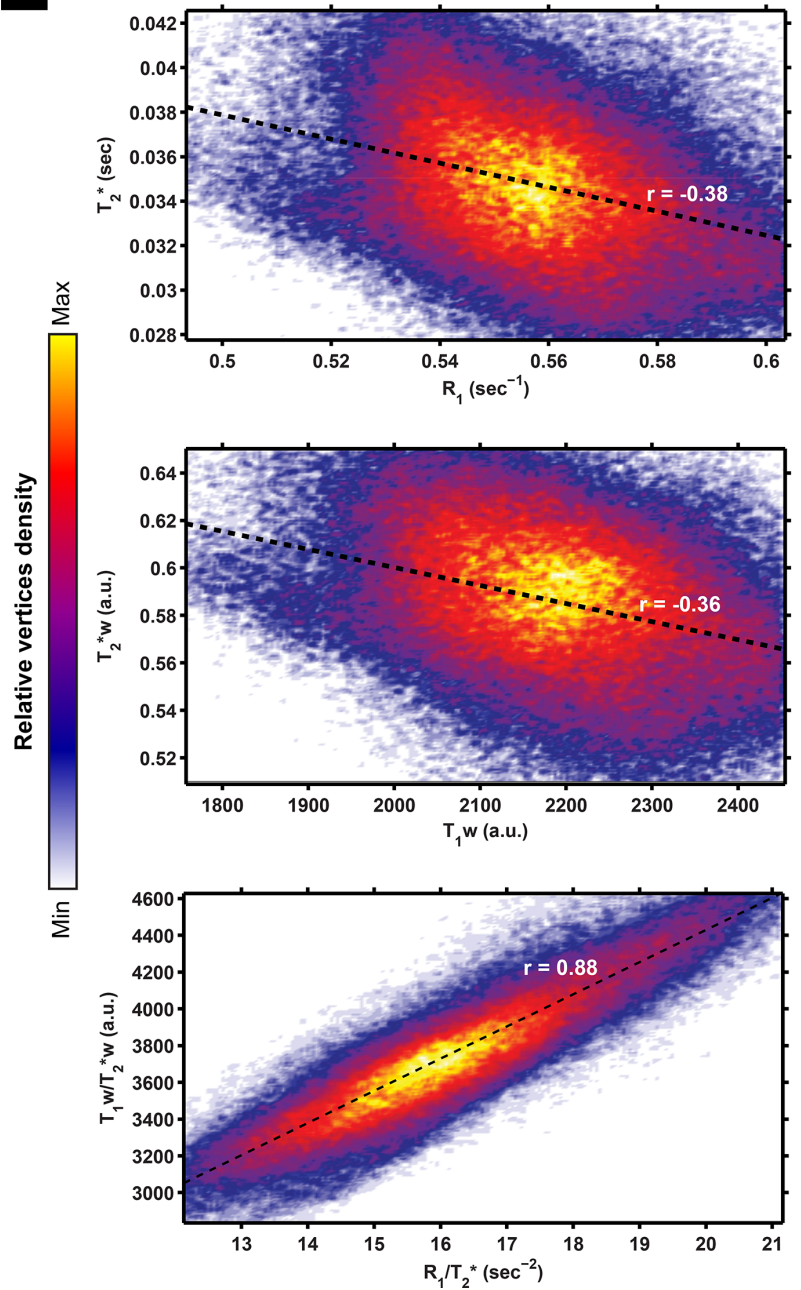

B

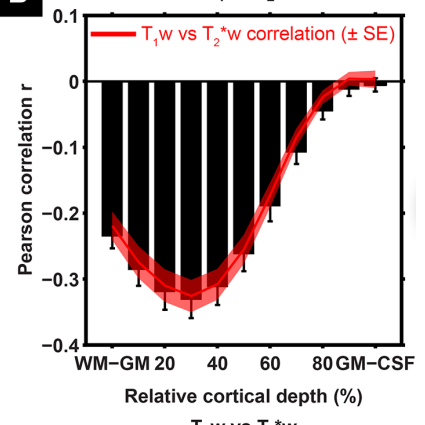

$\mathrm{T}_{1} \mathrm{w}$ vs $\mathrm{T}_{2}{ }^{*} \mathrm{w}$

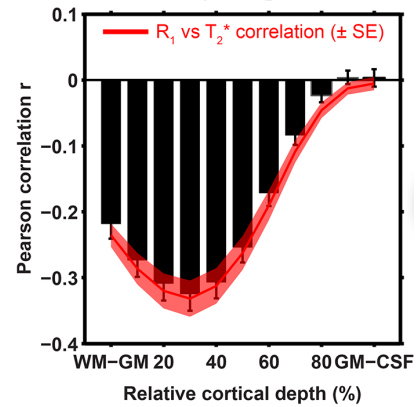

$\mathrm{R}_{1} / \mathrm{T}_{2}{ }^{*} \mathrm{vs} \mathrm{T}_{1} \mathrm{w} / \mathrm{T}_{2}{ }^{*} \mathrm{w}$

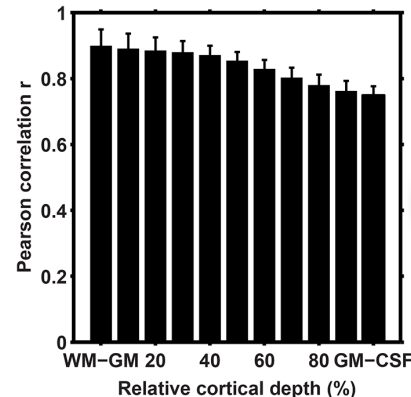

Relative cortical depth (\%)

Fig. 3.7. | Surface-based comparison of the correlations between $R 1$ (or $T_{1} w$ ) and $T_{2}^{*}$ for both the quantitative (top row), weighted (middle row) as well as the ratio (bottom row) parameters. For each pair of parameters, linear fits (black dashed line) were computed after plotting of the $R_{1}$ (or $T_{1} w ; x$-axis in $A)$ and $T_{2}{ }^{*}(y$-axis in $A)$ value of each vertex, where the color is determined by the relative vertices density. Pearson correlation $r$ between both datasets was computed and plotted as a function of cortical depth (B, mean \pm inter-subject SE).

\subsection{6. | Threshold dependency analysis}

Fig. 3.8 displays the portion of vertices with intensity values equal to or higher than that defined by several thresholds ( $x$-axes) for each parameter (colored solid lines) and strongly-myelinated region. We focused on thresholds between $60 \%$ and $90 \%$ of AUC, as lower and higher thresholds are not relevant for our purpose since the transition between lightly- and heavy-myelinated regions lies within this range. The 
Fig. 3.8. |Observer-independence analyses of the threshold contours approach. For each parameter and different heavily-myelinated regions (central sulcus [including M1 and S1], auditory cortex, visual cortex [including V1 and V2] and MT), the percentage of vertices (of the total region of interest) are plotted as a function of threshold level (60-90\% based on the AUC of the corresponding histogram). Vertical dashed lines indicate the three thresholds used in Fig. 3.8.

profiles revealed that $T_{1} w$ and $\mathrm{R}_{1}$ cover the largest surface areas with respect to $C S$ and $A C$, independent of the threshold value, whereas $\mathrm{T}_{2}{ }^{*}(\mathrm{w})$ cover the smallest surface areas. In contrast, this pattern is opposite for the visual cortex and MT. Compared to $\mathrm{R}_{1}$ and $\mathrm{T}_{1} \mathrm{w}$, a slightly larger variability of the slope within the visual cortex and MT was observed for the $T_{2}^{*}(w)$ and both ratio parameters. In general, the profiles of each parameter and region suggest that the differences between each parameter are relatively independent of the threshold.

\subsection{7. | Surface pattern comparison}

Here, we explored two approaches to delineate cortical areas based on their myelin content: using visual inspection of the location of (i) contours
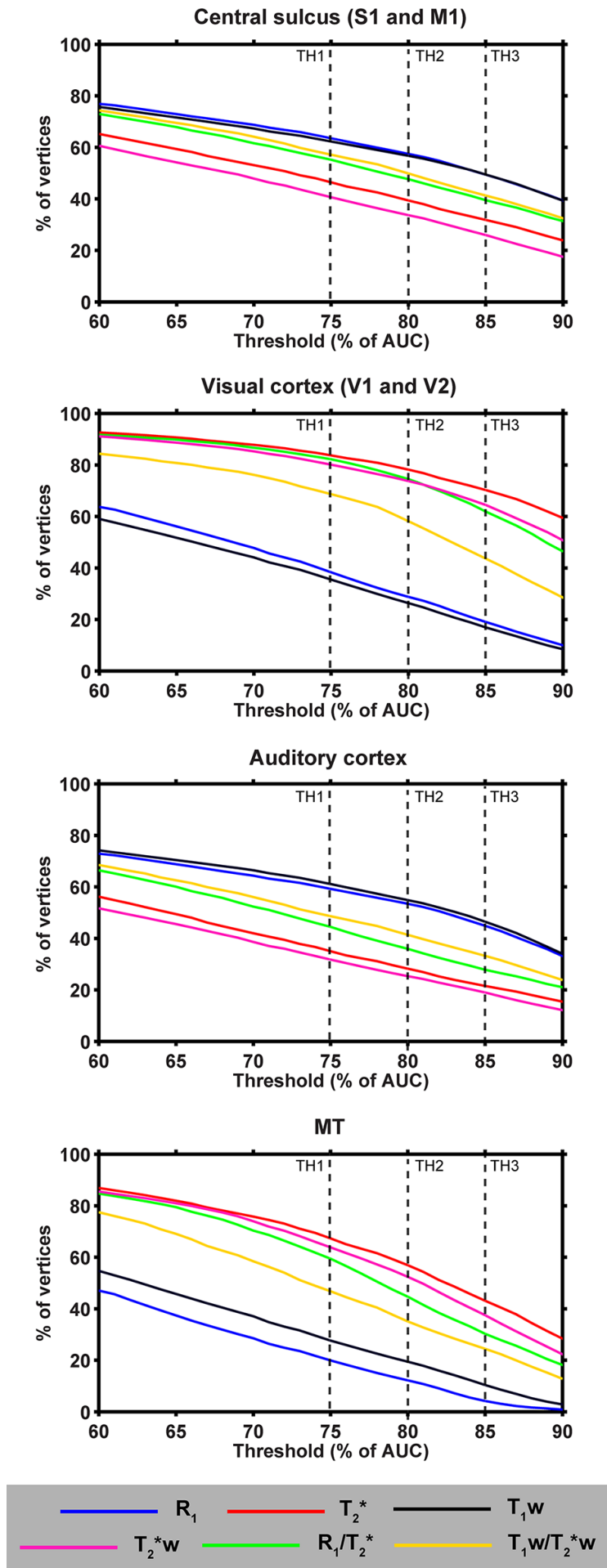


\section{CHAPTER 3}

based on thresholds and (ii) strong intensity gradients on the surface relative to a specific delineation of regions. Fig. 3.9 shows a comparison between the quantitative parameters (see Fig 3.9A) using contours based on three threshold levels (red, blue and light-blue solid lines in Fig. 3.9B). Based on visual inspection, the delineated areas around the $C S$ and $A C$ are largest for $R_{1}$ and smallest for $T_{2}{ }^{*}$, independent of the threshold. These observations are confirmed by quantitative analysis as shown in the Supplementary Fig. 3.3, where we focused on the total area of several regions $\left(\mathrm{mm}^{2}\right)$ and differences between parameters (using the Jaccard coefficient as a metric to define overlap), including those in other regions (i.e. MT and visual cortex). Moreover, we observe a shift in the location of $A C$ more posteriorly in the $\mathrm{T}_{2}{ }^{*}$ parameter (see Fig. 3.9 , red arrow) compared to $\mathrm{R}_{1}$ (white arrow).

In addition to the threshold approach, surface maps were compared between different parameters by computing the local maximum gradients. The surface maps in Fig. 3.9C emphasize the areal borders of different regions (especially BA1, BA4 and AC) based on the presence of strong intensity gradients across the surface for the different parameters. However, the gradient magnitude of these borders varies between parameters. For example, when comparing the magnitudes of the gradients among parameters, lower gradients are visible between BA4 and surrounding regions for $\mathrm{T}_{2}{ }^{*}$. Clearer surface gradients are visible for $T_{2}{ }^{*}$ and especially the $R_{1} / T_{2}{ }^{*}$ ratio when focusing on the posterior border of the AC. Overall, the borders that can be extracted from this data are not unambiguous and require user input (i.e. a choice of the gradient threshold value and filling-in gaps in the borders). Additional smoothing does not remove this ambiguity of the gradients and decreases the structural detail (see Supplementary Fig. 3.4). Based on these gradient maps, no clear variation, however, is observed with respect to the spatial location of regions between parameters, as was observed for the threshold contours approach.

\section{4. | DISCUSSION}

MRI has been used in the last three decades to visualize brain function, metabolites, vasculature, white and gray matter anatomy. In particular, $\mathrm{T}_{1}$-contrast MRI has been utilized to segment cortical gray and white matter. Recently, it has been proposed that $\mathrm{T}_{1}$ - and $\mathrm{T}_{2}{ }^{(*)}$-weighted $\mathrm{MRI}$ can also be employed to map myelin not only in white matter but also within the cortex, which can be used to parcellate the brain and to delineate brain areas (Sigalovsky et al., 2006; Geyer et al., 2011; Glasser and Van Essen, 2011; Cohen-Adad et al., 2012; Dick et al., 2012; Bock et al., 2013; Sereno et al., 2013; De Martino et al., 2014; Lutti et al., 2014; Tardif et al., 2015; Glasser et al., 2016). However, 
A

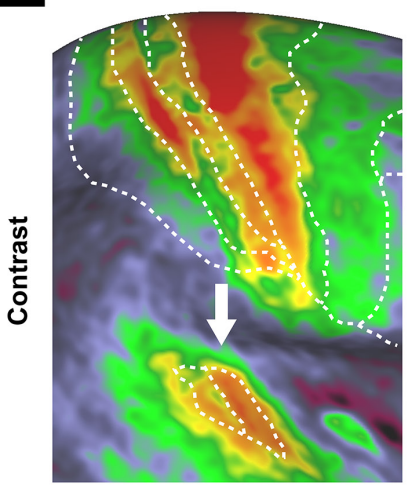

B

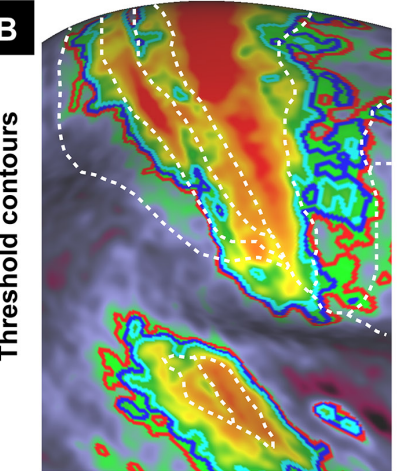

c

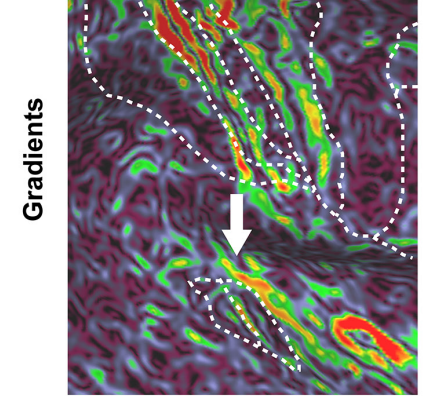

$\mathrm{T}_{2}{ }^{*}$
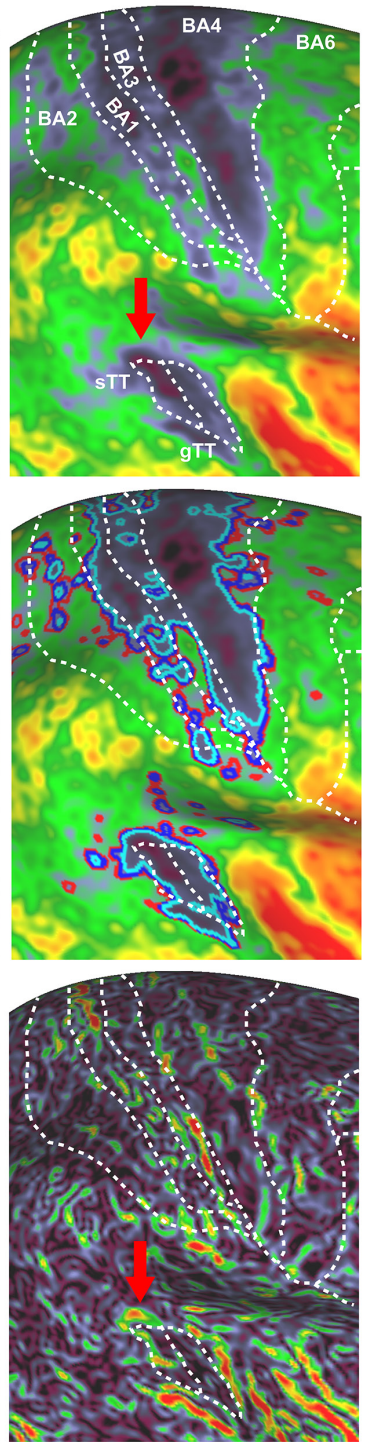

Ratio
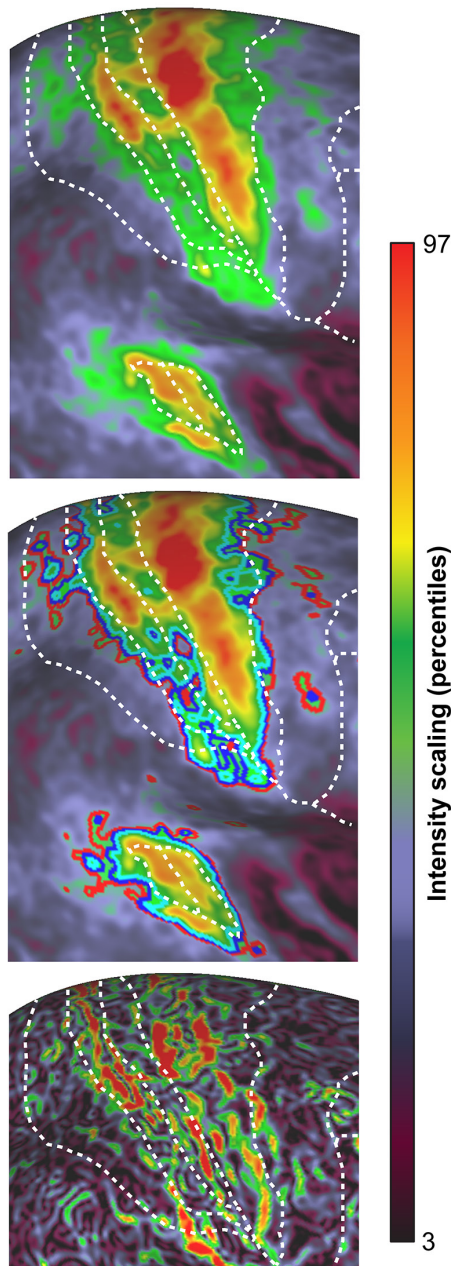

Threshold 1

Threshold 2

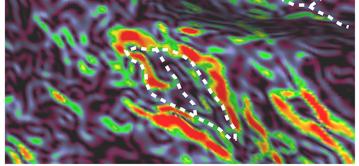

Fig. 3.9. | Surface pattern comparison across $R_{1} T_{2}^{*}$ and their ratio, based on threshold contours and surface gradients. To allow comparison between parameters, the boundaries of the regions of interest (ROIs) are superimposed. Supplementary Fig. 3.4 shows the effect of applying different pre-smoothing kernel sizes on the group-average surface maps and subsequent gradient maps.

no systematic comparison between the proposed approaches (i.e. $\mathrm{T}_{1}-\mathrm{T}_{2}{ }^{*}$-weighted images and their ratios as well as their corresponding quantitative MR parameters) has been performed so far. The goal of the current study is to fill this gap by evaluating the 


\section{CHAPTER 3}

previously proposed in vivo myelin-mapping approaches. To achieve this, we acquired high-resolution multi-parameter imaging data ( $\mathrm{T}_{1}$ and $\mathrm{T}_{2}{ }^{*}$, weighted and quantitative) using 7T and performed cortical surface-based analyses to quantitatively compare the inter-subject variability, intra-subject reproducibility and CNR between parameters. In addition, we analyzed the correlation between $T_{1}$ and $T_{2}{ }^{*}$ and their ratios and evaluated thresholding- and gradient contour-based approaches to delineate areal borders for cortical parcellation.

\subsection{1. | Inter- and intra-subject reproducibility}

One important quantitative parameter to compare different parameters is the COV (i.e. the extent of variability in relation to the mean of the distribution), which can be used to evaluate the inter-subject variation and intra-subject reproducibility for each parameter. Since the basic biochemical parameters (such as myelin density) of tissue should not be significantly affected within the time frame of the different acquisitions, the intra-subject COV is indicative of non-biochemical variability in the image contrast. Note that unlike to the conventional method of calculating the COV (i.e. $\sigma / \mu$ ), an additional normalization step was performed in the current study to take into account differences in the distribution of the values (within the $3^{\text {rd }}$ and $97^{\text {th }}$ percentiles) using the FWHM. For example, in the extreme case for which all absolute values of a parameter are projected into a single value in a weighted image, there is decreased sensitivity for myelin using the weighted approach. Thus, in comparing the reliability of quantitative and weighted MRI approaches (or different variants of weighted approaches), we propose that FWHM normalization of the distribution is performed for the COV comparison.

After normalization, the lowest inter- and intra-subject COVs are generally observed for $\mathrm{R}_{1}$, followed by $\mathrm{T}_{1} \mathrm{~W}, \mathrm{~T}_{2}{ }^{*} \mathrm{~W}, \mathrm{~T}_{2}{ }^{*}$ and are highest for both ratios. The non-normalized COVs, however, allow us to directly compare our results with those of earlier studies. One should bear in mind that these studies in some cases used different MR imaging approaches (e.g. FLASH images with specific magnetization preparations) with the sources of bias and variability that come with it. Nevertheless, the observed inter- and intra-subject $\mathrm{R}_{1}$ COV values within our GM regions (5.53\% and $1.63 \%$ ) are comparable with the inter-site COV (6\%) observed earlier (Weiskopf et al., 2013) and the reproducibility (2.08\%) observed for dGM structures (Okubo et al., 2016), respectively. Higher inter-site COV was also observed for the $T_{1} w(15.2 \%)$ vs. $R_{1}(6 \%)$ parameter by Weiskopf et al. (2013). It should be noted that the $T_{1} w$ approach as presented here, is largely comparable to the quantitative $\mathrm{R}_{1}$ map in terms of acquisition strategy, i.e. they are based on the same data. Standard $T_{1}$ w (e.g. MPRAGE) images are much more likely to show 
effects from MRI acquisition characteristics (Bock et al., 2013; Lorio et al., 2016). In our case, the lower inter- and intra-subject COV for $\mathrm{R}_{1}$ might therefore be a consequence of the non-linear way that $T_{1}$ values are computed from the $T_{1} w$ values in the MP2RAGE approach. In particular, a certain range of $\mathrm{T}_{1}$ values is translated into a broader range of $T_{1} w$ values, as illustrated in Figure 3 of the original paper by Marques et al. (2010), leading to a higher variation and lower reproducibility for the $T_{1} w$ maps compared to the $R_{1}$ maps. This, together with the fact that $R_{1}$ changes with age (Draganski et al., 2011; Grydeland et al., 2013; Callaghan et al., 2014) may explain the smaller difference observed between the $R_{1}$ and $T_{1}$ w intra-subject COV maps compared to the inter-subject COV maps, considering the age range of our study population (24 to 42 years). The highest COV for both ratio images could be explained by the fact that noise from both $\mathrm{R}_{1}$ (or $\mathrm{T}_{1} \mathrm{w}$ ) and $\mathrm{T}_{2}{ }^{*}$ measures propagate into the corresponding ratio images, leading to increased variability among subjects. Noise may be especially present in the $T_{2}{ }^{*}$ maps, as noise enhancement due to intensity fluctuations (i.e. increased SD) across echoes when fitting the GRE data possibly caused the higher (non-normalized) COV compared to the $\mathrm{T}_{2}{ }^{*} \mathrm{~W}$ maps and previous reported values based on data acquired similarly (Govindarajan et al., 2015). However, Govindarajan et al. (2015) used a 2D GRE sequence with anisotropic voxels and a high in-plane resolution $(0.3 \times 0.3 \times 1 \mathrm{~mm})$, therefore allowing a longer TR (2020 ms) to acquire more TEs ( $N=12)$, while boosting the SNR. Acquisition of GRE data using an increased number of echo times to enhance the $\mathrm{T}_{2}{ }^{*}$ fitting procedure significantly improves the COV. However, this also considerably increases the scanning time, which we kept as short and similar to the MP2RAGE as possible. For instance, in the Govindarajan et al. (2015) study covering only part of the brain, the GRE acquisition time was more than double of ours.

Note that for both quantitative $R_{1}$ (or $T_{1} w$ ) and $T_{2}{ }^{*}$, the spatial inter-subject COV maps are ideally not influenced by RF transmit field inhomogeneities. In case of $R_{1}$ (and $\mathrm{T}_{1} \mathrm{w}$ ) measures, possible deviations in the $\mathrm{B}_{1}^{+}$across subjects are removed by post-hoc correcting the MP2RAGE data using the acquired $\mathrm{B}_{1}{ }^{+}$-map (Eggenschwiler et al., 2012; Marques and Gruetter, 2013). For the ME-GRE data, the $\mathrm{B}_{1}{ }^{+}$-inhomogeneities are removed from the quantitative $\mathrm{T}_{2}{ }^{*}$ image by the virtue of the fitting process that separates all non- $\mathrm{T}_{2}{ }^{*}$ contributions into a separate component. The division of ME-GRE images acquired at different echo times and the fact that the same flip angle was used for all readouts ensures that the resulting $\mathrm{T}_{2}{ }^{*} \mathrm{~W}$ image is also free from RF bias fields. Nevertheless, for the $\mathrm{T}_{2}{ }^{*}(\mathrm{w})$ COV maps, higher values are especially observed in more confined regions that are potentially affected by an increased density of veins (e.g. V1/2) or large arteries (e.g. RSC and AC). As a result, voxels in these regions may be influenced by strong artifacts, such as susceptibility variations and increased partial 


\section{CHAPTER 3}

voluming, leading to significantly larger variations in the ME-GRE data within and across subjects close to the GM/CSF border. Also, the well-known $\mathrm{B}_{0}$-orientation dependence of the $\mathrm{T}_{2}{ }^{*}$ signal could have induced a slight decrease in the reproducibility of the $T_{2}^{*}(w)$ maps (Cohen-Adad et al., 2012).

\subsection{2. | Parcellability variation}

The FWHM of the distribution is a marker for the sensitivity of an MR parameter to differences in cortical values, on its own, if the image is not dominated by noise. However, in order to estimate how reliably a vertex value can be located within the cortical histograms and therefore how reliably it might be parcellated, a measure of noise should be taken into account. For example, the average intra-subject standard deviation for that specific vertex can serve as an estimate for non-biochemical variability in the image contrast. In the current study, a lower PV (i.e. a small standard deviation for a large FWHM) indicates a higher reliability to isolate that vertex's value. The longitudinal relaxation constants $\left(R_{1}\right.$ and $\left.T_{1} w\right)$ perform similarly better than the transversal relaxation constants $\left(\mathrm{T}_{2}{ }^{*}\right.$ and $\left.\mathrm{T}_{2}{ }^{*} \mathrm{~W}\right)$. The observed difference is predominantly induced by the lower reproducibility of the $\mathrm{T}_{2}{ }^{*}$ and $\mathrm{T}_{2}{ }^{*} \mathrm{~W}$ maps, since an advantageous (rescaled) FWHM was observed for the cortical $\mathrm{T}_{2}{ }^{*}$ distribution. This interpretation can also be extended to both ratio images. Taken together, the present data suggests that $\mathrm{R}_{1}$ and $\mathrm{T}_{1} \mathrm{w}$ maps are more reliable in parcellating the cortex.

\subsection{3. | Contrast-to-noise ratio}

For the definition employed in the current paper, CNR provides an additional indication of the sensitivity of each parameter to differentiate between heavily- and lightly-myelinated ROIs based on their average value and noise. Highest CNRs were observed for heavily-myelinated regions (e.g. BA1, visual cortex and auditory cortex) and lowest for lightly-myelinated regions (e.g. BA44, BA45 and RSC). Although, different MP2RAGE parameters (e.g. TR, $\mathrm{TI}_{1}$ and flip angles) were used than previously proposed for optimal CNR (Marques and Gruetter, 2013) to decrease the scan duration, $R_{1}$ and $T_{1} w$ maps were characterized by the highest CNR across most regions. Lowest CNRs were observed for both the $\mathrm{T}_{2}{ }^{*}$ and $\mathrm{T}_{2}{ }^{*} \mathrm{~W}$ parameters, which are not solely explained by differences in inter-subject variability, considering the fact that the highest variation was observed for both ratio images. However, this difference in CNR between the longitudinal $\left(R_{1}\right.$ and $\left.T_{1} w\right)$ and transversal $\left(T_{2}^{*}(w)\right)$ parameters may be caused by differences in their biochemical basis, with myelin and iron being the dominant contributors to the cortical differences in $R_{1}$ and $T_{2}{ }^{*}$, respectively (Stuber et al., 2014). This may also explain the smaller regional differences between $R_{1}$ (or $T_{1} w$ ) and $T_{2}{ }^{*}(w)$ CNR in the 
lightly-myelinated regions. In the RSC, lower CNR is observed for $R_{1}$ and $T_{1} w$, which is in agreement with the fact that previous studies reported highest myelin content in its layers close to the pial surface, which was excluded in our surface analysis, and lowest myelin content in its middle layers (Fatterpekar et al., 2002). However, since the CNR involves the absolute differences between two regions, artifacts induced by a large vein (vena magna cerebri) lead to significantly lower $\mathrm{T}_{2}{ }^{*}$ values in the RSC, and may account for an artificial enhancement of CNR for the $\mathrm{T}_{2}{ }^{*}(\mathrm{~W})$ maps in this region.

\subsection{4. | Whole-cortex myelin-related pattern}

The current data demonstrates good agreement in terms of the observed general myelin-related pattern across all investigated MR parameters and with earlier studies. As in previous studies, the primary areas, including M1, S1, V1 and AC, differed in their $\mathrm{T}_{1}$ - or $\mathrm{T}_{2}{ }^{*}$-values from the average values throughout the cortex. Noteworthy is the shift more posteriorly (i.e. corresponding to the gyral wall of Heschl's gyrus) that we observed with respect to the localization of the auditory core (here defined as the part of $A C$ with the highest degree of myelination) in the $\mathrm{T}_{2}{ }^{*}$ compared to $\mathrm{T}_{1}$ parameter. This difference was present in both the single-subject and group data and can therefore not be explained solely by misalignment due to the averaging. $\mathrm{B}_{0}$-orientation dependence of $\mathrm{T}_{2}{ }^{*}$ signal (Cohen-Adad et al., 2012) may have induced the slight difference in locations, but the precise effects of $B_{0}$-orientation remain to be established across the entire cortex. $R_{1}$ (or $T_{1} w$ ) and $T_{2}{ }^{*}(w)$ are especially (negatively) correlated in the deeper layers, which are generally characterized by the highest degree of myelination (Ding et al., 2016). Differences in the myelination across layers between the gyral wall and gyral crown of the Heschl's gyrus may possibly explain the observed difference in location of the auditory core between $\mathrm{R}_{1}$ and $\mathrm{T}_{2}{ }^{*}$.

The presented maps can be used to parcellate the whole brain into distinct regions based on strong changes in myeloarchitecture across the cortex (Geyer et al., 2011; Glasser and Van Essen, 2011; Glasser et al., 2016). However, since MR parameters values are by nature continuous, defining areal borders between regions with a distinct myeloarchitecture (i.e. characterized by a strong change in an MR value) requires an additional criterion to make the continuous value distribution to discontinuous border delineation. Two methods to define transitions between cortical regions were explored based on either (i) the contours that track vertices characterized by specific values (thresholds) or (ii) borders that track the peak locations of strong surface-based local gradients. Whereas the latter approach is argued to be observer-independent (Glasser et al., 2016), the first approach allows comparative analyses of the surface patterns between parameters as it provides continuous contours, but may also be 


\section{CHAPTER 3}

more affected by the chosen threshold. Although the observer-dependence of the proposed threshold approach was minimized as much as possible, confounding factors like local artifacts, outliers or global differences in the shape of the histograms may lead to misleading conclusions. Nevertheless, our analysis showed that any choice of value between the $60^{\text {th }}$ and $90^{\text {th }}$ percentage of the AUC will not dramatically influence the final conclusions of surface pattern analyses as done in this paper and may therefore be adjusted according to the characteristics of the ROI. Interestingly, the differences between parameters (e.g. $\mathrm{R}_{1}$ vs. $\mathrm{T}_{2}^{*}$ ) in the surface area delineated by these thresholds, were not uniform across regions. This may possibly be induced by differences in their biochemical composition (e.g. higher tissue iron content) or due to a higher density of blood vessels (leading to lower $T_{2}{ }^{*}(w)$ values) between the visual areas and somatosensory cortex, for example, respectively.

Alternatively, areal borders defined by the peak locations of myelin content change across the surface may provide additional supporting and more fine-scale information, such as the border between BA3 and BA4 or of the auditory core. However, as we demonstrate in the current work, the gradient method itself suffers from shortcomings and does not provide a completely objective approach for border definitions. The observed borders vary in quality depending on the parameter and are not continuous, therefore demanding observer input when comparing parameters (i.e. a choice of the gradient threshold value and filling-in gaps in the borders). This approach seems to be especially appropriate in group studies after including a significantly higher amount of subjects as demonstrated by Glasser and Van Essen (2011) and Glasser et al. (2016). Moreover, subsequent inter-subject surface registration methods that use multiple modalities (e.g. $T_{1}$, thickness and curvature) will additionally improve the quality of the gradients (Robinson et al., 2014; Tardif et al., 2015). Studies that want to localize and/or characterize the structural extent of specific regions within single-subjects (e.g. to restrain their functional analyses) would, however, not substantially benefit from this method as compared to the threshold method based on the current data. Within a single subject, more continuous gradients can be obtained by applying a higher smoothing factor across the surface, however, at the expense of sacrificing structural detail. In summary, given the continuous distribution of MRI values, both the threshold and the gradient methods require user input to define areal borders, and more improved algorithms and comparison with histology are necessary for objective, observer-independent cortical parcellation at the single-subject level. 


\subsection{5. | Technical considerations}

Despite the improvements in post-processing software tools and pipelines, accurate delineation of the gray and white matter borders is still difficult and manual correction of segmentation errors may have to be performed. Automatic segmentation of MP2RAGE data acquired at 7T is especially challenging in the lower parts of the brain that suffer from a low $\mathrm{B}_{1}{ }^{+}$-field and susceptibility effects. In the current study, these effects were partially eliminated by using dielectric pads (O'Brien et al., 2014) and by applying corrections for $\mathrm{B}_{1}^{+}$inhomogeneities (Eggenschwiler et al., 2012). Erroneous segmentation may lead to imperfect mapping of the parameters in these areas (e.g. at variable cortical depths) and misinterpretation of the data (Lutti et al., 2014; Waehnert et al., 2015).

\subsection{6. | Summary}

Our data acquired using a time-efficient imaging protocol (< $20 \mathrm{~min}$ ) strengthens the robustness of myelin-related cortical mapping across different (i) magnetic field strengths, (ii) MR parameters and (iii) sequences. Despite the similarities that were observed between $\mathrm{R}_{1}$ (or $\mathrm{T}_{1} \mathrm{w}$ ) and $\mathrm{T}_{2}{ }^{*}$ quantitative and weighted MRI with respect to myelination patterns and CNR per unit time, most importantly and optimally, MR parameters should be characterized by a high intra-subject reproducibility with minimal contamination by non-biochemical factors (e.g. MRI acquisition-related variations) and high reliability for cortical parcellation (i.e. low parcellability variation). Here, $R_{1}$ was characterized by (i) a low COV both within- and between-subjects and (ii) low parcellability variation, making it more suitable as a myelin-related MR biomarker compared to the other tested MR parameters for studies in learning, gender, age and diseases effects on the brain's microstructure. However, the most important argument to take into account when choosing sequences is that quantitative imaging (such as $R_{1^{\prime}}$ $\mathrm{T}_{2}{ }^{*}$ or their ratio) allows detecting absolute differences within and between subjects. Detecting heavily- and lightly-myelinated areas using weighted imaging relies on the fact that these areas differ in their MRI intensity from the average values in the brain. That is, the normalization of myelination distribution based on weighted-MRI intensity is typically done within a subject. However, it is conceivable that subjects differ not only in their relative distribution, but also in their absolute myelination, for example, due to disease and, in these cases, quantitative MRI parameters are most likely more suitable for inter-subject comparison. 


\section{CHAPTER 3}

\section{5. | REFERENCES}

Bock, N. A., E. Hashim, R. Janik, N. B. Konyer, M. Weiss, G. J. Stanisz, R. Turner and S. Geyer (2013). "Optimizing T1-weighted imaging of cortical myelin content at 3.0 T." Neuroimage 65: 1-12.

Bock, N. A., A. Kocharyan, J.V. Liu and A. C. Silva (2009). “Visualizing the entire cortical myelination pattern in marmosets with magnetic resonance imaging." J Neurosci Methods 185(1): 15-22.

Brodmann, K. (1909). Vergleichende Lokalisationslehre der Grosshirnrinde in ihren Prinzipien dargestellt auf Grund des Zellenbaues, Barth.

Callaghan, M. F., P. Freund, B. Draganski, E. Anderson, M. Cappelletti, R. Chowdhury, J. Diedrichsen, T. H. Fitzgerald, P. Smittenaar, G. Helms, A. Lutti and N. Weiskopf (2014). “Widespread age-related differences in the human brain microstructure revealed by quantitative magnetic resonance imaging." Neurobiol Aging 35(8): 1862-1872.

Callaghan, M. F., G. Helms, A. Lutti, S. Mohammadi and N. Weiskopf (2015). “A general linear relaxometry model of R1 using imaging data." Magn Reson Med 73(3): 1309-1314.

Cohen-Adad, J., J. R. Polimeni, K. G. Helmer, T. Benner, J. A. McNab, L. L. Wald, B. R. Rosen and C. Mainero (2012). "T(2)* mapping and $\mathrm{B}(0)$ orientation-dependence at $7 \mathrm{~T}$ reveal cyto- and myeloarchitecture organization of the human cortex." Neuroimage 60(2): 1006-1014.

De Martino, F., M. Moerel, J. Xu, P. F. van de Moortele, K. Ugurbil, R. Goebel, E. Yacoub and E. Formisano (2014). "High-Resolution Mapping of Myeloarchitecture In Vivo: Localization of Auditory Areas in the Human Brain." Cereb Cortex.

Deistung, A., A. Schafer, F. Schweser, U. Biedermann, D. Gullmar, R. Trampel, R. Turner and J. R. Reichenbach (2013). "High-Resolution MR Imaging of the Human Brainstem In vivo at 7 Tesla." Front Hum Neurosci 7: 710.

Dick, F., A. T. Tierney, A. Lutti, O. Josephs, M. I. Sereno and N. Weiskopf (2012). "In vivo functional and myeloarchitectonic mapping of human primary auditory areas." J Neurosci 32(46): 1609516105.

Ding, S. L., J. J. Royall, S. M. Sunkin, L. Ng, B. A. Facer, P. Lesnar, A. Guillozet-Bongaarts, B. McMurray, A. Szafer, T. A. Dolbeare, A. Stevens, L. Tirrell, T. Benner, S. Caldejon, R. A. Dalley, N. Dee, C. Lau, J. Nyhus, M. Reding, Z. L. Riley, D. Sandman, E. Shen, A. van der Kouwe, A. Varjabedian, M. Write, L. Zollei, C. Dang, J. A. Knowles, C. Koch, J. W. Phillips, N. Sestan, P. Wohnoutka, H. R. Zielke, J. G. Hohmann, A. R. Jones, A. Bernard, M. J. Hawrylycz, P. R. Hof, B. Fischl and E. S. Lein (2016). “Comprehensive cellular-resolution atlas of the adult human brain." J Comp Neurol.

Draganski, B., J. Ashburner, C. Hutton, F. Kherif, R. S. Frackowiak, G. Helms and N. Weiskopf (2011). "Regional specificity of MRI contrast parameter changes in normal ageing revealed by voxel-based quantification (VBQ)." Neuroimage 55(4): 1423-1434.

Droby, A., C. Lukas, A. Schanzer, I. Spiwoks-Becker, A. Giorgio, R. Gold, N. De Stefano, H. Kugel, M. Deppe, H. Wiendl, S. G. Meuth, T. Acker, F. Zipp and R. Deichmann (2015). "A human post-mortem brain model for the standardization of multi-centre MRI studies." Neuroimage 110C: 11-21.

Eggenschwiler, F., T. Kober, A. W. Magill, R. Gruetter and J. P. Marques (2012). "SA2RAGE: a new sequence for fast B1+-mapping." Magn Reson Med 67(6): 1609-1619.

Eickhoff, S., N. B. Walters, A. Schleicher, J. Kril, G. F. Egan, K. Zilles, J. D. Watson and K. Amunts (2005). "High-resolution MRI reflects myeloarchitecture and cytoarchitecture of human cerebral cortex." Hum Brain Mapp 24(3): 206-215.

Fatterpekar, G. M., T. P. Naidich, B. N. Delman, J. G. Aguinaldo, S. H. Gultekin, C. C. Sherwood, P. R. Hof, B. P. Drayer and Z. A. Fayad (2002). "Cytoarchitecture of the human cerebral cortex: MR microscopy of excised specimens at 9.4 Tesla." AJNR Am J Neuroradiol 23(8): 1313-1321.

Focke, N. K., G. Helms, S. Kaspar, C. Diederich, V. Toth, P. Dechent, A. Mohr and W. Paulus (2011). "Multi-site voxel-based morphometry--not quite there yet." Neuroimage 56(3): 1164-1170.

Freund, P., N. Weiskopf, J. Ashburner, K. Wolf, R. Sutter, D. R. Altmann, K. Friston, A. Thompson and A. Curt (2013). "MRI investigation of the sensorimotor cortex and the corticospinal tract after 
acute spinal cord injury: a prospective longitudinal study." Lancet Neurol 12(9): 873-881.

Fukunaga, M., T. Q. Li, P. van Gelderen, J. A. de Zwart, K. Shmueli, B. Yao, J. Lee, D. Maric, M. A. Aronova, G. Zhang, R. D. Leapman, J. F. Schenck, H. Merkle and J. H. Duyn (2010). “Layer-specific variation of iron content in cerebral cortex as a source of MRI contrast." Proc Natl Acad Sci U S A 107(8): 3834-3839.

Geyer, S., M. Weiss, K. Reimann, G. Lohmann and R. Turner (2011). “Microstructural Parcellation of the Human Cerebral Cortex - From Brodmann's Post-Mortem Map to in vivo Mapping with High-Field Magnetic Resonance Imaging." Front Hum Neurosci 5: 19.

Glasser, M. F., T. S. Coalson, E. C. Robinson, C. D. Hacker, J. Harwell, E. Yacoub, K. Ugurbil, J. Andersson, C. F. Beckmann, M. Jenkinson, S. M. Smith and D. C. Van Essen (2016). "A multi-modal parcellation of human cerebral cortex." Nature.

Glasser, M. F., M. S. Goyal, T. M. Preuss, M. E. Raichle and D. C. Van Essen (2014). “Trends and properties of human cerebral cortex: correlations with cortical myelin content." Neuroimage 93 Pt 2: 165-175.

Glasser, M. F., S. N. Sotiropoulos, J. A. Wilson, T. S. Coalson, B. Fischl, J. L. Andersson, J. Xu, S. Jbabdi, M. Webster, J. R. Polimeni, D. C. Van Essen, M. Jenkinson and W. U.-M. H. Consortium (2013). “The minimal preprocessing pipelines for the Human Connectome Project." Neuroimage 80: 105-124.

Glasser, M. F. and D. C. Van Essen (2011). “Mapping human cortical areas in vivo based on myelin content as revealed by T1- and T2-weighted MRI."J Neurosci 31(32): 11597-11616.

Govindarajan, S. T., J. Cohen-Adad, M. P. Sormani, A. P. Fan, C. Louapre and C. Mainero (2015). "Reproducibility of T2 * mapping in the human cerebral cortex in vivo at 7 tesla MRI." J Magn Reson Imaging 42(2): 290-296.

Grydeland, H., K. B. Walhovd, C. K. Tamnes, L.T. Westlye and A. M. Fjell (2013). “Intracortical myelin links with performance variability across the human lifespan: results from T1- and T2-weighted MRI myelin mapping and diffusion tensor imaging." J Neurosci 33(47): 18618-18630.

Koenig, S. H. (1991). "Cholesterol of myelin is the determinant of gray-white contrast in MRI of brain." Magn Reson Med 20(2): 285-291.

Langkammer, C., N. Krebs, W. Goessler, E. Scheurer, F. Ebner, K. Yen, F. Fazekas and S. Ropele (2010). "Quantitative MR imaging of brain iron: a postmortem validation study." Radiology 257(2): 455-462.

Lorio, S., F. Kherif, A. Ruef, L. Melie-Garcia, R. Frackowiak, J. Ashburner, G. Helms, A. Lutti and B. Draganski (2016). "Neurobiological origin of spurious brain morphological changes: A quantitative MRI study." Hum Brain Mapp 37(5): 1801-1815.

Lutti, A., F. Dick, M. I. Sereno and N. Weiskopf (2014). “Using high-resolution quantitative mapping of R1 as an index of cortical myelination." Neuroimage 93 Pt 2: 176-188.

Marques, J. P. and R. Gruetter (2013). “New developments and applications of the MP2RAGE sequence--focusing the contrast and high spatial resolution R1 mapping." PLoS One 8(7): e69294.

Marques, J. P., T. Kober, G. Krueger, W. van der Zwaag, P. F. Van de Moortele and R. Gruetter (2010). "MP2RAGE, a self bias-field corrected sequence for improved segmentation and T1-mapping at high field." Neuroimage 49(2): 1271-1281.

Miot-Noirault, E., L. Barantin, S. Akoka and A. Le Pape (1997). "T2 relaxation time as a marker of brain myelination: experimental MR study in two neonatal animal models." J Neurosci Methods 72(1): 5-14.

Nieuwenhuys, R., C. A. Broere and L. Cerliani (2014). "A new myeloarchitectonic map of the human neocortex based on data from the Vogt-Vogt school." Brain Struct Funct.

O'Brien, K. R., A. W. Magill, J. Delacoste, J. P. Marques, T. Kober, H. P. Fautz, F. Lazeyras and G. Krueger (2014). "Dielectric pads and low- B1+ adiabatic pulses: complementary techniques to optimize structural T1 w whole-brain MP2RAGE scans at 7 tesla." J Magn Reson Imaging 40(4): 804-812.

Okubo, G., T. Okada, A. Yamamoto, M. Kanagaki, Y. Fushimi, T. Okada, K. Murata and K. Togashi 


\section{CHAPTER 3}

(2016). "MP2RAGE for deep gray matter measurement of the brain: A comparative study with MPRAGE."J Magn Reson Imaging 43(1): 55-62.

Robinson, E. C., S. Jbabdi, M. F. Glasser, J. Andersson, G. C. Burgess, M. P. Harms, S. M. Smith, D. C. Van Essen and M. Jenkinson (2014). "MSM: a new flexible framework for Multimodal Surface Matching." Neuroimage 100: 414-426.

Rooney, W. D., G. Johnson, X. Li, E. R. Cohen, S. G. Kim, K. Ugurbil and C. S. Springer, Jr. (2007). "Magnetic field and tissue dependencies of human brain longitudinal $1 \mathrm{H} 2 \mathrm{O}$ relaxation in vivo." Magn Reson Med 57(2): 308-318.

Schmierer, K., F. Scaravilli, D. R. Altmann, G. J. Barker and D. H. Miller (2004). “Magnetization transfer ratio and myelin in postmortem multiple sclerosis brain." Ann Neurol 56(3): 407-415.

Sereno, M. I., A. Lutti, N. Weiskopf and F. Dick (2013). "Mapping the human cortical surface by combining quantitative T(1) with retinotopy." Cereb Cortex 23(9): 2261-2268.

Sigalovsky, I. S., B. Fischl and J. R. Melcher (2006). "Mapping an intrinsic MR property of gray matter in auditory cortex of living humans: a possible marker for primary cortex and hemispheric differences." Neuroimage 32(4): 1524-1537.

Stuber, C., M. Morawski, A. Schafer, C. Labadie, M. Wahnert, C. Leuze, M. Streicher, N. Barapatre, K. Reimann, S. Geyer, D. Spemann and R. Turner (2014). "Myelin and iron concentration in the human brain: a quantitative study of MRI contrast." Neuroimage 93 Pt 1: 95-106.

Tardif, C. L., A. Schafer, M. Waehnert, J. Dinse, R. Turner and P. L. Bazin (2015). “Multi-contrast multi-scale surface registration for improved alignment of cortical areas." Neuroimage.

Teeuwisse, W. M., W. M. Brink, K. N. Haines and A. G. Webb (2012). "Simulations of high permittivity materials for $7 \mathrm{~T}$ neuroimaging and evaluation of a new barium titanate-based dielectric." Magn Reson Med 67(4): 912-918.

Vogt, C. and O. Vogt (1919). Allgemeine ergebnisse unserer hirnforschung, JA Barth.

Waehnert, M. D., J. Dinse, A. Schafer, S. Geyer, P. L. Bazin, R. Turner and C. L. Tardif (2015). “A subject-specific framework for in vivo myeloarchitectonic analysis using high resolution quantitative MRI." Neuroimage.

Weiskopf, N., J. Suckling, G. Williams, M. M. Correia, B. Inkster, R. Tait, C. Ooi, E. T. Bullmore and A. Lutti (2013). "Quantitative multi-parameter mapping of R1, PD(*), MT, and R2 $\left(^{*}\right)$ at 3T: a multi-center validation." Front Neurosci 7: 95.

Zilles, K. and K. Amunts (2009). "Receptor mapping: architecture of the human cerebral cortex." Curr Opin Neurol 22(4): 331-339. 


\section{6. | SUPPLEMENTARY MATERIAL}

\subsection{1. | Subjectivity analysis}

Materials \& methods

For each inter- (e.g. $\mathrm{R}_{1}$ vs. $\mathrm{T}_{2}{ }^{*}$ ) and intra- (e.g. $\mathrm{R}_{1}$ vs $\mathrm{T}_{1}$ w) comparison, threshold and ROI, we (i) quantified the number of vertices and consecutively the total surface area $\left(\mathrm{mm}^{2}\right)$ using MATLAB; (ii) obtained the total area covered by the union of both parameters and (iii) retrieved the overlap percentage between parameters based on the number of vertices within each corresponding outline. This metric is also known as the Jaccard similarity coefficient $(J)$ and can be expressed using:

$$
J_{\mathrm{y}, T_{\mathrm{A}, \mathrm{B}}}(A, B)=\frac{\left|A_{\mathrm{y}, \mathrm{T}_{\mathrm{A}}} \cap B_{\mathrm{y}, \mathrm{T}_{\mathrm{B}}}\right|}{\left|A_{\mathrm{y}, \mathrm{T}_{\mathrm{A}}} \cup B_{\mathrm{y}, \mathrm{T}_{\mathrm{B}}}\right|}
$$

Here, the total area for each ROI (y) where parameters A and B (defined using threshold $T_{A}$ or $T_{B^{\prime}}$ respectively) intersect was divided by the total area for the union of $A$ and $B$. Subsequently, the total surface area and percentage of spatial overlap were then averaged across the three different thresholds to reduce the effect of noise on the cortical distribution of each parameter (and subsequent interpretations).

Results

Total surface area $\left(\mathrm{cm}^{2}\right)$ for each of the regions and thresholds were subsequently plotted per parameter in Fig. S3.3B and the computed spatial overlaps (\%, averaged across thresholds) between these areas are shown in Fig. S3.3C. Overall, higher spatial overlaps were observed between the weighted and quantitative images of the same parameter (i.e. intracontrast comparison) compared to the overlap between images from different parameters (i.e. intercontrast comparison). 


\section{CHAPTER 3}

\subsubsection{Supplementary figures}
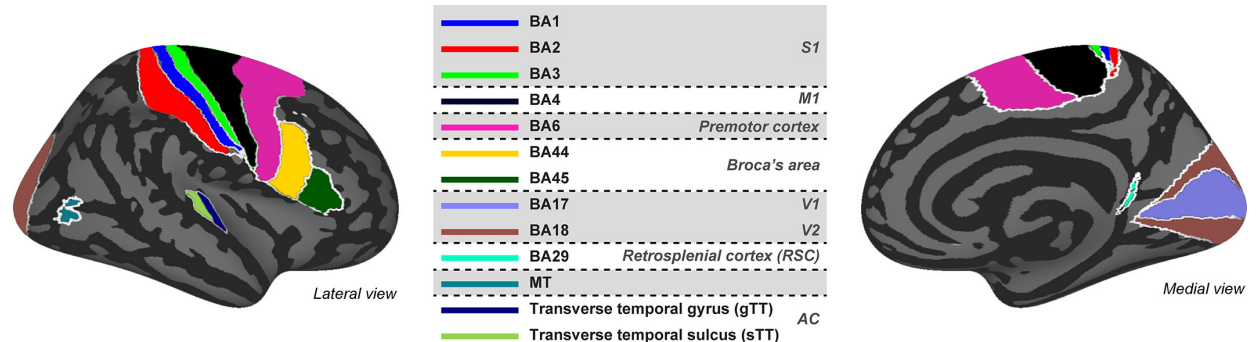

Fig. S3.1. | Overview of the different regions of interest (ROIs) used for analyses.
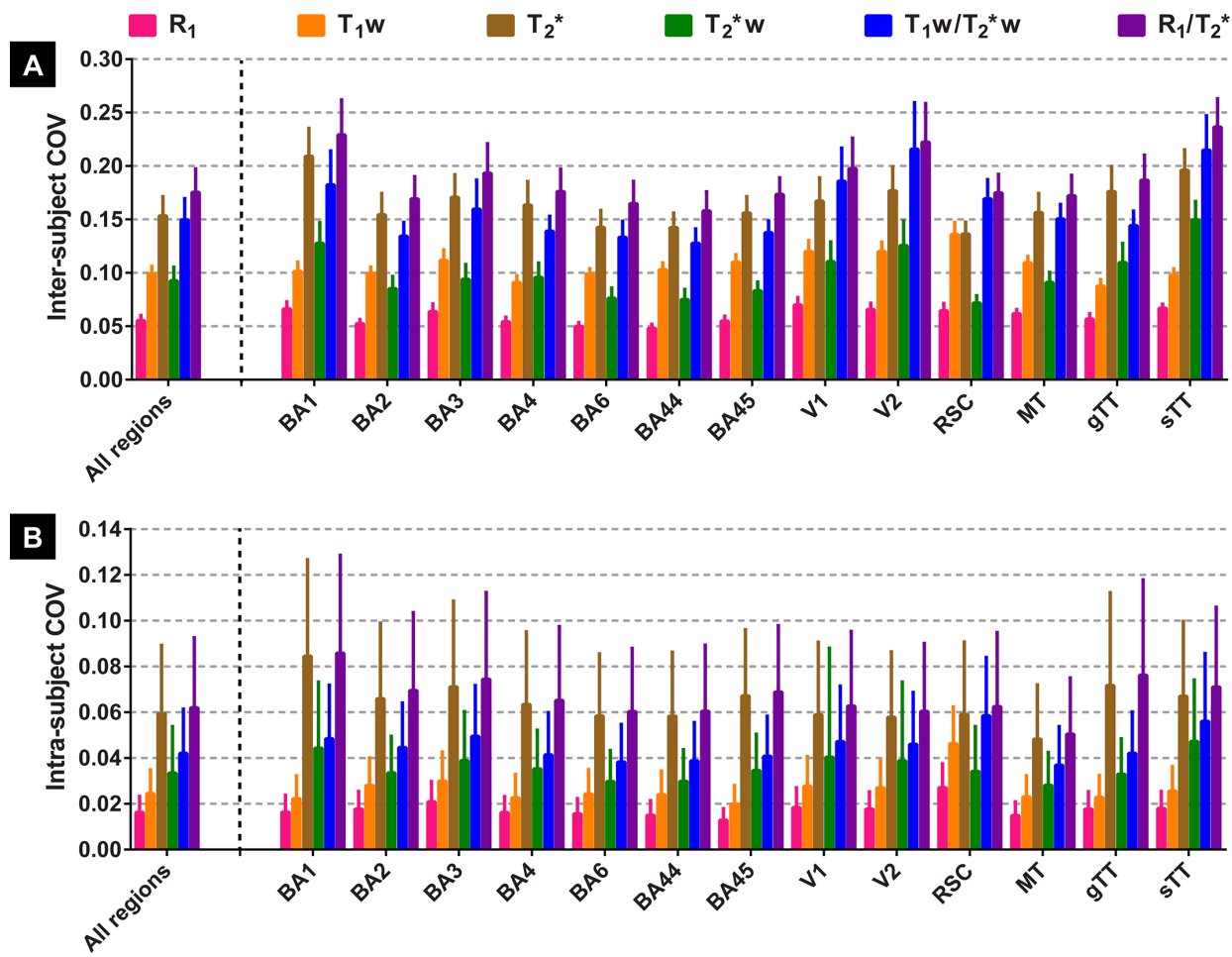

Fig. S3.2. | Average, non-normalized, inter- and intra-subject coefficients of variation (COV, A and B, respectively) comparison between the parameters investigated for the selected regions of interest (ROIs) and all ROls combined. 
Supplementary material

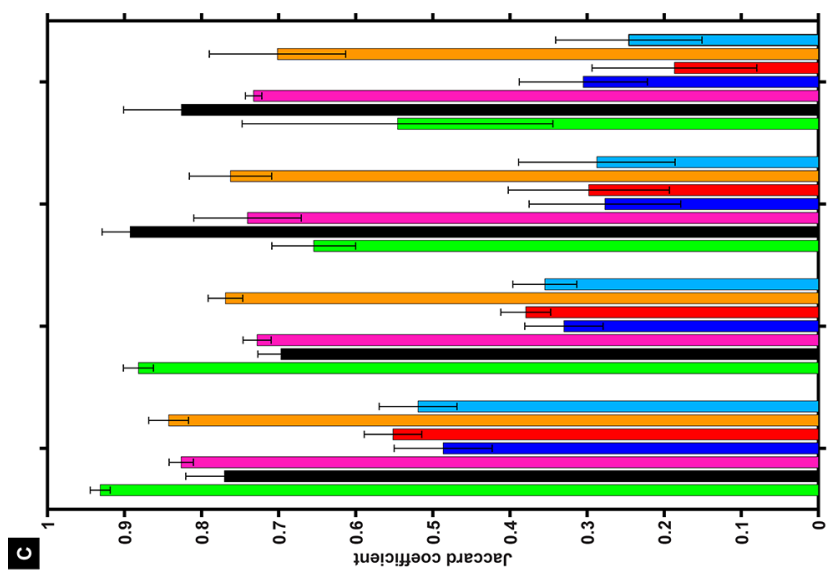

₹

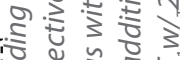
ปั่ 会齿:

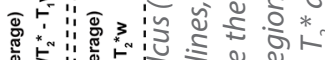
(1)

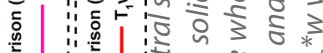

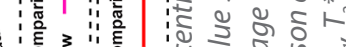

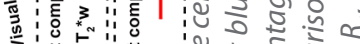
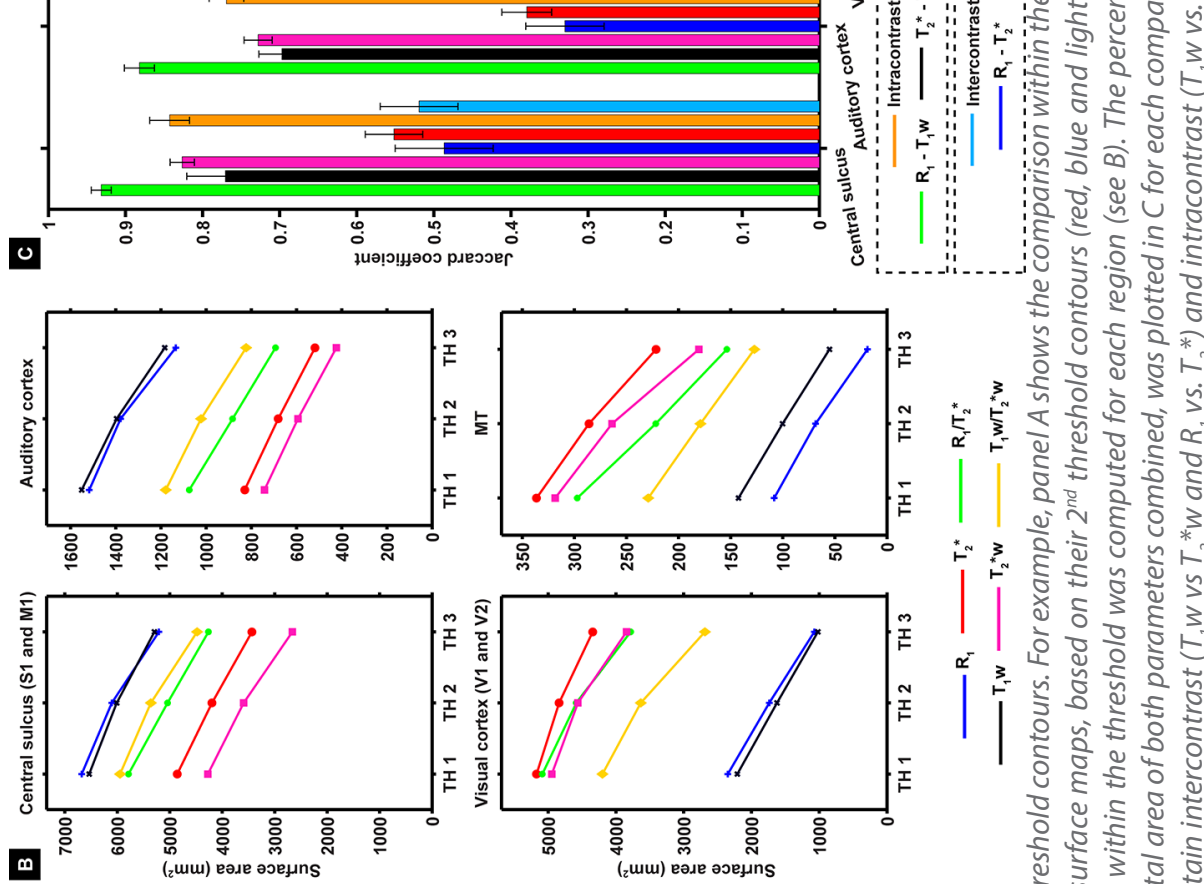
iิ ₹
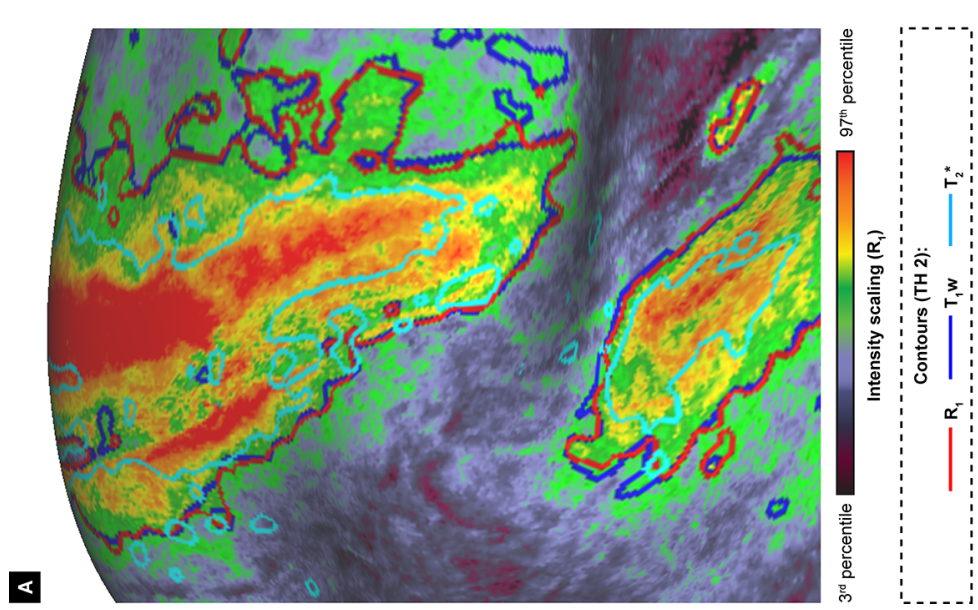
ร 둔 이워

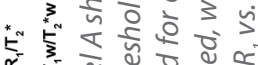
के

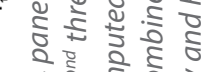

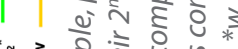

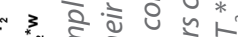

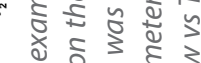
ฮ :

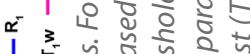

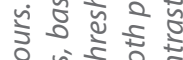

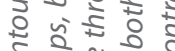
ठำ है

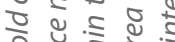

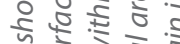

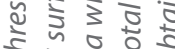

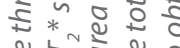

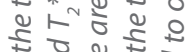

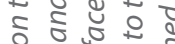
$23-5$ ติ 0 वा क्ष वे के क के

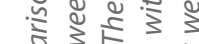

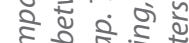
क्ष है है I 吾

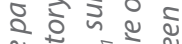

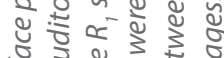

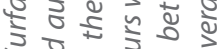

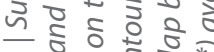
m. กิ

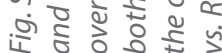




\section{CHAPTER 3}
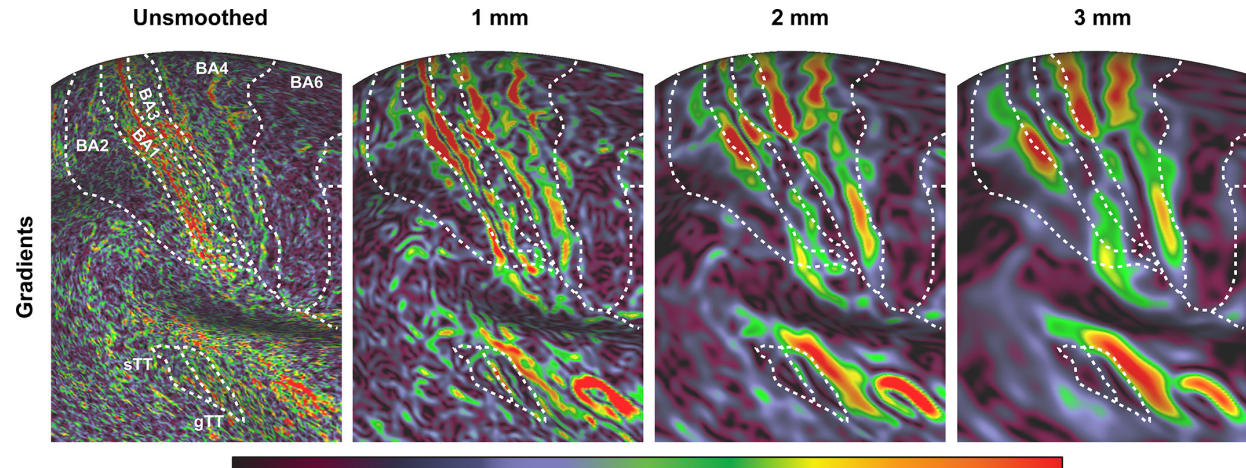

3

Intensity scaling (percentiles)

97

Fig. S3.4. | Surface pattern comparison based on gradient maps from the $R_{1}$ parameter after applying different smoothing levels. Local maximum gradients maps were computed based on the group average $R_{1}$ surface map (see Fig. 3.2) after applying different smoothing kernels: unsmoothed, $1 \mathrm{~mm}$, $2 \mathrm{~mm}$ or $3 \mathrm{~mm}$. No significant improvement above a kernel size of $1 \mathrm{~mm}$ can be observed. 


\title{
ANATOMIC \& METABOLIC BRAIN MARKERS OF THE M.3243A>G MUTATION: A MULTI-PARAMETRIC 7 TESLA MRI STUDY
}

\author{
ROY A.M. HAAST \\ DIMO IVANOV \\ RUTGER J.T. IJSSELSTEIN \\ SUZANNE C.E.H. SALLEVELT \\ JACOBUS F.A. JANSEN \\ HUBERT J. M. SMEETS \\ IRENAEUS F.M. DE COO \\ ELIA FORMISANO \\ \& KÂMIL ULUDAĞ
}

\section{Corresponding publication:}

"Anatomic \& Metabolic Brain Markers of the m.3243A> G Mutation: a Multi-Parametric 7 Tesla MRI Study”. Neurolmage: Clin. 18 (2018). 


\section{CHAPTER 4}

\section{ABSTRACT}

One of the most common mitochondrial DNA (mtDNA) mutations, the A to G transition at base pair 3243, has been linked to changes in the brain, in addition to commonly observed hearing problems, diabetes and myopathy. However, a detailed quantitative description of $m .3243 A>G$ patients' brains has not been provided so far. In this study, ultra-high field MRI at 7 Tesla and volume- and surface-based data analyses approaches were used to highlight morphology (i.e. atrophy)-, microstructure (i.e. myelin and iron concentration)- and metabolism (i.e. cerebral blood flow)-related differences between patients $(\mathrm{N}=22)$ and healthy controls $(\mathrm{N}=15)$. The use of quantitative MRI at 7 Tesla allowed us to detect subtle changes of biophysical processes in the brain with high accuracy and sensitivity, in addition to typically assessed lesions and atrophy. Furthermore, the effect of $m .3243 A>G$ mutation load in blood and urine epithelial cells on these MRI measures was assessed within the patient population and revealed that blood levels were most indicative of the brain's state and disease severity, based on MRI as well as on neuropsychological data. Morphometry MRI data showed a wide-spread reduction of cortical, subcortical and cerebellar gray matter volume, in addition to significantly enlarged ventricles. Moreover, surface-based analyses revealed brain area-specific changes in cortical thickness (e.g. of the auditory cortex), and in $\mathrm{T}_{1}, \mathrm{~T}_{2}{ }^{*}$ and cerebral blood flow as a function of mutation load, which can be linked to typically m.3243A>G-related clinical symptoms (e.g. hearing impairment). In addition, several regions linked to attentional control (e.g. middle frontal gyrus), the sensorimotor network (e.g. banks of central sulcus) and the default mode network (e.g. precuneus) were characterized by alterations in cortical thickness, $T_{1}, T_{2}{ }^{*}$ and/or cerebral blood flow, which has not been described in previous MRI studies. Finally, several hypotheses, based either on vascular, metabolic or astroglial implications of the m.3243A>G mutation, are discussed that potentially explain the underlying pathobiology. To conclude, this is the first 7T and also the largest MRI study on this patient population that provides macroscopic brain correlates of the m.3243A $>\mathrm{G}$ mutation indicating potential MRI biomarkers of mitochondrial diseases and might guide future (longitudinal) studies to extensively track neuropathological and clinical changes.

\section{Acknowledgment}

The authors are indebted to Prof. Dr. Andrew Webb (Leiden University Medical Centre, Leiden, Netherlands), Dr. José Marques (Donders Institute for Brain, Cognition and Behaviour, Nijmegen, Netherlands) and Dr. Florence van Tienen for providing the dielectric pads, the MATLAB code to perform the $T_{1}$ correction and help with the genetic analyses used in this study, respectively. This work was supported by Maastricht University, the Netherlands Organization for Scientific Research (NWO; VIDI grant 452-11-002 to K.U.), Technology Foundation STW (12724 to E.F.) and Ride4Kids, Join4Energy and NeMo (to I.F.M.d.C.). 


\section{1. | INTRODUCTION}

The brain contributes to about $20 \%$ of the human body's energy consumption (Sokoloff et al., 1977; Magistretti and Allaman, 2015). Mitochondria serve as the biological units that utilize oxygen and glucose through oxidative phosphorylation to produce ATP from ADP (adenosine diphosphate). Approximately 4.7 billion ATP molecules per second are used by a single cortical neuron in a resting human brain, illustrating the extremely high energy demand of the brain (Zhu et al., 2012). As a result, a reduced energy production due to mitochondrial dysfunctioning may affect neuronal and glial integrity, leading to changes in the brain's structure and functioning (Keogh and Chinnery, 2015). For example, using MRI, qualitative cortical, white matter, brainstem and cerebellar morphological changes were observed in patients with respiratory energy chain defects (Saneto et al., 2008). In addition, changes are observed related to the homeostasis of biochemical compounds (such as iron, myelin and calcium), as particularly highlighted by deep gray matter MRI signal intensity changes (Dinopoulos et al., 2005).

Mitochondrial dysfunctioning can be caused by mutations in the mtDNA. Germline mutations can either occur de novo or be inherited from the mother. These mutations can be either homoplasmic, i.e. all mtDNA copies mutated, or heteroplasmic, i.e. a mixture of mutated and wild-type mtDNA. For heteroplasmic mutations, the mutation load may differ among different tissue types. As mitochondria are present in most eukaryotic cell types, mitochondrial mutations lead to a wide-range of symptoms, affecting multiple systems in the body, especially those with a high energy requirement, including the central nervous system (Lightowlers et al., 2015).

One of the most common mtDNA mutations is the m.3243A $>\mathrm{G}$ mutation within the mtDNA-encoded tRNA leucine 1 (MTTL1) gene (Goto et al., 1990), affecting proper incorporation of the amino acid leucine in mtDNA encoded proteins. The m.3243A>G mutation causes a broad variety of clinical manifestations, of which the MELAS or MIDD syndromes are the most common (i.e. $10 \%$ and $38 \%$ of m.3243A $>$ G carriers, respectively). These patients are characterized by a wide range of phenotypic expressions, but most often suffer from sensorineural (due to dysfunctioning cochlear hair cells or nerve) hearing loss (51\%), diabetes (42\%) and proximal myopathy (27\%) (Nesbitt et al., 2013). m.3243A>G specific brain symptoms are (transient) SLEs, visible as $\mathrm{T}_{2}$ MRI signal hyperintensities and characterized by an increased $C B F$, as measured using ASL. Increased CBF is detected both before (Ikawa et al., 2013) and after onset of SLEs (Li et al., 2017), possibly to counterbalance observed neuronal hyperexcitability 


\section{CHAPTER 4}

and pathology (lizuka et al., 2002). Since SLEs are an important feature of the brain pathology in m.3243A>G patients, several studies have attempted to characterize the pathogenesis, as well as the temporal and spatial progression of these lesions. For example, these studies have shown locally increased lactate levels (Tsujikawa et al., 2010), but also a reduced apparent diffusion coefficient and cortical laminar necrosis (lizuka et al., 2003). In addition, increased baseline CBF was observed in m.3243A>G patients without SLEs (Rodan et al., 2015).

However, not all patients fall within the 'standard' disease criteria, leading to sub-optimal and delayed diagnosis and prognosis. Several attempts have been made to link m.3243A>G mutation load in different tissue types (e.g. skeletal muscle, blood leukocytes and urine) with disease severity (based on, for example, number of symptoms or severity scores) to predict clinical prognosis (Chinnery et al., 1997; Whittaker et al., 2009; de Laat et al., 2012). Mutation load in UECs was preferred, based on its (1) strong correlation with clinical parameters, (2) stability throughout life and (3) non-invasive assessment. Based on the above criteria, it is evident that the m.3243A>G mutation induces a large diversity of symptoms, including different types of brain changes. While the severity of the phenotype may depend on mutation load, no previous study focused on the mutation load-brain relationship in m.3243A $>$ G patients. Thus, it is not clear how the mutation load is associated with cortical, subcortical and cerebellar pathologies on microscopic and macroscopic levels and how those relate to the individual's clinical symptoms.

In the present neuroimaging study, we aim to quantify the relationship between the $\mathrm{m} .3243 \mathrm{~A}>\mathrm{G}$ mutation load percentage and brain phenotype in a large group of patients $(\mathrm{N}=22)$ and healthy controls $(\mathrm{N}=15)$ using UHF 7T MRI. State-of-the-art quantitative brain imaging and analysis approaches are utilized that enable a direct comparison between groups and within patients, with a focus on the brain's morphological (e.g. brain volume and cortical thickness), microstructural-related ( $T_{1}$ and $T_{2}{ }^{*}$ relaxometry) and metabolism-related (CBF) properties. More specifically, we investigate (1) the relationship between (sub-)cortical and cerebellar atrophy with the m.3243A $>$ G genotype (i.e. no mutation vs. low to high mutation load) and (2) the potential differences in myelin- and iron-sensitive $T_{1}, T_{2}^{*}$, as well as CBF, colocalizing with the morphological changes in patients.

The relative gain in SNR at 7T compared to clinical 1.5T and 3T depends on the MRI contrast used (Vaughan et al., 2001; Norris, 2003; Pohmann et al., 2016). For CBF using ASL the gain is approximately linear for high-resolution studies (e.g. below $2 \mathrm{~mm}$ 
isotropic). For example, in the study of Ivanov et al. (2017), 7T outperformed 3T by a factor of $\sim 1.75$ at $1.5 \mathrm{~mm}$ in-plane resolution for CBF data. For susceptibility contrasts, such as $\mathrm{T}_{2}^{*}$ and functional MRI, the gain can be more than linear. Uludag and Blinder (2017)have theoretically estimated that the SNR of fMRI at high spatial resolution scales approximately quadratically with magnetic field strength. For $\mathrm{T}_{1}$ contrast, however, a signal gain is only expected for very high resolution, higher than typically utilized in in vivo human studies.

Several studies already provided clear evidence of the enhanced detection of disease-specific morphological changes in the brains of, for example, multiple sclerosis and Alzheimer's disease patients using 7T compared to 3T (Nakada et al., 2008; Kollia et al., 2009). Studies, comparing lower to UHF-MRI for a wide range of clinical applications, have been discussed recently by Trattnig et al. (2016). Here, the improved SNR for $7 \mathrm{~T}$ enriches the images with spatial details not visible at lower field strengths due to the possibility to acquire data with a higher resolution and CNR, but without increasing the scan time (Duyn et al., 2007). As a result, 7T imaging exhibits increased diagnostic power due to enhanced sensitivity to detect fine-scale structural (Stuber et al., 2014; Keuken et al., 2017), perfusion (Gardener et al., 2009; Ivanov et al., 2017) and functional (Pfeuffer et al., 2002; Yacoub et al., 2008; Uludag and Blinder, 2017) properties and changes in the brain, see also recent reviews by van der Zwaag et al. (2016) and Ugurbil (2017). As such, the current results contribute to a better understanding of the disease pathology and classification of m.3243A>G patients, in particular, and mitochondrial disease patients, in general.

\section{2. | MATERIALS \& METHODS}

\subsection{1. | Participants}

Twenty-two m.3243A>G patients and fifteen healthy age-, gender- and education-matched controls were included in this study after providing written informed consent in accordance with the Declaration of Helsinki. The protocol was approved by the ethics review board of the MUMC+ in Maastricht, The Netherlands. We included patients only when they were ADL-independent, as measured using the Barthel disability index and had a score $<30$ on the NMDAS (Schaefer et al., 2006). We used three (current function, system specific involvement, and current clinical assessment) out of the four NMDAS sections, which resulted in a spectrum with less severe phenotypes to demonstrate the usefulness of quantitative MRI at 7T for detecting brain changes beyond lesions and atrophy, as it is typically assessed in imaging studies. For this reason, patients in the acute phase and/or with a history of SLEs were excluded. For severe 


\section{CHAPTER 4}

phenotypes (i.e. Barthel index $<15$ and/or NMDAS $>30$ ), the brains of $m .3243 A>G$ patients strongly deviate from those of age-matched controls, already detectable with standard MRI methods at 1.5 and 3T. Patients were only eligible for inclusion after evaluation of the clinically-relevant symptoms by an experienced clinician (I.F.M.d.C, see Fig. 4.1c for an overview of the symptoms). Patients with metallic implants (e.g. cochlear or stents) were excluded, as these would interfere with the magnetic field of the MRI scanner. Assessed parameters were: (1) m.3243A>G mutation loads (\%) in blood and UECs following the procedure described in (Sallevelt et al., 2013), (2) fasting blood glucose (mmol/L) and $\mathrm{HbA} 1 \mathrm{c}$ (\% and IFCC) levels measured using standard procedures and (3) cognitive performance scores. Several cognitive domains were assessed using the visual 15-WLT to test memory, recall and recognition (Van der Elst et al., 2005), the Stroop color-word test to test attention (Van der Elst et al., 2006) and the LDST to test information processing speed (van der Elst et al., 2006). Raw test scores were Z-scored based on the average control scores for each cognitive task (see Table 4.1).

\subsection{2. | Data acquisition}

MRI data were acquired using a whole-body 7T magnet (Siemens Medical Systems, Erlangen, Germany) and a 32-channel phased-array head coil (Nova Medical, Wilmington, MA, USA). High resolution ( $0.7 \mathrm{~mm}$ isotropic nominal voxel size) whole-brain quantitative $T_{1}$ and $T_{2}{ }^{*}$ images were obtained with MP2RAGE (Marques et al., 2010) and ME-GRE sequences, respectively. The SA2RAGE (Eggenschwiler et al., 2012) sequence was used to map $\mathrm{B}_{1}{ }^{+}$across the brain. In addition to the anatomical scans, baseline partial-brain (excluding cerebellum and lower temporal lobes) perfusion was quantified using FAIR QUIPSS II ASL (Ivanov et al., 2017). The eye centers were taken as reference for the magnet isocenter position to improve the ASL labeling efficiency. See Supplementary Table 4.1 for the relevant sequence parameters. In addition, a standard dual-echo field map was acquired to correct the ASL data for EPI readout-related geometrical distortions. Dielectric pads containing a $25 \%$ suspension of barium titanate in deuterated water were placed proximal to the temporal lobe areas to locally increase the transmit $\mathrm{B}_{1}^{+}$field and to improve its homogeneity across the brain (Teeuwisse et al., 2012).

Table. 4.1 | Acquisition parameters.

\begin{tabular}{l|l|l|l|l} 
& \multicolumn{1}{c}{ T $_{1}$} & \multicolumn{1}{c}{ T. $^{*}$} & \multicolumn{1}{c}{ CBF } \\
& \multicolumn{1}{c}{ MP2RAGE } & Sa2RAGE & ME-GRE & \multicolumn{1}{c}{ ASL QUIPSII } \\
\hline Voxel size $\left(\mathrm{mm}^{3}\right)$ & 0.7 & 2.0 & 0.7 & 2.8 \\
\hline TR $(\mathrm{ms})$ & 5000 & 2400 & 33 & 2500 \\
\hline
\end{tabular}




\begin{tabular}{l|l|l|l|l} 
& \multicolumn{1}{c}{ MP2RAGE } & \multicolumn{1}{c}{ Sa2RAGE } & \multicolumn{1}{c}{ ME-GRE } & \multicolumn{1}{c}{ ASL QUIPSII } \\
\hline $\mathrm{TI}_{\mathbf{1}} / \mathrm{TI}_{\mathbf{2}}$ (ms) & $900 / 2750$ & $58 / 1800$ & - & $700 / 1800$ \\
\hline $\mathrm{TE}(\mathbf{s})$ (ms) & 2.47 & 0.78 & $\begin{array}{l}2.53 / 7.03 / \\
12.55 / 20.35\end{array}$ & 11 \\
\hline Flip angle(s) () & $5 / 3$ & $4 / 11$ & 11 & 67 \\
\hline Partial fourier & $6 / 8$ & $6 / 8$ & $6 / 8$ & $6 / 8$ \\
\hline Phase-encoding & A-P & A-P & L-R & A-P \\
\hline GRAPPA & 3 & 2 & 2 & 2 \\
\hline Reference lines & 24 & 24 & 30 & 36 \\
\hline Numbe of slices & 240 & 88 & 208 & 24 \\
\hline Field of view (mm) & $224 \times 224$ & $256 \times 256$ & $224 \times 159$ & $196 \times 196$ \\
\hline Matrix (mm) & $224 \times 244 \times 244$ & $128 \times 128 \times 96$ & $320 \times 227 \times 208$ & $70 \times 70$ \\
\hline Acq. time (min) & $8: 02$ & $2: 16$ & $8: 33$ & $8: 02$ \\
\hline
\end{tabular}

\subsubsection{Data pre-processing pipeline}

The following pre-processing steps were applied. First, skull-stripping was performed by using the different MP2RAGE output volumes (UNI, T map and INV2; see Marques et al. (2010)). The INV2 image was used to obtain a brain mask, as it provides the best intra- and extra-cranial tissue contrast, especially after removal of any RF bias field using the Advanced Normalization Tools (ANTs)' N4BiasFieldRemoval tool (Tustison et al., 2010). Remaining non-brain tissue was removed using probability maps of the dura mater and arteries. These initial steps were performed using MIPAV 7.1.1 (Center for Information Technology, NIH, Bethesda, MD, USA), JIST 3.0 (Johns Hopkins University, Baltimore, MD, USA) and CBS High-Res Brain Processing tools 3.0.9 (Max Planck Institute for Human Cognitive and Brain Sciences, Leipzig, Germany). Both the MP2RAGE UNI and $\mathrm{T}_{1}$ images were post-hoc-corrected for variations in $\mathrm{B}_{1}{ }^{+}$using the same method as described in (Marques and Gruetter, 2013), and a $T_{2}{ }^{*}$ map was obtained from the GRE data using a mono-exponential fit $\left(f(T E)=S_{0} e^{-T E / T_{2} *}\right.$ ). Second, gradient non-linearity correction was applied on the brain extracted MP2RAGE UNI and other volumes using the gradient coefficients file provided by the scanner manufacturer and the Human Connectome (HCP) high-res analysis 'gradunwarp' tool (https://github.com/ Washington-University/gradunwarp). Finally, the skull-stripped and gradient distortion unwarped MP2RAGE UNI volume was aligned using affine transformation to the MNI space $(0.7 \mathrm{~mm})$ template.

All volumes within the ASL data and the $M_{0}$ volume were co-registered to the first volume to correct for subject motion during the run using FSL's MCFLIRT tool and 6 


\section{CHAPTER 4}

DOF (https://fsl.fmrib.ox.ac.uk). Each motion-corrected ASL run was then split into control and label time-series. The average of the label images was subtracted from the average of the control images to obtain a perfusion-weighted map. Control-label pairs affected by artifacts were excluded from the averaging procedure. Because $\mathrm{TI}_{2}$ slightly differed between the slices, the perfusion-weighted map was corrected using the actual slice-specific $\mathrm{TI}_{2}$ before the CBF calculation. Quantification of the perfusion map was performed according to the model described in Alsop et al. (2015):

$$
C B F=\frac{6000 \cdot \lambda \cdot \Delta M \cdot e^{\frac{T I_{2}}{T 1_{\text {blood }}}}}{2 \cdot \alpha \cdot T I_{1} \cdot M_{0}}
$$

assuming $T_{1, \text { blood }}=2.1 \mathrm{~s}$ (Dobre et al., 2007), $\lambda$ (brain/blood partition coefficient) $=0.9$ $\mathrm{ml} / \mathrm{g}$, a (labeling efficiency) $=0.95$. The factor 6000 converts the units from $1 / \mathrm{s}$ to $\mathrm{ml} / 100 \mathrm{~g} / \mathrm{min}$, which is the physiological unit for CBF.

\subsection{4. | Surface-based analyses}

Images were pre-processed to improve subsequent automatic volume- and surface-based processing. An extensive description of the pipeline can be found in the Supplementary Methods 1. The pre-processing included brain masking, post-hoc $T_{1}$ correction and computation of $\mathrm{T}_{2}{ }^{*}$ and ASL maps. Cortical reconstruction and submillimeter volumetric segmentation was then performed with the FreeSurfer (v6.0, http:// surfer.nmr.mgh.harvard.edu/) image analysis suite using the pre-processed MP2RAGE UNI images as input (Dale et al., 1999). Manual corrections of the tissue classifications were performed when necessary. Boundary-based registration (i.e. 'bbregister') was used to co-register the $\mathrm{T}_{2}{ }^{*}$ and CBF maps to the MP2RAGE data with a 6 DOF transformation and spline interpolation. In addition, the fieldmap was used to correct for EPI readout geometrical distortions and improve the co-registration of the CBF map, particularly near the sinuses. Co-registered CBF maps were then corrected for partial volume effects by dividing it with a GM probability map obtained using SPM12 (http:// www.fil.ion.ucl.ac.uk/spm). For each subject, all modalities were projected onto the surface using FreeSurfer's mri_vol2surf function by averaging between 20 and $80 \%$ of the cortical thickness (with steps of $0.05 \%$ ) to reduce potential partial voluming with WM and CSF. In addition, WM surface maps were computed by averaging between $-0.5 \mathrm{~mm}$ and $-2 \mathrm{~mm}$ distance (with steps of $0.05 \mathrm{~mm}$ ) from the WM-GM boundary. All surface maps, including surface-based morphology metrics generated by FreeSurfer (e.g. cortical volume and thickness), were coregistered to the 'fsaverage' subject using sphere-based alignment (Fischl et al., 1999) and smoothed with FWHM $=10 \mathrm{~mm}$ for further statistical analyses. Final surface maps were visualized using the Connectome Workbench v1.2.3 viewer (Washington University School of Medicine, Saint Louis, MO, 
USA) after conversion of the inflated surfaces and overlays to a compatible format. Non-cortical tissue in between the hemispheres was masked using FreeSurfer's parcellation scheme to avoid inappropriate scaling of the surface maps.

\subsection{5. | Volume-based analyses}

In addition to the surface-based data, volumetric data were assessed for subcortical structures and cerebellum. For the CN, GP and Pu, the automatic subcortical parcellation by FreeSurfer (Fischl et al., 2002) was manually corrected by taking into account the microstructural information (i.e. the values) from both the quantitative $T_{1}$ and $T_{2}{ }^{*}$ maps using ITK-SNAP v3.6.0 (Yushkevich et al., 2006). In addition, RN, SN and DN were semi-automatically delineated, navigated by a threshold-based approach implemented in ITK-SNAP. The cerebellar segmentation tool (CERES) was utilized to accurately segment the cerebellum into GM and WM (Romero et al., 2017). Final subcortical and cerebellar labels were used to export volume $\left(\mathrm{mm}^{3}\right)$ and average $T_{1}, T_{2}{ }^{*}$ and $C B F$ values for each of the structures to MATLAB and/or SPSS for further statistical analyses.

\subsection{6. | Statistical analyses}

Descriptive participants' characteristics are reported as mean \pm standard deviation (S.D.), while the median and IQR ( $25^{\text {th }}$ and $75^{\text {th }}$ percentiles) are shown for the NMDAS. Between-group (controls vs. m.3243A $>\mathrm{G}$ ) demographic characteristics were tested by use of independent 2-sample t-tests (continuous data) or Pearson $x^{2}$-tests (categorical data) with SPSS (Statistical Package for Social Sciences, version 23, IBM Corp., USA) and $a=0.05$.

For the volumetric data, a MANOVA test with Bonferroni correction was conducted to compare CGM, WM, subcortical GM (global or per structure) and cerebellar GM and WM volumes, $T_{1}, T_{2}{ }^{*}$ and CBF between groups. Each patient was assigned to one of two groups based on mutation load. However, due to differences in mutation load across tissue types (and, in blood, also with age, see for example de Laat et al. (2012)), it is difficult to predefine the pathogenic threshold level (Chomyn et al., 1992) for brain tissue based on the blood and/or UEC data. Therefore, the median was used as classifier within the entire patient population. This was based on pragmatic reasons (i.e. to have equal sub-group sizes with comparable statistical power) in order to perform group-wise analyses (besides correlation). In addition, linear regression analysis was used to explore the relationship between mutation load and the structures volumes, $\mathrm{T}_{1}, \mathrm{~T}_{2}{ }^{*}$ and $C B F$, and was therefore restricted to $\mathrm{m} .3243 \mathrm{~A}>\mathrm{G}$ patients. All tests were corrected for age, gender and eTIV. 


\section{CHAPTER 4}

For the cortical surface data, a two-step analysis approach was used, due to the high clinical variability between the m.3243A $>G$ patients, for detection of (1) within- and (2) between-group brain differences. First, a whole-brain vertex-wise linear regression analysis was performed to detect clusters of vertices, for which cortical thickness (as measure of cortical atrophy), $\mathrm{T}_{1}, \mathrm{~T}_{2}{ }^{*}$ and $\mathrm{CBF}$ are significantly negatively or positively correlated with mutation load (in blood) in m.3243A>G patients. Here, age and gender were used as nuisance variables and corrected for multiple comparisons (i.e. number of vertices in left and right hemispheres) using Monte Carlo Null-Z simulation with a voxel-wise threshold of 2.0 (cluster-wise $p$-value of $<0.01$ ). A more lenient cluster-wise $\mathrm{p}$-value of $<0.05$ was used for $\mathrm{T}_{1}, \mathrm{~T}_{2}{ }^{*}$ and $\mathrm{CBF}$ in a separate, additional analysis to also highlight subtle, but still significant, changes as a function of mutation load. This was motivated by the hypothesis that $T_{1}, T_{2}{ }^{*}$ and CBF differences do not per se have to concur with, but can also pre- and/or proceed cortical thickness alterations. Second, significant clusters (per parameter) were then used to compare between groups using an ANOVA test, corrected for age and gender.

\section{3. | RESULTS}

The healthy control subjects $(\mathrm{N}=15)$ were matched to the $\mathrm{m} .3243 \mathrm{~A}>\mathrm{G}$ patients $(\mathrm{N}=22)$ on the basis of age (Pearson $X^{2}$ test, $\left.p=0.487\right)$, gender $(p=0.538)$ and educational level $(p=0.838)$, see Table 4.2. Significant higher fasting glucose (ANOVA, $F_{1,32}=11.14$, $p=0.002)$ and $\operatorname{HbA} 1 \mathrm{c}\left(\mathrm{F}_{1,32}=12.75, \mathrm{p}=0.001\right)$ levels were detected in $\mathrm{m} .3243 \mathrm{~A}>\mathrm{G}$ patients compared to controls, after accounting for age, gender and BMI (body mass index). The Barthel index was, except for one patient with a score of 16 (i.e. moderate to good self-supporting), maximal. Scores for the NMDAS ranged between 0 and 26 with a median score of $8.5(\mathrm{IQR}=4-13)$.

Table. 4.2 | Main characteristics of healthy controls and m.3243A>G patients.

\begin{tabular}{l|l|l|l}
\hline Demographics & & & p-value \\
\hline Age, $\mathrm{yr}$ & $38.40(14.24)$ & $41.23(10.29)$ & 0.487 \\
\hline Sex, \% women & 73.3 & 81.8 & 0.538 \\
\hline BMl, $\mathrm{kg} / \mathrm{m}^{2}$ & $24.43(4.24)$ & $23.04(3.59)$ & 0.289 \\
\hline Education, scale & $5.20(1.21)$ & $5.09(0.92)$ & 0.838 \\
\hline Glucose status & & & \\
\hline Fasting gluose, $\mathrm{mmol} / \mathrm{L}$ & $4.99(0.45)$ & $6.56(1.70)$ & $0.001 \dagger$ \\
\hline HbA1c, \% & $5.02(0.64)$ & $6.55(1.24)$ & $<0.001 \dagger$ \\
\hline HbA1c, IFCC & $31.20(7.21)$ & $45.36(12.40)$ & $<0.001 \dagger$ \\
\hline & & &
\end{tabular}




\begin{tabular}{|c|c|c|c|}
\hline & Controls $(n=15)$ & $m .3243 A>G(n=22)$ & p-value \\
\hline \multicolumn{4}{|c|}{ Clinical relevant symptoms } \\
\hline \multicolumn{4}{|l|}{ Mutation load } \\
\hline UECs, \% & 0 & $53.14(26.09)$ & - \\
\hline Blood, \% & 0 & $20.23(11.40)$ & - \\
\hline Barthel index & - & $19.82(0.83)$ & - \\
\hline NMDAS & - & $8.50(4-13)$ & - \\
\hline Number of symptoms & 0 & $3.64(2.46)$ & - \\
\hline \multicolumn{4}{|c|}{ See Fig. $4.1 \mathrm{C}$ for more details } \\
\hline \multicolumn{4}{|c|}{ Cognitive performance } \\
\hline MMSE & $29.13(1.30)$ & $28.27(2.47)$ & 0.226 \\
\hline LDST, z-score & $0(1.0)$ & $-1.08(2.18)$ & 0.083 \\
\hline \multicolumn{4}{|l|}{ Stroop, z-score } \\
\hline Words only & $0(1.0)$ & $0.62(1.35)$ & 0.054 \\
\hline Colours only & $0(1.0)$ & $0.95(1.63)$ & 0.081 \\
\hline Words and colours & $0(1.0)$ & $1.40(2.89)$ & 0.127 \\
\hline \multicolumn{4}{|l|}{ 15-WLT, z-score } \\
\hline Total & $0(1.0)$ & $-0.38(1.04)$ & 0.282 \\
\hline Recall & $0(1.0)$ & $0.01(0.99)$ & 0.973 \\
\hline Recognition & $0(1.0)$ & $-0.84(3.01)$ & 0.310 \\
\hline
\end{tabular}

Values represent mean ( \pm S.D.) if not stated otherwise. Abbreviations: $\mathrm{BMI}=$ body mass index; $\mathrm{UEC}=$ urinary epithelial cells; NMDAS = Newcastle Mitochondrial Disease Adult Scale; MMSE = mini-mental state examination; LDST = letter-digit substitution task; 15 -WLT = 15-words learning task. ${ }^{1}$ Educational scale ranges from 1 (no education) to 8 (university). †ANOVA, corrected for age, gender and BMI.

\subsection{1. | m.3243A>G clinical characteristics}

The mutation load in blood was significantly correlated with the mutation load in UECs (Pearson correlation, $r_{18}=0.757, p<0.001$, see Fig. 4.1A) and was on average $2.95( \pm 1.40)$ times lower than in UECs. The median m.3243A $>$ G percentage in blood of $23 \%$ was used to subdivide patients in $\mathrm{P}_{\text {low }}(<23 \%$, filled dots in Fig. $4.1 \mathrm{~A})$ or $\mathrm{P}_{\text {high }}(\geq 23 \%$, empty dots). Correlation analyses revealed that both the mutation loads in blood $\left(r_{18}=0.473\right.$, $p=0.035$, see Fig. 4.1B) and UECs ( $r_{18}=0.550, p=0.012$, not shown) are positively correlated (after correcting for age and gender) with the NMDAS score. Increasing mutation load in blood, not UECs, was correlated with worse cognitive test performances for the LDST (Pearson correlation, $\left.r_{18}=-0.745, p=0.005\right)$ and Stroop tasks $\left(r_{18}=0.599, p=0.025\right.$, not shown), corrected for age, gender and education. 


\section{CHAPTER 4}
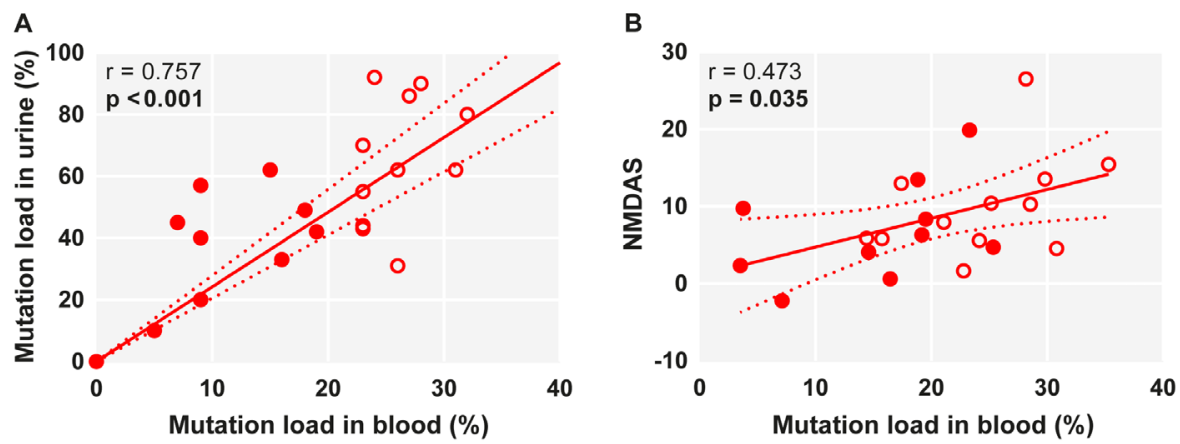

C

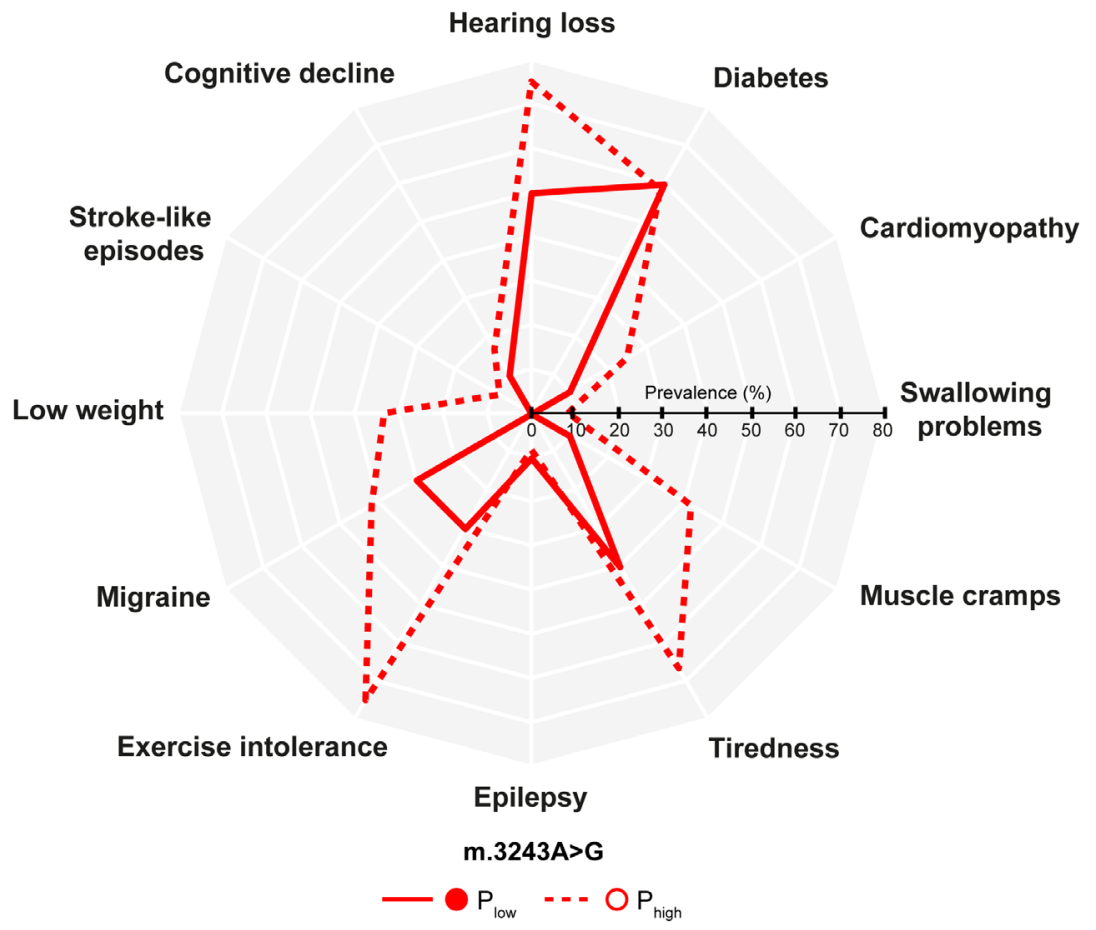

Fig. 4.1. | Correlation analysis between clinical and genetic data. Scatter plots show the correlation between m.3243A>G mutation load (\%) measured in UECS and blood (A) and the age- and gender-adjusted correlation between mutation load in blood and NMDAS score (B). Filled and empty dots represent $P_{\text {low }}$ and $P_{\text {high }}$ patients, respectively. Solid lines represent the best fit $\pm 95 \% \mathrm{Cls}$. Boldface $p$-values indicate a significant correlation. Spiderplot visualizes the prevalence of each symptom within both the $P_{\text {low }}$ (solid line) and $P_{\text {high }}$ (dashed line) m.3243A>G patient groups (C). Diabetes was true in case of known history from physician and HbA1c (IFCC) level higher than 45. Low weight was true in case of a BMI lower than 18.50. 
Hearing loss was the most prevalent observed symptom within the m.3243A $>\mathrm{G}$ patient group ( $P_{\text {low }}$ vs. $P_{\text {high }}: 50$ vs. $75 \%$ ), as can be observed in Fig. 4.1C. This was followed by diabetes mellitus (60 vs. $58.3 \%$ ), exercise intolerance (30 vs. $75 \%$ ) and tiredness (40 vs. $66.7 \%$ ). Migraine (30 vs. $41.7 \%$ ) and muscle cramps (10 vs. $41.7 \%$ ) are moderately observed, while cardiomyopathy (10 vs. $25 \%$ ), underweight (0 vs. $33.3 \%$ ), cognitive decline (10 vs. 16.7\%), epilepsy (10 vs. 8.3\%), swallowing problems and SLEs (both 0 vs. $8.3 \%$ ) are only observed in few patients. There was a significantly higher prevalence of exercise intolerance (Pearson $X^{2}$ test, $p=0.035$ ) in the $P_{\text {high }}$ group compared to the $P_{\text {low }}$ group.

\subsection{2. | Global brain morphology}

Example quantitative $T_{1}, T_{2}{ }^{*}$ and CBF maps for a control subject and an m.3243A>G patient of comparable age (66 vs. 67 yrs. old) are depicted in Fig. 4.2 across several axial slices. Note that, because we acquired quantitative MRI parameters, we can choose the same absolute scale for all subjects allowing us to examine quantitative MRI differences in addition to local contrast differences, typically indicative of lesions and atrophy. Clear signs of WMLs (see dashed blue box) were visible in both the patient's $\mathrm{T}_{1}$ and $\mathrm{T}_{2}{ }^{*}$ maps and were characterized by increased longitudinal $\left(T_{1}\right)$ and effective transverse $\left(\mathrm{T}_{2}{ }^{*}\right)$ relaxation times. In addition, a larger sulcal cerebral spinal fluid (CSF) volume (i.e. increased space between neighboring gyri) was visible for the patient, suggesting gray and/or white matter volume loss. In addition, with respect to the cerebellum, larger inter-folial spaces were visible for several patients (not shown). Possible differences regarding (sub)cortical perfusion (bottom row) were more difficult to detect visually and needed quantitative analysis. Therefore, we examined the normalized $\mathrm{T}_{1}, \mathrm{~T}_{2}{ }^{*}$ and CBF distributions for both CGM and WM tissue. Both CGM and WM $\mathrm{T}_{1}$ showed a significant increase as a function of mutation load (see Supplementary Fig. 4.1).

The total "global" volume (in $\mathrm{mm}^{3}$, including left and right hemispheres, corrected for eTIV) was computed for each structure (cGM, ventricles, subcortical GM and cerebellar GM, see Figs. 4.3A-D, respectively) to characterize the morphological differences between groups. WM and cerebellar WM volumes are shown in Supplementary Figs. $4.2 A$ and $B$, respectively. Significantly different brain volumes were detected across groups (MANOVA, Wilks' Lambda $=0.438, \mathrm{~F}_{12,54}=2.298, \mathrm{p}=0.019$ ). Here, the $\mathrm{CGM}$ as well as the WM volume were significantly different between groups $\left(F_{2,32}=7.783, p=0.002\right.$ and $F_{2,32}=5.090, p=0.012$, respectively). Similarly, group differences were observed for the ventricles $\left(\mathrm{F}_{2,32}=4.101, \mathrm{p}=0.026\right)$ and subcortical $\left(\mathrm{F}_{2,32}=5.670, \mathrm{p}=0.008\right)$ and cerebellar $\mathrm{GM}\left(\mathrm{F}_{2,32}=8.506, \mathrm{p}=0.001\right)$ volumes, but not for the cerebellar WM volume. Significant differences were only found when comparing the control subjects with the $P_{\text {high }}$ group 


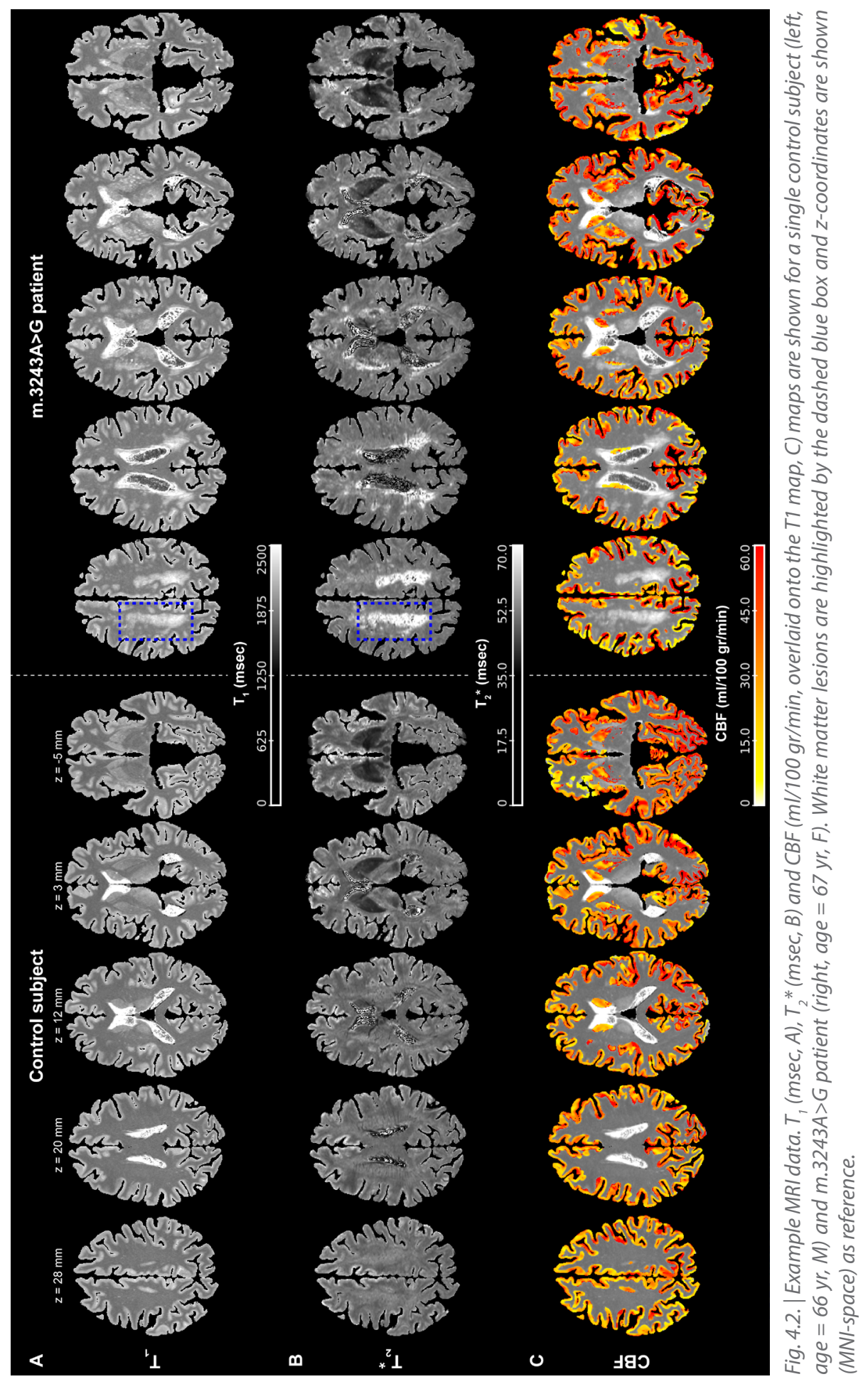




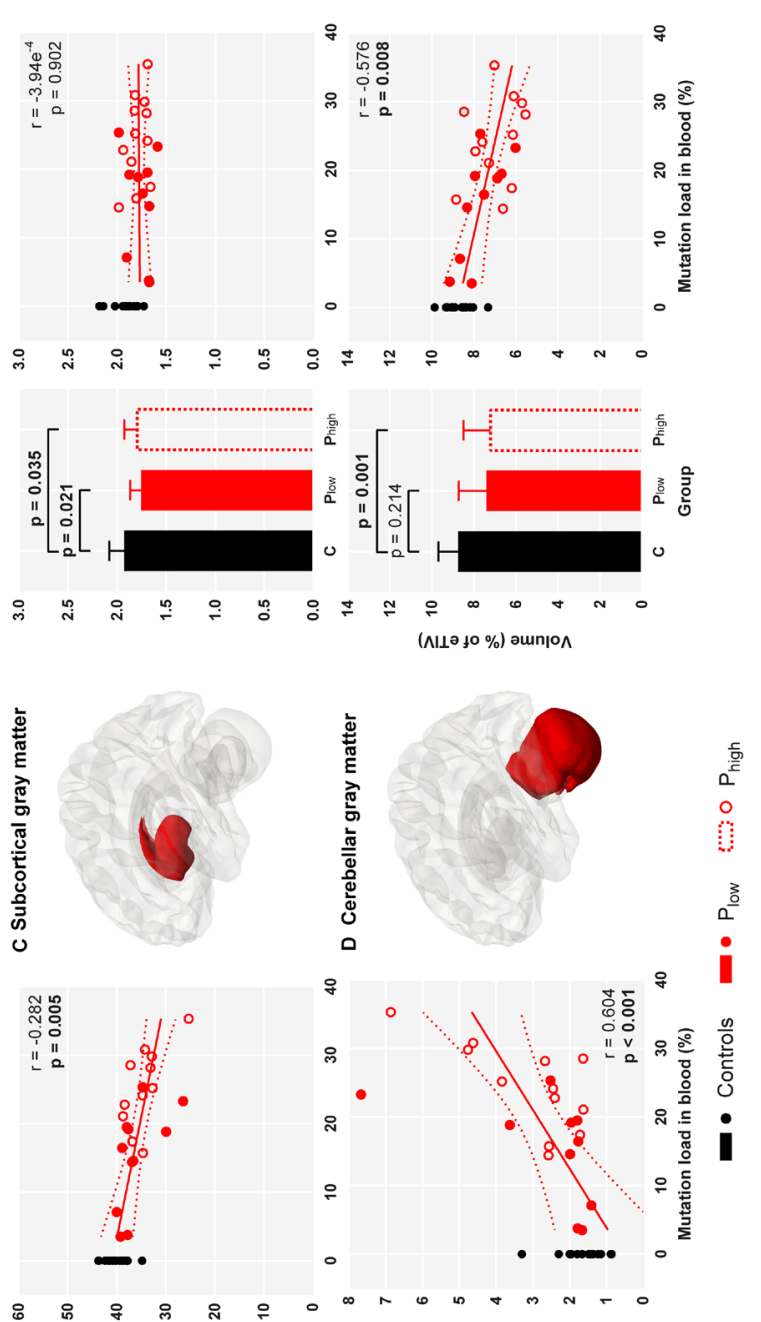

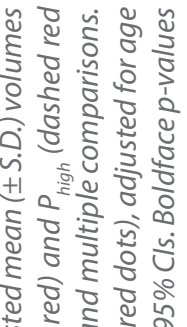

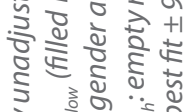

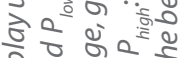

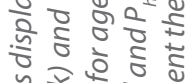

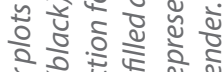

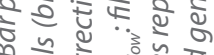

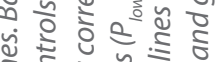

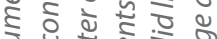

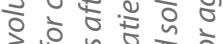

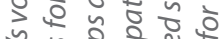

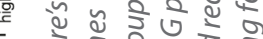

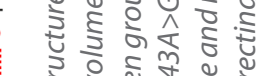

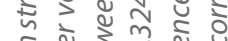

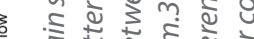

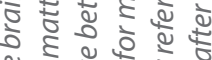
₹ ㄷำ

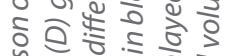

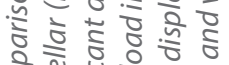

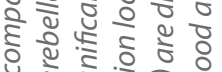
눈

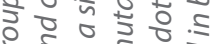
के ธ

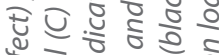
帘灾.

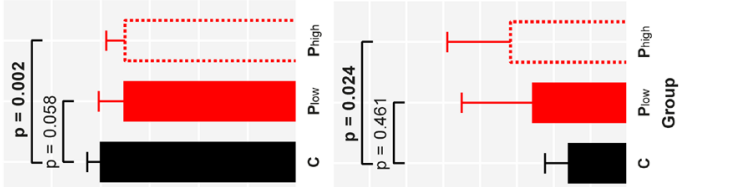

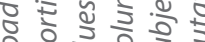
을 월 ऽิ जิ ह نِ

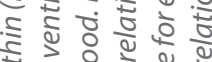

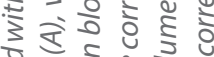

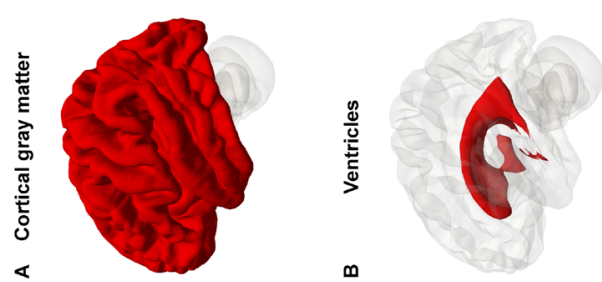

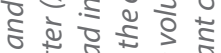

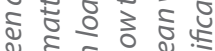

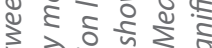

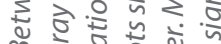
으의원응

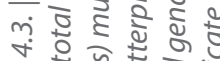
눈ㅎำ 


\section{CHAPTER 4}

$A$

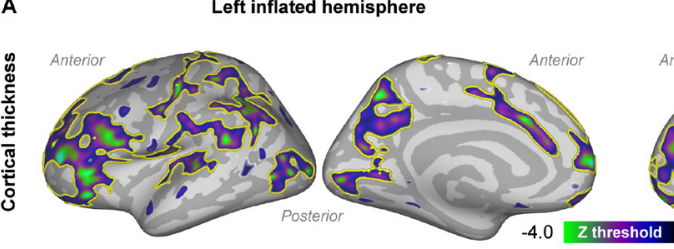

B
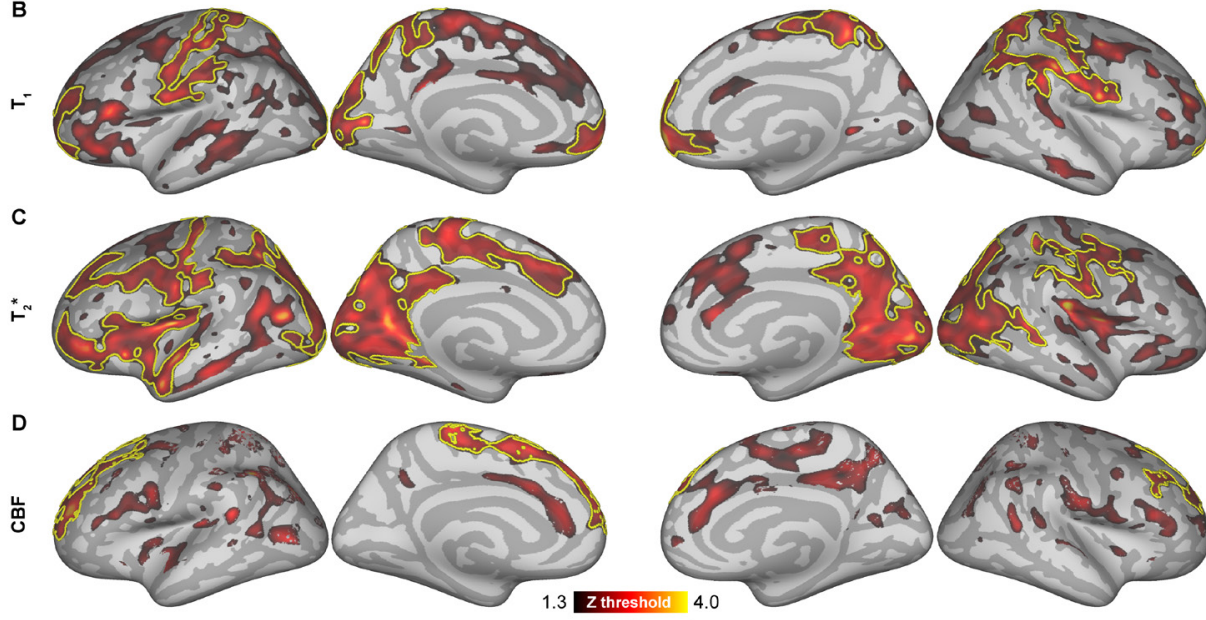

Significant clusters
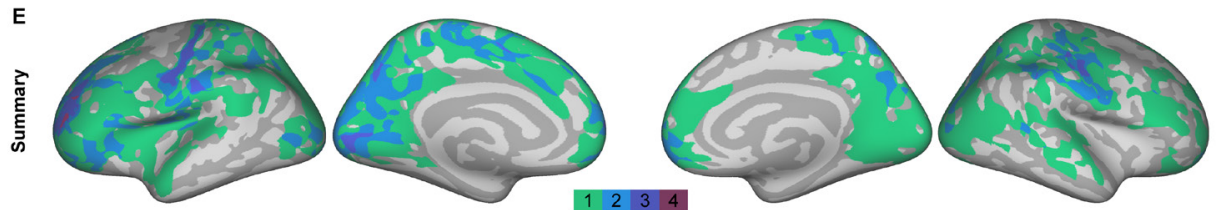

\# of significant modalities

Fig. 4.4. | Surface-based MRI data analysis. Vertex-wise linear regression analyses between mutation load in blood and cortical thickness $(A), T_{1}(B), T_{2}^{*}(C)$ and $C B F(D)$ within the $m .3243 A>G$ patients. Statistical z-maps, overlaid onto the left and right inflated (unfolded) surface reconstructions, show the vertices where cortical thickness (negatively), $T_{1}, T_{2}^{*}$ or CBF (positively) correlated with mutation load (after correction for age and gender). Significant clusters that remained after cluster-wise multiple comparison correction are delineated by yellow borders. Clusters were summarized across parameters by color-coding (1, green, to 4, purple) vertices based on the number of significant clusters (E).

( $p=0.002$ for $c G M, p=0.010$ for $W M, p=0.024$ for the ventricles, $p=0.035$ for subcortical $G M, p=0.001$ for cerebellar GM) but not with the $P_{\text {low }}$ group, except for the subcortical GM $(p=0.021)$. Significant correlations were observed between mutation load and each structure's volume (Pearson correlation, $r_{18}=-0.282, p=0.005$ for $c G M, r_{18}=-0.228$, $\mathrm{p}=0.024$ for $\mathrm{WM}, \mathrm{r}_{18}=-0.604, \mathrm{p}=0.008$ for the ventricles and $\mathrm{r}_{18}=-0.074, \mathrm{p}=0.008$ for cerebellar GM) across patients, except for the subcortical GM and cerebellar WM. Note that we did not correct for fasting glucose status, as this did not significantly affect the structures' volume within the patient group. Finally, no differences were observed across groups with regards to eTIV. 


\subsection{3. | Fine-scale cortical morphology, microstructure and perfusion}

Several cortical ROI clusters of vertices showed a significant (cluster-wise p-value of $<0.01$ ) negative correlation between cortical thickness and mutation load in blood, see Fig. 4.4A (delineated by yellow lines). In general, the clusters encompassed regions predominantly within (respective to the FreeSurfer's annotation) the middle frontal gyri, parietal (including precuneus) and superior temporal lobes. Strikingly, no significantly correlated clusters remained after multiple comparison correction when repeating the same analyses using mutation load in UECs. Similar analyses were performed using $T_{1}, T_{2}{ }^{*}$ or CBF instead of cortical thickness (see Figs. 4.4B-D, respectively). $T_{1}$ and $T_{2}{ }^{*}$ increased as a function of mutation load (cluster-wise $p$-value of $<0.05$ ) in pre- and post-central sulci, as well as in the occipital lobes, precuneus and insula for $\mathrm{T}_{2}{ }^{*}$. Increasing CBF was found in the superior frontal sulcus. To visualize the overlap of the findings using the different MRI modalities, significant clusters (vertex-value $=1$ ) were summed and color-coded, ranging between 1 (green)-4 (purple), see Fig. 4.4E. Most overlap was observed near the central sulci, frontal gyri, occipital lobes and precuneus.

Z-scores were computed per cluster for each parameter and subsequently averaged to compare across groups. Group-wise averages revealed a significant lower cortical thickness in the m.3243A $>G$ patients (including both $P_{\text {low }}$ and $P_{\text {high }}$ patients, ANOVA, $F_{1,33}=5.646, p=0.023$ ) compared to controls (see Fig. 4.5A). No significant differences were observed across groups for the $T_{1}, T_{2}{ }^{*}$ and CBF data (see Figs. 5B-D), but compared to the controls, lower $\mathrm{T}_{1}$ was observed for $\mathrm{P}_{\text {low' }}$ while a slightly higher $T_{1}$ is observed for $P_{\text {high }}$. A similar, but less apparent, pattern was observed for the $\mathrm{T}_{2}{ }^{*}$ and CBF data.

Analogous to $\mathrm{CGM}$, subcortical (i.e. alongside the CGM clusters) WM

Fig. 4.5. | Between group comparison of significant clusters. Dot plots display unadjusted mean ( $\pm S . D$ ) CGM thickness (A), $T_{1}(B), T_{2}^{*}(C)$ or CBF values (D), across corresponding significant clusters in Fig. 4.4, for controls (black), $P_{\text {low }}$ (filled red) and $P_{\text {high }}$ (empty red dots). Boldface $p$-values indicate a significant difference between groups after correction for age, gender and multiple comparisons.

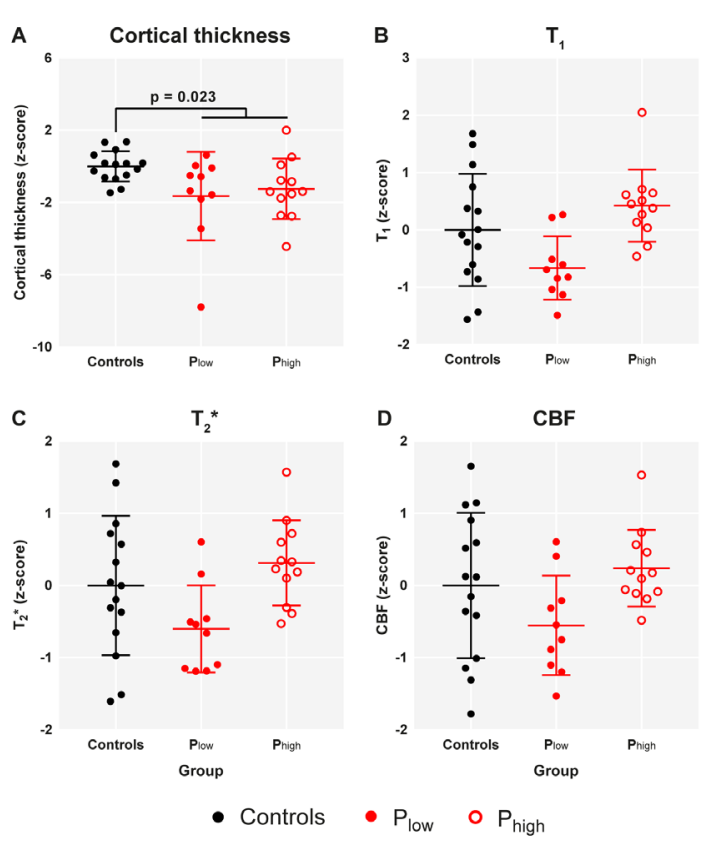




\section{CHAPTER 4}

$\mathrm{T}_{1}$ and $\mathrm{T}_{2}{ }^{*}$ were compared across groups (see Supplementary Fig. 4.2A). WM $\mathrm{T}_{1}$ and $\mathrm{T}_{2}{ }^{*}$ were not significantly different across groups. However, $\mathrm{WM}_{2}{ }^{*}$ increased with mutation load in blood within the m.3243A $>$ G patients (Pearson correlation, $r_{18}=0.454$, $p=0.044)$. Similarly, a trend was observed for $\mathrm{WMT}_{1}\left(r_{18}=0.421, p=0.065\right)$.

Regions of interest $\mathrm{T}_{1}$ $\mathrm{T}_{2}^{*}$
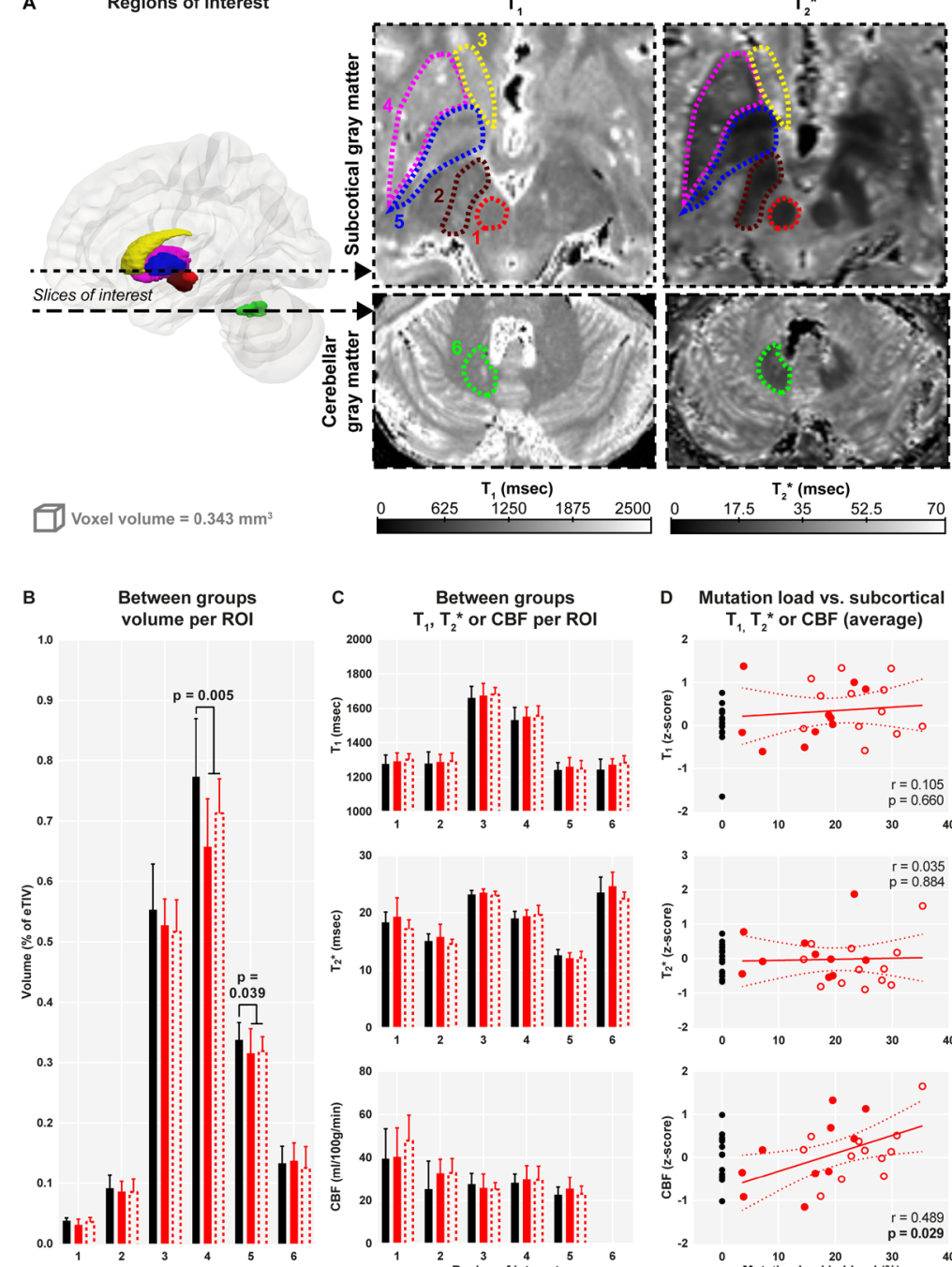

D Mutation load vs. subcortical $\mathrm{T}_{1}, \mathrm{~T}_{2}{ }^{*}$ or CBF (average)
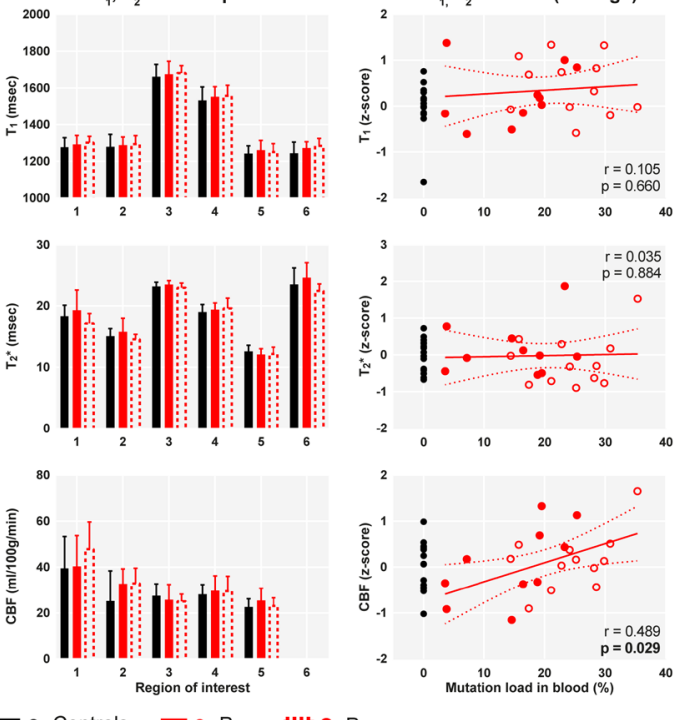

Fig. 4.6. Between and within group comparison of subcortical structures. $R O I s(1=R N, 2=S N, 3=C N$, $4=P u, 5=G P$ and $6=D N$ ) are displayed schematically in A. Mean subcortical gray matter volume (B) and $T_{1}, T_{2}^{*}$ and CBF (C) are compared across groups (controls: filled black, $P_{\text {low }}$ : filled red and $P_{\text {high }}$ : dashed red bars) per ROI. Scatterplots display the correlation between mean (z-scored relative to controls and averaged across ROIs) $T_{1}, T_{2}^{*}$ and CBF vs. mutation load in blood (D). Mean values for controls (black dots) are displayed for reference and red solid lines represent the best fit $\pm 95 \% \mathrm{Cls}$. Boldface $p$-values indicate a significant correlation between mutation load in blood and cortical thickness, $T_{1}, T_{2}^{*}$ or CBF after correction for age and gender. 


\subsection{4. | Subcortical and cerebellar morphology, microstructure and perfusion}

Similar as for the $\mathrm{CGM}$, subcortical GM volume, $\mathrm{T}_{1}, \mathrm{~T}_{2}{ }^{*}$ and $\mathrm{CBF}$ were compared across groups and as a function of mutation load within patients. Reduced subcortical GM volume was observed for both the $\mathrm{P}_{\text {low }}$ and $\mathrm{P}_{\text {high }}$ groups compared to the controls, but no effect of mutation load (see Fig. 4.3D). Group-averaged volumes for each subcortical structure (see Fig. 4.6A) individually are plotted (see Fig. 4.6B) and highlights significantly reduced volume in the m.3243A $>\mathrm{G}$ patients compared to controls (MANOVA, Wilks' Lambda $=0.616, p=0.025)$ for the Pu $\left(F_{1,33}=8.959, p=0.005\right)$ and $G P\left(F_{1,33}=4.621\right.$, $p=0.039$ ). No clear microstructural changes, i.e. between groups or as function of mutation load, in the subcortical structures were present. However, several patients showed signs of spongiotic lesions in the Pu and GP that were characterized by high $\mathrm{T}_{1}$ and $\mathrm{T}_{2}{ }^{*}$, locally (see for example Fig. 4.6A for one subject). CBF increased with mutation load (Pearson correlation, $r_{18}=0.489, p=0.029$ ).

More pronounced effects were observed for cerebellar GM (see Fig. 4.7). Here, $T_{1}$ was significantly higher for $m .3243 \mathrm{~A}>\mathrm{G}$ (ANOVA, $F_{1,33}=12.947, \mathrm{p}=0.001$ ) and especially for the $P_{\text {high }}$ group compared to controls $\left(F_{2,32}=9.915, p<0.001\right.$, see Fig. 4.7A, top row). Moreover, $T_{1}$ increases as a function of mutation load $\left(r_{18}=0.485, p=0.030\right.$, see Fig. 4.7B). In contrast, $\mathrm{T}_{2}{ }^{*}$ was comparable across groups and did not correlate with mutation load. Similar but fewer significant findings were shown for cerebellar WM (see Supp. Fig. 4.2B).

Fig. 4.7.|Between and within group comparison of cerebellar GM. Dot plots in A display unadjusted mean ( \pm S.D.) cerebellar GM $T_{1}$ (top) or $T_{2}^{*}$ (bottom) values for controls (black), $P_{\text {low }}$ (filled red) and $P_{\text {high }}$ (empty red dots). Boldface $p$-values indicate a significant difference between groups after correction for age, gender and multiple comparisons. Scatterplots in B show the correlation between $T_{1}$ or $T_{2}^{*}$ and mutation load in blood for $m .3243 A>g$ patients $\left(P_{\text {low }}\right.$ : filled and $P_{\text {high }}$ : empty red dots), adjusted for age and gender. Mean $T_{1}$ or $T_{2}^{*}$ for each control subject (black dots) are displayed for reference and red solid lines represent the best fit $\pm 95 \% \mathrm{Cls}$. Boldface $p$-values indicate a significant correlation between mutation load in blood and cerebellar $T_{1}$ or $T_{2}{ }^{*}$ after correction for age and gender.

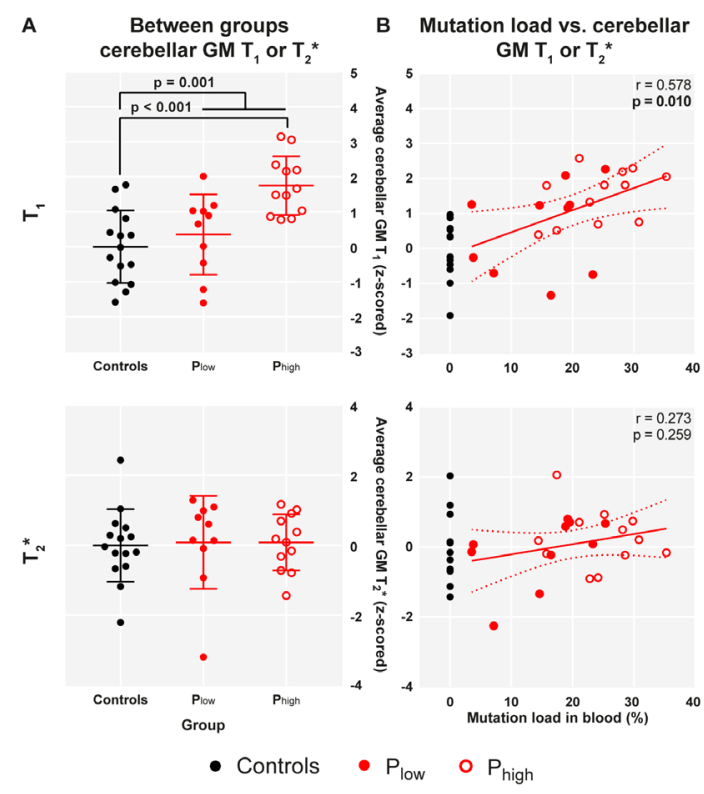




\section{CHAPTER 4}

\section{4. | DISCUSSION}

Mitochondrial dysfunctioning, either induced by acquired damage throughout life (such as by nucleases and reactive oxygen species) and/or by de novo or maternally inherited germline variations of the mtDNA, has been consistently linked to neurodegeneration (Keogh and Chinnery, 2015). While several mtDNA mutations are known to affect the brain (Reeve et al., 2008), the current study focused on the effects of the m.3243A>G genotype (i.e. mutation load) on the brain tissue using multiple parametric maps acquired using $7 \mathrm{~T} \mathrm{MRI}$ and compared these data to values of healthy controls.

\subsubsection{Mutation load and clinical characteristics}

In the current study, we obtained the mutation loads in both UECs and blood. Despite the mutation load in blood (leukocytes) being significantly lower compared to that in UECs, albeit comparable to that observed in earlier studies (Shanske et al., 2004; Whittaker et al., 2009; Fayssoil et al., 2017), both measures strongly correlated (Frederiksen et al., 2006; de Laat et al., 2012). It is known that the mutation load in blood decreases over lifetime with a constant rate of $1.4 \%$ per year (Rahman et al., 2001), while the mutation load in UECs is considered to be more consistent with age. Here, the loss of the m.3243A $>G$ mutation in blood presumably originates from a selection against pathogenic mtDNA mutations in a stem cell population (Rajasimha et al., 2008).

Hearing loss was the most prevalent symptom within the current study population, followed by exercise intolerance and diabetes mellitus. This matches with the MELAS and MIDD-like phenotypes associated with the m.3243A>G mutation and is in line with previous findings, reporting comparable numbers across their study cohorts (de Laat et al., 2012; Nesbitt et al., 2013; Fayssoil et al., 2017). In addition, we found a significant correlation between mutation load (in blood and UECs) and NMDAS score, as also shown in previous studies (Whittaker et al., 2009; de Laat et al., 2012). The observed differences between the $\mathrm{P}_{\text {low }}$ and $\mathrm{P}_{\text {high }}$ groups in terms of symptoms prevalence, especially in the myopathy-related symptoms (e.g. exercise intolerance), can partly be explained by the "mitochondrial threshold effect" (Rossignol et al., 2003). The significant correlation between the proportion of apoptotic muscle fibres and the proportion of mutant mtDNA exemplifies this hypothesis (Aure et al., 2006). It is known that the mitochondrial copy number can compensate and can be increased by physical exercise, implying the mutation load is not the only factor involved. Like muscle cells, neurons are post-mitotic and therefore are likely to behave similarly. Comparable mutation loads were detected across several brain regions to those in skeletal muscle. 
However, it is not known how the mutation load in neurons develops over lifetime (Betts et al., 2006). We have found that the mutation load in blood is a better marker for cognitive performances and brain functioning in $\mathrm{m} .3243 \mathrm{~A}>\mathrm{G}$ patients than the mutation load in UECs, even though both mutation loads are correlated to each other. The reason for this discrepancy is unknown, but may be specific to the age-range of the current patient population.

\subsection{2. | Brain morphological markers of the m.3243A>G mutation}

The current results present strong evidence of a mutation load effect within the patient population on global brain volume, in addition to widespread lower brain volume in the patients compared to controls. While patients with a low mutation load are characterized by normal-appearing brains, stronger brain atrophy is observed in patients with higher mutation load. This was especially apparent in the cortical and cerebellar GM, but not for the subcortical structures, which is in accordance with biochemical analyses of post-mortem brains (Sparaco et al., 2003). Strongest local cGM atrophy and reduced cortical thickness as a function of m.3243A $>\mathrm{G}$ mutation load was observed in regions linked to: (i) auditory processing (superior temporal cortices), (ii) attentional control (middle frontal and parietal gyri and anterior cingulate cortices), (iii) sensorimotor network (central sulci and neighboring gyri) and (iv) default mode network (precuneus). These latter two structures were also observed by a recent study that used voxel-based morphometry to characterize morphological differences between m.3243A>G patients and healthy controls (Tsujikawa et al., 2016). Notably, significant local correlations, after correction for multiple comparisons, between cortical thickness and mutation load were only found for values from the blood.

The neuropathological effects of the mutation load in some brain areas, but not in others, signify the differential abilities of neurons to survive respiratory chain deficiencies (Betts et al., 2006). More work has to be performed, particularly using invasive cellular methods, to establish the link between mitochondrial dysfunction and macroscopic brain changes, as detected with non-invasive MRI. Nevertheless, the observed brain patterns can be readily related to the different clinical symptoms often observed in m.3243A>G patients, such as the currently high prevalence of hearing impairment. Since auditory brainstem responses are often preserved, it is likely of cochlear origin (Kullar et al., 2016). In addition, the severity of the hearing loss correlates with the mutation load, being worse in patients with a higher mutation load (Chinnery et al., 2000). Therefore, decreasing cortical thickness and atrophy in the regions linked to auditory processing (auditory cortex, planum temporale and superior temporal sulcus and gyrus) with increasing m.3243A $>$ G mutation load, are most likely caused by 


\section{CHAPTER 4}

impaired cochlear functioning (Syka, 2002; Formisano et al., 2008; Eckert et al., 2012).

Even though the cortical thinning in the auditory regions may predominantly occur after cochlear damage, the morphological differences in the other affected brain regions may be induced through direct interferences of the mutation on neuronal integrity through, for example, neuronal hyperexcitability (lizuka et al., 2002; lizuka et al., 2003). Impaired mitochondrial protein (i.e. OXPHOS subunits) synthesis due to a defective tRNA ${ }^{\text {Leu(UUR) }}$ has been shown in the cerebral and cerebellar cortex of post-mortem m.3243A>G brains (Sparaco et al., 2003). Here, changes were mostly found in the intermediate layers of CGM regions, with a higher degree of cellular dependence on oxidative metabolism, and in the cerebellar Purkinje cells, respectively. As such, neuronal changes may occur especially in brain regions characterized by a high (baseline) energy demand and/or functional connectivity or cerebellum (i.e. metabolic hypothesis), depending on the mutation load similar to that observed in skeletal muscle cells (Aure et al., 2006). These high energy consuming brain areas include regions related to the default mode, dorsal attention and sensorimotor networks (Tomasi et al., 2013), in agreement with our findings of atrophy in these regions, such as in the precuneus, middle frontal gyrus and central sulcus (somatosensory and motor cortices), respectively. The cortical thinning observed in regions associated with attentional control/executive functioning, such as the middle frontal and parietal gyri and anterior cingulate cortices (Milham et al., 2002), are consistent with behavioral symptoms, e.g. patients with a higher mutation load performed worse on the Stroop task. In particular, OXPHOS deficits-susceptible inhibitory interneurons may play a prominent role in the cognitive dysfunctioning observed in mitochondrial disease patients (Finsterer, 2012; Lax et al., 2016).

Comparable to the cGM atrophy, cerebellar GM atrophy was most pronounced in the high mutation load group compared to patients with a lower mutation load, quantified by a volume loss or illustrated by larger inter-folial spaces. Previously, studies have shown that the cerebellum is highly vulnerable to mitochondrial dysfunctioning (Scaglia et al., 2005) due to respiratory chain deficiencies of complex I and IV, leading to cerebellar atrophy by Purkinje and granule cell loss (Sparaco et al., 2003; Lax et al., 2012). This is consistent with the fact that cerebellar ataxia is commonly described in mtDNA patients (de Laat et al., 2012; Lax et al., 2012). As for cerebellar GM, smaller subcortical volume was observed for the patients compared to the healthy controls, in particular in the Pu and GP (i.e. lentiform nucleus), structures especially involved in processing motor information (Middleton and Strick, 2000). The volume loss correlates with the described neuronal vacuolization (i.e. spongiosis) in both these structures for 
several patients, potentially resulting from abnormalities within the vascular smooth muscle and endothelial cells (i.e. vascular hypothesis, Betts et al. (2006)) or from astroglial pathogenesis (van der Knaap et al., 2012; Bugiani et al., 2017). However, in contrast to the cortical and cerebellar GM, the mutation load did not affect the degree of subcortical atrophy. This may implicate that the causal mechanism is different compared to that underlying cortical atrophy (e.g. vascular vs. metabolic hypotheses). Generally, by taking together the cortical atrophy in sensorimotor regions, subcortical (i.e. lentiform nucleus) and cerebellar GM volume losses, the current results suggest a global brain-wide impaired motor functioning (Seidler et al., 2015). These changes are excellent brain correlates to some of the symptoms often observed in m.3243A>G and mitochondrial disease patients, such as cerebellar (and/or sensory) ataxia and gait instability (de Laat et al., 2012).

\subsection{3. | Cortical microstructure- and metabolic-related markers of the m.3243A>G mutation}

Besides macroscopic morphological differences, $\mathrm{T}_{1}, \mathrm{~T}_{2}{ }^{*}$ and $\mathrm{CBF}$ significantly varied (i.e. increased) as a function of mutation load in several cGM regions. Differences in quantitative $T_{1}$ and $T_{2}{ }^{*}$ across the brain are predominantly attributed to varying myelin and iron content between brain structures and regions (Stuber et al., 2014). In line with earlier work that showed delayed or decreased myelination in patients affected by respiratory chain defects (Dinopoulos et al., 2005; Sofou et al., 2013; Lax et al., 2016), positive correlations between $T_{1}$ and $T_{2}{ }^{*}$ values with mutation load within the patient population were observed, in particular in the primary- and heavily-myelinated regions of the CGM, including the somatosensory- and motor cortices (Haast et al., 2016), but also in cerebellar GM. In general, increased $T_{1}$ and $T_{2}{ }^{*}$ implicate lower levels of myelin (Stuber et al., 2014). Decreased myelin synthesis, due to the defective cellular energy production during the critical time of myelin development in early life, may have resulted in the increasing $T_{1}$ and $T_{2}{ }^{*}$ with mutation load. Furthermore, we observed higher CBF in the subcortical structures, as well as in the superior frontal gyrus, for patients with a higher mutation load. These effects could be induced (i) by the altered vascular functionality (e.g. related to the subcortical spongiosis and vascular abnormalities), (ii) by a compensatory mechanism for the impaired neuronal energy production or (iii) as an attempt to reduce the increased brain lactate (or other potentially harmful compounds) levels, as argued by Rodan et al. (2015).

However, when the patient populations are compared against controls, surprisingly, the metabolic-, but also microstructural-related characteristics do not follow the expected linear change as a function of mutation load, but a U-shaped dependency 


\section{CHAPTER 4}

across groups. That is, MRI values over the entire cortex of $\mathrm{P}_{\text {high }}$ patients and healthy subjects are similarly different than those of the $P_{\text {low }}$ patients. Two hypotheses could explain this observed pattern: i) the dip in cortical CBF, $T_{1}$ and $T_{2}^{*}$ values for the $P_{\text {low }}$ patients may signify that certain compensatory mechanisms are initiated only in the $P_{\text {high }}$ patients (i.e. mitochondrial threshold effect). That is, for the $P_{\text {low }}$ group, there is only a mild loss in e.g. myelination, which does not evoke/necessitate activation of local molecular pathways to compensate for this loss. However, for the $\mathrm{P}_{\text {high }}$ group, compensatory mechanisms are necessary to maintain functioning of the respective brain area. In fact, delayed myelination in the $P_{\text {high }}$ group, as described above, may have led to global myelination levels similar to control ranges, as suggested by the correspondence between the range of $\mathrm{T}_{1}$ and $\mathrm{T}_{2}{ }^{*}$ values observed in the group-wise comparison between controls and patients, confirming earlier claims (Dinopoulos et al., 2005; Sofou et al., 2013). Alternatively, ii) $T_{1}, T_{2}^{*}$ and brain metabolic changes have many biochemical and microscopic contributions, which do not have to follow the same dependency on mutation load. For example, $\mathrm{T}_{1}$ increases as a function of both myelin and iron. That is, a decrease in myelin has an opposing effect on the resulting $T_{1}$ values than an increase in iron content, and the latter change is a common observation in many neurodegenerative diseases. Thus, it is reasonable to assume that myelination decreases and iron content increases as a function of mutation load but they have different dependencies on the m.3243A>G mutation load, leading to the U-shape function observed pattern for $\mathrm{T}_{1}$. In contrast to the observed cortical pattern, the increased cerebellar GM T, observed in patients compared to controls, in addition to the unchanged $\mathrm{T}_{2}{ }^{*}$, may implicate predominantly myelin loss secondary to neuronal and axonal loss (Virtanen et al., 2011; Lax et al., 2012).

Interestingly, $\mathrm{T}_{1}$ and $\mathrm{T}_{2}{ }^{*}$ differences in the subcortical structures were absent, which is indicative for subcortical biochemical changes that may be more specific for certain mtDNA mutations (Barragan-Campos et al., 2005; Bindu et al., 2015). This is in sharp contrast to previously observed subcortical $\mathrm{T}_{1} \mathrm{w}$ and $\mathrm{T}_{2} \mathrm{w}$ changes (Tschampa et al., 2013). However, it is important to note that most of the available MRI studies, if not all, employed data acquired using conventional clinical, non-quantitative MRI approaches, such as FLAIR. These do not provide quantitative markers of the underlying biochemical composition of the tissue, as the MRI signal intensity are additionally influenced by MRI sequence parameters and the hardware setup (such as transmit and receive imaging coils) (Bock et al., 2013; Lorio et al., 2016). Moreover, clinical scans typically have anisotropic voxel sizes, increasing partial voluming effects and mixing signals originating from WM and GM or spongiotic tissue and subcortical GM. Therefore, quantitative interpretation of data acquired for diagnostic purposes is challenging, as 
interpretations of such data contributions are confounded by many other MRI acquisition and physiological parameters (e.g. transmit and receive RF fields, proton density, relaxation rates, macromolecule concentrations (Haast et al., 2016)). In summary, these results exemplify the benefits of a multi-parametric MRI protocol, as they allow discerning the biochemical causes of brain changes in disease and reliable intra- and inter-subject comparisons.

\subsection{4. | Conclusions}

In conclusion, the current work utilized high-resolution MRI data acquired using 7T MRI to quantify brain changes in a representative population of m.3243A $>G$ patients. The data revealed neuroradiological (i.e. morphology, microstructure- and metabolism-related) changes in patients with varying levels of mutation load, including those with a mutation load lower than previously observed threshold levels, compared to healthy controls (see Table 4.3 for an overview).

Table 4.3 | Overview of observed brain differences between m.3243A>G patients and healthy controls, and as function of mutation load (gray columns). Only significant findings are listed. Upward and downwards faced arrows indicate an increase or decrease of the corresponding parameter, respectively, while the boxes crossed out were not quantifiable.

\begin{tabular}{l|c|c|c|c|c|c|c|c|c|c}
\hline & \multicolumn{3}{c}{ Volume } & \multicolumn{1}{c}{ Thickness } & $\mathbf{T}_{1}$ & \multicolumn{2}{c}{$\mathbf{T}_{2}^{*}$} & \multicolumn{3}{c}{ CBF } \\
Cortical GM & $\downarrow^{1}$ & $\downarrow$ & - & - & - & $\uparrow$ & - & - & - & - \\
\hline Cortical GM clusters & & & $\downarrow, 14^{2}$ & $\downarrow$ & & $\uparrow, 5$ & & $\uparrow$ & & $\uparrow, 2$ \\
\hline WM & $\downarrow$ & $\downarrow$ & & & - & $\uparrow$ & - & - & & \\
\hline Subcortical WM clusters & & & & & - & - & - & $\uparrow$ & & \\
\hline Subcortical GM & $\downarrow$ & - & & & - & - & - & - & - & $\uparrow$ \\
\hline Putamen & $\downarrow$ & - & & & - & - & - & - & - & - \\
\hline Globus pallidus & $\downarrow$ & - & & & - & - & - & - & - & - \\
\hline Cerebellar GM & $\downarrow$ & $\downarrow$ & & $\uparrow$ & $\uparrow$ & - & - & & \\
\hline Cerebellar WM & - & - & & & - & - & - & - & & \\
\hline Ventricles & $\uparrow$ & $\uparrow$ & & - & & - & & - & \\
\hline
\end{tabular}

${ }^{1}$ Significant difference between controls and $\mathrm{P}_{\text {high }}$ group only. ${ }^{2}$ Number of significant clusters, summed across hemispheres.

Interestingly, mutation load in blood compared to UECs provides a better estimate of the brain changes. The observed changes are widespread, but mainly affect GM, and are consistent with the different clinical symptoms. However, the underlying biological mechanisms and timing may vary across brain structures/regions. Our results, thus, indicate the value of quantitative MRI at 7T to determine pathological effects of the m.3243A>G mutation on brain structure and function for various mutation loads. 


\section{CHAPTER 4}

Future longitudinal quantitative MRI but also in vitro studies are necessary to track the progression of the detected MRI-based biomarkers to improve the understanding of the disease pathogenesis and clinical symptoms development. 


\section{5. | REFERENCES}

Aure, K., G. Fayet, J. P. Leroy, E. Lacene, N. B. Romero and A. Lombes (2006). "Apoptosis in mitochondrial myopathies is linked to mitochondrial proliferation." Brain 129(Pt 5): 1249-1259.

Barragan-Campos, H. M., J. N. Vallee, D. Lo, C. F. Barrera-Ramirez, M. Argote-Greene, J. Sanchez-Guerrero, ... J. Chiras (2005). "Brain magnetic resonance imaging findings in patients with mitochondrial cytopathies." Arch Neurol 62(5): 737-742.

Betts, J., E. Jaros, R. H. Perry, A. M. Schaefer, R. W. Taylor, Z. Abdel-All, . . D. M. Turnbull (2006). "Molecular neuropathology of MELAS: level of heteroplasmy in individual neurones and evidence of extensive vascular involvement." Neuropathol Appl Neurobiol 32(4): 359-373.

Bindu, P. S., H. Arvinda, A. B. Taly, C. Govindaraju, K. Sonam, S. Chiplunkar, ... K. Thangaraj (2015). "Magnetic resonance imaging correlates of genetically characterized patients with mitochondrial disorders: A study from south India." Mitochondrion 25: 6-16.

Bock, N. A., E. Hashim, R. Janik, N. B. Konyer, M. Weiss, G. J. Stanisz, .. S. Geyer (2013). “Optimizing T1-weighted imaging of cortical myelin content at 3.0 T." Neuroimage 65: 1-12.

Bugiani, M., M. Dubey, M. Breur, N. L. Postma, M. P. Dekker, T. Ter Braak, ... M. S. van der Knaap (2017). "Megalencephalic leukoencephalopathy with cysts: the Glialcam-null mouse model." Ann Clin Transl Neurol 4(7): 450-465.

Chinnery, P. F., C. Elliott, G. R. Green, A. Rees, A. Coulthard, D. M. Turnbull and T. D. Griffiths (2000). "The spectrum of hearing loss due to mitochondrial DNA defects." Brain 123 ( Pt 1): 82-92.

Chinnery, P. F., N. Howell, R. N. Lightowlers and D. M. Turnbull (1997). "Molecular pathology of MELAS and MERRF. The relationship between mutation load and clinical phenotypes." Brain 120 (Pt 10): 1713-1721.

Chomyn, A., A. Martinuzzi, M. Yoneda, A. Daga, O. Hurko, D. Johns, ... G. Attardi (1992). “MELAS mutation in mtDNA binding site for transcription termination factor causes defects in protein synthesis and in respiration but no change in levels of upstream and downstream mature transcripts." Proc Natl Acad Sci U S A 89(10): 4221-4225.

Dale, A. M., B. Fischl and M. I. Sereno (1999). “Cortical surface-based analysis. I. Segmentation and surface reconstruction." Neuroimage 9(2): 179-194.

de Laat, P., S. Koene, L. P. van den Heuvel, R. J. Rodenburg, M. C. Janssen and J. A. Smeitink (2012). "Clinical features and heteroplasmy in blood, urine and saliva in 34 Dutch families carrying the m.3243A > G mutation." J Inherit Metab Dis 35(6): 1059-1069.

Dinopoulos, A., K. M. Cecil, M. B. Schapiro, A. Papadimitriou, G. M. Hadjigeorgiou, B. Wong, ... J. C. Egelhoff (2005). "Brain MRI and proton MRS findings in infants and children with respiratory chain defects." Neuropediatrics 36(5): 290-301.

Duyn, J. H., P. van Gelderen, T. Q. Li, J. A. de Zwart, A. P. Koretsky and M. Fukunaga (2007). “Highfield MRI of brain cortical substructure based on signal phase." Proc Natl Acad Sci U S A 104(28): 11796-11801.

Eckert, M. A., S. L. Cute, K. I. Vaden, Jr., S. E. Kuchinsky and J. R. Dubno (2012). "Auditory cortex signs of age-related hearing loss." J Assoc Res Otolaryngol 13(5): 703-713.

Eggenschwiler, F., T. Kober, A. W. Magill, R. Gruetter and J. P. Marques (2012). "SA2RAGE: a new sequence for fast B1+ -mapping." Magn Reson Med 67(6): 1609-1619.

Fayssoil, A., P. Laforet, W. Bougouin, C. Jardel, A. Lombes, H. M. Becane, ... K. Wahbi (2017). “Prediction of long-term prognosis by heteroplasmy levels of the m.3243A>G mutation in patients with the mitochondrial encephalomyopathy, lactic acidosis and stroke-like episodes syndrome." Eur J Neurol 24(2): 255-261.

Finsterer, J. (2012). “Cognitive dysfunction in mitochondrial disorders." Acta Neurol Scand 126(1): 1-11.

Fischl, B., D. H. Salat, E. Busa, M. Albert, M. Dieterich, C. Haselgrove, ... A. M. Dale (2002). “Whole 


\section{CHAPTER 4}

brain segmentation: automated labeling of neuroanatomical structures in the human brain." Neuron 33(3): 341-355.

Fischl, B., M. I. Sereno, R. B. Tootell and A. M. Dale (1999). “High-resolution intersubject averaging and a coordinate system for the cortical surface." Hum Brain Mapp 8(4): 272-284.

Formisano, E., F. De Martino, M. Bonte and R. Goebel (2008). "'"Who" is saying "what"? Brain-based decoding of human voice and speech." Science 322(5903): 970-973.

Frederiksen, A. L., P. H. Andersen, K. O. Kyvik, T. D. Jeppesen, J. Vissing and M. Schwartz (2006). "Tissue specific distribution of the 3243A-> G mtDNA mutation." J Med Genet 43(8): 671-677.

Gardener, A. G., P. A. Gowland and S. T. Francis (2009). "Implementation of quantitative perfusion imaging using pulsed arterial spin labeling at ultra-high field." Magn Reson Med 61(4): 874-882.

Goto, Y., I. Nonaka and S. Horai (1990). "A mutation in the tRNA(Leu)(UUR) gene associated with the MELAS subgroup of mitochondrial encephalomyopathies." Nature 348(6302): 651-653.

Haast, R. A., D. Ivanov, E. Formisano and K. Uludag (2016). "Reproducibility and Reliability of Quantitative and Weighted T1 and T2 * Mapping for Myelin-Based Cortical Parcellation at 7 Tesla." Front Neuroanat 10: 112.

lizuka, T., F. Sakai, S. Kan and N. Suzuki (2003). "Slowly progressive spread of the stroke-like lesions in MELAS." Neurology 61(9): 1238-1244.

lizuka, T., F. Sakai, N. Suzuki, T. Hata, S. Tsukahara, M. Fukuda and Y. Takiyama (2002). “Neuronal hyperexcitability in stroke-like episodes of MELAS syndrome." Neurology 59(6): 816-824.

Ikawa, M., M. Yoneda, T. Muramatsu, A. Matsunaga, T. Tsujikawa, T. Yamamoto, ... H. Kimura (2013). "Detection of preclinically latent hyperperfusion due to stroke-like episodes by arterial spin-labeling perfusion MRI in MELAS patients." Mitochondrion 13(6): 676-680.

Ivanov, D., A. Gardumi, R. A. M. Haast, J. Pfeuffer, B. A. Poser and K. Uludag (2017). "Comparison of 3T and 7T ASL techniques for concurrent functional perfusion and BOLD studies." Neuroimage 156: 363-376.

Ivanov, D., B. A. Poser, L. Huber, J. Pfeuffer and K. Uludag (2017). “Optimization of simultaneous multislice EPI for concurrent functional perfusion and BOLD signal measurements at 7T." Magn Reson Med 78(1): 121-129.

Keogh, M. J. and P. F. Chinnery (2015). "Mitochondrial DNA mutations in neurodegeneration." Biochim Biophys Acta 1847(11): 1401-1411.

Keuken, M. C., P. L. Bazin, K. Backhouse, S. Beekhuizen, L. Himmer, A. Kandola, . . B. U. Forstmann (2017). "Effects of aging on $\mathrm{T}_{1}, \mathrm{~T}_{2}{ }^{*}$, and QSM MRI values in the subcortex." Brain Struct Funct.

Kollia, K., S. Maderwald, N. Putzki, M. Schlamann, J. M. Theysohn, O. Kraff, ... I. Wanke (2009). “First clinical study on ultra-high-field MR imaging in patients with multiple sclerosis: comparison of 1.5T and 7T." AJNR Am J Neuroradiol 30(4): 699-702.

Kullar, P. J., J. Quail, P. Lindsey, J. A. Wilson, R. Horvath, P. Yu-Wai-Man, . . P. F. Chinnery (2016). "Both mitochondrial DNA and mitonuclear gene mutations cause hearing loss through cochlear dysfunction." Brain 139(Pt 6): e33.

Lax, N. Z., J. Grady, A. Laude, F. Chan, P. D. Hepplewhite, G. Gorman, .. . D. M. Turnbull (2016). "Extensive respiratory chain defects in inhibitory interneurones in patients with mitochondrial disease." Neuropathol Appl Neurobiol 42(2): 180-193.

Lax, N. Z., P. D. Hepplewhite, A. K. Reeve, V. Nesbitt, R. McFarland, E. Jaros, ... D. M. Turnbull (2012). "Cerebellar ataxia in patients with mitochondrial DNA disease: a molecular clinicopathological study." J Neuropathol Exp Neurol 71(2): 148-161.

Li, R., H. F. Xiao, J. H. Lyu, J. J. W. D, L. Ma and X. Lou (2017). “Differential diagnosis of mitochondrial encephalopathy with lactic acidosis and stroke-like episodes (MELAS) and ischemic stroke using 3D pseudocontinuous arterial spin labeling." J Magn Reson Imaging 45(1): 199-206.

Lightowlers, R. N., R. W. Taylor and D. M. Turnbull (2015). "Mutations causing mitochondrial disease: What is new and what challenges remain?" Science 349(6255): 1494-1499. 
Lorio, S., F. Kherif, A. Ruef, L. Melie-Garcia, R. Frackowiak, J. Ashburner, ... B. Draganski (2016). "Neurobiological origin of spurious brain morphological changes: A quantitative MRI study." Hum Brain Mapp 37(5): 1801-1815.

Magistretti, P. J. and I. Allaman (2015). "A cellular perspective on brain energy metabolism and functional imaging." Neuron 86(4): 883-901.

Marques, J. P., T. Kober, G. Krueger, W. van der Zwaag, P. F. Van de Moortele and R. Gruetter (2010). "MP2RAGE, a self bias-field corrected sequence for improved segmentation and T1-mapping at high field." Neuroimage 49(2): 1271-1281.

Middleton, F. A. and P. L. Strick (2000). "Basal ganglia and cerebellar loops: motor and cognitive circuits." Brain Res Brain Res Rev 31 (2-3): 236-250.

Milham, M. P., K. I. Erickson, M. T. Banich, A. F. Kramer, A. Webb, T. Wszalek and N. J. Cohen (2002). "Attentional control in the aging brain: insights from an fMRI study of the stroop task." Brain Cogn 49(3): 277-296.

Nakada, T., H. Matsuzawa, H. Igarashi, Y. Fujii and I. L. Kwee (2008). "In vivo visualization of senile-plaque-like pathology in Alzheimer's disease patients by MR microscopy on a 7T system." $\mathrm{J}$ Neuroimaging 18(2): 125-129.

Nesbitt, V., R. D. Pitceathly, D. M. Turnbull, R. W. Taylor, M. G. Sweeney, E. E. Mudanohwo, ... R. McFarland (2013). "The UK MRC Mitochondrial Disease Patient Cohort Study: clinical phenotypes associated with the m.3243A>G mutation--implications for diagnosis and management." J Neurol Neurosurg Psychiatry 84(8): 936-938.

Norris, D. G. (2003). “High field human imaging.” J Magn Reson Imaging 18(5): 519-529.

Pfeuffer, J., P. F. van de Moortele, E. Yacoub, A. Shmuel, G. Adriany, P. Andersen, ... X. Hu (2002). "Zoomed functional imaging in the human brain at 7 Tesla with simultaneous high spatial and high temporal resolution." Neuroimage 17(1): 272-286.

Pohmann, R., O. Speck and K. Scheffler (2016). “Signal-to-noise ratio and MR tissue parameters in human brain imaging at 3, 7, and 9.4 tesla using current receive coil arrays." Magn Reson Med 75(2): 801-809.

Rahman, S., J. Poulton, D. Marchington and A. Suomalainen (2001). “Decrease of 3243 A-->G mtDNA mutation from blood in MELAS syndrome: a longitudinal study." Am J Hum Genet 68(1): 238-240.

Rajasimha, H. K., P. F. Chinnery and D. C. Samuels (2008). "Selection against pathogenic mtDNA mutations in a stem cell population leads to the loss of the 3243A-->G mutation in blood." Am J Hum Genet 82(2): 333-343.

Reeve, A. K., K. J. Krishnan and D. Turnbull (2008). "Mitochondrial DNA mutations in disease, aging, and neurodegeneration." Ann NY Acad Sci 1147: 21-29.

Rodan, L. H., J. Poublanc, J. A. Fisher, O. Sobczyk, T. Wong, E. Hlasny, . . I. Tein (2015). “Cerebral hyperperfusion and decreased cerebrovascular reactivity correlate with neurologic disease severity in MELAS." Mitochondrion 22: 66-74.

Romero, J. E., P. Coupe, R. Giraud, V.T. Ta, V. Fonov, M. T. Park, .. J. V. Manjon (2017). “CERES: A new cerebellum lobule segmentation method." Neuroimage 147: 916-924.

Rossignol, R., B. Faustin, C. Rocher, M. Malgat, J. P. Mazat and T. Letellier (2003). “Mitochondrial threshold effects." Biochem J 370(Pt 3): 751-762.

Sallevelt, S. C., J. C. Dreesen, M. Drusedau, S. Spierts, E. Coonen, F. H. van Tienen, ... H. J. Smeets (2013). "Preimplantation genetic diagnosis in mitochondrial DNA disorders: challenge and success." J Med Genet 50(2): 125-132.

Saneto, R. P., S. D. Friedman and D. W. Shaw (2008). "Neuroimaging of mitochondrial disease." Mitochondrion 8(5-6): 396-413.

Scaglia, F., L. J. Wong, G. D. Vladutiu and J. V. Hunter (2005). “Predominant cerebellar volume loss as a neuroradiologic feature of pediatric respiratory chain defects." AJNR Am J Neuroradiol 26(7): 1675-1680. 


\section{CHAPTER 4}

Schaefer, A. M., C. Phoenix, J. L. Elson, R. McFarland, P. F. Chinnery and D. M. Turnbull (2006). “Mitochondrial disease in adults: a scale to monitor progression and treatment." Neurology 66(12): 1932-1934.

Seidler, R., B. Erdeniz, V. Koppelmans, S. Hirsiger, S. Merillat and L. Jancke (2015). “Associations between age, motor function, and resting state sensorimotor network connectivity in healthy older adults." Neuroimage 108: 47-59.

Shanske, S., J. Pancrudo, P. Kaufmann, K. Engelstad, S. Jhung, J. Lu, . . . D. C. De Vivo (2004). "Varying loads of the mitochondrial DNA A3243G mutation in different tissues: implications for diagnosis." Am J Med Genet A 130A(2): 134-137.

Sofou, K., K. Steneryd, L. M. Wiklund, M. Tulinius and N. Darin (2013). "MRI of the brain in childhood-onset mitochondrial disorders with central nervous system involvement." Mitochondrion 13(4): 364-371.

Sokoloff, L., M. Reivich, C. Kennedy, M. H. Des Rosiers, C. S. Patlak, K. D. Pettigrew, ... M. Shinohara (1977). "The [14C]deoxyglucose method for the measurement of local cerebral glucose utilization: theory, procedure, and normal values in the conscious and anesthetized albino rat." $\underline{J}$ Neurochem 28(5): 897-916.

Sparaco, M., A. Simonati, T. Cavallaro, L. Bartolomei, M. Grauso, F. Piscioli, . . . N. Rizzuto (2003). "MELAS: clinical phenotype and morphological brain abnormalities." Acta Neuropathol 106(3): 202-212.

Stuber, C., M. Morawski, A. Schafer, C. Labadie, M. Wahnert, C. Leuze, .. R. Turner (2014). “Myelin and iron concentration in the human brain: a quantitative study of MRI contrast." Neuroimage 93 Pt 1: 95-106.

Syka, J. (2002). "Plastic changes in the central auditory system after hearing loss, restoration of function, and during learning." Physiol Rev 82(3): 601-636.

Teeuwisse, W. M., W. M. Brink and A. G. Webb (2012). "Quantitative assessment of the effects of high-permittivity pads in 7 Tesla MRI of the brain." Magn Reson Med 67(5): 1285-1293.

Tomasi, D., G. J. Wang and N. D. Volkow (2013). "Energetic cost of brain functional connectivity." Proc Natl Acad Sci U S A 110(33): 13642-13647.

Trattnig, S., E. Springer, W. Bogner, G. Hangel, B. Strasser, B. Dymerska, .. S. D. Robinson (2016). "Key clinical benefits of neuroimaging at 7T." Neuroimage.

Tschampa, H. J., H. Urbach, S. Greschus, W. S. Kunz and C. Kornblum (2013). “Neuroimaging characteristics in mitochondrial encephalopathies associated with the m.3243A>G MTTL1 mutation." J Neurol 260(4): 1071-1080.

Tsujikawa, K., J. Senda, K. Yasui, Y. Hasegawa, M. Hoshiyama, M. Katsuno and G. Sobue (2016). "Distinctive distribution of brain volume reductions in MELAS and mitochondrial DNA A3243G mutation carriers: A voxel-based morphometric study." Mitochondrion 30: 229-235.

Tsujikawa, T., M. Yoneda, Y. Shimizu, H. Uematsu, M. Toyooka, M. Ikawa, . . H. Kimura (2010). "Pathophysiologic evaluation of MELAS strokes by serially quantified MRS and CASL perfusion images." Brain Dev 32(2): 143-149.

Ugurbil, K. (2017). "Imaging at ultrahigh magnetic fields: History, challenges, and solutions." Neuroimage.

Uludag, K. and P. Blinder (2017). “Linking brain vascular physiology to hemodynamic response in ultra-high field MRI." Neuroimage.

Van der Elst, W., M. P. van Boxtel, G. J. van Breukelen and J. Jolles (2005). "Rey's verbal learning test: normative data for 1855 healthy participants aged 24-81 years and the influence of age, sex, education, and mode of presentation." J Int Neuropsychol Soc 11(3): 290-302.

van der Elst, W., M. P. van Boxtel, G. J. van Breukelen and J. Jolles (2006). "The Letter Digit Substitution Test: normative data for 1,858 healthy participants aged 24-81 from the Maastricht Aging Study (MAAS): influence of age, education, and sex." J Clin Exp Neuropsychol 28(6): 998-1009.

Van der Elst, W., M. P. Van Boxtel, G. J. Van Breukelen and J. Jolles (2006). “The Stroop color-word 
test: influence of age, sex, and education; and normative data for a large sample across the adult age range." Assessment 13(1): 62-79.

van der Knaap, M. S., I. Boor and R. Estevez (2012). “Megalencephalic leukoencephalopathy with subcortical cysts: chronic white matter oedema due to a defect in brain ion and water homoeostasis." Lancet Neurol 11(11): 973-985.

van der Zwaag, W., A. Schafer, J. P. Marques, R. Turner and R. Trampel (2016). “Recent applications of UHF-MRI in the study of human brain function and structure: a review." NMR Biomed 29(9): 1274-1288.

Vaughan, J. T., M. Garwood, C. M. Collins, W. Liu, L. DelaBarre, G. Adriany, ... K. Ugurbil (2001). “7T vs. 4T: RF power, homogeneity, and signal-to-noise comparison in head images." Magn Reson Med 46(1): 24-30.

Virtanen, S. M., M. M. Lindroos, K. Majamaa, P. Nuutila, R. J. Borra and R. Parkkola (2011).“'Voxelwise analysis of diffusion tensor imaging and structural MR imaging in patients with the m.3243A $>G$ mutation in mitochondrial DNA." AJNR Am J Neuroradiol 32(3): 522-526.

Whittaker, R. G., J. K. Blackwood, C. L. Alston, E. L. Blakely, J. L. Elson, R. McFarland, ... R. W. Taylor (2009). "Urine heteroplasmy is the best predictor of clinical outcome in the m.3243A>G mtDNA mutation." Neurology 72(6): 568-569.

Yacoub, E., N. Harel and K. Ugurbil (2008). "High-field fMRI unveils orientation columns in humans." Proc Natl Acad Sci U SA 105(30): 10607-10612.

Yushkevich, P. A., J. Piven, H. C. Hazlett, R. G. Smith, S. Ho, J. C. Gee and G. Gerig (2006). “User-guided 3D active contour segmentation of anatomical structures: significantly improved efficiency and reliability." Neuroimage 31(3): 1116-1128.

Zhu, X. H., H. Qiao, F. Du, Q. Xiong, X. Liu, X. Zhang, ... W. Chen (2012). “Quantitative imaging of energy expenditure in human brain." Neuroimage 60(4): 2107-2117. 


\section{CHAPTER 4}

\section{6. | SUPPLEMENTARY MATERIALS}
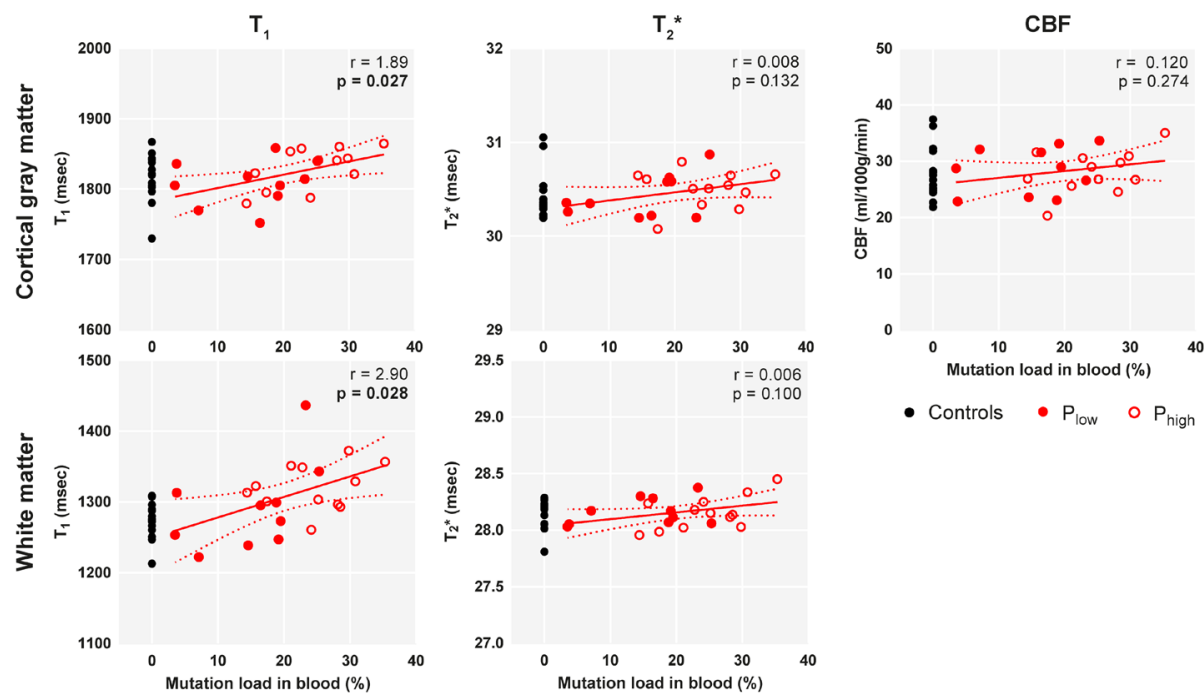

Fig. S4.1. | Scatterplots showing the correlation between global cGM (top) and WM (bottom) $T_{1} T_{2}^{*}$ or CBF and mutation load in blood within m.3243A>G patients, adjusted for age and gender. Mean values for each control subject (black dots) are displayed for reference and red solid lines represent the best fit $\pm 95 \% \mathrm{Cls}$. Boldface $p$-values indicate a significant correlation between mutation load in blood and cGM thickness, $T_{1}, T_{2}{ }^{*}$ or CBF after correction for age and gender. 
A
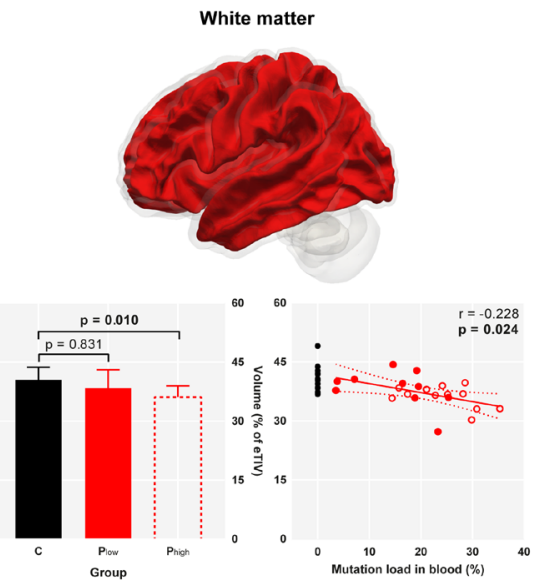

C Between groups WM Mutation load vs. WM $T_{1}$, or $T_{2}^{*}$ $T_{1}$ or $T_{2}^{*}$

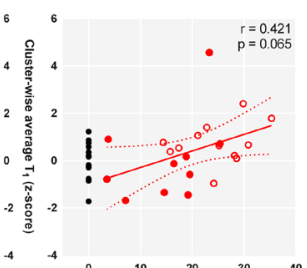

$$
\text { Controls Pow Phigh }
$$
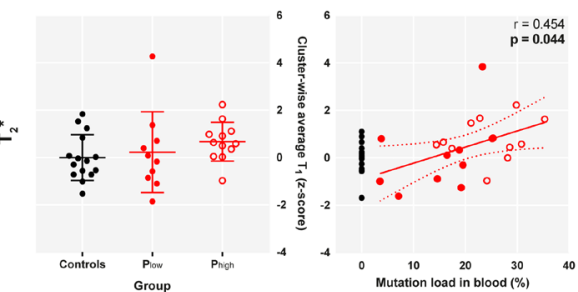

B
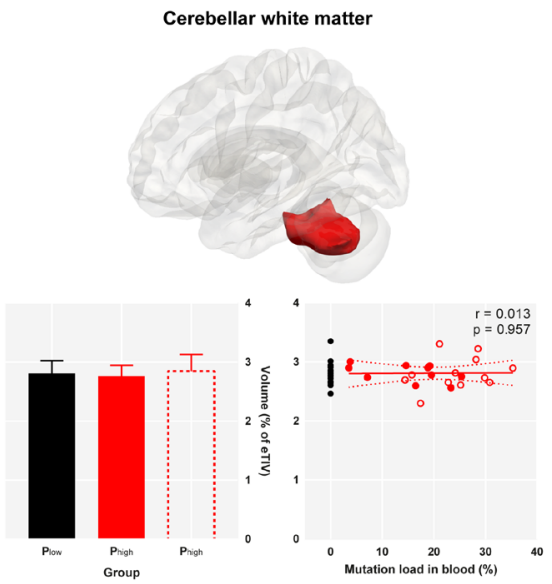

D
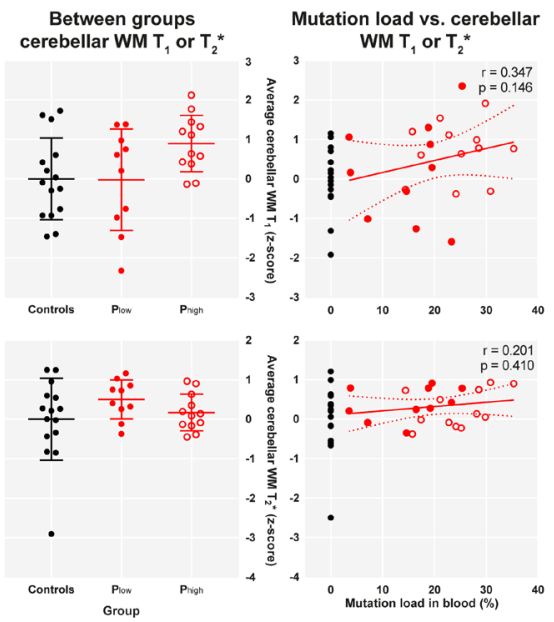

- Controls $\bullet \mathrm{P}_{\text {low }} \quad \ldots . . . . . \mathrm{P}_{\text {high }}$

Fig. S4.2. | Between and within group comparison of total WM (A) and cerebellar WM volumes (B), $T_{1}(C)$ and $T_{2}^{*}(D)$. Bar plots display unadjusted mean ( \pm S.D.) volumes for each structure for controls (black) and $P_{\text {low }}$ (filled red) and $P_{\text {high }}$ (dashed red bars) mutation load in blood. Dot plots display unadjusted mean ( \pm S.D.) $T_{1}$ or $T_{2}{ }^{*}$ values for controls (black), $P_{\text {low }}$ (filled red) and $P_{\text {high }}$ (empty red dots). Scatterplots show the correlation between volume, $T_{1}$ or $T_{2}{ }^{*}$ and mutation load in blood for $m .3243 A>G$ patients, adjusted for age and gender. Mean values for each control subject (black dots) are displayed for reference and red solid lines represent the best fit $\pm 95 \% \mathrm{Cl}$. Boldface $p$-values indicate a significant correlation between mutation load in blood and volume, $T_{1}$ or $T_{2}{ }^{*}$ after correcting for age and gender. 


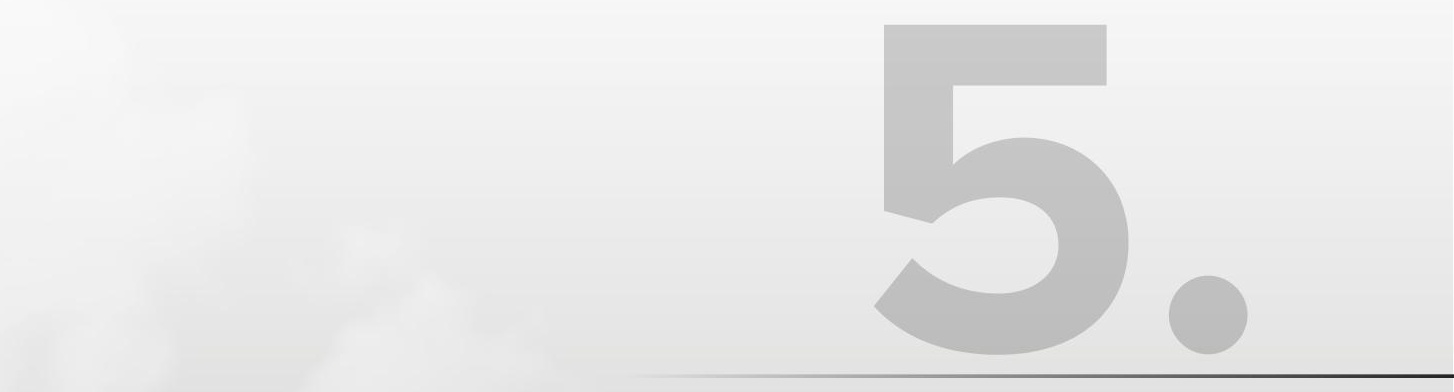

IMAGING GENETICS IN TYPE 2 DIABETES

ROY A.M. HAAST

MICHIEL E. ADRIAENS

CARLA J. VAN DER KALLEN

COEN D.A. STEHOUWER

MIRANDA T. SCHRAM

MARTIN VAN BOXTEL

HUBERT J.M. SMEETS

JACOBUS F.A. JANSEN

ELIA FORMISANO

\& KÂMIL ULUDAĞ 


\section{CHAPTER 5}

\section{ABSTRACT}

Type 2 Diabetes Mellitus (T2DM) is a chronic metabolic disease that gradually develops through progressive impairment of glucose metabolism. It is induced through a combination of environmental and genetic risk factors. Elevated fasting glucose levels, hypertension, obesity and/or dyslipidemia - also known as the 'metabolic syndrome (MetS)' - are commonly observed phenotypical risk factors in subjects at risk to develop T2DM. However, the complex genetic nature remains largely unknown. Eventually, T2DM may lead to damage of many organs, including the cardiovascular and/or central nervous systems. In the current study, we focused on the genetical and neuroradiological differences in subjects affected by T2DM $(\mathrm{N}=44)$ and MetS $(\mathrm{N}=44)$, compared to controls ( $\mathrm{N}=45)$, using whole-exome sequencing and 7T MRI, respectively. We describe the different protocols, as well as the typical processing workflows that were used to obtain and analyze the individual datasets, respectively, and show preliminary output, such as the groups' demographics, example FreeSurfer morphological data and metabolomic profiles. Finally, as the full analysis is ongoing, we conclude with potential analyses strategies to identify biomarkers for T2DM-related neurodegeneration and/or cognitive impairment, based either on uni-modal, but also combined analyses of the multi-modal datasets. 


\section{1. | INTRODUCTION}

Traditionally, single cortical measurements, such as thickness or volume, are linked to functional outcome measures, e.g. cognitive performance (for example, see previous chapter in this thesis). However, an approach that combines information from multi-parameter (quantitative) MRI and other phenotypical, including genetic and metabolomic data, followed by multivariate data analysis may be more promising in finding biomarkers. This 'imaging genetics' approach is emerging in neuroscience research, and aims at associating neuroimaging observations with a specific set of genetic variants and/or metabolic profile. Hence, in the current chapter, we pursued such an approach in a clinical population affected by Type 2 Diabetes Mellitus (T2DM). T2DM is a metabolic disease characterized by high global prevalence. The total number of people with T2DM was estimated at 171 million individuals world-wide in 2000 and is projected to rise to 366 million in 2030 (Wild et al., 2004). Hereafter, we will first briefly review the pathogenesis of T2DM, focusing hereby on the observed changes in glucose metabolism, brain and cognitive performance, as well as the most significant genetical and metabolomic findings. Second, we define the rationale and protocol of the study, including a description of the different data modalities that were acquired. Finally, as analysis of the data is work-in progress, we show the study population demographics, illustrate some preliminary results and discuss potential follow up analyses.

\subsection{1. | Type 2 Diabetes Mellitus}

T2DM is a common chronic metabolic disorder characterized by high blood glucose (i.e. hyperglycemia) induced by increasing insulin resistance and relative insulin deficiency that develops throughout the years (Fu et al., 2013). It induces complex and multifactorial metabolic changes, which very often lead to damage and impairment of many organs, including the brain and the cardiovascular system (e.g. diabetes was estimated to account for $11 \%$ of vascular deaths, Sarwar et al. (2010)). As such, it can lead to substantially increased morbidity and mortality if not treated properly (Rao Kondapally Seshasai et al., 2011).

The progression of normal glucose metabolism (fasting glucose levels $<6.1 \mathrm{mmol} / \mathrm{L}$ ) to T2DM ( $\geq 7.0 \mathrm{mmol} / \mathrm{L}$ ) is a gradual process, in which insulin resistance plays a crucial role (Weir and Bonner-Weir, 2004; Fu et al., 2013). Insulin resistance, before the clinical presentation with T2DM, is often accompanied by other metabolic and vascular abnormalities. The cluster of these cardiovascular risk factors (abdominal obesity, hypertension, dyslipidemia and impaired fasting glucose (IFG), see also Fig. 5.1), referred to as the 'metabolic syndrome' (MetS), is often considered as a pre-diabetic condition 


\section{CHAPTER 5}

(Lorenzo et al., 2003). In the US, approximately 34\% and 35\% of the men and women older than 20 years have MetS, respectively (Cameron et al., 2004). However, IFG by itself, or impaired glucose tolerance (IGT), without complying to the diagnostic criteria of T2DM, are considered pre-diabetic stages as well. IFG and IGT are characterized by high fasting (6.1 - $6.9 \mathrm{mmol} / \mathrm{L})$ or post-load glucose levels $(7.8-11.1 \mathrm{mmol} / \mathrm{L})$, respectively, and present in $16.1 \%$ of the US population (Li et al., 2009). Pre-diabetic (IFG and IGT) individuals or those complying with the MetS conditions have an increased risk to develop T2DM, but are also characterized by an increased cardiovascular disease risk (Gami et al., 2007; Ford et al., 2010).

\subsection{2. | Neuropathological observations}

Abnormalities of the brain associated with T2DM that are visible at MRI (i.e. macro-scale) include atrophy, white matter lesions, silent lacunar infarcts and microbleeds (Brundel et al., 2014; van Bussel et al., 2017). Macrostructural imaging using conventional 1.5T and 3T MRI showed accelerated loss of brain volume due to T2DM, compared to normal aging (de Bresser et al., 2010; Falvey et al., 2013). Here, neurodegenerative symptoms as indicated by loss in cortical thickness and/or volume - were especially observed in the cortical and subcortical medial temporal structures (including hippocampus) and frontal lobe (Brundel et al., 2010; Moran et al., 2013). Other neuropathological features visible using structural MRI, such as local $\mathrm{T}_{1} \mathrm{~W}$ and $\mathrm{T}_{2}{ }^{(*)} \mathrm{W}$ signal hyper- and/or hypointensities, are also frequently observed in T2DM brains and are characteristic for the pathological changes related to cerebral small vessel disease, including white matter lesions (WMLs, i.e. hyperintense on $\mathrm{T}_{2} \mathrm{w}$ images), silent brain infarcts (SBIs, i.e. hypointense on $\mathrm{T}_{1} \mathrm{w}$ images) and lacunar abnormalities (i.e. mostly identified using a combination of contrasts) (see for example the work presented in Jongen et al. (2007), Umemura et al. (2011) and Moran et al. (2013)). In addition, it is hypothesized that T2DM is associated with an impaired pattern of the so-called default-mode network function, which may be involved in the neuropathology of the disease (Musen et al., 2012), as well has significant consequences for cognition (van Elderen et al., 2010; Biessels et al., 2014). Quantitative measures of brain networks functioning can be obtained using functional MRI (fMRI), without introducing external stimuli, but by keeping the subject in a resting-state (rs-fMRI) (Biswal et al., 1995; Bullmore and Sporns, 2009). For example, van Bussel et al. (2016) demonstrated that T2DM subjects harbor altered functional brain networks, which possibly explain the reduced cognitive processing speed in these subjects. Note that in T2DM as well as in other diseases, decreases or increases in apparent connectivity obtained via rs-fMRI may be confounded by altered baseline cerebral blood flow (CBF) and/or vascular reactivity. 
However, fine-scale changes that precede the macrostructural and/or functional manifestations and the impaired cerebral hemodynamics discussed above may remain unnoticed using conventional $1.5 \mathrm{~T}$ or $3 \mathrm{~T} \mathrm{MRI}$ and with the typical MRI sequences that are used in the clinic (Conijn et al., 2011; Theysohn et al., 2011). Therefore, more advanced MRI is necessary to identify these changes, for example, by moving to ultra-high field (UHF) strengths and/or quantitative acquisition methods. Such techniques can potentially unravel and quantify fine-scale changes, including (sub)cortical gray and white matter microstructural changes in pre-diabetic subjects, leading to the frequently observed macrostructural abnormalities reviewed above in T2DM. In addition, CBF measurements can provide insights into the baseline and functionally-induced cerebral metabolism. Here, some studies report lower cerebral perfusion in T2DM and suggest that this may provide an early indication of altered cerebral vasoreactivity and pathological levels of oxygen and glucose metabolism (Last et al., 2007; Jansen et al., 2016).

\subsection{3. | Changes in cognitive performance}

Cognitive impairment in MetS and T2DM, resulting from the brain phenotypical changes described above, has been widely studied. An increasing amount of studies reported subtle changes (i.e. decreases) in cognitive functioning in these subjects compared to controls. According to reviews by Reijmer et al. (2010) and more recently Biessels et al. (2014), not only memory, but also other cognitive domains become impaired. Attention, executive functioning and information processing speed, show consistency among different studies, with lower values observed for T2DM subjects. For example, T2DM subjects older than 40 years showed significant decreases in information-processing speed and executive functioning after a 12 years follow-up compared to control, non-diabetic, subjects. In addition, they performed significantly worse on a memory-related task (Spauwen et al., 2013). Comparable findings have been reported in pre-diabetic (i.e. IGT or MetS) subjects (Lamport et al., 2009; Crichton et al., 2012). In addition, (pre-)diabetic subjects have also an increased risk to develop more severe types of impaired cognitive functioning. For example, compared to subjects without diabetes, diabetics have a $50 \%$ greater risk of developing dementia (Biessels et al., 2006; Cheng et al., 2012).

\subsection{4. | Genetic susceptibility for T2DM}

Development of T2DM results from the interaction of environmental factors with a set of genetic variants (Temelkova-Kurktschiev and Stefanov, 2012). The most influential environmental factors include excessive caloric intake (e.g. sedentary lifestyle, obesity 


\section{CHAPTER 5}

etc.) and lack of exercise or expenditure of this energy. The effects of these environmental factors on T2DM risk are well-described by epidemiological studies and can be used as targets for lifestyle interventions to slow down T2DM development and minimize subsequent changes of the brain's structure and functioning (Johnson et al., 2012). However, the genetic base of this interaction remains elusive and non-trivial to counteract. Unlike, for example, 'mitochondrial diabetes', which can be initiated via one specific genetic mutation (i.e. m.3243A $>\mathrm{G}$, see Chapter 4 in this thesis) (Maassen et al., 2004), the genetics of T2DM are complex, diverse and not easily unraveled. It has been proposed that T2DM is induced through interplay of numerous genetic variants (Morris et al., 2012). Hereby, a positive family history leads to a 2- to 4-fold increased risk for T2DM (Stumvoll et al., 2005).

Genetic variants can be detected using sequencing techniques. The field of genetic sequencing evolved rapidly during the last couple of decades and has led to the first version of the entire human genome (Venter et al., 2001). While sequencing of the human genome was estimated to have costs $\$ 2.7$ billion using first-generation sequencing, nowadays, whole-genome sequencing is possible for much lower costs (down to \$1000) using sophisticated techniques like next-generation sequencing (NGS) (Hayden, 2014; Goodwin et al., 2016). As such, this greatly promoted the use of genomic profiling in a wide-range of applications and advanced the understanding of diseases at the genomic level. Based on the study design and depending on the number of subjects, the coverage (and depth) can be adjusted to range from the whole-genome (introns and exons) to whole-exome (all exons, this study), specific exomes (selected exons) or gene panels (selected genes).

Most findings in the field of T2DM and genetics rely on whole-genome association studies (GWAS) and familial inheritance studies (Florez et al., 2003). GWAS studies are aimed at discovering common genetic variants that are highly associated with a specific trait or disease. Most often, these genetics variants encompass only very small and subtle (i.e. one base pair) differences, also known as single-nucleotide polymorphisms (SNPs). SNPs are very common and therefore the difficulty lies in finding those that are significantly (e.g. $p<0.05 \times 10^{-8}$ in typical GWAS studies) associated (individually or as a cluster) with an increased onset of T2DM. This p-value is so low because of the multiple comparisons that are necessary due to the high degree of different SNPs in each subject (Pe'er et al., 2008). As such, the DIAbetes Genetics Replication And Meta-analysis (DIAGRAM) consortium combined several large-scale studies to characterize the genetic basis of T2DM (Morris et al., 2012; Mahajan et al., 2014). Their population database includes 34,840 cases and 114,981 controls. Several genetic loci are 
suspected to pose a risk for T2DM. The TCF7L2 gene is most probably associated with T2DM and can be observed across a wide range of ethnicities. A recent meta-analysis combined the results from different T2DM GWAS studies consortia and revealed additional significant associated SNPs with T2DM, such as on the TMEM154, SSR1-RREB1 and FAF1 loci. Familial inheritance and twin studies furthermore highlighted several other susceptibility genes for T2DM, including PPARy, ABCC8 and KCNJ11 (Barroso et al., 2003). In T2DM, the absolute risk to siblings of someone with T2DM is $30 \%-40 \%$, as compared to a population prevalence of $7 \%$, providing an increased relative risk for siblings of four to six fold (Florez et al., 2003).

\subsection{5. | Metabolic changes}

Metabolomic phenotyping using mass or nuclear magnetic resonance (NMR) spectroscopy can provide insights into the metabolic changes occurring in subjects developing T2DM (Roberts et al., 2014). The measured metabolome reflects the end-product of the genome, modified through systemic and environmental influences (i.e. 'exposome') on the integrity of genes, enzymes, and related proteins in complex ways. It includes all metabolites in a tissue of interest needed for growth, maintenance, or normal functioning in a specific physiological state and can be used as metabolic signatures. As such, metabolomics provides useful information about the metabolic changes in MetS and/orT2DM. Most of the available studies that used metabolomics in MetS and/or T2DM populations focused on lipid and amino acid metabolism. Besides glucose, alterations in branched-chain amino acids' (BCAAs), or derivates' (especially 3-methyl-2-oxovalerate) levels seem to be most characteristic for insulin resistance and pre-diabetes. However, also other metabolites were present in different quantities across these groups, including for example glyoxylate, hexosamine and glucosamine (Padberg et al., 2014; Roberts et al., 2014; Abu Bakar et al., 2015).

\section{2. | RATIONALE OF THE STUDY}

Understanding the development of T2DM, as well as related pathological features (e.g. neurodegeneration and/or decreases in cognitive performance) is a complex issue. As such, studies will benefit from an approach that combines information from multiple data modalities measured across non-diabetic, MetS with IFG (i.e. pre-diabetic) and diabetic subjects. Using advanced statistical analysis methods for combined interferences across multiple parameters (Liu and Calhoun, 2014), we aim to identify neuroimaging biomarkers using 7T MRI that: aim 1) change gradually between groups that differ in their metabolic and cardiovascular health status (i.e. from controls to MetS to T2DM subjects), and aim 2) correlate with individual cognitive tests performance. 


\section{CHAPTER 5}

Instead of using conventional 1.5T or 3T MRI, as in most of the earlier T2DM studies, utilization of UHF 7T MRI will further enhance the probability of finding MR biomarkers. Data acquisition at 7T is expected to improve data quality for examining structural and functional connectivity compared to scanning at 1.5T or 3T due to improvements in contrast-to-noise (CNR) and signal-to-noise ratios (SNR) (Pohmann et al., 2016). As such, scanning at 7T enables high spatial resolution quantitative mapping of $\mathrm{T}_{1^{\prime}}$ $\mathrm{T}_{2}{ }^{*}$ and magnetic susceptibility $(\mathrm{X})$ variations, that allow to directly compare across subjects and groups. In addition to methods, such as ASL and fMRI, mapping (i.e. voxel-wise determination) of absolute $T_{1}, T_{2}{ }^{*}$ and $X$ values also provides helpful information with regards to brain abnormalities. For example, $T_{1}, T_{2}^{*}$ and $x$ high-resolution maps provide information with regards to differences in myelin and iron content up to the micro-structural level (Stuber et al., 2014).

In addition, we will aim 3) explore the effects of possible genetic and metabolomic variations on the brain's structure and functioning (including possible differences in cognition). To accomplish this, we will perform whole-exome sequencing in the same subjects that will undergo MR imaging and for which metabolomics data is available. While our sample size is rather large for an UHF neuroimaging study, GWAS analysis approaches typically require many more subjects. Therefore, we will apply a hypothesis-driven approach and focus on genes/pathways that: a) have previously been shown to vary in T2DM and/or MetS (Mahajan et al., 2014), b) are related to cognitive functioning (Cox et al., 2014) or c) are related to MRI parameters (Khadka et al., 2016). For the selection of genes, we will consider only effects that have been reported as highly significantly $\left(p<0.05 \times 10^{-8}\right)$ correlated with these factors in earlier GWAS studies.

Until now, no study has been published yet combining imaging with genetics (and metabolomics) in T2DM. Our results may help future research to characterize patient groups that are at risk to develop T2DM, neurodegeneration and/or cognitive impairment based on their genetic, metabolic and MRI profile. Furthermore, although our sample size only allows testing a limited set of genes, the availability of whole-exome data allows integrating the present study with other future studies of imaging/ genetics.

\section{3. | STUDY PROTOCOL}

\subsection{1. | Subject selection procedure}

A total of 133 participants were recruited based on a specific selection procedure from the eligible participants $(\mathrm{N}=620)$ within the total subject pool of The Maastricht Study 

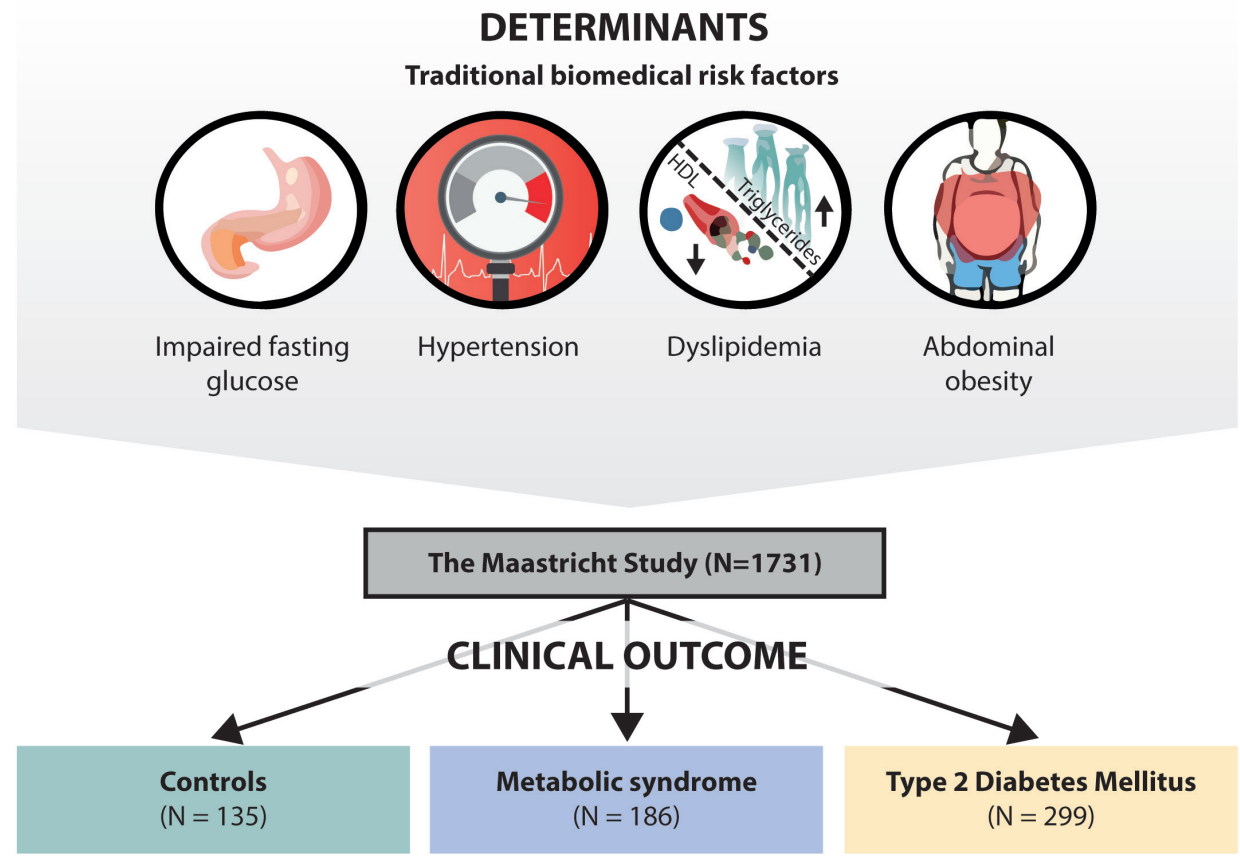

Study-specific group characteristics:

$\begin{array}{lll}\text { - Two MetS criteria } & \text { - Three or more MetS criteria } & \text { - Two or more MetS criteria } \\ \text { - Normal fasting glucose levels } & \text { - Elevated fasting glucose levels } & \text { - Type } 2 \text { Diabetes Mellitus } \\ & \text { (i.e. } 100-125 \mathrm{mg} / \mathrm{dL} \text { ) } & \text { - No insulin use } \\ & \text { - No Type } 2 \text { Diabetes Mellitus } & \end{array}$

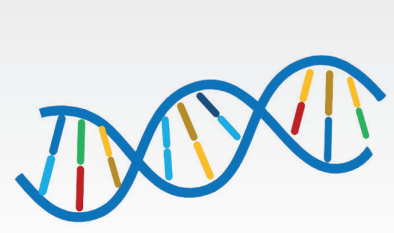

Genomics

\section{Advanced phenotyping}

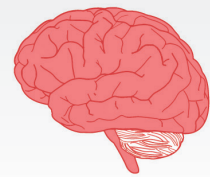

Ultra-high field MRI

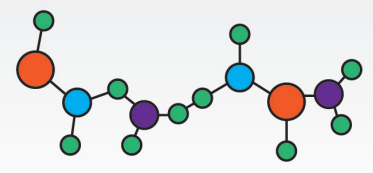

Metabolomics

Fig. 5.1. An advanced phenotyping approach is applied to a subset of The Maastricht Study. The top of the figure shows main categories of determinants that are measured. The middle of the figure presents the main clinical outcomes that are studied, while the bottom of the figure shows the methods used for the advanced phenotyping of the subgroups.

( $\mathrm{N}=1731$ with cleaned data) (Schram et al., 2014). The Maastricht Study is an ongoing observational and prospective cohort study that investigates the etiology, pathophysiology, complications and comorbidities of T2DM, focusing on the population in the southern part of Limburg, in the Netherlands. The rationale, overall study design, recruitment strategy and methods of basic measurements, and an overview of all measurements can be found in the original paper. For the current study, participants were 


\section{CHAPTER 5}

selected based on the available data using the following criteria: (i) completed MRI session at 3T with no incidental findings reported, (ii) metabolomics data available and (iii) completed neuropsychological examination.

Secondly, from the eligible participants, subjects (aged between 40 and 75 years) were selected from one of three groups: controls $(N=135)$, MetS $(N=186)$ or T2DM $(N=299)$. Here, each of the control subjects was characterized by exactly two of the five MetS criteria (see top part of Fig. 5.1, where dyslipidemia refers to either i) increased triglycerides or ii) low HDL cholesterol levels), however, without elevated fasting glucose levels (i.e. $<6.0 \mathrm{mmol} / \mathrm{L}$ ). In contrast, participants were only eligible for inclusion within the MetS group in case of three or more out of the five MetS criteria, including at least increased fasting glucose levels (i.e. 6.0-6.9 mmol/L), but no T2DM. Here, T2DM was defined following the WHO 2016 criteria and was true in case the participant was using diabetes medication, or if they had a fasting blood glucose level $\geq 7.0 \mathrm{mmol} / \mathrm{L}$ and/ or a 2 -h blood glucose $\geq 11.1 \mathrm{mmol} / \mathrm{L}$ after an oral glucose tolerance test (OGTT). In addition, all participants within the T2DM group were diagnosed with T2DM and a minimum of two out of five MetS criteria. Finally, participants were excluded from this group in case of insulin use. See also Fig. 5.1 (middle part) for the group-specific in- and exclusion criteria. Eventually, subjects were contacted for participation. During recruitment, the number of participants per group, as well as the male/female and age distributions were taken into account to closely match the groups. A total of 133 subjects were included, of which 45 controls, 44 MetS and 44 T2DM subjects. All subjects gave written informed consent in accordance with the Declaration of Helsinki. The protocol was approved by the ethics review board of the MUMC+ in Maastricht, The Netherlands. For each subject, we obtained 7T MRI, genetic (using whole-exome sequencing, WES) and metabolomic data. In the next paragraphs, we will explain in more detail how these have been acquired, except for the metabolomics data, which was already available in processed form following the procedure described in Soininen et al. (2015).

\subsection{2. | 7T MRI acquisition protocol}

For all subjects, multi-parameter MR data were acquired using a whole-body 7T magnet (Siemens Medical Systems, Erlangen, Germany), a 32-channel phased-array head coil (Nova Medical, Wilmington, USA) and the sequences described in Table 5.1. Dielectric pads containing a $25 \%$ suspension of barium titanate in deuterated water were placed proximal to the temporal lobe area to locally increase the transmit $\mathrm{B}_{1}{ }^{+}$ field and to improve its homogeneity across the brain (Teeuwisse et al., 2012). See Fig. 5.2 for a schematic overview of the imaging protocol and example data from a single subject. 


\section{Quantitative anatomical and perfusion imaging}

High resolution $\left(0.7 \mathrm{~mm}\right.$ isotropic nominal voxel size) whole-brain quantitative $T_{1}$ (and $\mathrm{B}_{1}{ }^{+}$map), $\mathrm{T}_{2}{ }^{*}$ and QSM (see Fig. 5.2c) maps were obtained for anatomical imaging using the MP2RAGE (Marques et al., 2010), Sa2RAGE (Eggenschwiler et al., 2012) and a ME-GRE sequence, respectively. All anatomical maps were acquired within 20 minutes and with a spatial resolution of $0.7 \mathrm{~mm}$ isotropic nominal voxel size. In addition to the anatomical scans, baseline partial-brain (excluding cerebellum and lower temporal lobes) perfusion was quantified using FAIR QUIPSS II ASL with a 2D simultaneously multi-slice (SMS) echo-planar imaging (EPI) readout and $2.8 \mathrm{~mm}$ isotropic nominal voxel size (Ivanov et al., 2017). One blood equilibrium magnetization $\left(M_{0}\right)$ image was acquired in order to determine absolute CBF values using the same settings but with no ASL preparation pulses and TR of $20 \mathrm{~s}$. The eye centers were taken as reference for the magnet isocenter position in order to improve the ASL labeling efficiency. A standard dual-echo field map was acquired to correct the ASL data post-hoc for EPI readout-related geometrical distortions. See previous chapters of this thesis for more details on the $\mathrm{T}_{1}, \mathrm{~T}_{2}{ }^{*}$ (Chapters 2 and 3 ) and CBF (Chapter 4) mapping protocols.

\section{Functional connectivity mapping}

Finally, two functional MR datasets were acquired. Here, participants performed a fixation task (i.e. resting-state $\mathrm{fMRI}$ ) during the first run, while during the second run, the participants were asked to watch a short movie (i.e. episode of 'How Do They Do It?' on espresso machines), with no sound (except for scanner noise) information (i.e. natural viewing paradigm). Areas that are functionally connected (e.g. homologous areas) are activated most during this kind of stimulation (Bartels and Zeki, 2005). For each run, $T_{2}{ }^{*} w$ GRE-EPI were acquired with an isotropic nominal voxel size of $1.4 \mathrm{~mm}$ and TR of $2 \mathrm{sec}$. The full fMRI session comprised 2 runs of 10:20 min and a total of $300 \mathrm{EPI}$ volumes for each run. In between the two fMRI sessions, five EPI volumes were acquired with opposite phase encoding for spatial distortion correction during post-processing.

\subsection{3. | Whole-exome sequencing}

For the current study, whole-exome sequencing (WES) was used to sequence all exomes (i.e. protein-coding regions) for each subject. Typically, NGS involves several steps from the initial preparation of blood samples to obtain a complete and accurate record of the genetic code (see next paragraph). Blood samples collected by The Maastricht Study were used to extract the DNA for each participant and prepared as suggested by the manufacturer (Illumina, San Diego, CA, USA). Briefly, for each subject, the sample preparation included DNA fragmentation, hybridization to the DNA library, 


\section{CHAPTER 5}

A

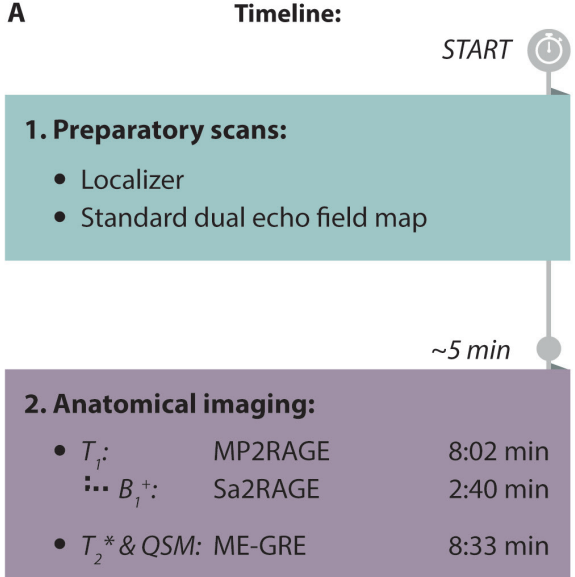

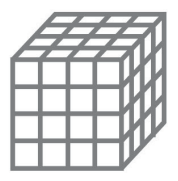

$0.7 \mathrm{~mm}^{3}$
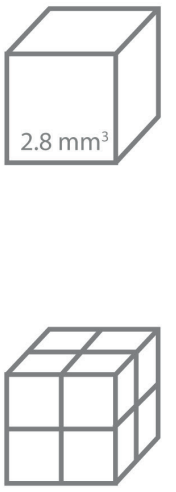

$1.4 \mathrm{~mm}^{3}$
C Example data:
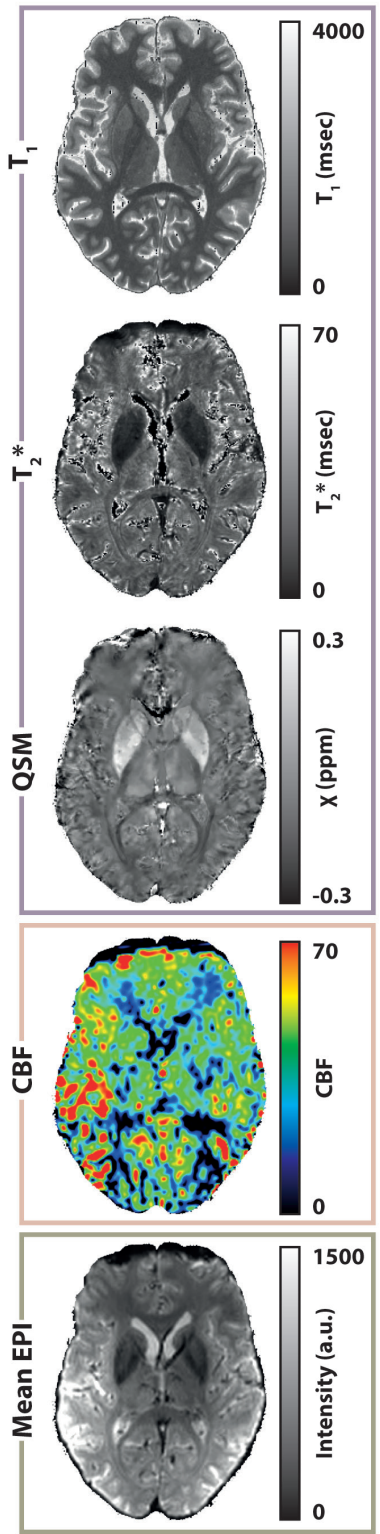

END (total scanning time: $\sim 60 \mathrm{~min}$.)

Fig. 5.2. Schematic depiction of the acquisition protocol. (A) The total protocol lasts for $\sim 60 \mathrm{~min}$. and includes quantitative anatomical and perfusion mapping, as well as two functional runs (resting-state and natural viewing paradigm), (B) Differences in spatial resolution across sequences and (C) example data for a single subject. 


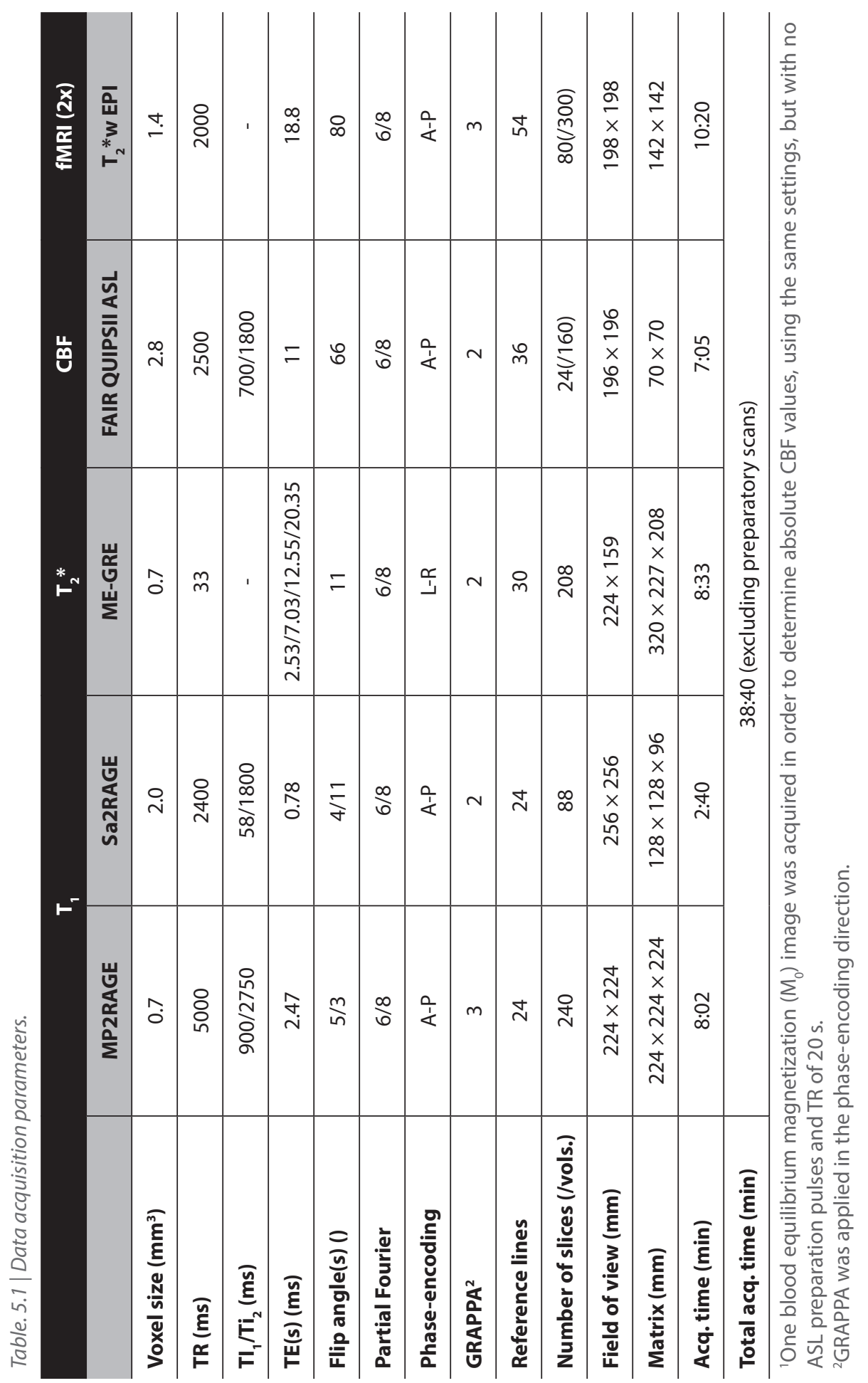




\section{CHAPTER 5}

regions of interest selection (i.e. exomes), a second hybridization step and DNA library enrichment. Finally, the library was quantified and quality-checked, followed by generation of clusters before the paired-end library was sequenced using the Illumina HiSeq2000 for whole-exome sequencing.

\section{4. | STUDY POPULATION AND DATA PROCESSING}

The 7T MRI acquisitions and the WES generate large amounts of data that need to be processed in a systematic and reproducible way. In the next paragraph, we will first describe the study population, for which MRI and WES data has been obtained using the Maastricht Study dataset, followed by an outline of the processing steps that prepares the data for statistical analyses.

\subsection{1. | Study population}

Table 5.2 shows the baseline characteristics for each group obtained from the Maastricht Study dataset. During recruitment, the groups were matched on age and sex. As expected, significant differences were found across groups for several metabolic variables, including fasting blood glucose (FBG, ANOVA $F_{2,130}=110.404, p<0.0001$ ), $\mathrm{HbA}_{1 \mathrm{c}}\left(\mathrm{F}_{2,129}=76.92, \mathrm{p}<0.0001\right)$, triglyceride $\left(\mathrm{F}_{2,130}=6.651, \mathrm{p}=0.002\right)$ and LDL cholesterol levels $\left(F_{2,130}=7.403, p=0.001\right)$.

Table 5.2 | Study population characteristics.

\begin{tabular}{l|c|c|c}
\hline \multicolumn{2}{|c|}{ Controls (N=45) } & MetS (N=44) & T2DM (N=44) \\
\hline Demographic factors & & & \\
\hline Age (years) & $56.51 \pm 6.9$ & $59.27 \pm 5.8$ & $58.86 \pm 6.9$ \\
\hline Sex, male (\%) & 55 & 50 & 54.5 \\
\hline Education (\%) & & & \\
\hline Low & 8.9 & 11.4 & 20.5 \\
\hline Middle & 33.3 & 52.3 & 50.0 \\
\hline High & 57.8 & 36.4 & 29.5 \\
\hline Smoking status (\%) & & & \\
\hline Never & 44.4 & 29.5 & 32.6 \\
\hline Former & 53.3 & 56.8 & 55.8 \\
\hline Current & 2.2 & 13.6 & 11.6 \\
\hline Alcohol consumption (\%) & & & \\
\hline None & 13.3 & 13.6 & 23.3 \\
\hline Low & 60 & 54.5 & 53.5 \\
\hline High & 26.7 & 31.8 & 23.3 \\
\hline
\end{tabular}




\begin{tabular}{|c|c|c|c|}
\hline & Controls $(\mathrm{N}=45)$ & MetS (N=44) & T2DM (N=44) \\
\hline Cardiovascular disease (\%) & 0 & 0 & $7^{+, \neq}$ \\
\hline \multicolumn{4}{|l|}{ MetS-related variables (\%) } \\
\hline Number of MetS criteria & $2 \pm 0$ & $3.5 \pm 0.7$ & $3.3 \pm 1.1$ \\
\hline Elevated fasting glucose levels & 0 & 100 & 100 \\
\hline Hypertension & 86.7 & 84.1 & 81.8 \\
\hline Elevated triglyceride levels & 8.9 & 50 & 36.4 \\
\hline Triglyceride levels (mmol/L) & $1.18 \pm 0.44$ & $1.75 \pm 0.77^{\S}$ & $1.86 \pm 1.38^{\dagger}$ \\
\hline Low HDL cholesterol & 4.4 & 18.2 & 31.8 \\
\hline Total cholesterol (mmol/L) & $5.39 \pm 1.08$ & $5.62 \pm 0.97$ & $4.79 \pm 1.35^{1, \neq}$ \\
\hline $\mathrm{HDL}(\mathrm{mmol} / \mathrm{L})$ & $1.53 \pm 0.35$ & $1.45 \pm 0.36$ & $1.39 \pm 0.48$ \\
\hline $\mathrm{LDL}(\mathrm{mmol} / \mathrm{L})$ & $3.33 \pm 1.08$ & $3.38 \pm 0.80$ & $2.67 \pm 1.04^{t, \neq}$ \\
\hline Large waist circumference & 100 & 100 & 75 \\
\hline Waist circumference $(\mathrm{cm})$ & $101.52 \pm 6.93$ & $105.03 \pm 9.50$ & $100.70 \pm 11.02$ \\
\hline \multicolumn{4}{|l|}{ MetS-related medication (\%) } \\
\hline Blood pressure lowering medication & 28.9 & 45.5 & 61.4 \\
\hline Lipid-lowering medication & 26.7 & 25.0 & 59.1 \\
\hline \multicolumn{4}{|l|}{ Type 2 diabetes-related variables } \\
\hline Duration of diabetes (years) & - & - & $6.89 \pm 6.37^{*}$ \\
\hline Newly diagnosed diabetes (\%)" & - & - & 27.3 \\
\hline FBG (mmol/L) & $5.14 \pm 0.32$ & $5.92 \pm 0.34^{\S}$ & $7.99 \pm 1.55^{t, \neq}$ \\
\hline $\mathrm{HbA}_{1 c}(\%)$ & $5.37 \pm 0.29$ & $5.57 \pm 0.38$ & $6.66 \pm 0.77^{t, \neq}$ \\
\hline $\mathrm{HbA}_{1 \mathrm{c}}(\mathrm{mmol} / \mathrm{mol})$ & $35.18 \pm 3.12$ & $37.36 \pm 4.04$ & $49.16 \pm 8.42^{\dagger, \neq}$ \\
\hline \multicolumn{4}{|l|}{ Type 2 diabetes medication (\%) } \\
\hline None & 100 & 100 & 34.1 \\
\hline Oral medication & 0 & 0 & 65.9 \\
\hline Insulin use & 0 & 0 & 0 \\
\hline \multicolumn{4}{|l|}{ Other clinical variables } \\
\hline $\mathrm{BMI}\left(\mathrm{kg} / \mathrm{m}^{2}\right)$ & $28.65 \pm 2.56$ & $30.20 \pm 3.71$ & $28.93 \pm 3.93$ \\
\hline $\mathrm{SBP}(\mathrm{mmHg})$ & $138 \pm 16$ & $136 \pm 15$ & $134 \pm 15$ \\
\hline $\mathrm{DBP}(\mathrm{mmHg})$ & $83 \pm 10$ & $79 \pm 9$ & $78 \pm 9$ \\
\hline Hematocrit levels (\%) & $0.43 \pm 0.03$ & $0.42 \pm 0.02$ & $0.42 \pm 0.03$ \\
\hline \multicolumn{4}{|l|}{ Cognitive performance } \\
\hline Baseline MMSE total score & $29.29 \pm 0.79$ & $28.98 \pm 0.93$ & $29.05 \pm 1.10$ \\
\hline Memory & $0.10 \pm 1.01$ & $-0.10 \pm 0.93$ & $-0.16 \pm 0.99$ \\
\hline Information processing speed & $0.14 \pm 0.70$ & $0.23 \pm 0.70$ & $-0.13 \pm 0.66^{\ddagger}$ \\
\hline Executive function $\&$ attention & $0.10 \pm 0.83$ & $0.21 \pm 0.70$ & $0.05 \pm 0.76$ \\
\hline Overall cognitive functioning & $0.11 \pm 0.64$ & $0.14 \pm 0.53$ & $-0.08 \pm 0.59$ \\
\hline
\end{tabular}

Data are mean \pm S.D. Abbreviations: HDL, high-density lipoprotein; LDL, low-density lipoprotein; 


\section{CHAPTER 5}

FBG, fasting blood glucose; $\mathrm{HbA}_{1 c^{\prime}}$ glycated hemoglobin; BMI, body mass index; SBP, systolic blood pressure; DBP, diastolic blood pressure; MMSE, Mini-Mental State Examination. ${ }^{+}$indicates $p<0.05$ between T2DM and controls; ${ }^{*}$ indicates $p<0.05$ between T2DM and MetS; ${ }^{{ }^{\prime}}$ indicates $p<$ 0.05 between MetS and controls. ${ }^{*} \mathrm{~N}=18$, based on only the participants with diabetes, excluding those with newly diagnosed diabetes. " As newly revealed by the OGTT.

\subsubsection{Example imaging data \& analysis pipeline}

In order to systemically process the MRI data, a fully-automated pipeline (i.e. as far as possible) is necessary to bring the structural (i.e. $T_{1}, T_{2}^{*}$ and QSM), perfusion (i.e. $\mathrm{CBF}$ ) and functional (i.e. resting state- $\&$ task-fMRI) images into the same surface and/ or volume space to enable cross-subject/group comparisons and multi-parameter analyses of the brain. We setup an optimized processing pipeline for the currently acquired 7T imaging data (see Fig. 5.4 for a schematic overview).

\section{Pre-processing}

Inter-subject alignment can be achieved using a volume- or surface-based approach, depending on the region(s) of interest and types of analyses. However, both methods rely heavily on the segmentation accuracy and will therefore improve with better image contrast between the different anatomical structures (e.g. gray and white matter and cerebrospinal fluid). As such, the acquired MP2RAGE data are corrected for residual $\mathrm{B}_{1}{ }^{+}$inhomogeneities to improve the cortical and subcortical contrast (see also Chapter 2). In addition, non-brain tissue (e.g. arteries and dura mater) and the typical MP2RAGE 'salt and pepper' noise outside of the brain are masked out using CBS tools to further improve the following processing steps (Bazin et al., 2014).

\section{Volume-\& surface-based processing}

While surface-based processing and inter-subject coregistration (as applied throughout this thesis) is especially useful for cortical GM group-wise analyses, complementary volume-based analyses additionally enable investigation of WM and subcortical GM tissue. Therefore, the individual MP2RAGE brains are piped through each of these processing streams, using Freesurfer v6.0 and SPM12, respectively (Ashburner, 2009; Fischl, 2012). However, it is important that both streams process the data at the native resolution (i.e. $0.7 \mathrm{~mm}^{3}$ ) to ensure that the spatial details are preserved. Where necessary (e.g. typically near the inferior frontal and temporal lobes or sagittal sinus, especially for 7T MP2RAGE data), segmentation results are manually corrected (see also Chapter 2). In addition, a multi-modal surface matching algorithm (e.g. based on folding pattern, cortical thickness and $T_{1}$ ) will be applied to optimize the inter-subject surface alignment and minimize the loss of spatial specificity of the 7T data (Robinson et al., 2014). Finally, the reconstructed WM surfaces are used to navigate inter-modal 


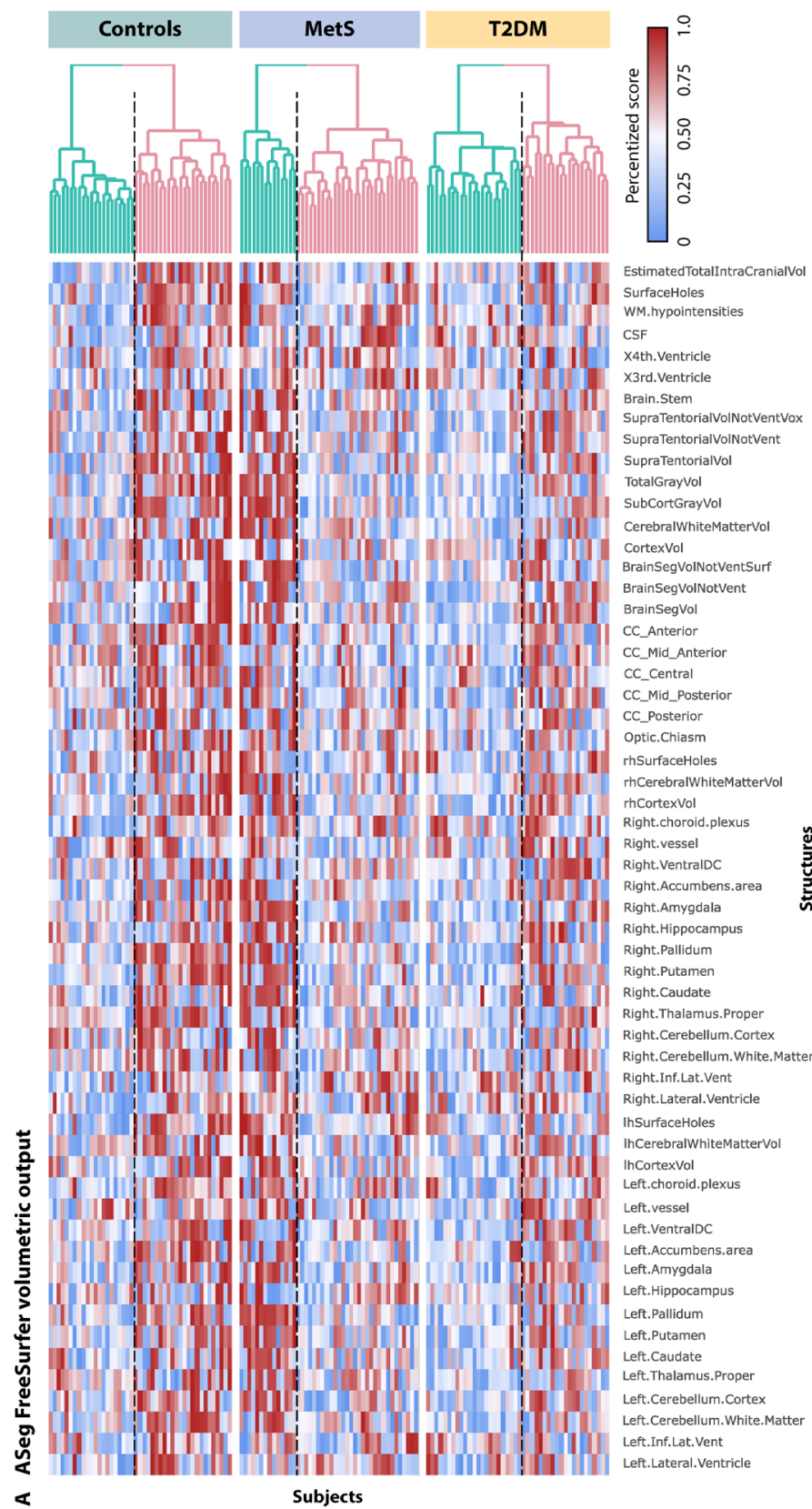

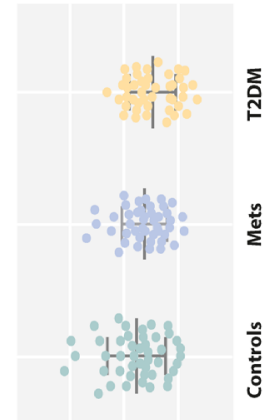
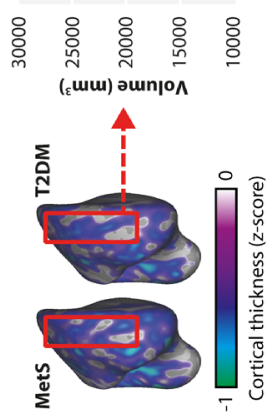

$\dot{s}$

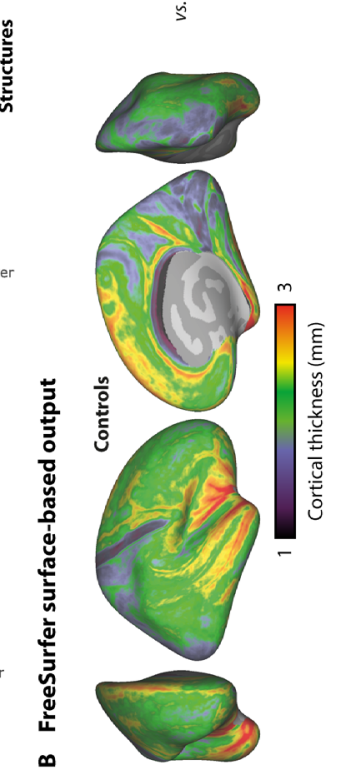

Fig. 5.3. |Example FreeSurfer volume- and surface-based output. Hierarchical clustering of the volumetric (i.e. morphological) normalized data obtained using FreeSurfer may reveal additional sub-phenotypes (A). Moreover, group-average surface maps, such as cortical thickness, can be computed to compare MetS and T2DM subjects with controls (B) to identify regions-of-interest, like the superior frontal gyrus. 


\section{CHAPTER 5}

coregistration and to map the quantitative MRI data into subject's and group surface space for subsequent statistical analyses (for example, see Fig. 5.3B).

For the volume-based pipeline, diffeomorphic coregistration across subjects is performed using the DARTEL algorithm implemented in the Voxel-Based Quantification (VBQ) toolbox for SPM12 (Draganski et al., 2011). VBQ is used to normalize each subject's brain to MNI space for statistical analysis, in a way that the quantitative $T_{1}, T_{2}{ }^{*}$ and CBF values within the particular tissue classes of interest are preserved and, im-

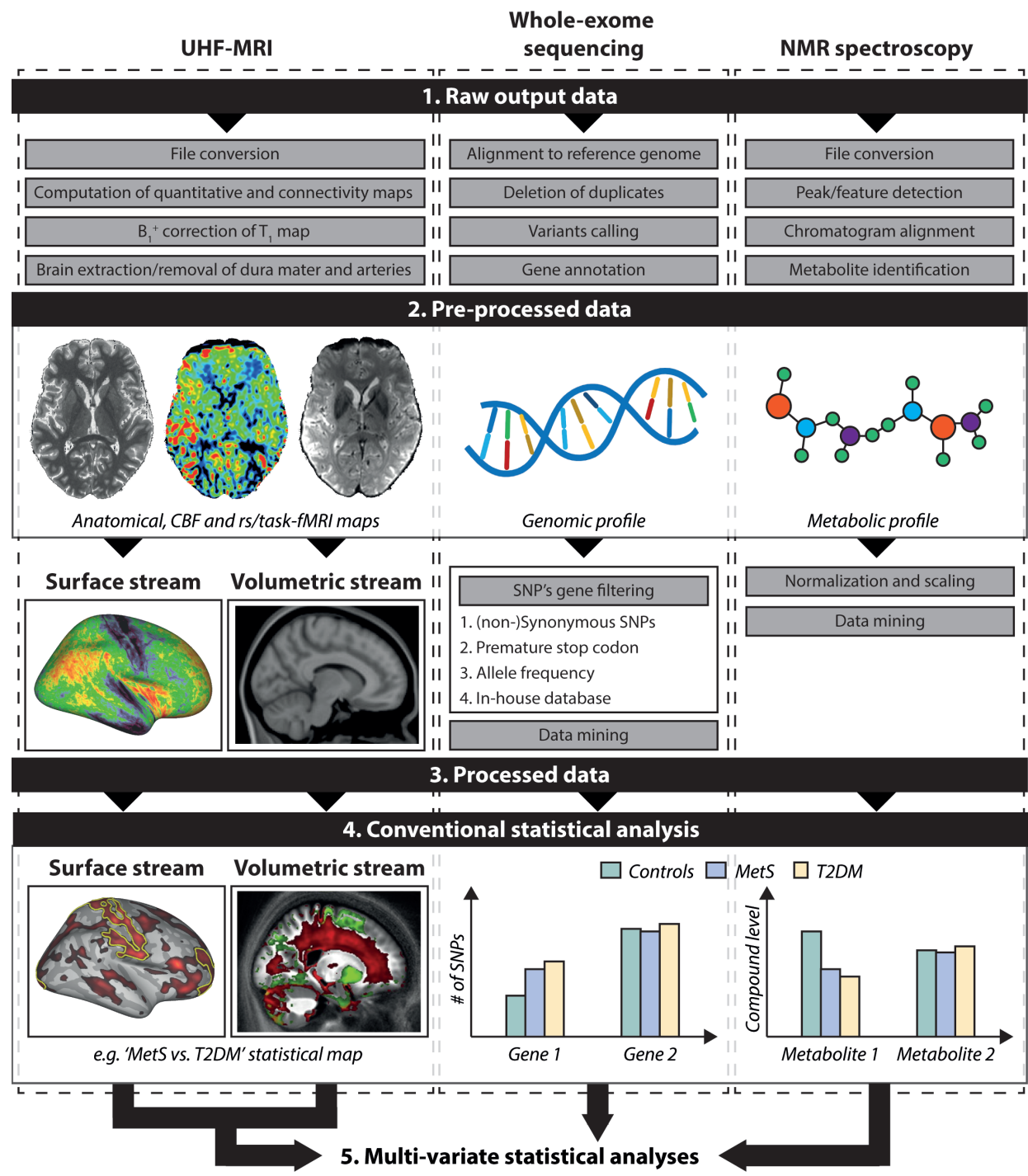

Fig. 5.4. Analysis pipeline. The (1) raw MRI (left), genomic (middle) and metabolomic (right) data are (2-3) (pre)processed before performing (4) conventional and (5) multi-variate statistical analyses. 
portantly, partial volume effects are kept minimal during across-subject coregistration, in contrast with standard voxel-based morphometry (Ashburner and Friston, 2000).

\subsection{3. | Whole-exome sequencing and metabolomics analysis pipeline}

The WES data are processed to obtain data matrices containing the genome of each subject (see Fig. 5.4). To that end, typical workflows were applied, of which the most important steps are briefly highlighted for each method. Note that the metabolomic data were already processed as part of the Maastricht Study. However, for completeness we also briefly describe and display (see Fig. 5.4) the processing steps for the metabolomic data.

\section{Preprocessing}

First, the raw WES output files were analyzed through a standard bioinformatics pipeline, see for example Gerards et al. (2013) and Nguyen et al. (2016). Here, basecalling and demultiplexing was done using bcl2fastq 1.8.4, genetic profiles were aligned onto the human reference genome (hg19) using BWA 0.5.9 (Li and Durbin, 2010), duplicates marked using the PICARD software suite 1.77 (http://picard.sourceforge.net) and variants were called using GATK 2.1-8.6 (McKenna et al., 2010; DePristo et al., 2011). Annotations were added using an in-house-built annotation database, according to the UCSC RefGene track, dbSNP137 and the dbNSFP (v2.0) (Liu et al., 2013). For the metabolomic data, the raw NMR files were converted to workable numerical formats. Peaks/ features (i.e. metabolites) are automatically detected and quantified using standard software tools. The metabolic profiles are aligned and normalized to ensure comparability across profiles.

\section{Processing}

Subsequently, the subject-specific WES data are filtered based on several criteria, for which the stringency depends on the research question(s). For example, to find rare causal variants (Gerards et al., 2013), different thresholds are used for filtering than when interested in common variants (Mahajan et al., 2014). In case of T2DM, variants in different genes will have a small additive or multiplicative effect on the disease phenotype and filtering need to be set up accordingly. Typically, filtering is based on (a) the number of non-synonymous variants, (b) variants leading to a premature stop codon, (c) splice variants with allele frequencies below a certain threshold in dbSNP137 (e.g. in T2DM, this should be relatively lenient) and (d) absence in our in-house database of $\sim 200$ exomes. The remaining variants will be further evaluated for the impact on protein function by SIFT and Polyphen 2 as predicted by the dbNSFP database. Finally, data mining (e.g. clustering) techniques are used to further reduce 


\section{CHAPTER 5}

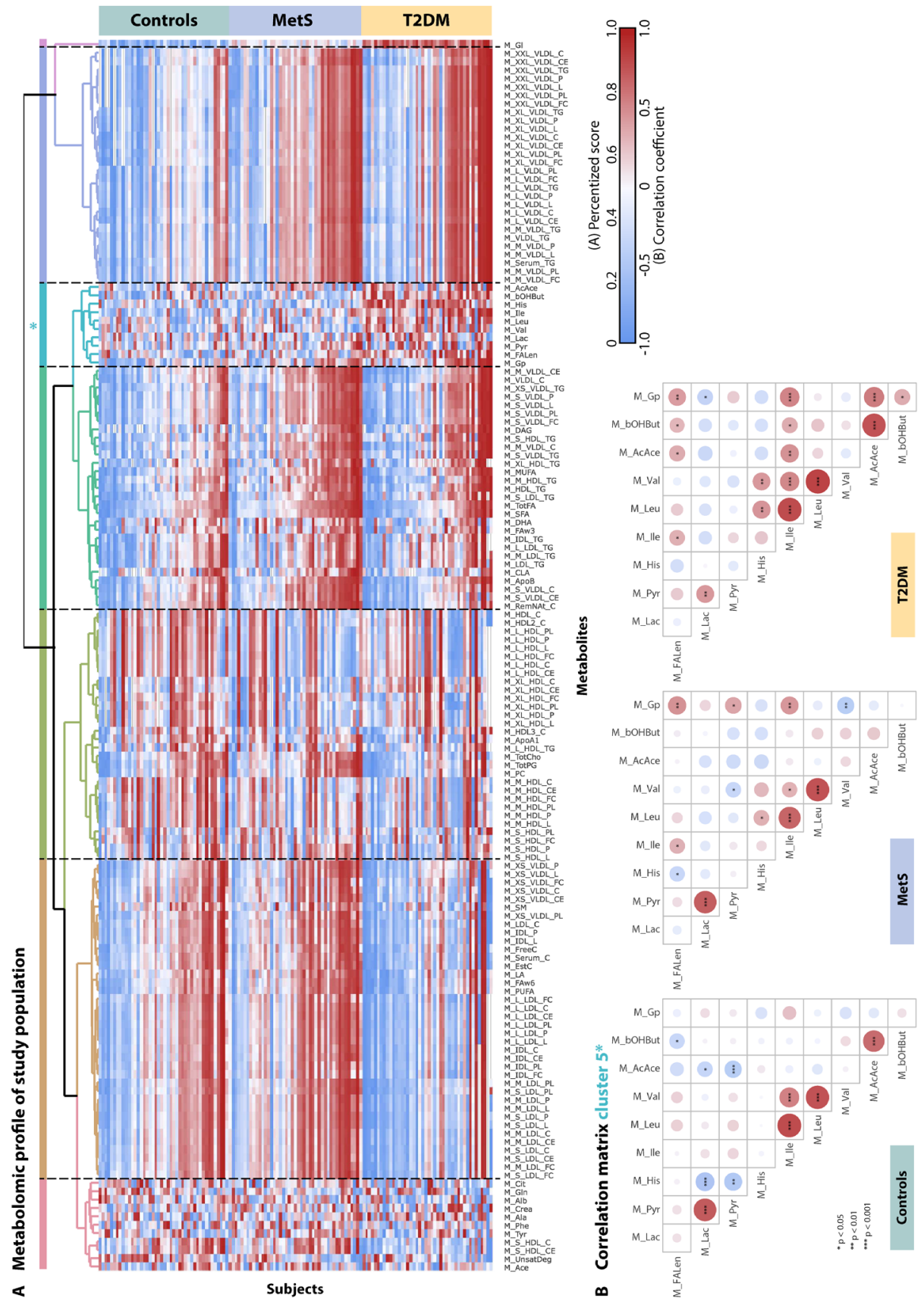

Fig. 5.5. | Example clustermap based on the metabolomic profiles. Metabolites (columns) were hierarchically clustered $\left(N_{\text {clusters }}=7\right.$ ) based on the normalized concentration $(A)$. For example, one clusters of metabolites shows a clear distinct pattern across groups (rows) indicating higher levels of several amino acids, including glutamine, isoleucine and leucine. In addition, comparison of (Pearson's) correlation matrices across groups may reveal additional changes in MetS and T2DM subjects (B). 
the dimensionality of the data for the remaining genes and pre-processed metabolomic data (for example, see Fig. 5.5) (Namkung et al., 2011).

\section{5. | STATISTICAL ANALYSIS STRATEGIES}

So far, several MRI modalities have been combined with genetics, ranging from brain morphometry to functioning, as highlighted by Poline et al. (2015). More importantly, imaging genetics approaches can be used to map the effects of these biological pathways on specific clinical phenotypes (e.g. cardiovascular profiles in our case), but also to highlight genetic variants that put people at risk to develop T2DM, brain abnormalities and/or cognitive decline. However, more elaborate statistical analyses are necessary to identify the causal or significant related genetic variants, especially in such complex diseases such as T2DM, with no prior genetic hypothesis. This is in contrast to the use of simple regression models, such as used in Chapter 4 for the m.3243A>G mutation. As already mentioned before, imaging genetics studies typically require very large sample sizes (i.e. many thousands) due to the large number of associations that can be tested, especially in brain-wide - genome-wide association studies (Vounou et al., 2010). This will be further inflated when additional behavioral or clinical data are included. As such, dimensionality reduction of the data using biological and/or clinical knowledge is necessary to guide the analysis and restrict the number of tests. This can be achieved a priori by reducing the coverage of the acquired data, such as imaging of specific lobes or exomes-restricted sequencing. However, also post-hoc methods can be used. For example, the data's dimensionality can be reduced using data-driven parcellation techniques (see general discussion of this thesis) to define brain regions of interest (Da Mota et al., 2014), metabolite clustering (Forsberg et al., 2018), or selection of genes based on specific SNP'pathogenicity' scores (Kircher et al., 2014). Fig. 5.6 schematically summarizes the possible (statistical) analysis strategies. Here, following the definitions provided in Poline et al. (2015), analyses for imaging genetics studies can be subdivided in either a) mapping (e.g. correlating m.3243A>G mutation load to $G M$ volume, as performed in Chapter 4), b) multivariate (e.g. linking thousands of genetics variants to brain-wide cortical thickness differences between groups, see also Liu and Calhoun (2014)) or c) combined inferences across multiple modalities (e.g. combine information from $\mathrm{T}_{1}, \mathrm{~T}_{2}{ }^{*}$ and cortical thickness to detect genomics-related variation in brain structure). As such, each of these strategies is more applicable for the specific research aims (see Figs. 5.6B and $\mathrm{C}$ ) and will be explored in future analyses. Note that the green and purple arrows in Fig. 5.6A are drawn only between neighboring nodes for simplicity reasons, but may be drawn between every combination of data modalities. 


\section{CHAPTER 5}

A

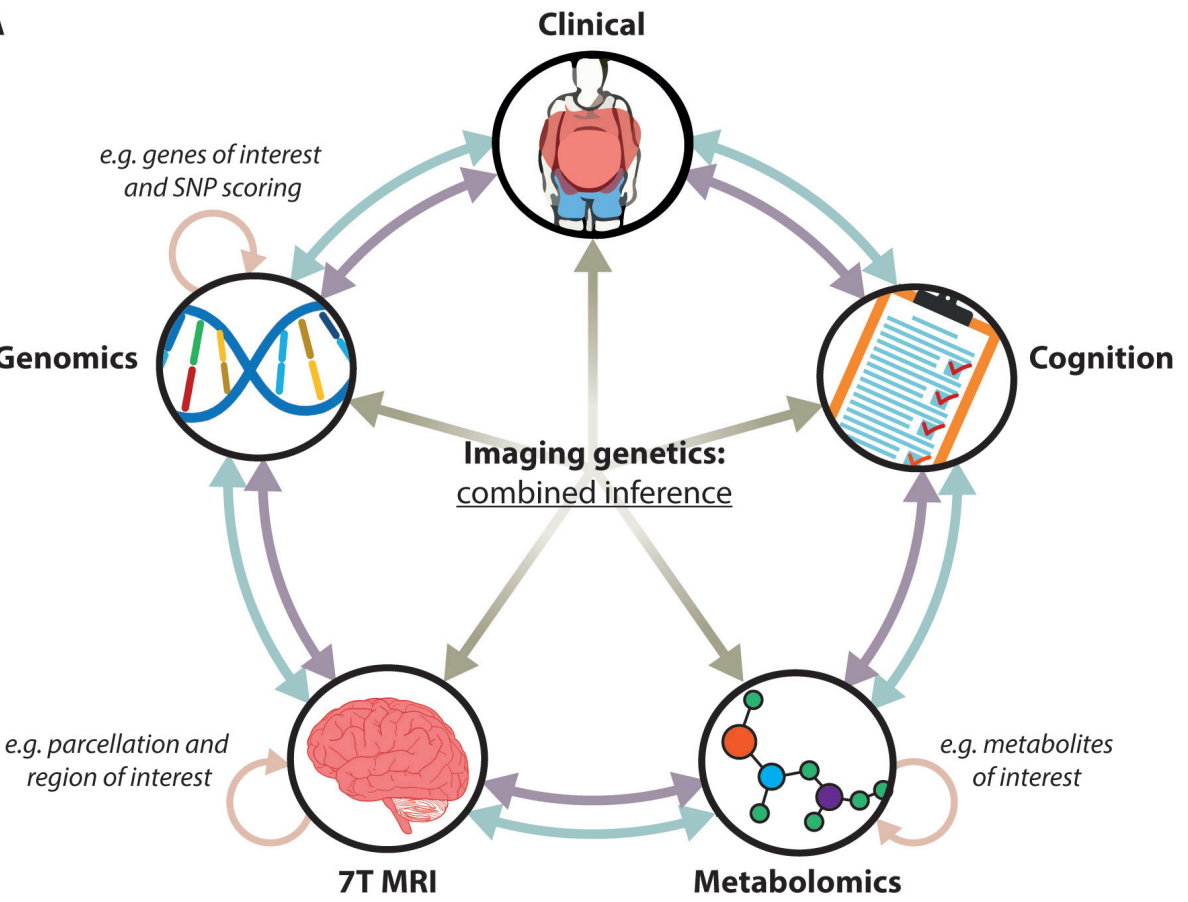

Mapping

Multivariate

Dimensionality reduction

B

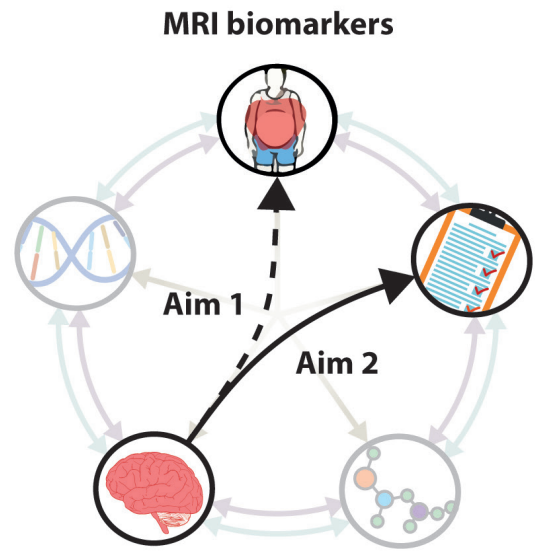

C

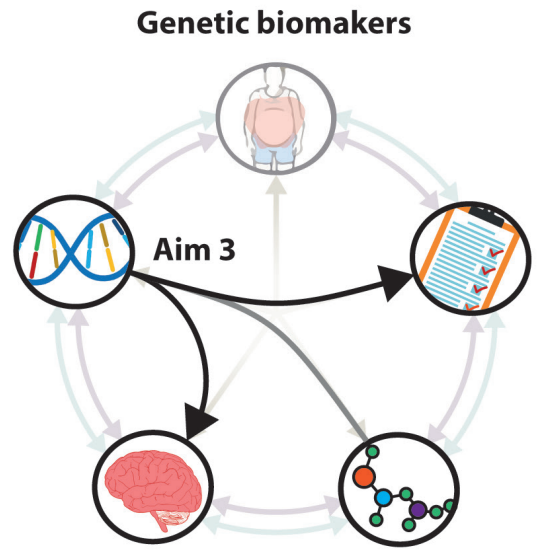

Fig. 5.6. | Schematic outline of the statistical analyses across data modalities. Each combination of two data modalities can be used to 'map' (purple) the association between a single variable from one and many others from the other dataset. Multivariate analysis allows testing for a set of variables from both data modalities, while more than two modalities can be taken into account using combined inference testing (A). Specific approaches that are explored to identify MRI (B) and genetic (C) biomarkers of neurodegeneration and cognitive decline in T2DM. 


\section{6. | CONCLUSION}

Identification of biomarkers that enable characterization of patients at risk to develop T2DM-related pathological features, such as neurodegeneration and cognitive impairment, is crucial to improve prognosis and treatment. Here, imaging genetics has potential applications to study biological pathways from genes (i.e. genetic variants) to specific brain structure (i.e. MRI) and/or function (i.e. cognitive) phenotypes. As such, we acquired 7T MRI and WES data in controls, MetS and T2DM subjects to potentially identify underlying genetic and neurobiological pathways. This dataset, combined with other data modalities, including metabolomics and clinical data, enables a systems biology-wide approach to study the effects of T2DM on the brain. Finally, it allows us to pursue more data modality-individualized research questions. For example, the high resolution anatomical MRI dataset allows quantitative comparisons across subjects and groups to identify T2DM-specific neuroimaging biomarkers.

\section{7. | REFERENCES}

Abu Bakar, M. H., M. R. Sarmidi, K. K. Cheng, A. Ali Khan, C. L. Suan, H. Zaman Huri and H. Yaakob (2015). "Metabolomics - the complementary field in systems biology: a review on obesity and type 2 diabetes." Mol Biosyst 11(7): 1742-1774.

Ashburner, J. (2009). "Computational anatomy with the SPM software." Magn Reson Imaging 27(8): 1163-1174.

Ashburner, J. and K. J. Friston (2000). “Voxel-based morphometry--the methods." Neuroimage 11(6 Pt 1): 805-821.

Barroso, I., J. Luan, R. P. Middelberg, A. H. Harding, P. W. Franks, R. W. Jakes, ... N. J. Wareham (2003). "Candidate gene association study in type 2 diabetes indicates a role for genes involved in beta-cell function as well as insulin action." PLoS Biol 1(1): E20.

Bartels, A. and S. Zeki (2005). "Brain dynamics during natural viewing conditions--a new guide for mapping connectivity in vivo." Neuroimage 24(2): 339-349.

Bazin, P. L., M. Weiss, J. Dinse, A. Schafer, R. Trampel and R. Turner (2014). "A computational framework for ultra-high resolution cortical segmentation at 7Tesla." Neuroimage 93 Pt 2: 201-209.

Biessels, G. J., S. Staekenborg, E. Brunner, C. Brayne and P. Scheltens (2006). "Risk of dementia in diabetes mellitus: a systematic review." Lancet Neurol 5(1): 64-74.

Biessels, G. J., M. W. Strachan, F. L. Visseren, L. J. Kappelle and R. A. Whitmer (2014). “Dementia and cognitive decline in type 2 diabetes and prediabetic stages: towards targeted interventions." Lancet Diabetes Endocrinol 2(3): 246-255.

Biswal, B., F. Z. Yetkin, V. M. Haughton and J. S. Hyde (1995). “Functional connectivity in the motor cortex of resting human brain using echo-planar MRI." Magn Reson Med 34(4): 537-541.

Brundel, M., L. J. Kappelle and G. J. Biessels (2014). "Brain imaging in type 2 diabetes." Eur Neuropsychopharmacol 24(12): 1967-1981.

Brundel, M., M. van den Heuvel, J. de Bresser, L. J. Kappelle, G. J. Biessels and G. Utrecht Diabetic Encephalopathy Study (2010). "Cerebral cortical thickness in patients with type 2 diabetes." J Neurol Sci 299(1-2): 126-130. 


\section{CHAPTER 5}

Bullmore, E. and O. Sporns (2009). "Complex brain networks: graph theoretical analysis of structural and functional systems." Nat Rev Neurosci 10(3): 186-198.

Cameron, A. J., J. E. Shaw and P. Z. Zimmet (2004). "The metabolic syndrome: prevalence in worldwide populations." Endocrinol Metab Clin North Am 33(2): 351-375, table of contents.

Cheng, G., C. Huang, H. Deng and H. Wang (2012). "Diabetes as a risk factor for dementia and mild cognitive impairment: a meta-analysis of longitudinal studies." Intern Med J 42(5): 484-491.

Conijn, M. M., M. I. Geerlings, G. J. Biessels, T. Takahara, T. D. Witkamp, J. J. Zwanenburg, . . . J. Hendrikse (2011). "Cerebral microbleeds on MR imaging: comparison between 1.5 and 7T." AJNR Am J Neuroradiol 32(6): 1043-1049.

Cox, A. J., C. E. Hugenschmidt, L. M. Raffield, C. D. Langefeld, B. I. Freedman, J. D. Williamson, ... D. W. Bowden (2014). "Heritability and genetic association analysis of cognition in the Diabetes Heart Study." Neurobiol Aging 35(8): 1958 e1953-1958 e1912.

Crichton, G. E., M. F. Elias, J. D. Buckley, K. J. Murphy, J. Bryan and V. Frisardi (2012). "Metabolic syndrome, cognitive performance, and dementia." J Alzheimers Dis 30 Suppl 2: S77-87.

Da Mota, B., V. Fritsch, G. Varoquaux, T. Banaschewski, G. J. Barker, A. L. Bokde, . . I. consortium (2014). "Randomized parcellation based inference." Neuroimage 89: 203-215.

de Bresser, J., A. M. Tiehuis, E. van den Berg, Y. D. Reijmer, C. Jongen, L. J. Kappelle, ... G. Utrecht Diabetic Encephalopathy Study (2010). "Progression of cerebral atrophy and white matter hyperintensities in patients with type 2 diabetes." Diabetes Care 33(6): 1309-1314.

DePristo, M. A., E. Banks, R. Poplin, K. V. Garimella, J. R. Maguire, C. Hartl, . . M. J. Daly (2011). "A framework for variation discovery and genotyping using next-generation DNA sequencing data." Nat Genet 43(5): 491-498.

Draganski, B., J. Ashburner, C. Hutton, F. Kherif, R. S. Frackowiak, G. Helms and N. Weiskopf (2011). "Regional specificity of MRI contrast parameter changes in normal ageing revealed by voxel-based quantification (VBQ)." Neuroimage 55(4): 1423-1434.

Eggenschwiler, F., T. Kober, A. W. Magill, R. Gruetter and J. P. Marques (2012). "SA2RAGE: a new sequence for fast B1+ -mapping." Magn Reson Med 67(6): 1609-1619.

Falvey, C. M., C. Rosano, E. M. Simonsick, T. Harris, E. S. Strotmeyer, S. Satterfield, . . A. B. C. S. Health (2013). "Macro- and microstructural magnetic resonance imaging indices associated with diabetes among community-dwelling older adults." Diabetes Care 36(3): 677-682.

Fischl, B. (2012). “FreeSurfer.” Neuroimage 62(2): 774-781.

Florez, J. C., J. Hirschhorn and D. Altshuler (2003). "The inherited basis of diabetes mellitus: implications for the genetic analysis of complex traits." Annu Rev Genomics Hum Genet 4: 257-291.

Ford, E. S., G. Zhao and C. Li (2010). "Pre-diabetes and the risk for cardiovascular disease: a systematic review of the evidence." J Am Coll Cardiol 55(13): 1310-1317.

Forsberg, E. M., T. Huan, D. Rinehart, H. P. Benton, B. Warth, B. Hilmers and G. Siuzdak (2018). “Data processing, multi-omic pathway mapping, and metabolite activity analysis using XCMS Online." Nat Protoc 13(4): 633-651.

Fu, Z., E. R. Gilbert and D. Liu (2013). "Regulation of insulin synthesis and secretion and pancreatic Beta-cell dysfunction in diabetes." Curr Diabetes Rev 9(1): 25-53.

Gami, A. S., B. J. Witt, D. E. Howard, P. J. Erwin, L. A. Gami, V. K. Somers and V. M. Montori (2007). "Metabolic syndrome and risk of incident cardiovascular events and death: a systematic review and meta-analysis of longitudinal studies." J Am Coll Cardiol 49(4): 403-414.

Gerards, M., R. Kamps, J. van Oevelen, I. Boesten, E. Jongen, B. de Koning, ... H. Smeets (2013). "Exome sequencing reveals a novel Moroccan founder mutation in SLC19A3 as a new cause of early-childhood fatal Leigh syndrome." Brain 136(Pt 3): 882-890.

Goodwin, S., J. D. McPherson and W. R. McCombie (2016). “Coming of age: ten years of next-generation sequencing technologies." Nat Rev Genet 17(6): 333-351.

Hayden, E. C. (2014). “Technology: The \$1,000 genome.” Nature 507(7492): 294-295. 
Ivanov, D., B. A. Poser, L. Huber, J. Pfeuffer and K. Uludag (2017). "Optimization of simultaneous multislice EPI for concurrent functional perfusion and BOLD signal measurements at 7T." Magn Reson Med 78(1): 121-129.

Jansen, J. F., F. C. van Bussel, H. J. van de Haar, M. J. van Osch, P. A. Hofman, M. P. van Boxtel, ... W. H. Backes (2016). "Cerebral blood flow, blood supply, and cognition in Type 2 Diabetes Mellitus." Sci Rep 6(1): 10.

Johnson, M. L., N. Parikh, M. E. Kunik, P. E. Schulz, J. G. Patel, H. Chen, ... R. O. Morgan (2012). “Antihypertensive drug use and the risk of dementia in patients with diabetes mellitus." Alzheimers Dement 8(5): 437-444.

Jongen, C., J. van der Grond, L. J. Kappelle, G. J. Biessels, M. A. Viergever, J. P. Pluim and G. Utrecht Diabetic Encephalopathy Study (2007). "Automated measurement of brain and white matter lesion volume in type 2 diabetes mellitus." Diabetologia 50(7): 1509-1516.

Khadka, S., G. D. Pearlson, V. D. Calhoun, J. Liu, J. Gelernter, K. L. Bessette and M. C. Stevens (2016). "Multivariate Imaging Genetics Study of MRI Gray Matter Volume and SNPs Reveals Biological Pathways Correlated with Brain Structural Differences in Attention Deficit Hyperactivity Disorder." Front Psychiatry 7: 128.

Kircher, M., D. M. Witten, P. Jain, B. J. O'Roak, G. M. Cooper and J. Shendure (2014). “A general framework for estimating the relative pathogenicity of human genetic variants." Nat Genet 46(3): 310-315.

Lamport, D. J., C. L. Lawton, M. W. Mansfield and L. Dye (2009). "Impairments in glucose tolerance can have a negative impact on cognitive function: a systematic research review." Neurosci Biobehav Rev 33(3): 394-413.

Last, D., D. C. Alsop, A. M. Abduljalil, R. P. Marquis, C. de Bazelaire, K. Hu, .. . V. Novak (2007). "Global and regional effects of type 2 diabetes on brain tissue volumes and cerebral vasoreactivity." Diabetes Care 30(5): 1193-1199.

Li, C., E. S. Ford, G. Zhao and A. H. Mokdad (2009). “Prevalence of pre-diabetes and its association with clustering of cardiometabolic risk factors and hyperinsulinemia among U.S. adolescents: National Health and Nutrition Examination Survey 2005-2006." Diabetes Care 32(2): 342-347.

$\mathrm{Li}, \mathrm{H}$. and R. Durbin (2010). "Fast and accurate long-read alignment with Burrows-Wheeler transform." Bioinformatics 26(5): 589-595.

Liu, J. and V. D. Calhoun (2014). "A review of multivariate analyses in imaging genetics." Front Neuroinform 8: 29.

Liu, X., X. Jian and E. Boerwinkle (2013). "dbNSFP v2.0: a database of human non-synonymous SNVs and their functional predictions and annotations." Hum Mutat 34(9): E2393-2402.

Lorenzo, C., M. Okoloise, K. Williams, M. P. Stern, S. M. Haffner and S. San Antonio Heart (2003). "The metabolic syndrome as predictor of type 2 diabetes: the San Antonio heart study." Diabetes Care 26(11): 3153-3159.

Maassen, J. A., T. H. LM, E. Van Essen, R. J. Heine, G. Nijpels, R. S. Jahangir Tafrechi, ... H. H. Lemkes (2004). "Mitochondrial diabetes: molecular mechanisms and clinical presentation." Diabetes 53 Suppl 1: S103-109.

Mahajan, A., M. J. Go, W. Zhang, J. E. Below, K. J. Gaulton, T. Ferreira, ... A. P. Morris (2014). "Genome-wide trans-ancestry meta-analysis provides insight into the genetic architecture of type 2 diabetes susceptibility." Nat Genet 46(3): 234-244.

Marques, J. P., T. Kober, G. Krueger, W. van der Zwaag, P. F. Van de Moortele and R. Gruetter (2010). "MP2RAGE, a self bias-field corrected sequence for improved segmentation and T1-mapping at high field." Neuroimage 49(2): 1271-1281.

McKenna, A., M. Hanna, E. Banks, A. Sivachenko, K. Cibulskis, A. Kernytsky, . . . M. A. DePristo (2010). "The Genome Analysis Toolkit: a MapReduce framework for analyzing next-generation DNA sequencing data." Genome Res 20(9): 1297-1303.

Moran, C., T. G. Phan, J. Chen, L. Blizzard, R. Beare, A. Venn, ... V. Srikanth (2013). “Brain atrophy in 


\section{CHAPTER 5}

type 2 diabetes: regional distribution and influence on cognition." Diabetes Care 36(12): 40364042.

Morris, A. P., B. F. Voight, T. M. Teslovich, T. Ferreira, A. V. Segre, V. Steinthorsdottir, .. C. Meta-analysis (2012). "Large-scale association analysis provides insights into the genetic architecture and pathophysiology of type 2 diabetes." Nat Genet 44(9): 981-990.

Musen, G., A. M. Jacobson, N. R. Bolo, D. C. Simonson, M. E. Shenton, R. L. McCartney, . . W. S. Hoogenboom (2012). "Resting-state brain functional connectivity is altered in type 2 diabetes." Diabetes 61(9): 2375-2379.

Namkung, J., P. Raska, J. Kang, Y. Liu, Q. Lu and X. Zhu (2011). “Analysis of exome sequences with and without incorporating prior biological knowledge." Genet Epidemiol 35 Suppl 1: S48-55.

Nguyen, M., I. Boesten, D. M. Hellebrekers, J. Vanoevelen, R. Kamps, B. de Koning, ... H. J. Smeets (2016). "Pathogenic CWF19L1 variants as a novel cause of autosomal recessive cerebellar ataxia and atrophy." Eur J Hum Genet 24(4): 619-622.

Padberg, I., E. Peter, S. Gonzalez-Maldonado, H. Witt, M. Mueller, T. Weis, .. . P. Schatz (2014). "A new metabolomic signature in type-2 diabetes mellitus and its pathophysiology." PLoS One 9(1): e85082.

Pe'er, I., R. Yelensky, D. Altshuler and M. J. Daly (2008). “Estimation of the multiple testing burden for genomewide association studies of nearly all common variants." Genet Epidemiol 32(4): 381-385.

Pohmann, R., O. Speck and K. Scheffler (2016). "Signal-to-noise ratio and MR tissue parameters in human brain imaging at 3, 7, and 9.4 tesla using current receive coil arrays." Magn Reson Med 75(2): 801-809.

Poline, J.-B., J. Breeze and V. Frouin (2015). Imaging genetics with fmri. fMRI: From Nuclear Spins to Brain Functions, Springer: 699-738.

Rao Kondapally Seshasai, S., S. Kaptoge, A. Thompson, E. Di Angelantonio, P. Gao, N. Sarwar, .. .C. Emerging Risk Factors (2011). "Diabetes mellitus, fasting glucose, and risk of cause-specific death." N Engl J Med 364(9): 829-841.

Reijmer, Y. D., E. van den Berg, C. Ruis, L. J. Kappelle and G. J. Biessels (2010). “Cognitive dysfunction in patients with type 2 diabetes." Diabetes Metab Res Rev 26(7): 507-519.

Roberts, L. D., A. Koulman and J. L. Griffin (2014). "Towards metabolic biomarkers of insulin resistance and type 2 diabetes: progress from the metabolome." Lancet Diabetes Endocrinol 2(1): 65-75.

Robinson, E. C., S. Jbabdi, M. F. Glasser, J. Andersson, G. C. Burgess, M. P. Harms, ... M. Jenkinson (2014). "MSM: a new flexible framework for Multimodal Surface Matching." Neuroimage 100: 414-426.

Sarwar, N., P. Gao, S. R. Seshasai, R. Gobin, S. Kaptoge, E. Di Angelantonio, ... J. Danesh (2010). "Diabetes mellitus, fasting blood glucose concentration, and risk of vascular disease: a collaborative meta-analysis of 102 prospective studies." Lancet 375(9733): 2215-2222.

Schram, M. T., S. J. Sep, C. J. van der Kallen, P. C. Dagnelie, A. Koster, N. Schaper, ... C. D. Stehouwer (2014). "The Maastricht Study: an extensive phenotyping study on determinants of type 2 diabetes, its complications and its comorbidities." Eur J Epidemiol 29(6): 439-451.

Soininen, P., A. J. Kangas, P. Wurtz, T. Suna and M. Ala-Korpela (2015). “Quantitative serum nuclear magnetic resonance metabolomics in cardiovascular epidemiology and genetics." Circ Cardiovasc Genet 8(1): 192-206.

Spauwen, P. J., S. Kohler, F. R. Verhey, C. D. Stehouwer and M. P. van Boxtel (2013). “Effects of type 2 diabetes on 12-year cognitive change: results from the Maastricht Aging Study." Diabetes Care 36(6): 1554-1561.

Stuber, C., M. Morawski, A. Schafer, C. Labadie, M. Wahnert, C. Leuze, .. R. Turner (2014). “Myelin and iron concentration in the human brain: a quantitative study of MRI contrast." Neuroimage 93 Pt 1: 95-106. 
Stumvoll, M., B. J. Goldstein and T. W. van Haeften (2005). "Type 2 diabetes: principles of pathogenesis and therapy." Lancet 365(9467): 1333-1346.

Temelkova-Kurktschiev, T. and T. Stefanov (2012). "Lifestyle and genetics in obesity and type 2 diabetes." Exp Clin Endocrinol Diabetes 120(1): 1-6.

Theysohn, J. M., O. Kraff, S. Maderwald, M. Barth, S. C. Ladd, M. Forsting, ... E. R. Gizewski (2011). "7 tesla MRI of microbleeds and white matter lesions as seen in vascular dementia." J Magn Reson Imaging 33(4): 782-791.

Umemura, T., T. Kawamura, H. Umegaki, S. Mashita, A. Kanai, T. Sakakibara, ... G. Sobue (2011).“Endothelial and inflammatory markers in relation to progression of ischaemic cerebral small-vessel disease and cognitive impairment: a 6-year longitudinal study in patients with type 2 diabetes mellitus." J Neurol Neurosurg Psychiatry 82(11): 1186-1194.

van Bussel, F. C., W. H. Backes, T. M. van Veenendaal, P. A. Hofman, M. P. van Boxtel, M. T. Schram, . .. J. F. Jansen (2016). “Functional Brain Networks Are Altered in Type 2 Diabetes and Prediabetes: Signs for Compensation of Cognitive Decrements? The Maastricht Study." Diabetes 65(8): 24042413.

van Bussel, F. C. G., W. H. Backes, P. A. M. Hofman, R. J. van Oostenbrugge, M. P. J. van Boxtel, F. R. J. Verhey, ... J. F. A. Jansen (2017). “Cerebral Pathology and Cognition in Diabetes: The Merits of Multiparametric Neuroimaging." Front Neurosci 11: 188.

van Elderen, S. G., A. de Roos, A. J. de Craen, R. G. Westendorp, G. J. Blauw, J. W. Jukema, .. J. van der Grond (2010). "Progression of brain atrophy and cognitive decline in diabetes mellitus: a 3-year follow-up." Neurology 75(11): 997-1002.

Venter, J. C., M. D. Adams, E. W. Myers, P. W. Li, R. J. Mural, G. G. Sutton, . . X. Zhu (2001). “The sequence of the human genome." Science 291(5507): 1304-1351.

Vounou, M., T. E. Nichols, G. Montana and I. Alzheimer's Disease Neuroimaging (2010). "Discovering genetic associations with high-dimensional neuroimaging phenotypes: A sparse reduced-rank regression approach." Neuroimage 53(3): 1147-1159.

Weir, G. C. and S. Bonner-Weir (2004). "Five stages of evolving beta-cell dysfunction during progression to diabetes." Diabetes 53 Suppl 3: S16-21.

Wild, S., G. Roglic, A. Green, R. Sicree and H. King (2004). “Global prevalence of diabetes: estimates for the year 2000 and projections for 2030." Diabetes Care 27(5): 1047-1053. 
SUMMARY AND GENERAL DISCUSSION 
A growing amount of evidence shows that clinical, but also fundamental neuroscientific studies benefit from ultra-high field MRI (Trattnig et al., 2016; Ugurbil, 2017). Because of the higher SNR (Pohmann et al., 2016), 7T MRI is able to provide anatomical and functional images with great detail and contrast, without jeopardizing patient comfort and, hereby, outperforming field strengths that are typically used in the clinic $(\leq 3 \mathrm{~T})$. As such, the imaging community is pushing ultra-high field MRI forward, which recently led to the CE certificate and FDA approval for the Siemens 7T Magnetom Terra scanner, paving the way for clinical use in Europe and North America, respectively. The number of research centers that operate 7T MRI scanners has steadily increased in the last years and has led to tremendous improvements in hardware, instrumentation and sequence design (Ugurbil, 2017; Polimeni and Uludağ, 2018). Consequentially, it becomes possible to acquire high quality, high resolution, multi-parametric data within an hour. Depending on the specific research aims and/or study population, several choices have to be made for the design of the imaging protocol: (i) weighted or quantitative imaging (not mutually exclusive), (ii) type of contrasts (e.g. $T_{1}, T_{2}{ }^{(*)}$, MT, perfusion, diffusion, $\mathrm{fMRI}$ ), (iii) spatial (and temporal) resolution and (iv) spatial coverage (Marques and Norris, 2017). Throughout the current thesis, we explored the different properties and applications of a multi-parameter, quantitative protocol in healthy subjects and in metabolic diseases. This protocol was developed to capture the biochemical (i.e. microstructure) and metabolic (i.e. blood flow) properties of the brain. In this chapter, we first briefly summarize the work presented in this thesis, followed by a discussion on several related issues and developments that are central to consider for a more broad use of the protocol. Finally, we conclude with a description of a potential application and work in progress not explored in this thesis.

\section{1. | SUMMARY}

The MP2RAGE sequence is widely used for anatomical imaging at 7T (Marques et al., 2010). Initially developed on the Siemens system platform, it has also been extended towards other 7T vendors (Mougin et al., 2016). In addition to the standard acquisition of a $T_{1} w$ image, a quantitative $T_{1}$ map is obtained in this approach, allowing studying brain microstructure besides morphology. However, $\mathrm{B}_{1}{ }^{+}$-related image artifacts - too strong to correct by using dielectric pads (Teeuwisse et al., 2012) and specialized adiabatic inversion pulses (Hurley et al., 2010) - hamper accurate analyses in the affected regions. Especially brain regions that suffer from severe local field inhomogeneity effects, such as the inferior temporal and frontal lobes, are difficult to properly segment into white (WM), gray matter (GM) and cerebrospinal fluid (CSF). As a result, tedious manual corrections of the automatic image segmentations are necessary to 


\section{CHAPTER 6}

correct for these errors and causes excessive amounts of work, in particular in largescale imaging studies. Therefore, in Chapter 2, we explored the benefits on cortical thickness measurements and $\mathrm{T}_{1}(\mathrm{w})$ contrast when the $\mathrm{B}_{1}{ }^{+}$-related variations across the cortex are taken into account using the Sa2RAGE $B_{1}^{+}$map. By doing so, substantial improvement in the delineation between WM, GM and CSF could be achieved due to the locally improved contrast between tissue types. This resulted in more accurate cortical thickness measurements, shown by the increased correspondence with a normative dataset. In addition, we showed that the cortical $\mathrm{T}_{1}$ values were significantly biased by the non-uniform RF transmit efficiency prior to the post-hoc $\mathrm{B}_{1}^{+}$correction, leading to artificially increased variation across the cortex. The latter phenomenon was significantly reduced after the correction, revealing the typical myelin-related pattern observed in earlier and lower field strength studies (Glasser and Van Essen, 2011; Lutti et al., 2014). Similar improvements (e.g. increased inter-tissue type contrast) has also been shown for the subcortical GM structures (Marques and Gruetter, 2013). Taken together, these results have important implications for future studies that use the MP2RAGE data for morphological and/or microstructural analyses. Similar biases are also expected for other $T_{1}$ acquisition approaches (e.g. also in the standard $T_{1} w$ images using MPRAGE, the WM/GM contrast is low in the anterior temporal lobe). Finally, supported by its easy implementation and short scanning duration, this correction step should be part of any users' MP2RAGE data pre-processing workflow.

Following up on the work from the previous chapter to obtain more accurate surface reconstructions of the brain, Chapter $\mathbf{3}$ compares the performance of several popular myelin-based mapping approaches using anatomical 7T data. Here, reproducibility and reliability of weighted and quantitative $T_{1}$ (i.e. corrected for the $\mathrm{B}_{1}{ }^{+}$bias field, explained above), $\mathrm{T}_{2}{ }^{*}$ and their ratio were compared in terms of contrast-to-noise ratio (CNR), parcellation reliability (i.e.'parcellability'), and inter- and intra-subject variability and reproducibility. Quantitative MRI methods are considered more robust compared to their weighted counterparts in terms of non-biochemical influences, due to their dominant dependence on intrinsic tissue properties, such as longitudinal $\left(T_{1}\right)$ or transversal relaxation times $\left(\mathrm{T}_{2}{ }^{*}\right)$. In contrast, weighted imaging is additionally affected by non-biochemical factors, such as sensitivity to MRI acquisition parameters and image imperfections (e.g. $B_{0}$ and $B_{1}$ inhomogeneities). As such, quantitative MRI allows more reliable and biology-relevant comparisons within subjects and between healthy subjects and patients. However, while both types of contrasts (i.e. quantitative vs. weighted) may be appropriate to accurately capture the variation of myelin and iron concentration across the cortex within a subject (but not necessarily across subjects), they were previously not systematically compared at 7T. In our study, similar but not 
identical myelination patterns were observed across all tested contrasts. However, we found that CNR per unit time and parcellability were lower for the transversal compared to the longitudinal relaxation parameters. In addition, quantitative $R_{1}\left(=1 / T_{1}\right)$ turned out to be the most reliable parameter to map the myelin distribution compared to the other parameters. The respective maps were characterized by the lowest inter- and intra-subject coefficient of variation. In addition, these spatial surface maps can be used for microstructure-informed delineation of cortical areas. We explored two area-defining methods: (i) thresholding the MR parameter values and (ii) surface gradients of these values, to determine areal borders based on the cortical surface pattern. Both methods are partially observer-dependent, needing manual interaction (i.e., choice of threshold or connecting high gradient values) to provide unambiguous borders. In order to obtain fully data-driven cortical parcellations and to overcome this observer-dependency, several methods have been developed in this emerging field. We will briefly discuss this topic later in this chapter and show some examples.

Similar to the studies presented in Chapters 2 and 3, we acquired quantitative MRI data, additionally including cerebral blood flow (CBF), in metabolic diseases. Here, the MRI data were combined with genetic analyses in order to relate morphological-, microstructural- and metabolic-related variations in the brain, measured using MRI, to genetic differences across the study population (i.e. 'imaging genetics'). This was performed in a metabolic disease, for which the genetic underpinning is well established and only the mutation load varies between patients (Chapter 4) and in Type 2 Diabetes Mellitus (T2DM), a more complex polygenetic disorder (Chapter 5). In Chapter 4, we examined patients with m.3243A>G mutation; this mutation affects the mitochondrial DNA and leads to the expression of a widely varying phenotype across the patient population. Symptoms often observed include hearing loss, development of (mitochondrial) diabetes, muscle atrophy, as well as tissue changes in the brain. Interestingly, the number of mutated mitochondria in a single cell, referred to as 'mutation load', differs across tissue types (i.e. blood and urine epithelial cells), but also between patients. Previous studies have shown that the disease severity positively correlates with the mutation load measured across several tissue types. However, none of them investigated potential brain correlates. Therefore, the aim of the small-scale imaging genetics study was to assess to this relation using the mutation load in blood and urine. In our patient population, the mutation loads in blood and urine significantly correlated with disease severity, confirming the observations in earlier studies. We found a strong correlation between brain volume and both mutation loads, however, more strongly for that measured in blood. Cortical GM, WM and cerebellar GM volume significantly decreased with increasing mutation load. Interestingly, subcortical GM 


\section{CHAPTER 6}

(i.e. putamen and globus pallidus) volume was lower for the m.3243A $>$ G patients compared to controls, but no mutation load effect was observed. In addition, clusters of vertices were identified, where cortical thickness significantly decreased, while $T_{1}, T_{2}^{*}$ and CBF tend to increase as function of mutation load. These clusters spanned specific areas involved in auditory processing (e.g. auditory cortex) and those that are characterized by a higher resting-state connectivity (e.g. default-mode and default-attention networks). These observations can be linked to the clinical symptoms in m.3243A>G patients, such as hearing loss and decreased attentional/executive functioning. These results may guide future $\mathrm{MRI}$, but also in vitro, studies to track disease pathogenesis and relationship with specific clinical symptoms.

While the work and analyses in Chapter 4 are straightforward, the population and analysis strategies presented in Chapter $\mathbf{5}$ are more challenging. In this chapter, the main focus was to highlight the different types of data and discuss potential analyses strategies, in addition to providing a brief description of the preliminary output from the proposed analyses workflows. The clinical characteristics of the study population confirmed the significant differences in the cardiovascular profiles (e.g. blood glucose and lipid levels) across groups, which was expected based on the group-specific inclusion criteria. Ultimately, the data will be analyzed from different perspectives with the aim to develop multi-level biomarkers in metabolic syndrome (MetS) and T2DM. Due to the complex genetic nature of T2DM, the high dimensionality of the data, and the possibility to merge multiple data modalities (i.e. MRI, genetic, metabolomic, cognitive and clinical), more advanced statistical methods have to be utilized to identify T2DM biomarkers. For this, the availability of each dataset can be used to good advantage. For example, we showed a cluster of metabolites that were present in distinct quantities in T2DM subjects compared to controls. Therefore, characterization of the related metabolic pathways, based on this data, may provide clues for narrowing down analyses, and formulation of specific hypotheses with regards to the whole-exome sequencing data.

While interesting from an imaging genetics or 'imaging -omics' point of view, the presented dataset also provides opportunities to explore each data modality separately. Genotype-phenotype relationships, as well as metabolomic profiles, can be established to define modality-specific biomarkers that potentially might aid the explanation of phenotypical and functional differences across subjects/groups. However, the sample size $(\mathrm{N}=133)$ - even though high for a 7T study - is rather limited for genetic and metabolomic studies, for which sample sizes of $>1000$ are preferred. Dimensionality reduction/feature selection (i.e. filtering) techniques, such as independent 
component analysis, but also methods using prior knowledge (Kircher et al., 2014), become more important (Saeys et al., 2007). This is less crucial for the MRI part, as it represents a rich and unique dataset that can be used to answer several modality-specific research questions. For example, the submillimeter resolution of the anatomical data provides spatial details that can be used to identify small-scale structural changes in the brain as a result of impaired glucose metabolism. This includes changes in tissue susceptibility due to cerebral microbleeds or iron depositions. Also more methodologically relevant questions can be answered, such as the relationship between blood $\mathrm{T}_{1}$ and hematocrit levels and how this changes in T2DM, an issue important for the quantification of cerebral blood flow using ASL (Xu et al., 2018). Across all applications of 7T MRI described in this thesis, it is important to monitor and evaluate data quality, crucial for implementing these approaches in clinical populations. Therefore, in the following, we will address some of the issues encountered for the different imaging contrasts and describe potential technological developments that may be able to overcome these.

\section{2. | STANDARDIZATION AND HARMONIZATION OF CLINICAL QUANTITATIVE 7T MRI}

It has been highlighted throughout this thesis that $7 \mathrm{~T}$ imaging provides a number of advantages compared to lower field strengths. In addition, the presented data demonstrates the possibility to acquire whole-brain high resolution anatomical, perfusion and functional data within an hour and its application to study neurodegeneration in a clinical population. However, the potential of $7 \mathrm{~T}$ reaches far beyond what is demonstrated in this thesis, including fMRI (Uludag and Blinder, 2017), spectroscopy (Bogner et al., 2011), diffusion (Vu et al., 2015) and vascular imaging (De Cocker et al., 2016). The addition of such measurements will complement our protocol and boosts the range of applications it can be used for. Quality assessment, standardization and harmonization of MRI protocols are essential in order to allow utilization and generalization of such protocols across studies, imaging sites and scanner vendors.

Acquisition of large-scale datasets, too expensive to cover by individual institutions, as well as those in rare diseases, such as in $\mathrm{m} .3243 \mathrm{~A}>\mathrm{G}$ patients, can be promoted due to the possibilities to pool data across multiple imaging sites. Several nation-wide and international $7 \mathrm{~T}$ networks have been established to serve this purpose, such as the German Ultra High Field Imaging (GUFI) and United Kingdom's 7T (UK7T) networks and European Ultra-high field Imaging Network for Neurodegenerative Diseases (EUFIND). These consortia aim to set up standardized sequences across the main 7T suppliers that limit the effect of hard- (e.g. coils and gradients) and software (e.g. imaging 


\section{CHAPTER 6}

sequence implementations and reconstruction methods) differences. Sequences and corresponding parameters need to be chosen in such a way that they are applicable across Siemens, Philips and General Electric (GE) systems and provide comparable data in terms of temporal and spatial SNR and CNR. Here, it is important to find the right balance between conformity across vendors and maximal performances within vendor (i.e. not necessarily accepting a 'least common denominator' solution). The generalizability of clinical MRI depends on the comparability of the data generated at different sites, scanner vendors and time points (Voelker et al., 2016). Quantitative MRI (ideally) overcomes potential, non-biochemical inter-site, intra-subject biases that are present in weighted MRI data, which hinder the direct comparison across studies and between patients and healthy controls. Hereafter, we will address some of the quality-related issues that were encountered while acquiring and analyzing the presented data.

\subsection{1. | Anatomical imaging}

The results in Chapters 2 and 3 demonstrated that the quality of anatomical data acquired at 7T depends on the type of contrast. Here, quantitative $T_{1}$ maps were more robust across subjects and within subjects, compared to their weighted counterparts. However, in order to obtain more precise $T_{1}$ maps, we show that homogeneous RF transmit profiles are important, even for quantitative data. To limit possible confounding effects due to this, sequence (i.e. MP2RAGE, in this case) parameters can be optimized in such a way that the $\mathrm{B}_{1}{ }^{+}$dependency is decreased or, alternatively, CNR is increased, depending on the specific study aim (Marques and Gruetter, 2013). In contrast, quantitative $\mathrm{T}_{2}{ }^{*}$ maps were characterized by a much higher variation and sensitivity for non-biochemical variation, in line with previous findings at lower field strengths (Weiskopf et al., 2013). This lower reproducibility can be partly ascribed to susceptibility artifacts near the inferior temporal and frontal regions. Although lower in high resolution data, due to smaller intra-voxel spin phase coherence loss, also SNR differences across echo times may lead to noise enhancement when fitting the $\mathrm{T}_{2}{ }^{*}$-weighted data. Inclusion of more echo times, and potentially, a longer maximal echo time may improve the precision of the $\mathrm{T}_{2}{ }^{*}$ maps, at the cost of prolonging acquisition times (Cohen-Adad, 2014; Govindarajan et al., 2015). This would additionally enable the use of more advanced (i.e. mono-exponential vs. multi-exponential) fitting models to gain quantification accuracy and biochemical specificity (van Gelderen et al., 2012). Alternatively, $T_{2}$ (i.e. transversal relaxation time for spin-echo sequences, less affected by local field inhomogeneities), instead of $\mathrm{T}_{2}{ }^{*}$, imaging, may yield more reproducible data. However, as for now, acquisition of whole-brain $\mathrm{T}_{2}$-weighted images at 7T remains challenging due to inhomogeneous transmit profiles, power deposition 
limitations and long acquisition times using conventional single-channel transmission. In addition, $\mathrm{T}_{2}{ }^{*}$ maps may be more biochemically informative, due to its higher dependency on local changes in iron concentration (Cohen-Adad, 2014; Stuber et al., 2014).

\subsection{2. | Perfusion imaging}

While anatomical and functional imaging at 7T benefit from ultra-high field strengths, this is less evident for ASL imaging. Compared to the $T_{1}$ and $T_{2}{ }^{*}$ maps, $A S L$ data presumably harbors lower SNR and CNR. Although improvements were observed compared to lower fields strengths, in particular at higher spatial resolutions (Gardener et al., 2009; Ivanov et al., 2017), ASL suffers from persisting problems (Teeuwisse et al., 2010). The upper limit of ASL SNR is determined by the low GM microvascular density ( 1\%-2\% of local tissue volume) and signal reduction due to $T_{1}$ recovery after labeling. Especially for clinical purposes, when scanning time is a limiting factor, increasing the SNR is challenging. In addition, acquisition of whole-brain CBF maps remains an issue, as well as the sensitivity of CBF acquisitions to subject motion. Several possible practical solutions have been explored to maximize data quality. These include, for example, the use of dielectric pads, and optimized positioning of the head and magnet isocenter reference to improve to $\mathrm{B}_{1}{ }^{+}$homogeneity and labeling efficiency, respectively (Ghariq et al., 2012). Also, several improvements in sequence design have been proposed to increase coverage without increasing scan time. This includes the implementation of echo-planar imaging (EPI) readouts, parallel (e.g. generalized autocalibrating partially parallel acquisitions, GRAPPA), simultaneous multi-slice (SMS, which also reduces motion sensitivity) or multi-band (MB) imaging (Feinberg et al., 2013; Kim et al., 2013; Ivanov et al., 2017). While these methods may lead to losses in (temporal) SNR, the sequence parameters in the current protocol were chosen such that the loss in ( $t$ )SNR was kept minimal for maximal brain coverage ( 80\%) (see also Ivanov et al. (2017) and references herein for more details and an in-depth discussion of ASL at UHF). To conclude, ASL is a promising tool to measure brain perfusion non-invasively; however, future studies and technical developments (see below) are necessary to reach its full potential at UHF.

\section{3. | TECHNICAL DEVELOPMENTS TO IMPROVE CLINICAL APPLICABILITY OF 7T}

Several technical developments are currently being explored to improve the clinical applicability of 7T imaging and to overcome (some of) the limitations described above. This includes, for example, prospective motion correction. Prospective motion correc- 


\section{CHAPTER 6}

tion can be used for real-time updating of most pulse sequences to reduce artifacts caused by head motion and therefore it has potential to make a large impact in clinical routine (Maclaren et al., 2013). In addition, magnetic field monitoring, to compensate for spatiotemporal field perturbations, is another promising technique to reduce image artifacts and distortion (Barmet et al., 2008). Field control improved the fidelity of $\mathrm{T}_{2}{ }^{*}$ maps by mitigating field changes related to the subject's breathing pattern, for example (Wyss et al., 2017).

The introduction of the parallel (i.e. multi-channel) transmission (pTx) technology has been one of the main developments for UHF imaging. It may resolve some, if not many, of the issues discussed above, by improving the temporal resolution and energy efficiency of the radio-frequency (RF) pulses (Katscher et al., 2003; Zhu, 2004). Advanced coil designs (Shajan et al., 2014; Sengupta et al., 2016) and RF pulses (Setsompop et al., 2008; Poser et al., 2014) are being developed to take full advantage of the technology. Shorter excitations can be achieved without risking data quality reduction, which is particularly beneficial for clinical purposes. Alternatively, it can be used to reduce $\mathrm{B}_{1}{ }^{+}$ inhomogeneities and resulting image contrast biases across and within subjects (such as observed in Chapters 2 and 3) and imaging sites, without increasing scan duration (Cloos et al., 2012). In addition, ASL techniques will benefit from parallel transmission by enabling a more tailored labeling field maximizing labeling efficiency (Oliver-Taylor et al., 2012). So far, the majority of current pTx-related studies depended mostly on custom hard- (e.g. coils) and software (e.g. sequences) not publicly available and only little work has been transferred into clinical practice, although clinical applications of dual-transmit systems have been discussed (Brink et al., 2015). Future work is necessary to further assess the advantages of pTx and improve its workflow for clinical routine, for example, by limiting the required user interaction and computational complexity.

\section{4. | CLINICAL INTERPRETATION OF QUANTITATIVE 7T MRI}

Given the increased spatial resolution and contrast that can be obtained using (quantitative) $7 \mathrm{~T} \mathrm{MRI}$, better understanding of the biophysical mechanisms and their relation to pathologies, are necessary to improve clinical decision-making. This requires interdisciplinary research to link, for example, changes in tissue composition (e.g. studied by biologists and chemists), due to disease (e.g. pathologists), to differences of the measured MRI signal (e.g. physicists) and specialized data analysis methods (i.e. data scientists), eventually. As such, optimization of quantitative MRI - based on a single or multiple MR parameter(s) - is vital to better reflect the underlying tissue properties and dynamics of biological processes, including changes in the homeostasis of mac- 
romolecular compounds due to disease. Here, because of their clinical relevance in, for example, Parkinson's (PD) and Alzheimer's Disease (AD), iron and neuromelanin, have been one of the main targets of such interdisciplinary research (Keren et al., 2015; Bulk et al., 2018). As a result of histological and biochemical validation studies, MRI sequences can be optimized to better image and characterize brain structures in vivo, due to an improved understanding of their biochemical composition (Priovoulos et al., 2017; Ropele and Langkammer, 2017). Obviously, these methods can also be extended towards other clinical phenotypes, including optimized detection of microbleeds and iron depositions in T2DM subjects. Finally, machine-learning methods that target these features, may facilitate diagnosis and prognosis by a clinician.

\section{5. | MULTI-PARAMETER, QUANTITATIVE MRI-BASED CORTICAL PARCELLATION}

Despite the many advantages of acquiring high-resolution data, continuous developments and prospects for clinical use, its high dimensionality and resulting low statistical power (especially in whole-brain studies), necessitates the improvement of data analysis methods as well. As discussed in Chapter 5, the brain phenotype dimensionality can be reduced using parcellation techniques. By this means, data complexity is decreased while statistical power is increased. Automatic segmentation software packages, such as FreeSurfer, typically use macro-anatomical (gyral and sulcal) landmarks and/or predefined atlases to subdivide the (sub-)cortical GM into distinct regions (Fischl et al., 2004; Desikan et al., 2006). More and more, the focus of MRI-data based cortical parcellation is shifting towards individualized methods based on in vivo morphological (i.e. cortical thickness), microstructural (i.e. $\mathrm{T}_{1}$ and $\mathrm{T}_{2}{ }^{*}$ ) or anatomical (i.e. DTI) and functional (i.e. task- or rs-fMRI) connectivity derivates (Geyer et al., 2011; Yeo et al., 2011; Ganepola et al., 2017).

One of the most important advances in this field was the release of a cortical parcellation map based on multiple modalities, including cortical thickness, myelin content (i.e. $T_{1} / T_{2}$ ratio) and (task- and resting state-based) functional $3 T$ data from the Human Connectome Project (Glasser et al., 2016). Here, a gradient-based parcellation approach was applied to identify areal borders using predefined criteria. These criteria were (i) consistency across modalities, (ii) left-right symmetry, (iii) imaging artifacts independency, (iv) strong statistical differences of modalities across opposite sides of the border and ( $v$ ) correspondence with published boundary. After identification of the areas on the group-level, machine-learning algorithms were applied to automatically classify/label each region based on the multi-modal fingerprint on an individual basis 


\section{CHAPTER 6}

in a separate dataset. While the classifier nicely replicated the cortical parcellation across datasets, demonstrating its robustness, it was derived from partially manual procedure used by two neuroanatomists to cross-check and eventually to modify automatically determined areal borders.

In Chapter 3, we applied the same method (i.e. computation of gradients) on the $T_{1}$ and $\mathrm{T}_{2}{ }^{*}$ surface maps. Small differences, for example close to the auditory cortex, were noticeable between $T_{1}$ and $T_{2}{ }^{*}$-based boundaries across the cortex. This confirms that the $\mathrm{T}_{1}$ and $\mathrm{T}_{2}{ }^{*}$ contrasts are not redundant and provide complimentary information. However, the borders varied in quality depending on the parameter and were not continuous, therefore demanding observer input when comparing parameters (i.e., a choice of the gradient threshold value and filling-in gaps in the borders). As such, other studies have explored ways to obtain fully-automatic, data-driven cortical parcellations, based on, for example, clustering techniques. Methods to perform such operations at the single-subject or group-based level, using a single modality (i.e. fMRI) instead, have been recently systematically compared (Arslan et al., 2017). They show that, from the tested methods, none of them outperformed the others. Prioritization of the evaluated method characteristics (e.g. reproducibility, cluster validity or other) may guide the method of choice. In addition, other factors to consider are the: (i) single-subject and group-based performance and possibility to include (ii) multiple contrasts and (iii) spatial constraints.

While none of the previous studies in the field of cortical parcellation explored the use of CBF, the 7T data presented in this thesis provides excellent opportunities to explore the benefits of including additional information based on the brain's baseline perfusion (i.e. CBF). Since CBF is closely coupled to brain metabolism (Raichle, 1998), a data-driven parcellation of brain regions that incorporates perfusion information will presumably enhance subsequent functional analysis. Previous studies demonstrated that perfusion could be used to differentiate primary from secondary auditory and visual areas due to a higher vascular density and metabolic demands (Weber et al., 2008; Gardumi et al., 2017). Preliminary analyses using current data showed that the effect of CBF inclusion is different across sets of regions, presumably related to the differences in baseline brain metabolism across the brain (Haast et al., 2017). The CBF data exhibited a spatial pattern different from $T_{1}, T_{2}^{*}$ and cortical thickness, e.g. similar higher perfusion can be clearly observed in the occipital lobe and posterior cingulate cortex, while $T_{1}$ displays differences between these two areas. It should be noted, however, that the CBF data were acquired with a lower spatial resolution, and therefore, has lower spatial specificity. As these results are promising, we are currently 
extending this towards a fully automatic single-subject and/or group-based cortical parcellation routine, using hierarchical clustering that is driven by multiple parameters.

Hierarchical clustering aims to identify voxels or vertices with comparable characteristics based on single or multiple contrasts (Thirion et al., 2014). In addition, spatial constraints can be imposed on the clustering, leading to clusters containing connected voxels or vertices only. As such, clusters are only formed from parents that share neighboring voxels/vertices based on a predefined connectivity matrix. Fig. 6.1 shows an example group-average $(\mathrm{N}=15)$ cortical parcellation $\left(\mathrm{N}_{\text {regions }}=180\right)$ based on surface maps computed from the quantitative $T_{1}, T_{2}{ }^{*}, C B F$ and cortical thickness data presented in Chapter 4 (control subjects). We used the spatially-constrained Ward's hierarchical clustering algorithm implemented in the SciKit-learn toolbox (Ward Jr, 1963; Pedregosa et al., 2011). In particular, Ward's algorithm is very efficient in processing high-dimensional MRI data. This preliminary multi-parameter cortical parcellation, for example, divides the cingulate cortex into separate regions and also reveals the typically shape of the motor cortex.
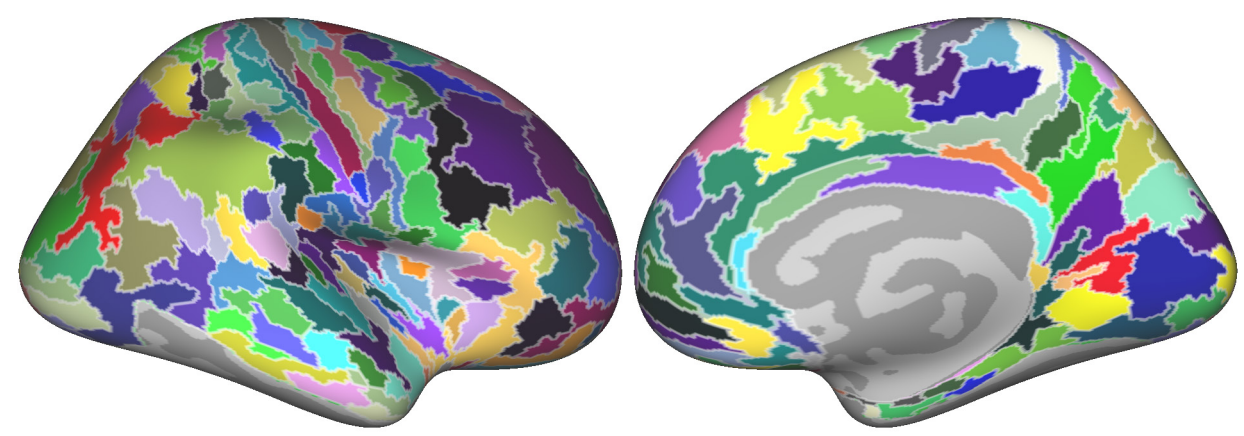

Fig. 6.1. | Multi-parameter cortical parcellation. Quantitative cortical thickness, $T 1, T 2 *$ and CBF data from 15 subjects were used to parcellate the cortical GM into separate regions $\left(N_{\text {regions }}=180\right)$. Please note that the inferior temporal gyrus is masked out as it suffers from strong susceptibility-related artifacts.

However, in contrast to the gradients-based method, hierarchical clustering requires the number of clusters to be pre-defined. In the example above, we selected the same number of regions found in the paper by Glasser et al. (2016). Several methods can be used to find the optimal number of clusters based on the available data. This includes, for example, the Silhouette index, which scores the (data-wise) closeness of each vertex in one cluster to vertices in the neighboring clusters (Rousseeuw, 1987). In addition, bootstrap metrices to evaluate clustering consistency (e.g. adjusted mutual index or adjusted rand index) or cross-validation procedures may be appropriate to find the optimal number of clusters or test the effect of CBF on the cortical parcel- 


\section{CHAPTER 6}

Fig. 6.2. | Evaluation of the Silhouette score for finding the optimal number of clusters using simulated data with varying levels of noise. As can be observed and as expected, the performance of the Silhouette score (i.e. 1 suggests optimal delineation between areas) decreases with increasing noise levels.

lation (Thirion et al., 2014). However, the problem of number of parcels optimization remains open and should be addressed in a data-driven fashion, including simulations. We are currently characterizing, using simulations, the behavior of several methods that aim at identifying the optimal number of clusters. Here, we generate spatial 2D patterns, representing a single parameter, with a known ground truth (i.e. number of clusters) and varying levels of noise. This can be extended to
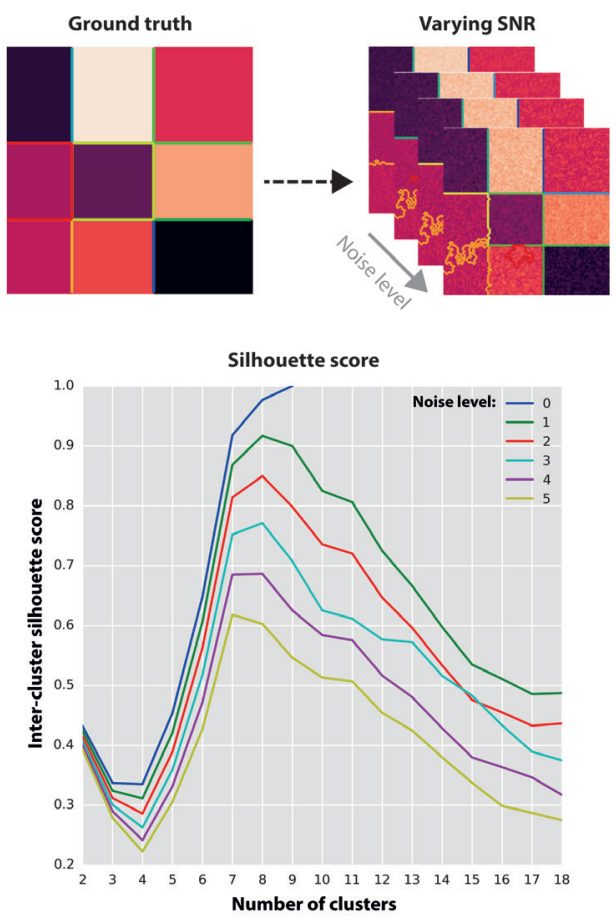
multi-parametric data with corresponding levels of noise (i.e. mimicking the intensity range and quality of cortical thickness, $\mathrm{T}_{1}, \mathrm{~T}_{2}^{*}$ and (BF maps) and to patterns more closely resembling the brain's structure. For example, Fig. 6.2 shows the Silhouette scores for clustering performance across a range of $\mathrm{N}_{\text {clusters }}$ (i.e. 1 suggests optimal cluster separation), based on simulated single parameter data with varying noise and a known ground truth $\left(\mathrm{N}_{\text {clusters }}=9\right)$. Here, the same clustering method was used as for the example in Fig. 6.1, using Ward's criteria and connectivity constraints and shows that performance of the Silhouette score decreases with increasing noise levels. Finally, extending this work towards the larger dataset described in Chapter 5 can address its reproducibility/stability across multiple datasets, as well as identify regions that are most distinct between controls and T2DM or correlate with specific cognitive scores, which are expected to be found mostly in the frontal and temporal lobe.

\section{6. | CONCLUSION}

Taken together, the work in this thesis demonstrated possible applications of a standard quantitative brain 7T MRI protocol. The possibilities of 7T MRI are far-reaching and need continuous developments, ranging from development of hardware to 
analysis software, that enable acquisition of high quality data and processing this data in the most optimal way. The current work extends earlier studies that have shown that 7T MRI is feasible and superior in many instances to 3T MRI and that we are at the doorstep of its routine use in both healthy subjects and diverse clinical populations, eventually combined with other data modalities.

\section{7. | REFERENCES}

Arslan, S., S. I. Ktena, A. Makropoulos, E. C. Robinson, D. Rueckert and S. Parisot (2017). “Human brain mapping: A systematic comparison of parcellation methods for the human cerebral cortex." Neuroimage.

Barmet, C., N. De Zanche and K. P. Pruessmann (2008). "Spatiotemporal magnetic field monitoring for MR." Magn Reson Med 60(1): 187-197.

Bogner, W., M. Chmelik, O. C. Andronesi, A. G. Sorensen, S. Trattnig and S. Gruber (2011). "In vivo $31 \mathrm{P}$ spectroscopy by fully adiabatic extended image selected in vivo spectroscopy: a comparison between 3 T and 7 T." Magn Reson Med 66(4): 923-930.

Brink, W. M., V. Gulani and A. G. Webb (2015). “Clinical applications of dual-channel transmit MRI: A review." J Magn Reson Imaging 42(4): 855-869.

Bulk, M., W. M. Abdelmoula, R. J. A. Nabuurs, L. M. van der Graaf, C. W. H. Mulders, A. A. Mulder, .. . L. van der Weerd (2018). "Postmortem MRI and histology demonstrate differential iron accumulation and cortical myelin organization in early- and late-onset Alzheimer's disease." Neurobiol Aging 62: 231-242.

Cloos, M., N. Boulant, M. Luong, G. Ferrand, E. Giacomini, D. Le Bihan and A. Amadon (2012). "kT-points: short three-dimensional tailored RF pulses for flip-angle homogenization over an extended volume." Magnetic resonance in medicine 67(1): 72-80.

Cohen-Adad, J. (2014). "What can we learn from T2* maps of the cortex?" Neuroimage 93 Pt 2: 189-200.

De Cocker, L. J., A. Lindenholz, J. J. Zwanenburg, A. G. van der Kolk, M. Zwartbol, P. R. Luijten and J. Hendrikse (2016). "Clinical vascular imaging in the brain at 7T." Neuroimage.

Desikan, R. S., F. Segonne, B. Fischl, B. T. Quinn, B. C. Dickerson, D. Blacker, ... R. J. Killiany (2006). "An automated labeling system for subdividing the human cerebral cortex on MRI scans into gyral based regions of interest." Neuroimage 31(3): 968-980.

Feinberg, D. A., A. Beckett and L. Chen (2013). "Arterial spin labeling with simultaneous multislice echo planar imaging." Magn Reson Med 70(6): 1500-1506.

Fischl, B., A. van der Kouwe, C. Destrieux, E. Halgren, F. Segonne, D. H. Salat, ... A. M. Dale (2004). "Automatically parcellating the human cerebral cortex." Cereb Cortex 14(1): 11-22.

Ganepola, T., Z. Nagy, A. Ghosh, T. Papadopoulo, D. C. Alexander and M. I. Sereno (2017). “Using diffusion MRI to discriminate areas of cortical grey matter." Neuroimage.

Gardener, A. G., P. A. Gowland and S. T. Francis (2009). "Implementation of quantitative perfusion imaging using pulsed arterial spin labeling at ultra-high field." Magn Reson Med 61 (4): 874-882.

Gardumi, A., D. Ivanov, M. Havlicek, E. Formisano and K. Uludag (2017). “Tonotopic maps in human auditory cortex using arterial spin labeling." Hum Brain Mapp 38(3): 1140-1154.

Geyer, S., M. Weiss, K. Reimann, G. Lohmann and R. Turner (2011). “Microstructural Parcellation of the Human Cerebral Cortex - From Brodmann's Post-Mortem Map to in vivo Mapping with HighField Magnetic Resonance Imaging." Front Hum Neurosci 5: 19.

Ghariq, E., W. M. Teeuwisse, A. G. Webb and M. J. van Osch (2012). “Feasibility of pseudocontinuous arterial spin labeling at 7 T with whole-brain coverage." MAGMA 25(2): 83-93. 


\section{CHAPTER 6}

Glasser, M. F., T. S. Coalson, E. C. Robinson, C. D. Hacker, J. Harwell, E. Yacoub, . . D. D. Van Essen (2016). "A multi-modal parcellation of human cerebral cortex." Nature 536(7615): 171-178.

Glasser, M. F. and D. C. Van Essen (2011). "Mapping human cortical areas in vivo based on myelin content as revealed by T1- and T2-weighted MRI." J Neurosci 31(32): 11597-11616.

Govindarajan, S. T., J. Cohen-Adad, M. P. Sormani, A. P. Fan, C. Louapre and C. Mainero (2015). "Reproducibility of T2 * mapping in the human cerebral cortex in vivo at 7 tesla MRI." J Magn Reson Imaging 42(2): 290-296.

Haast, R. A., D. Ivanov, E. Formisano and K. Uludag (2017). "Cerebral blood flow as a marker for cortical parcellation." Proc. Intl. Soc. Mag. Reson. Med. 25: 352.

Hurley, A. C., A. Al-Radaideh, L. Bai, U. Aickelin, R. Coxon, P. Glover and P. A. Gowland (2010). “Tailored RF pulse for magnetization inversion at ultrahigh field." Magn Reson Med 63(1): 51-58.

Ivanov, D., A. Gardumi, R. A. M. Haast, J. Pfeuffer, B. A. Poser and K. Uludag (2017). “Comparison of 3T and 7T ASL techniques for concurrent functional perfusion and BOLD studies." Neuroimage 156: 363-376.

Ivanov, D., B. A. Poser, L. Huber, J. Pfeuffer and K. Uludag (2017). “Optimization of simultaneous multislice EPI for concurrent functional perfusion and BOLD signal measurements at 7T." Magn Reson Med 78(1): 121-129.

Katscher, U., P. Börnert, C. Leussler and J. S. Van Den Brink (2003). "Transmit sense." Magnetic resonance in medicine 49(1): 144-150.

Keren, N. I., S. Taheri, E. M. Vazey, P. S. Morgan, A. C. Granholm, G. S. Aston-Jones and M. A. Eckert (2015). "Histologic validation of locus coeruleus MRI contrast in post-mortem tissue." Neuroimage 113: 235-245.

Kim, T., W. Shin, T. Zhao, E. B. Beall, M. J. Lowe and K. T. Bae (2013). “Whole brain perfusion measurements using arterial spin labeling with multiband acquisition." Magn Reson Med 70(6): 1653-1661.

Kircher, M., D. M. Witten, P. Jain, B. J. O'Roak, G. M. Cooper and J. Shendure (2014). "A general framework for estimating the relative pathogenicity of human genetic variants." Nat Genet 46(3): 310-315.

Lutti, A., F. Dick, M. I. Sereno and N. Weiskopf (2014).“Using high-resolution quantitative mapping of R1 as an index of cortical myelination." Neuroimage 93 Pt 2: 176-188.

Maclaren, J., M. Herbst, O. Speck and M. Zaitsev (2013). “Prospective motion correction in brain imaging: a review." Magn Reson Med 69(3): 621-636.

Marques, J. P. and R. Gruetter (2013). "New developments and applications of the MP2RAGE sequence--focusing the contrast and high spatial resolution R1 mapping." PLoS One 8(7): e69294.

Marques, J. P., T. Kober, G. Krueger, W. van der Zwaag, P. F. Van de Moortele and R. Gruetter (2010). "MP2RAGE, a self bias-field corrected sequence for improved segmentation and T1-mapping at high field." Neuroimage 49(2): 1271-1281.

Marques, J. P. and D. G. Norris (2017). “How to choose the right MR sequence for your research question at 7T and above?" Neuroimage.

Mougin, O., R. Abdel-Fahim, R. Dineen, A. Pitiot, N. Evangelou and P. Gowland (2016). "Imaging gray matter with concomitant null point imaging from the phase sensitive inversion recovery sequence." Magn Reson Med 76(5): 1512-1516.

Oliver-Taylor, A., R. Ordidge, C. Randell and D. L. Thomas (2012). “Parallel Transmit Vessel Selective Arterial Spin Labelling: Phantom and In-Vivo Results." Proc. Intl. Soc. Mag. Reson. Med. 20: 1995.

Pedregosa, F., G. Varoquaux, A. Gramfort, V. Michel, B. Thirion, O. Grisel, . . V. Dubourg (2011). "Scikit-learn: Machine learning in Python." Journal of machine learning research 12(Oct): 28252830.

Pohmann, R., O. Speck and K. Scheffler (2016). "Signal-to-noise ratio and MR tissue parameters in human brain imaging at 3, 7, and 9.4 tesla using current receive coil arrays." Magn Reson Med 
75(2): 801-809.

Polimeni, J. R. and K. Uludağ (2018). "Neuroimaging with Ultra-High Field MRI: Present and Future." Neurolmage.

Poser, B. A., R. J. Anderson, B. Guérin, K. Setsompop, W. Deng, A. Mareyam, ... V. A. Stenger (2014). "Simultaneous multislice excitation by parallel transmission." Magnetic resonance in medicine 71(4): 1416-1427.

Priovoulos, N., H. I. L. Jacobs, D. Ivanov, K. Uludag, F. R. J. Verhey and B. A. Poser (2017). “High-resolution in vivo imaging of human locus coeruleus by magnetization transfer MRI at 3T and 7T." Neuroimage.

Raichle, M. E. (1998). "Behind the scenes of functional brain imaging: a historical and physiological perspective." Proc Natl Acad Sci U S A 95(3): 765-772.

Ropele, S. and C. Langkammer (2017). "Iron quantification with susceptibility." NMR Biomed 30(4).

Rousseeuw, P. J. (1987). "Silhouettes: a graphical aid to the interpretation and validation of cluster analysis." Journal of computational and applied mathematics 20: 53-65.

Saeys, Y., I. Inza and P. Larranaga (2007). "A review of feature selection techniques in bioinformatics." Bioinformatics 23(19): 2507-2517.

Sengupta, S., A. Roebroeck, V. G. Kemper, B. A. Poser, J. Zimmermann, R. Goebel and G. Adriany (2016). "A specialized multi-transmit head coil for high resolution fMRI of the human visual cortex at 7T." PloS one 11(12): e0165418.

Setsompop, K., V. Alagappan, B. Gagoski, T. Witzel, J. Polimeni, A. Potthast, ... L. L. Wald (2008). "Slice-selective RF pulses for in vivo B 1+ inhomogeneity mitigation at 7 tesla using parallel RF excitation with a 16-element coil." Magnetic resonance in medicine 60(6): 1422-1432.

Shajan, G., M. Kozlov, J. Hoffmann, R. Turner, K. Scheffler and R. Pohmann (2014). “A 16-channel dual-row transmit array in combination with a 31-element receive array for human brain imaging at 9.4 T." Magnetic resonance in medicine 71(2): 870-879.

Stuber, C., M. Morawski, A. Schafer, C. Labadie, M. Wahnert, C. Leuze, ... R. Turner (2014). "Myelin and iron concentration in the human brain: a quantitative study of MRI contrast." Neuroimage 93 Pt 1: 95-106.

Teeuwisse, W. M., W. M. Brink and A. G. Webb (2012). "Quantitative assessment of the effects of high-permittivity pads in 7 Tesla MRI of the brain." Magn Reson Med 67(5): 1285-1293.

Teeuwisse, W. M., A. G. Webb and M. J. van Osch (2010). "Arterial spin labeling at ultra-high field: All that glitters is not gold." International Journal of Imaging Systems and Technology 20(1): 62-70.

Thirion, B., G. Varoquaux, E. Dohmatob and J. B. Poline (2014). “Which fMRI clustering gives good brain parcellations?" Front Neurosci 8: 167.

Trattnig, S., E. Springer, W. Bogner, G. Hangel, B. Strasser, B. Dymerska, .. S. D. Robinson (2016). "Key clinical benefits of neuroimaging at 7T." Neuroimage.

Ugurbil, K. (2017). “Imaging at ultrahigh magnetic fields: History, challenges, and solutions.” roimage.

Uludag, K. and P. Blinder (2017). “Linking brain vascular physiology to hemodynamic response in ultra-high field MRI." Neuroimage.

van Gelderen, P., J. A. de Zwart, J. Lee, P. Sati, D. S. Reich and J. H. Duyn (2012). “Nonexponential $\mathrm{T}(2)$ decay in white matter." Magn Reson Med 67(1): 110-117.

Voelker, M. N., O. Kraff, D. Brenner, A. Wollrab, O. Weinberger, M. C. Berger, ... O. Speck (2016). "The traveling heads: multicenter brain imaging at 7 Tesla." MAGMA 29(3): 399-415.

Vu, A. T., E. Auerbach, C. Lenglet, S. Moeller, S. N. Sotiropoulos, S. Jbabdi, ... K. Ugurbil (2015). "High resolution whole brain diffusion imaging at 7T for the Human Connectome Project." $\mathrm{Neu}$ roimage 122: 318-331. 


\section{CHAPTER 6}

Ward Jr, J. H. (1963). "Hierarchical grouping to optimize an objective function." Journal of the American statistical association 58(301): 236-244.

Weber, B., A. L. Keller, J. Reichold and N. K. Logothetis (2008). “The microvascular system of the striate and extrastriate visual cortex of the macaque." Cereb Cortex 18(10): 2318-2330.

Weiskopf, N., J. Suckling, G. Williams, M. M. Correia, B. Inkster, R. Tait, .. . A. Lutti (2013). “Quantitative multi-parameter mapping of R1, $\mathrm{PD}\left({ }^{*}\right), \mathrm{MT}$, and $\mathrm{R} 2\left(^{*}\right)$ at $3 \mathrm{~T}$ : a multi-center validation." Front Neurosci 7: 95.

Wyss, M., Y. Duerst, D. Nanz, L. Kasper, B. J. Wilm, B. E. Dietrich, .. K. P. Pruessmann (2017). "Feedback field control improves the precision of T2 * quantification at 7T." NMR Biomed 30(10).

Xu, F., W. Li, P. Liu, J. Hua, J. J. Strouse, J. J. Pekar, ... Q. Qin (2018). “Accounting for the role of hematocrit in between-subject variations of MRI-derived baseline cerebral hemodynamic parameters and functional BOLD responses." Hum Brain Mapp 39(1): 344-353.

Yeo, B. T., F. M. Krienen, J. Sepulcre, M. R. Sabuncu, D. Lashkari, M. Hollinshead, . . R. L. Buckner (2011). "The organization of the human cerebral cortex estimated by intrinsic functional connectivity." J Neurophysiol 106(3): 1125-1165.

Zhu, Y. (2004). "Parallel excitation with an array of transmit coils." Magnetic Resonance in Medicine 51(4): 775-784. 
ADDENDA 


\subsection{KNOWLEDGE VALORIZATION}

Besides scientific merit, the experiments and their outcomes, presented in this thesis, also carry social and economic value and implications. In the following, we place the research approach and results into a broader perspective and indicate the valorization potential of the main findings.

Following the title of this thesis ("Quantitative 7T MRI in healthy subjects and in metabolic diseases"), the main findings can be approached from two perspectives, either from (i) the 7T MRI user's (Chapters 2 and 3) or (ii) a clinician's (Chapters 4 and 5) point of view. As such, in the following, we will discuss the socioeconomic benefits of the current work for each of them.

\subsection{1. | Ultra-high field MRI user}

MRI has become one of the major clinical diagnostic tools used worldwide. It allows non-invasive imaging and characterization of the human body, based on the principles described in Chapter 1, and thus, enables detection of changes and abnormalities due to development, aging or disease. Since its initial discovery (Lauterbur, 1973; Mansfield and Grannell, 1973), there has been tremendous developments related to almost every aspect of $\mathrm{MRI}$, ranging from better hardware to optimized analysis strategies. Here, 7T MRI has been one of the most significant advances (Ugurbil, 2017). Although the technology has been in use for over 15 years with > 70 7T scanners worldwide (see Fig. 7.1), the recent FDA and CE approval for the Terra system, manufactured by Siemens Healthineers, will make 7T MRI better accessible for non-MRI experts and pushing it towards a wider range of applications.

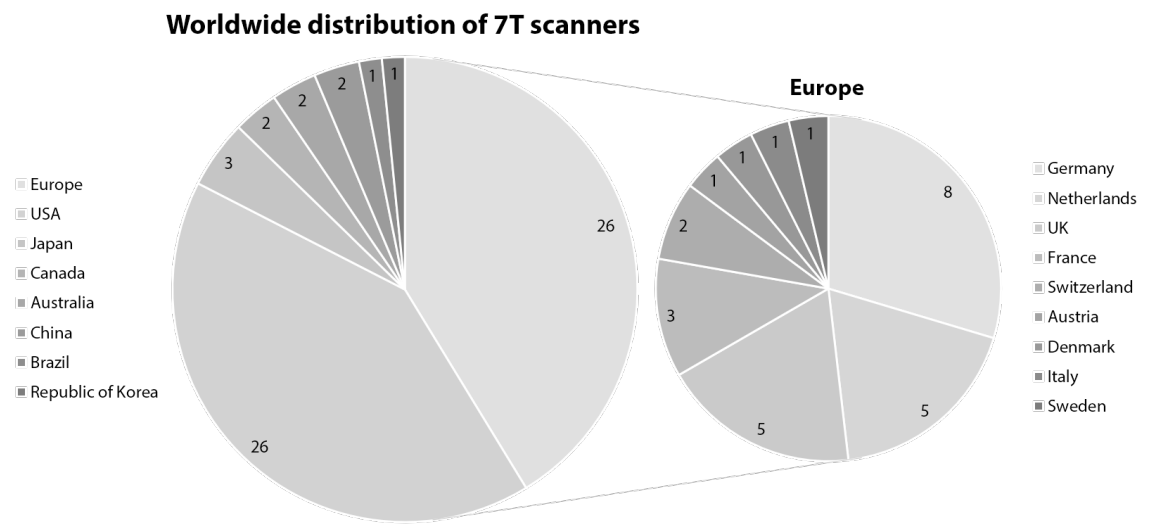

Fig. 7.1. | Overview of worldwide 7TMRI scanners. Please, note that this may not represent the actual status due to contineous installation of new machines. 
While progress has plateaued at conventional field strengths (i.e. $\leq 3 \mathrm{~T}$ ), the increasingly availability of 7T scanners enables to continuate the growing potential of MRI as a diagnostic tool in, for example, neurodegenerative diseases. This is mainly driven by the higher field strength, leading to increased data quality (i.e. signal-to-noise) (Pohmann et al., 2016), and higher spatial and temporal imaging resolutions (Pfeuffer et al., 2002; Yacoub et al., 2008). As such, subtle changes in brain anatomy and vasculature have shown to be better detectable compared to standard clinical MRI (i.e. due to improved contrast-to-noise) (Trattnig et al., 2016).

However, clinical decision-making, as well as studies on disease-, aging- and/or development-related changes in the brain, using 7T MRI, requires reproducible (i.e. precise) and reliable data. The importance of this, mainly with respect to across studies, sites and manufacturer generalizability of (ultra-high field) MRI data, has been discussed previously in the general discussion. However, as summarized in the previous chapter, the results in Chapters 2 and 3 show the potential and relative performance of some of the commonly used quantitative anatomical (i.e. $\mathrm{T}_{1}$ and $\mathrm{T}_{2}{ }^{*}$ ) imaging approaches. These were evaluated, mostly with respect to in vivo myelin mapping, in terms of reproducibility (i.e. coefficient of variation) and quality (i.e. contrast-to-nose), as well as the effect of magnetic field (i.e. $\mathrm{B}_{1}^{+}$) inhomogeneities on morphological measurements (i.e. cortical thickness). Especially the latter is of great importance for users working in a clinical setting and requires accurate measurements. As such, the work and results of the current thesis contributes to a better understanding of the relative performance of (quantitative) $\mathrm{T}_{1}$ and $\mathrm{T}_{2}{ }^{*}$ imaging methods at $7 \mathrm{~T}$. It shows how they could be reliable acquired and analyzed by others that are interested in the use of these sequences, such as in patient populations, or in healthy subjects for similar applications (e.g. cortical parcellation and/or myelin mapping). Eventually and from a more business-type of perspective, standardized protocols, validated across different imaging sites and scanner vendors, as well as the workflows to analyze the acquired data, can be used as service products by MR imaging companies to improve accessibility of 7T MRI.

\subsection{2. | Clinical perspective}

While Chapters 2 and 3 are mainly useful for (future) 7T MRI users, the experiments in Chapters 4 and 5 are especially important for clinicians involved in the treatment of individuals affected by the m.3243A $>$ G mutation or with Type 2 Diabetes Mellitus (T2DM), respectively. The impact of the current research on each of them will be discussed in the following paragraphs. 
The m.3243A>G mutation is one of the most common mitochondrial mutations with a carrier rate of 1 in 400 people (Elliott et al., 2008). It can induce a wide-range of clinical symptoms leading to a diverse phenotypic spectrum (Nesbitt et al., 2013). Better knowledge of the genotype (e.g. mutation load) - phenotype (e.g. brain) relationship may potentially enhance patient prognosis. We showed that the m.3243A $>$ G mutation load (i.e. percentage of affected mitochondria in a single cell) in blood (as well as urine) correlated significantly with the brain phenotype: the patients with a higher mutation load were characterized by a lower brain volume. Especially cortical gray matter thickness, but also $\mathrm{T}_{1}, \mathrm{~T}_{2}{ }^{*}$ and $\mathrm{CBF}$, were affected. This was predominantly restricted to (e.g. auditory or frontal lobe) regions that can be linked to the observed symptoms (e.g. impaired hearing) and/or cognitive performance (e.g. Stroop task). Although mutation load may not be the only contributor to disease severity, it may provide an initial scale for clinical geneticists and neurologists, working with $\mathrm{m} .3243 \mathrm{~A}>\mathrm{G}$ patients, to estimate the patient's prognosis, in terms of the brain phenotype and cognitive performance. In addition, these results can guide future pathobiological studies towards more targeted experiments to disentangle the underlying biochemical changes. Alternatively, it may facilitate testing the effect of possible intervention strategies, such as arginine supplementation, on the progression of brain atrophy (El-Hattab et al., 2012). Eventually, improved follow-up and management of patients harboring the m.3243A $>G$, may improve their quality-of-life. Finally, while both the m.3243A>G and T2DM affect specific metabolic pathways, T2DM is much more prevalent and therefore carries a higher socioeconomic burden. As a result, much more studies focus on T2DM-related pathologies and little work is done to systematically characterize the neurodegenerative changes induced by the m.3243A>G genotype. Therefore, the current work may have significant impact on the respective field due to the relatively large number of m.3243A > G patients that was recruited.

T2DM is a metabolic disease and, in contrast to patients affected by the m.3243A $>\mathrm{G}$ genotype, characterized by high global prevalence. The total number of people with T2DM was estimated at 171 million individuals world-wide in 2000 and is projected to rise to 366 million in 2030 (Wild et al., 2004). Patients with T2DM have an increased risk to develop dementia (Biessels et al., 2006), which may eventually result in significant impairment of daily life and quality-of-life. Early identification of (pre-)diabetic subjects at risk for cognitive decline, as a result of neurodegenerative changes, is vital to keep the economic and emotional burden at a minimum level. In the current thesis, we have combined genetic, metabolomic and MR imaging with extensive phenotypical (e.g. clinical and cognitive) data to put together a unique dataset. This dataset allows extensive phenotyping by genetics, metabolomics, MRI, but also "systems biology" ex- 
perts to highlight features that differ between controls without diabetes, pre-diabetic and diabetic subjects, or find novel subgroups. Eventually, identification of biomarkers that are able to characterize the subjects with an increased risk of cognitive decline and/or dementia will improve diabetes care, for example, through personalized medicine, next to standard diabetes treatment strategies, and significantly impact the socioeconomic burden of T2DM. At the same time, this will also provide possibilities for the pharmaceutical industry to improve the impact of their products due to more effective treatment.

\subsection{3. | References}

Biessels, G. J., S. Staekenborg, E. Brunner, C. Brayne and P. Scheltens (2006). "Risk of dementia in diabetes mellitus: a systematic review." Lancet Neurol 5(1): 64-74.

El-Hattab, A. W., J. W. Hsu, L. T. Emrick, L. J. Wong, W. J. Craigen, F. Jahoor and F. Scaglia (2012). "Restoration of impaired nitric oxide production in MELAS syndrome with citrulline and arginine supplementation." Mol Genet Metab 105(4): 607-614.

Elliott, H. R., D. C. Samuels, J. A. Eden, C. L. Relton and P. F. Chinnery (2008). “Pathogenic mitochondrial DNA mutations are common in the general population." Am J Hum Genet 83(2): 254-260.

Lauterbur, P. (1973). “Image formation by induced local interactions: examples employing nuclear magnetic resonance."

Mansfield, P. and P. K. Grannell (1973). “NMR'diffraction'in solids?" Journal of Physics C: solid state physics 6(22): L422.

Nesbitt, V., R. D. Pitceathly, D. M. Turnbull, R. W. Taylor, M. G. Sweeney, E. E. Mudanohwo, .. R. McFarland (2013). "The UK MRC Mitochondrial Disease Patient Cohort Study: clinical phenotypes associated with the m.3243A $>\mathrm{G}$ mutation--implications for diagnosis and management." J Neurol Neurosurg Psychiatry 84(8): 936-938.

Pfeuffer, J., P. F. van de Moortele, E. Yacoub, A. Shmuel, G. Adriany, P. Andersen, ... X. Hu (2002). "Zoomed functional imaging in the human brain at 7 Tesla with simultaneous high spatial and high temporal resolution." Neuroimage 17(1): 272-286.

Pohmann, R., O. Speck and K. Scheffler (2016). "Signal-to-noise ratio and MR tissue parameters in human brain imaging at 3, 7, and 9.4 tesla using current receive coil arrays." Magn Reson Med 75(2): 801-809.

Trattnig, S., E. Springer, W. Bogner, G. Hangel, B. Strasser, B. Dymerska, . . S. D. Robinson (2016). "Key clinical benefits of neuroimaging at 7T." Neuroimage.

Ugurbil, K. (2017).“"Imaging at ultrahigh magnetic fields: History, challenges, and solutions.” Neuroimage.

Wild, S., G. Roglic, A. Green, R. Sicree and H. King (2004). "Global prevalence of diabetes: estimates for the year 2000 and projections for 2030." Diabetes Care 27(5): 1047-1053.

Yacoub, E., N. Harel and K. Ugurbil (2008). "High-field fMRI unveils orientation columns in humans." Proc Natl Acad Sci U S A 105(30): 10607-10612. 


\section{2. | ACKNOWLEDGMENTS}

It is obvious that the contributions to this thesis can not solely be ascribed to the efforts of just one person. Therefore, I would like to express my gratitude to several people that made this possible. First of all, I am thankful for my supervisors, Kâmil, Elia and Jaap, for entrusting me this $\mathrm{PhD}$ project and maintaining this support throughout. Kâmil, you are able to fascinate me, due to your widespread knowledge, as well as your abilities to provide thorough supervision while giving me the sense of freedom. This, topped up with your specific sense of humor, is irreplaceable. For this, I am thankful and I am looking forward to extent this to Toronto. Elia, while you took care of the rest, I was able to focus on the important things: doing science. I really enjoyed our conversions either at work, or when we were fighting the rain on our way to the STW meetings. Jaap, thank you for your valuable and down-to-earth feedback on my progress throughout the years. In addition, I would like to thank Dimo for his endless support along the way. Thanks to your efforts I was able to acquaint with the seemingly complexity of MRI acquisitions and to make it look less complex. I truly hope we will have many opportunities to catch up and keep working together in the future.

Of course, there were many more people involved that made my time in Maastricht easier and much more fun, both professionally as non-professionally. First, without the collaboration of many people, much of the presented would have been impossible. Therefore, René, Rutger (Erasmus MC), Bert, Florence, Bieke, Patrick (Genetics), Michiel (MaCSBio) and Carla (De Maastricht Studie): thank you, I really enjoyed working together. Second, I want to acknowledge the efforts of Christl, Riny, Eva and José, that significantly eased my stay at CN by taking care of bureaucratic and logistic matters. Third, I highly value the time spent with my fellow colleagues, not directly involved in my research. Big thanks for being such a great company and accepting my sports-dominated contributions to our chats during lunch and other social activities. Although my choice of coffee was often a reason for jokes, I always felt comfortable talking to each of you about anything that kept me busy.

Lastly, I want to specifically state my thankfulness to my friends outside of work. In particular, Maarten and Koen. Each minute spent in the gym, at parties, sitting on the couch or around the table consuming enormous amounts of food (and sometimes beers) while having non-sense conversations with you were very valuable. Things as these really enable me to leave work at work. Finally, and most importantly, I want to thank my parents, sister and Anita for their support and for providing me the precious feeling of happiness in life. 


\section{3. | CURRICULUM VITAE}

Roy was born on June $3^{\text {rd }}$, 1989, in Roosendaal, Netherlands. After finishing his secondary education (HAVO) at the Norbertuscollege (Roosendaal, Netherlands) in 2006, he studied Applied Biomedical Life Sciences at Avans Hogeschool (Breda, Netherlands). During his Bachelor internships at University of Sydney (Sydney, New South Wales, Australia), under supervision Dr. Catherine Leamey, and at Radboud University (Nijmegen, Netherlands), under supervision of Prof. Dr. Gerard Martens, he studied the neuronal mechanisms underlying brain plasticity in mice and rats, respectively. He obtained his Bachelor degree in 2010 and continued a Master's program in Medical Biology (Neuroscience track) at Radboud University (Nijmegen, Netherlands). During his first internship at the department of Anatomy he performed behavioral and MRI experiments, under supervision of Dr. Amanda Kiliaan, to study the effects of a multi-nutrient fish oil diet on cognition and pathological features of Alzheimer's disease. His second internship involved characterization of carotid artery pathology using ultrasound measurements in patients treated for head and neck cancer. He completed his Masters in 2013, after which he started a PhD project under the supervision of Prof. Dr. Elia Formisano and Dr. Kâmil Uludağ of Maastricht University. After finishing his thesis entitled "Quantitative brain MRI at 7T in healthy subjects and metabolic diseases" Roy will continue this work in Toronto, Ontario, Canada with Dr. Kâmil Uludağ. 


\section{4. | PUBLICATION LIST}

Peer-reviewed journal articles

Gulban OF, Schneider M, Marquardt I, Haast RAM, De Martino F. A scalable method to improve gray matter segmentation at ultra high field MRI. PLoS One (in press).

Haast RAM, Ivanov D, Uludağ $K$. The impact of $B_{1}{ }^{+}$correction on MP2RAGE cortical $T_{1}$ and apparent cortical thickness at 7T. Hum Brain Mapp. 2018 Feb 18.

Haast RAM, Ivanov D, IJsselstein RJT, Sallevelt SCEH, Jansen JFA, Smeets HJM, de Coo IFM, Formisano E, Uludağ K. Anatomic \& metabolic brain markers of the m.3243A>G mutation: A multi-parametric 7T MRI study. Neuroimage Clin. 2018 Jan 31.

Ivanov D*, Gardumi A*, Haast RAM, Pfeuffer J, Poser BA, Uludağ K. Comparison of 3T and 7T ASL techniques for concurrent functional perfusion and BOLD studies. Neuroimage. 2017 Aug 1;156:363-376 (*authors contributed equally).

Haast RAM, Ivanov D, Formisano E, Uludağ K. Reproducibility and Reliability of Quantitative and Weighted $\mathrm{T}_{1}$ and $\mathrm{T}_{2}{ }^{*}$ Mapping for Myelin-Based Cortical Parcellation at 7 Tesla. Front Neuroanat. 2016 Nov 18;10:112.

Wiesmann M, Zerbi V, Jansen D, Haast RAM, Lütjohann D, Broersen LM, Heerschap A, Kiliaan AJ. A Dietary Treatment Improves Cerebral Blood Flow and Brain Connectivity in Aging apoE4 Mice. Neural Plast. 2016;2016:6846721.

Haast RAM, Kiliaan AJ. Impact of fatty acids on brain circulation, structure and function. Prostaglandins Leukot Essent Fatty Acids. 2015 Jan;92:3-14.

Wilbers J, Dorresteijn LD, Haast RAM, Hoebers FJ, Kaanders JH, Boogerd W, van Werkhoven ED, Nowee ME, Hansen HH, de Korte CL, Kappelle AC, van Dijk EJ. Progression of carotid intima media thickness after radiotherapy: a long-term prospective cohort study. Radiother Oncol. 2014 Dec;113(3):359-63.

Wilbers J, Kappelle AC, Kessels RP, Steens SC, Meijer FJ, Kaanders JH, Haast RAM, Versteeg LE, Tuladhar AM, de Korte CL, Hansen HH, Hoebers FJ, Boogerd W, van Werkhoven ED, Nowee ME, Hart G, Bartelink H, Dorresteijn LD, van Dijk EJ. Long term cerebral and vascular complications after irradiation of the neck in head and neck cancer patients: a prospective cohort study: study rationale and protocol. BMC Neurol. 2014 Jun 19;14:132.

Haast RAM, Gustafson DR, Kiliaan AJ. Sex differences in stroke. $\underline{\text { J Cereb Blood Flow }}$ Metab. 2012 Dec;32(12):2100-7.

Vallès A, Boender AJ, Gijsbers S, Haast RAM, Martens GJ, de Weerd P. Genomewide analysis of rat barrel cortex reveals time- and layer-specific mRNA expression changes related to experience-dependent plasticity. J Neurosci. $2011 \mathrm{Apr}$ 20;31(16):6140-58.

Kneppers AEM, Haast RAM, Langen RCJ, Verdijk LB, Leermakers PA, Gosker H, van Loon LJ, Lainscak M, Schols AMWJ. Unbiased analysis of skeletal muscle molecular responses upon pulmonary rehabilitation in advanced COPD (under review). 


\section{Conference contributions}

Kneppers AEM, Haast RAM, Langen RCJ, Verdijk LB, Leermakers PA, Gosker H, van Loon LJ, Lainscak M, Schols AMWJ. Unbiased analysis of skeletal muscle molecular responses upon pulmonary rehabilitation in advanced COPD. Submitted to annual meeting of European Respiratory Society, Paris, France (2018).

Ivanov D, Kashyap S, Haast RAM, Janssens SEW, Huber L, Poser BA, Uludağ K. Human Whole-brain sub-millimeter cerebral blood flow map using 7T ASL. Submitted to annual meeting of International Society of Magnetic Resonance in Medicine (ISMRM), Paris, France (2018).

Haast RAM, Ivanov D, Formisano E, Uludağ K. Cerebral blood flow as a marker for cortical parcellation. E-poster session presented at annual meeting of ISMRM, Honolulu, HI, USA (2017).

Haast RAM, Ivanov D, Formisano $E$, Uludağ K. The Effects of $B_{1}{ }^{+}$Correction of MP2RAGE on Estimating Cortical Thickness and $\mathrm{T}$ at 7T. E-poster session presented at annual meeting of ISMRM, Honolulu, HI, USA (2017).

Haast RAM, Ivanov D, Jansen JFA, Smeets HJ, de Coo IFM, Formisano E, Uludağ K. Cortical Thickness in Relation to m.3243A>G Mutation Load in MELAS Syndrome. Poster session presented at annual meeting of ISMRM, Honolulu, HI, USA (2017).

Gardumi A, Ivanov D, Haast RAM, Pfeuffer J, Poser BA, Uludağ K. The Influence of Acquisition Parameters on CBF and BOLD Sensitivity in 3 and 7 Tesla ASL FMRI. E-poster session presented at annual meeting of ISMRM, Honolulu, HI, USA (2017).

Haast RAM, Ivanov D, Formisano E, Uludağ K. Multi-Parameter Quantitative Brain Anatomy at 7 Tesla. Oral session presented at annual meeting of Organization of Human Brain Mapping, Geneva, Switzerland (2016).

Haast RAM, Ivanov D, Jansen JFA, Smeets HJ, de Coo IFM, Formisano E, Uludağ K. Quantitative brain imaging in MELAS patients. Oral session presented at ISMRM Workshop on Ultra High Field MRI: Technological Advances \& Clinical Applications, Heidelberg, Germany (2016).

Haast RAM, Ivanov D, Formisano E, Uludağ K. Comparing different contrasts for myelin-related cortical mapping at 7 Tesla. Oral session presented at annual Benelux chapter of ISMRM, Ghent, Belgium (2015) and e-poster session presented at annual meeting of ISMRM, Toronto, Ontario, Canada (2015). 